Portland State University

PDXScholar

Summer 7-18-2018

\title{
Assessment of Seismic Retrofit Prioritization Methodology for Oregon's Highway Bridges Based on the Vulnerability of Highway Segments
}

Selamawit Tesfayesus Mehary

Portland State University

Follow this and additional works at: https://pdxscholar.library.pdx.edu/open_access_etds

Part of the Civil and Environmental Engineering Commons Let us know how access to this document benefits you.

\section{Recommended Citation}

Mehary, Selamawit Tesfayesus, "Assessment of Seismic Retrofit Prioritization Methodology for Oregon's Highway Bridges Based on the Vulnerability of Highway Segments" (2018). Dissertations and Theses. Paper 4509.

https://doi.org/10.15760/etd.6393

This Dissertation is brought to you for free and open access. It has been accepted for inclusion in Dissertations and Theses by an authorized administrator of PDXScholar. Please contact us if we can make this document more accessible: pdxscholar@pdx.edu. 
Assessment of Seismic Retrofit Prioritization Methodology for Oregon's Highway

Bridges Based on the Vulnerability of Highway Segments

by

Selamawit Tesfayesus Mehary

A dissertation submitted in partial fulfillment of the requirements for the degree of

Doctor of Philosophy

in

Civil and Environmental Engineering

\author{
Dissertation Committee: \\ Peter Dusicka, Chair \\ Franz Rad \\ Thomas Schumacher \\ Jiunn-Der (Geoffrey) Duh
}

Portland State University

2018 


\section{ABSTRACT}

Geologists have indicated that the question is not if a catastrophic earthquake will occur in Oregon but when one will occur. Scientists estimate that there is close to 40 percent conditional probability that a Cascadia subduction zone earthquake of magnitude 8.0 or above will strike Oregon in the next 50 years. In addition, the majority of Oregon's bridge inventory was built prior to the current understanding of bridge response and prior to current understanding of the expected earthquake demands. In order to minimize potential bridge damage in the case of an earthquake, one approach is to retrofit seismically deficient bridges. However, often times the decision maker is faced with the difficulty of selecting only a few bridges within the inadequate ones. Hence, the issue of prioritizing upgrading naturally arises. The goal of this study is to assess and refine bridge prioritization methodology to be utilized for ranking Oregon's bridge inventory. CFRP retrofit has been experimentally and analytically evaluated to demonstrate the effectiveness of the technique and was found to be an efficient and economical option. A vulnerability assessment estimates that close to 30 percent of Oregon's highway bridge inventory will sustain moderate damage to collapse. However, retrofitting two most common bridge types in the inventory will reduce the number of damaged bridges by about 70 percent. A cost-benefit assessment that takes into consideration direct and indirect costs associated with damaged bridges and retrofitting of bridges shows that the benefit is up to three times the cost to retrofit. The same principle was applied to rank twelve highway segments for seismic retrofit considered important by Oregon Department of Transportation. One selected segment was considered to be retrofitted and 
vulnerability assessed. The benefit to cost ratios for each assessment was compared and the highway segments were ranked accordingly. The top five segments in the ranking happen to be located in the East-West corridor connecting I-5 to US- 101. 
I would like to dedicate this work to my family. 


\section{ACKNOWLEDGEMENTS}

I would like to express my deepest appreciation to my advisor, Dr. Peter Dusicka, for his unwavering guidance and support. He has provided me with such a wonderful and rewarding research experience.

I would also like to thank my dissertation committee Dr. Franz Rad, Dr. Thomas Schumacher and Dr. Geoffrey Duh for their encouragement and guidance throughout this process and for their willingness to help me through my graduate studies.

My graduate experience would have been incomplete without my fellow students at iSTAR lab. I'm extremely grateful to Dr. Arlindo Lopes, Dr. Ramiro Bazaez, Ilya Palnikov, Joanne Shields, Stuart Garth, Marshall Stokes, Bruno DeToledo, and Tom Bennet. All the long technical and nontechnical discussions have been crucial. In addition, without their help, my experiments would have been close to impossible.

Great thanks also goes to all the staff and faculty in the civil engineering department. A special thank you to Dr. Christopher Monsere, Megan Falcone, Ariel Lewis, and Dr. Annette Dietz for always having their doors open and being available to help with everything they can.

I want to also acknowledge Oregon Department of Transportation (ODOT) and Oregon Transportation Research and Education Consortium (OTREC), for the provided financial assistance.

I would be remiss if I didn't acknowledge the contribution and support I have received from my parents and brothers. Over the years they have expressed confidence in 
my abilities and let me believe that I can accomplish anything I set my mind to. My deep love and appreciation is extended to them.

Finally, I would like to express by deepest and most heartfelt gratitude to my husband, Medhanie, and my children, Gabriel and Azalia. Without their love, encouragement, patience, and selfless sacrifices, this project would not have been possible.

Thank you. 


\section{TABLE OF CONTENTS}

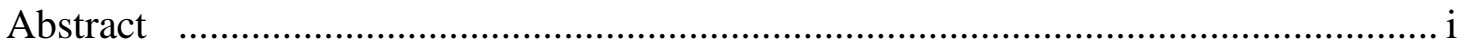

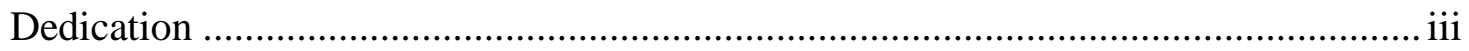

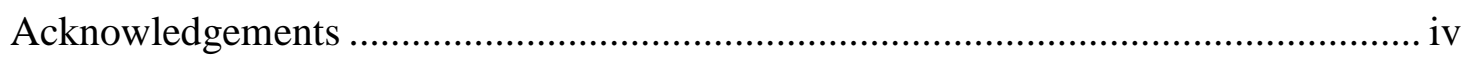

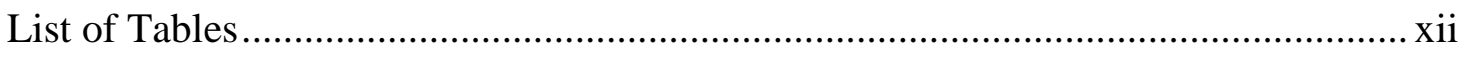

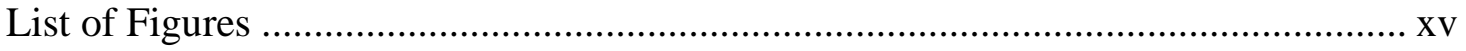

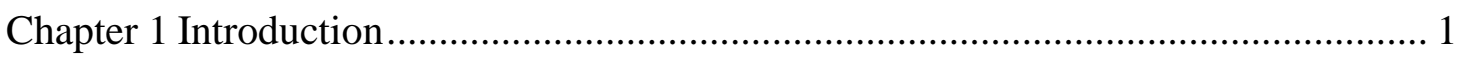

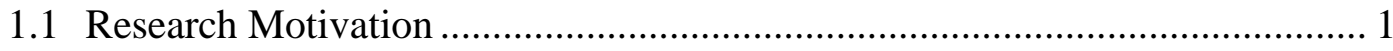

1.2 Objectives and Scope of Research ........................................................ 1

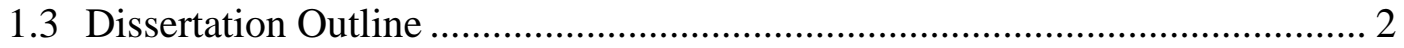

Chapter 2 Vulnerability and Importance Assessment ……………………………..... 5

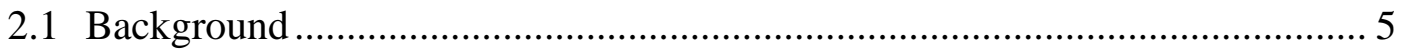

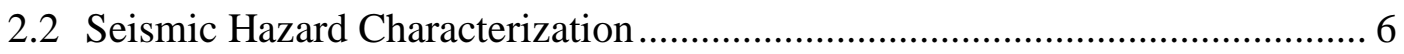

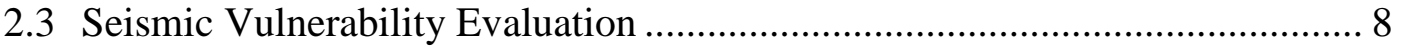

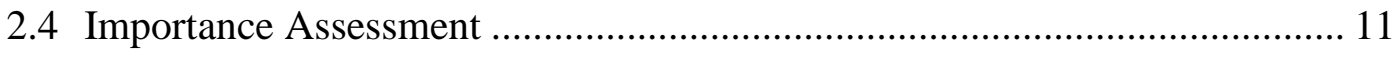

2.5 Seismic Risk Assessment...................................................................... 15

2.5.1 Previous Research on Seismic Vulnerability Assessments .......... 16

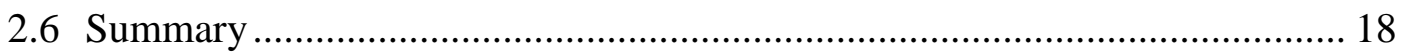

Chapter 3 Seismic Vulnerability Assessments of Oregon's Bridges ........................... 19

3.1 Damage Functions ……………………………........................................ 19

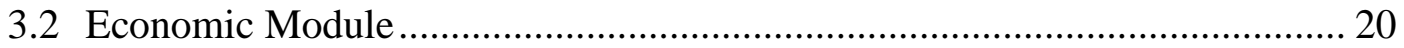

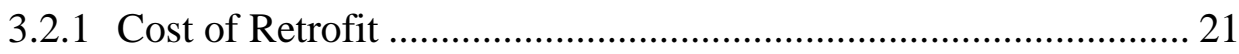

3.2.2 Repair and Replacement Cost ...................................................... 21 


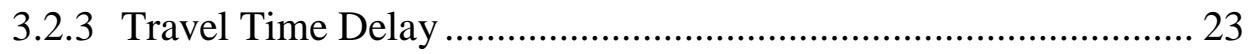

3.2.4 Total Expected loss .................................................................. 25

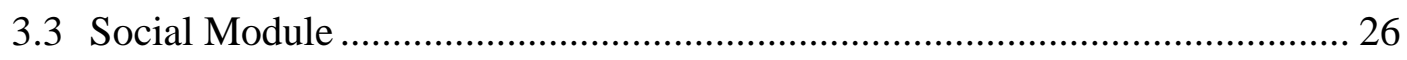

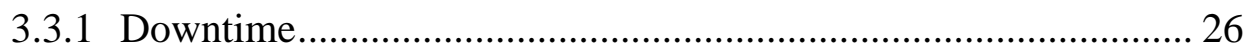

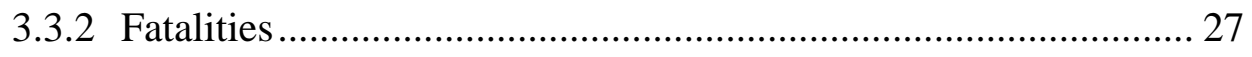

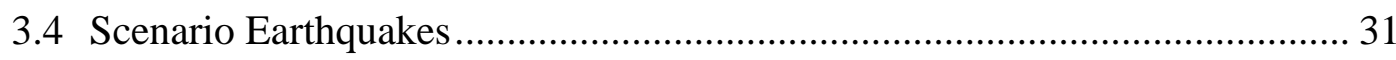

3.5 Seismic Vulnerability Assessment Model ........................................................ 33

3.6 Steps in Damage Algorithm for Bridges......................................................... 34

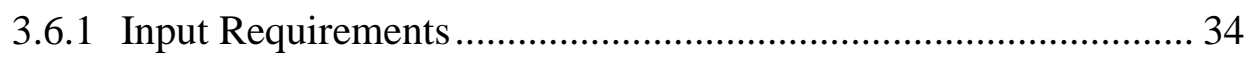

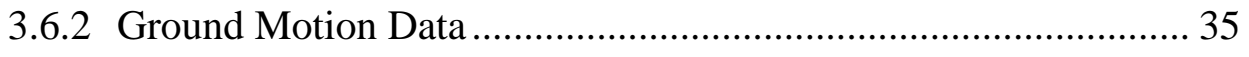

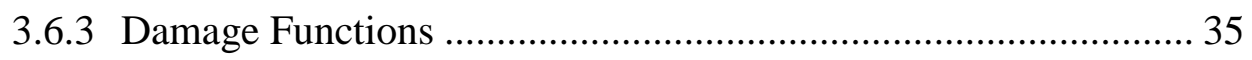

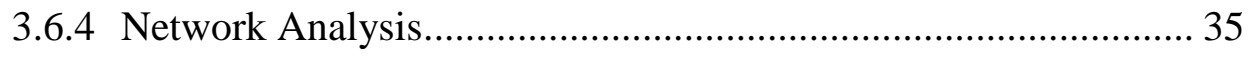

3.7 Seismic Vulnerability Assessment Results .................................................... 36

3.7.1 Cascadia Subduction Zone Earthquake near Northern Oregon

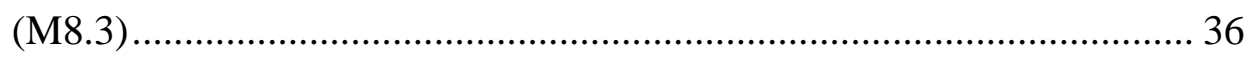

3.7.2 Cascadia Subduction Zone Earthquake near Southern Oregon

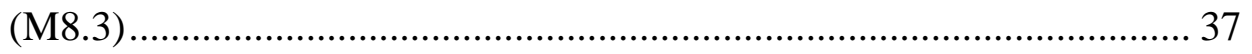

3.7.3 Cascadia Megathrust - Full length CSZ ……………………….... 38

3.7.4 Crustal Earthquake Scenario - Portland Hills Fault (M7.0).......... 41

3.7.5 Crustal Earthquake Scenario - Klamath Graben Fault System

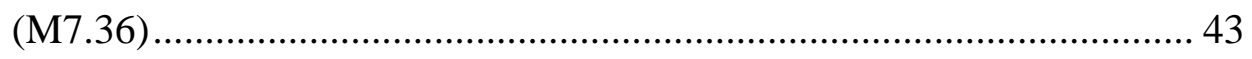

3.7.6 Crustal Earthquake Scenario - Mount Angel Fault (M6.80)......... 44

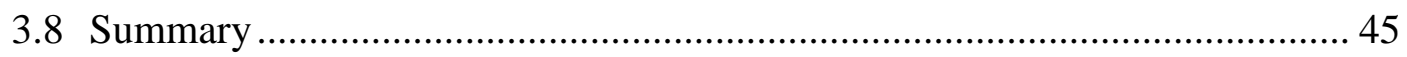

Chapter 4 Seismic Retrofitting for Square Reinforced Concrete Bridge Columns ...... 49 


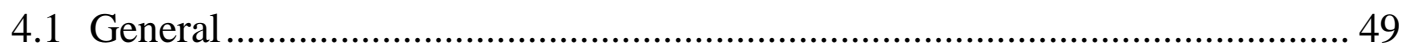

4.2 Selection of Representative Bridge type for Oregon ..................................... 50

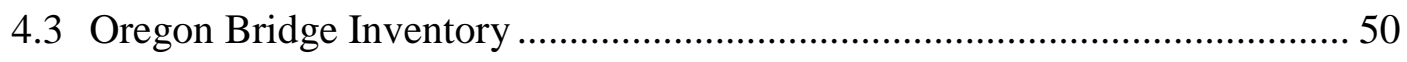

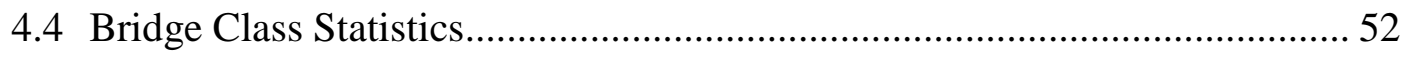

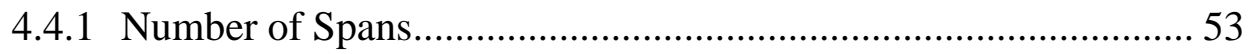

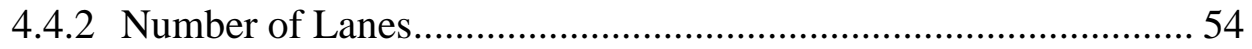

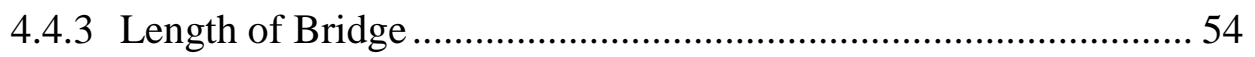

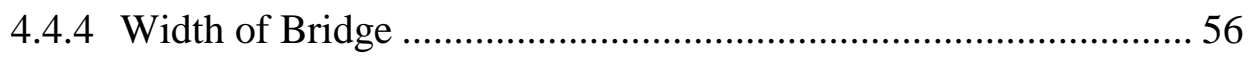

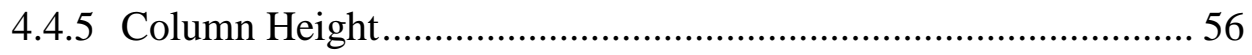

4.4.6 Number of Columns per bent....................................................... 57

4.4.7 Reinforcement Details ................................................................. 57

4.5 Representative Bridge Type for Oregon Inventory ........................................ 57

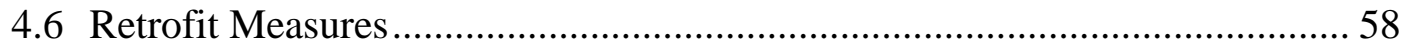

4.6.1 Retrofit Options for Deficient Columns ………………………... 59

4.6.2 Recommended Retrofit Option ................................................... 64

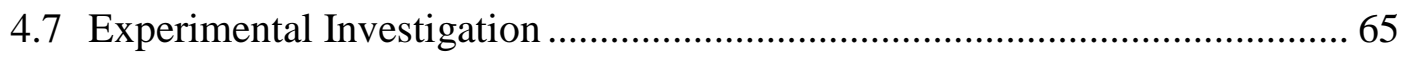

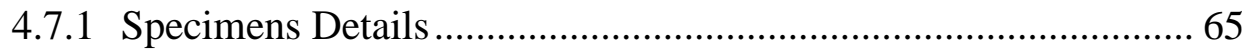

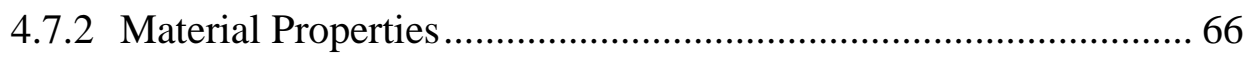

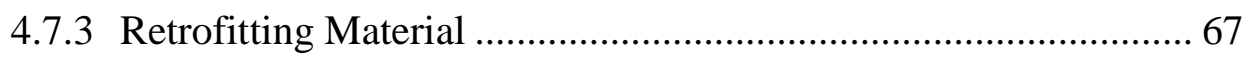

4.7.4 Test Setup and Load History ........................................................ 69

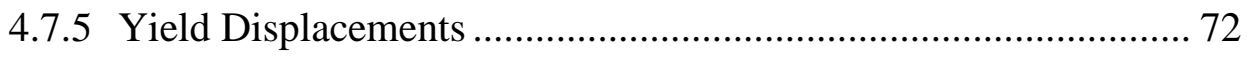

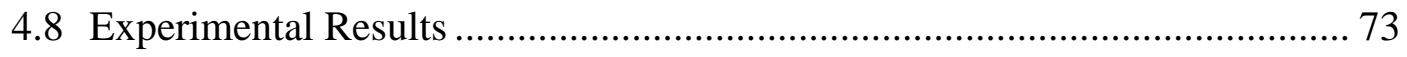

4.8.1 Column Displacement Capacities and Failure Modes ................... 74

4.8.2 Backbone Curve Comparison ................................................... 81 
4.8.3 Measured Curvature and Strain ................................................... 82

4.8.4 Stiffness Degradation and Cumulative Energy Dissipation........... 85

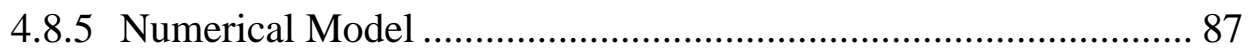

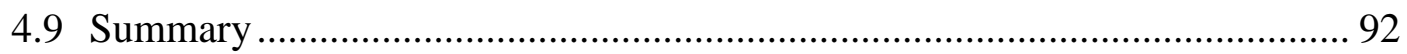

Chapter 5 Seismic Fragility Assessment ………………....................................... 94

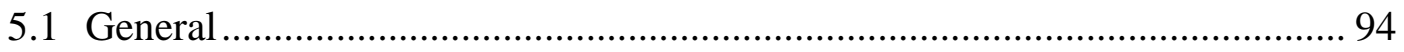

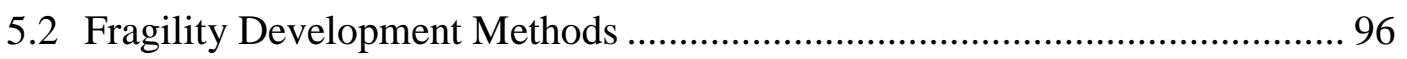

5.2.1 Empirical Methods...................................................................... 96

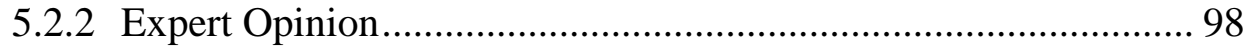

5.2.3 Analytical Methods ................................................................... 99

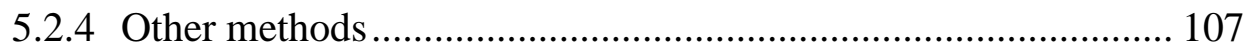

5.3 Fragility Curves for Retrofitted Bridges ........................................................ 108

5.4 Fragility Curve for a Representative Deficient Three-Span Concrete Continuous Girder Bridge........................................................................... 110

5.4.1 Bridge Model Description.......................................................... 111

5.4.2 Fragility Curve Development Methodology ………………….... 112

5.5 Fragility Curve for a Retrofitted Three-Span Concrete Continuous Girder

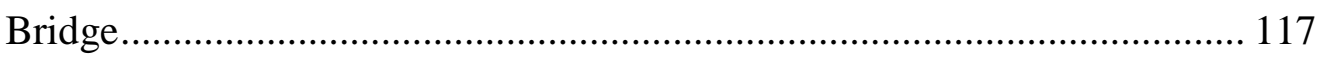

5.6 Fragility Sensitivity Analysis...................................................................... 122

Chapter 6 State of the Art on Prioritization of Bridges for Seismic Retrofitting ....... 124

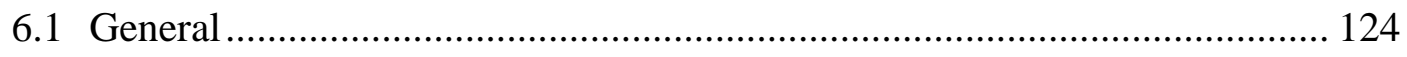

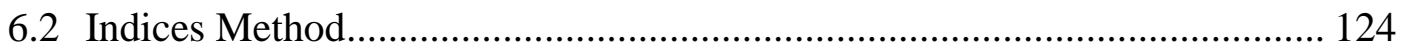

6.3 Expected Damage Method ........................................................................ 125

6.4 Seismic Risk Assessment Method .................................................................. 127 
6.5 Other Methods

6.6 Summary.

Chapter 7 Prioritization Application to Oregon's Highway Transportation Network 134

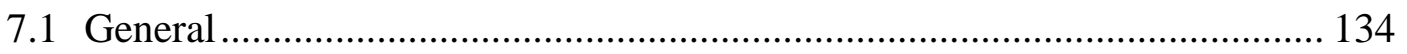

7.2 Cost-Benefit Analysis for Prioritization .............................................. 136

7.3 Economic Module ........................................................................... 137

7.3.1 Expected Loss ............................................................ 137

7.3.2 Cost-Benefit Ratio ....................................................... 138

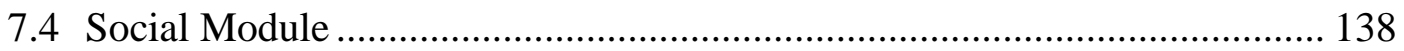

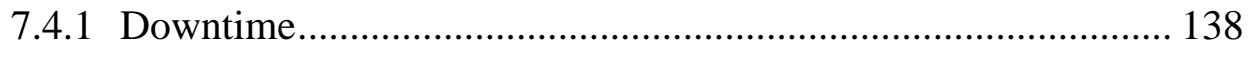

7.4.2 Fatalities .................................................................... 139

7.5 Cost-Benefit Assessment Results ................................................... 139

7.5.1 Assessing Benefit of Retrofit ........................................... 139

7.5.2 Retrofit Prioritization ................................................... 145

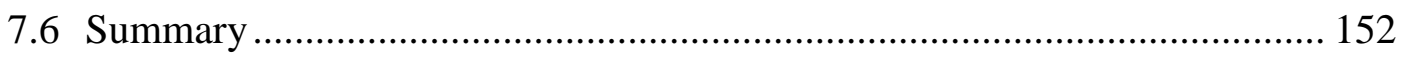

Chapter 8 Summary and Conclusions .......................................................... 153

8.1 Retrofit Measure ................................................................................ 153

8.2 Retrofit Prioritization ....................................................................... 153

8.2.1 Limitation in Seismic Risk Assessment Methodology .............. 154

8.2.2 Uncertainty in Earthquake Modeling................................. 155

8.2.3 Uncertainty in Bridge Modeling ....................................... 155

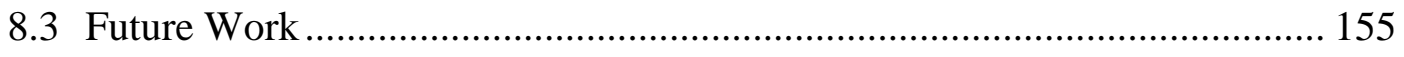

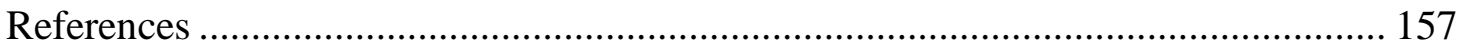

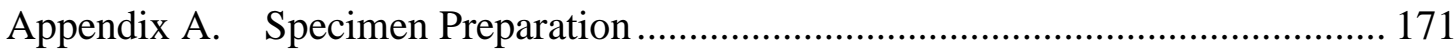


Appendix B. HAZUS Default Bridge Modelling Data...................................... 172

Appendix C. Seismic Risk Assessment per Highway Segment: Casuality ............ 175 


\section{LIST OF TABLES}

Table 2-1 Oregon Seismic Activity ...................................................................... 8

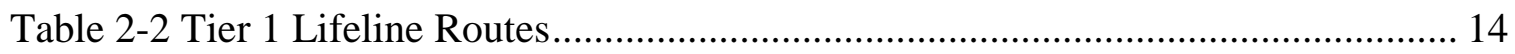

Table 2-3 Earthquake Lifeline Routes .................................................................. 15

Table 3-1 Damage States considered in HAZUS99-SR2 Bridge Model .......................... 20

Table 3-2 Repair Cost Estimate …………………………................................... 22

Table 3-3 Change in Link Capacity and Free Flow Speed ............................................. 24

Table 3-4 Continuous Restoration Functions for Highways (ATC-13, 1985)................. 26

Table 3-5 Discrete Restoration Functions for Highways (HAZUS-MH (FEMA, 2010)) 27

Table 3-6 Default Relationships for Estimating Population Distribution (HAZUS- MH4

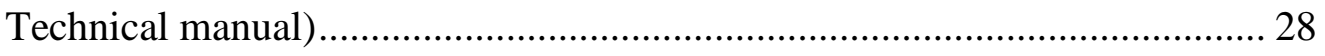

Table 3-7 Casualty Rates by Model Building Type for Complete Structural Damage (No Collapse) (HAZUS-MH Technical manual) ................................................... 30

Table 3-8 Injury Classification Scale (HAZUS-MH Technical manual) ......................... 30

Table 3-9 Summary of Damage States and Loss Estimates .............................................. 46

Table 3-10 Casualty Count per Severity Level............................................................. 46

Table 3-11 Cost Associated with Fatality, VSL (in Millions) ............................................ 47

Table 4-1 Construction Materials Listed in NBI (FHWA, 1995a)................................... 51

Table 4-2 Construction Types Listed in NBI (FHWA, 1995a)....................................... 51 
Table 4-3 Number of Bridges per Class 52

Table 4-4 Statistics of Number of Spans for major concrete bridge classes 53

Table 4-5 Column Ductility Summary 64

Table 4-6 Reinforcing Steel Properties 67

Table 4-7 Experimental Program Test Matrix and Measured Material Strengths 70

Table 4-8 Summary of Theoretical and Experimental Results 80

Table 5-1 Damage states for fragility curve development (Bazaez et al. 2015) 111

Table 5-2 Fragility curve values for representative as-built MSCG bridge bent (Bazaez \& Dusicka, 2018) 114

Table 5-3 Fragility Curve values for Conventionally Designed 3-Span Bridge (FEMA, 2010) 115

Table 5-4 Fragility curve values for representative as-built MSCG bridge bent (Choi, DesRoches, \& Nielson, 2003). 115

Table 5-5 Damage/limit state of bridge components (adapted from Dutta and Mander, 1999) 117

Table 5-6 Damage states for retrofitted bridge 118

Table 5-7 Fragility curve values for CFRP retrofitted RC bridge column 119

Table 5-8 Fragility Curve values for Seismically Designed MSCG Bridge (FEMA, 2010) 120 
Table 5-9 Fragility curve values for CFRP retrofitted MSCG bridge bent (Billah, Alam, \& Bhuiyan, 2013) 120

Table 7-1 Damage Comparison for As-built and Retrofit of Selected Bridge Types..... 143

Table 7-2 Damage Induced Cost for Before and After Retrofit of all MSCG Bridges (in Millions) 143

Table 7-3 Damage Induced Cost for Before and After Retrofit of all MSCG and MSSC Bridges (in Millions) 143

Table 7-4 Retrofit Prioritization Results for CSZ M9.0 (a) 146

Table 7-5 Retrofit Prioritization Results for CSZ M9.0 (b) 147

Table 7-6 Retrofit Prioritization Results for CSZ M9.34 148

Table 7-7 Retrofit Prioritization Results for Portland Hills M7.0 149

Table 7-8 Retrofit Prioritization Results for Klamath Falls M7.36 150

Table 7-9 Retrofit Prioritization Results for Mt. Angels M6.8 151

Table 7-10 Summary of Seismic retrofit Prioritization of Routes 152 


\section{LIST OF FIGURES}

Figure 2-1 Seismic Event Time-line (Basoz \& Kiremidjian, 1996).................................. 5

Figure 2-2 Known Faults in Oregon (Oregon Dept. of Geology and Mineral Industries) . 8

Figure 2-3 Illustration of seismic loading consideration over the years......................... 10

Figure 2-4 Oregon Seismic Lifeline Routes (CH2MHILL, 2012) .................................. 13

Figure 3-1 Restoration Curves for Highway Bridges (ATC-13, 1985) ……………....... 27

Figure 3-2 Selected ShakeMap Scenarios Developed (USGS): (a) CSZ M9.34 (b) Portland Hills M7.0 (c) Klamath Falls M7.36, and (d) Mt. Angels M6.8..................... 33

Figure 3-3 Study Area ........................................................................................... 34

Figure 3-4 CSZ M8.3 North Scenario PGA Distribution and Component Damage States

Figure 3-5 CSZ M8.3 South Scenario PGA Distribution and Component Damage States 38

Figure 3-6 CSZ 9.0 - Scenario (1) PGA Distribution and Component Damage States ... 39

Figure 3-7 CSZ 9.0 - Scenario (2) PGA Distribution and Component Damage States ... 40

Figure 3-8 CSZ 9.34 Scenario PGA Distribution and Component Damage States 41

Figure 3-9 Portland Hills Fault - Scenario (1) PGA Distribution and Component Damage States 42

Figure 3-10 Portland Hills Fault - Scenario (2) PGA Distribution and Component Damage States 43 
Figure 3-11 Klamath Fault Scenario PGA Distribution and Component Damage States 44

Figure 3-12 Mt Angels Fault Scenario PGA Distribution and Component Damage States

Figure 4-1 PMF of multi span MSCG bridges built before 1990 ................................. 53

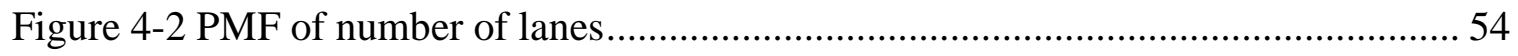

Figure 4-3 Cumulative Distribution Function for the Lengths ...................................... 55

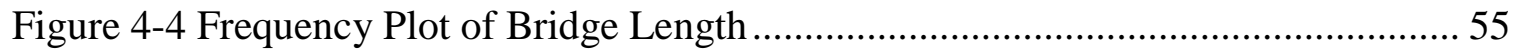

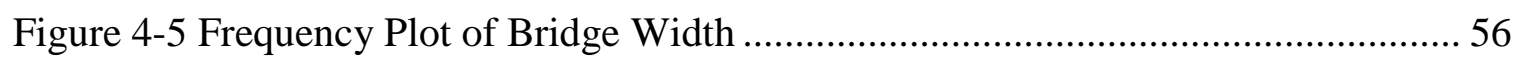

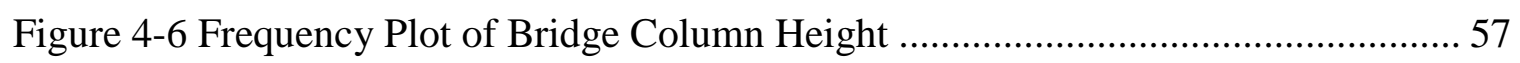

Figure 4-7 Details of a typical circular column steel jacket (Wright, Desroches, \& Padgett,

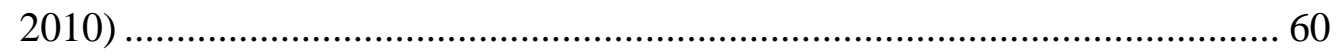

Figure 4-8 Details of a typical rectangular and circular column retrofitted with a steel jacket

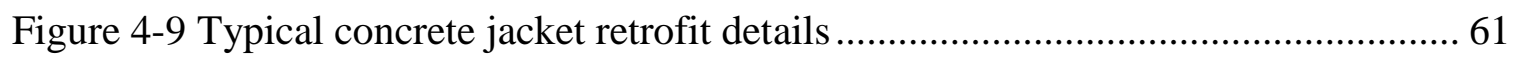

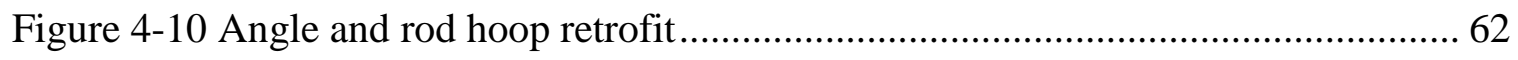

Figure 4-11 Elevation and cross-section of external prestressing steel retrofit.............. 63

Figure 4-12 (a) Test setup and reinforcement details; (b) instrumentation details ........... 66

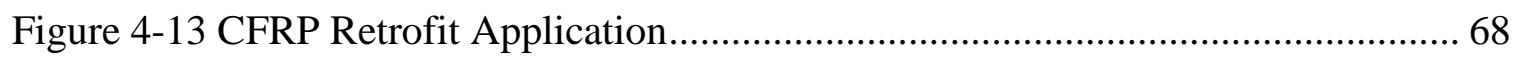

Figure 4-14 (a) Conventional Loading Protocol; (b) Subduction Loading Protocol ...... 71 
Figure 4-15 Hysteretic curves of (a) A-C; (b) A-S; (c) R-S; (d) Backbone Comparison. 77

Figure 4-16 Observed Damage (a) A-C; (b) A-S; (c) R-S ............................................. 78

Figure 4-17 Base rotation versus displacement ...................................................... 79

Figure 4-18 Average curvature profiles (a) A-C, (b) A-S, (c) R-S .................................. 83

Figure 4-19 Strain profiles (a) A-C, (b) A-S, (c) R-S .................................................. 85

Figure 4-20 (a) Stiffness degradation; (b) cumulative energy dissipation ....................... 87

Figure 4-21 Numerical model...................................................................................... 90

Figure 4-22 Experimental vs numerical results. (a) A-C, (b) A-S ................................... 91

Figure 5-1 Fragility Curve Example ……………………...................................... 94

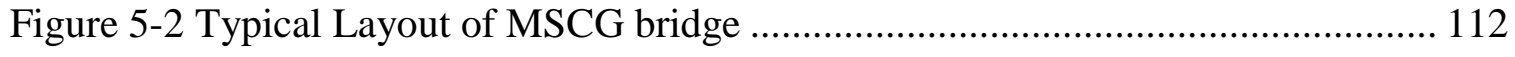

Figure 5-3 Fragility curve values for representative as-built MSCG bridge bent (Bazaez \&

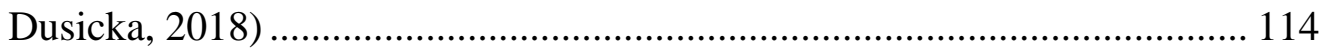

Figure 5-4 Fragility Curves for Conventionally Designed 3-Span Multi-Span Continuous

Concrete Bridge (FEMA, 2010) ................................................................ 115

Figure 5-5 Fragility curves for representative as-built MSCG bridge (Choi, DesRoches, \&

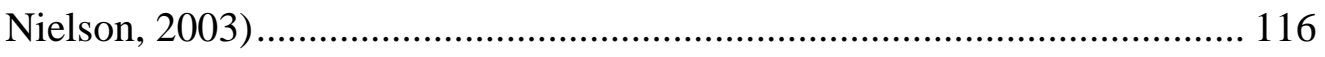

Figure 5-6 Fragility curve comparison for multi span concrete columns (a) Slight; (b) Moderate; (c) Extensive; (d) Collapse damage States ................................... 116

Figure 5-7 Fragility Curves for CFRP Retrofitted MSCG Column................................. 119

Figure 5-8 Fragility Curves for Seismically Designed MSCG Bridge (FEMA, 2010) .. 120 xvii 
Figure 5-9 Fragility curve for CFRP retrofitted MSCG bridge bent (Billah, Alam, \& Bhuiyan, 2013) 121

Figure 5-10 Fragility curve comparison for retrofitted columns (2018) (a) Slight; (b) Moderate; (c) Extensive; (d) Collapse damage States 121

Figure 5-11 Sensitivity Analysis, (a) CSZ M9.34 Scenario (MSCG and MSSC), (b) Portland Hills M7 (MSCG and MSSC), Scenario, (c) CSZ9 M9.34 Scenario (all bridges built <1990), and (d) Portland Hills M7 Scenario (all bridges built $<1990)$

Figure 7-1 Schemathic of Cost-benefit Analysis for Prioritization 137

Figure 7-2 Fragility curve comparison for as-built and retrofitted columns (a) Slight; (b) Moderate; (c) Extensive; (d) Collapse damage States 141 


\section{CHAPTER 1}

\section{INTRODUCTION}

\subsection{Research Motivation}

The majority of Oregon's bridge inventory was built prior to the current understanding of bridge response and prior to current understanding of the expected earthquake demands. While some bridges are being replaced due to other deficiencies, the majority are expected to continue to stay in service for decades to come. Oregon's vulnerable and seismically deficient old bridges need to be retrofitted. However, it is economically not feasible to retrofit the entire seismically deficient bridges. Hence, a prioritization method is needed to aid bridge investment decisions and for allocating of the limited resources available.

\subsection{Objectives and Scope of Research}

The goal of this study is to assess and refine bridge prioritization methodology to be utilized for ranking Oregon's bridge inventory. Although there are many retrofit prioritization methodologies out there, the majority focus on ranking individual bridges. Oregon department of transportation, however, intend to focus on retrofitting an entire highway segment. The level of detail that is used in the refinement and development of this methodology will aid decision makers understand the vulnerability of the current state of the bridges and use a similar framework for prioritization for hazards other than seismic forces.

The specific tasks that will be completed as part of this research are as follows: 
- Assess the vulnerability of Oregon's highway bridge network.

- Recommend economical retrofitting technique and demonstrate the effectiveness through experimental and analytical evaluation of deficient reinforced concrete bridge columns subjected to subduction zone earthquake, in their as-built and retrofitted state.

- Develop representative seismic fragility curves for typical bridge in Oregon that describe the conditional probability of exceeding a level of direct or indirect bridge damage for a given level of seismic hazard, in their as-built and retrofitted states.

- Develop a prioritization methodology that focuses on assessing the vulnerability of highway segments under seismic loading.

- Present ranking of highway segments for seismic retrofitting when earthquake ground motion is the only hazard considered.

\subsection{Dissertation Outline}

This thesis is organized into seven chapters with the following contents:

Chapter 1 discusses the motivation and significance of study, objectives and scope of research, methodology.

Chapter 2 gives the background in the vulnerability and importance of Oregon's highway bridge network. The state of Oregon's ageing bridge inventory is discussed in light of seismic hazard sources in the study area. Moreover, lifeline routes that are selected by Oregon Department of Transportation. 
Chapter 3 seismic vulnerability of Oregon's bridges and network is evaluated and results of seismic risk assessment of the current state are presented.

Chapter 4 summarizes literature review on conventional retrofit measures for deficient reinforced concrete bridge columns. The chapter then discusses the retrofit technique that was selected. Further, columns were evaluated experimentally to understand their cyclic behavior and the effectiveness of retrofit technic by aiming to represent displacement demands imposed in bridges by subduction zone earthquakes will be presented. Before retrofit options were studied, a representative bridge type from Oregon's inventory is selected. The process of selecting of a representative bridge is also presented.

Chapter 5 presents fragility curves for the as-built and retrofitted condition of a representative bridge. A literature review of methods in the development of fragility curves is also presented.

Chapter 6 presents a state-of-the art on prioritization of bridges for seismic retrofitting.

Chapter 7 presents the methodology and results of prioritization of lifeline segments for seismic retrofit. The section also presents the benefit of a retrofit by comparing to the expected present value of the losses without retrofit and present value of the losses with retrofit. A Cost-Benefit Analysis will be performed and the highway segments will be ranked according to largest expected savings in losses over the remaining life per amount of money invested in retrofitting. 
Finally, in Chapter 8, a summary and conclusions are drawn from the research, and future research needs are outlined. 


\section{CHAPTER 2}

\section{VULNERABILITY AND IMPORTANCE ASSESSMENT}

\subsection{Background}

Hazard vulnerability assessments are very important in disaster preparedness and prevention. Natural disasters could be events such as a flood, an earthquake, or a hurricane that causes great damage or loss of life. Large-scale events can cause serious disruptions of the function of a society and involve human life, economic or environmental losses or impacts. Disasters are the product of a combination of hazards and vulnerability. Hence, in order to minimize losses and disruptions caused by a seismic event, risk assessment is key so mitigation steps can be taken to address the vulnerability. Figure 2-1 shows a schematic showing components of disaster management program developed by Basöz \& Kiremidjian (1996).

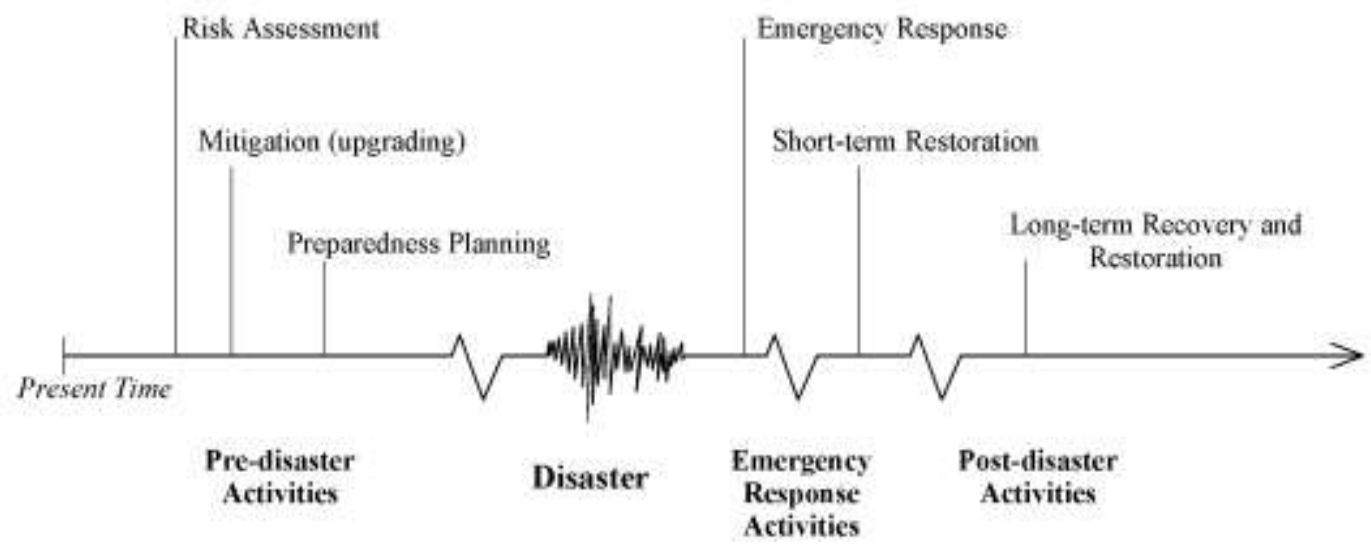

Figure 2-1 Seismic Event Time-line (Basoz \& Kiremidjian, 1996).

Pre-disaster activities include risk assessment, mitigation and preparedness

planning. Evaluation of the hazard, structures vulnerability and impact is risk assessment, 
and results of the assessment are used in mitigation and planning actions. Mitigation includes designing resilient structures and retrofitting existing ones to improve their performance and help reduce vulnerability of structures to a hazard. The following section cover the vulnerability of Oregon's bridges and seismic risk assessment done to determine preventative actions taken before a seismic event to alleviate consequences.

\subsection{Seismic Hazard Characterization}

In the Pacific Northwest, earthquakes result from slip on faults in a variety of geographic and geologic settings. The Juan de Fuca Oceanic Plate is slipping steadily beneath the North America Continental Plate at a rate of several $\mathrm{cm}$ per year stresses that are consequential to earthquake in the area. This area is what is called the Cascadia Subduction Zone, which extends from northwestern California through western Oregon and western Washington to Vancouver Island, Canada. The relative plate motion of the Juan de Fuca and the North American plate is accommodated by deformation of the latter plate. Therefore, the earthquakes in the area are associated with both the subduction process and the deformation of the North America plate.

The different modes of earthquake occurrence in Washington, Oregon, and the offshore continental slope include "Megathrust" earthquakes, "Crustal" earthquakes, "Deep" earthquakes, and "Volcanic" earthquakes. "Megathrust" earthquakes result from rupture of the boundary between the subducting Juan de Fuca plate and the overriding North America plate, while "Crustal" earthquakes originate from slip on faults within the crust of the North American Plate, whereas "Deep" earthquakes that result from faulting 
within the down-going Juan de Fuca Plate, and "Volcanic" earthquakes are associated with volcanic processes.

In 1984, seismologists proposed that Cascadia might produce subduction type earthquakes as Japan. Researchers have since confirmed that Cascadia has a long history of great subduction earthquakes with the most recent, magnitude 9.0 event, having had occurred on January 26, 1700 AD (Atwater, B. F., Musumi-Rokkaku, K. Satake,, Ueda, \& Yamaguchi, 2005). A decade later, the Oregon's building codes were updated to address this newly revealed earthquake threat. The energy for the next great earthquake is currently building along the fault. And according to the ten thousand records of past Cascadia seismic events assembled by geologists, intervals have been highly variable and vary in size and location. The magnitudes range from 8.3-9.3 and extend from British Columbia to Northern California.

The map on Figure 2-2 shows only the known faults in Oregon. Faults that have moved most recently are shown in red. Furthermore, geologists are discovering, shallow, active earthquake faults all over Oregon and Washington State. These earthquakes, collectively, may present a higher risk than a Cascadia subduction zone event and Table 2-1 provides a brief summary of the primary earthquake sources affecting Oregon, their approximate frequency of occurrence, range of magnitude and most recent activity. 
Table 2-1 Oregon Seismic Activity

\begin{tabular}{llll}
\hline Source & Magnitude & $\begin{array}{l}\text { Frequency } \\
\text { (years) }\end{array}$ & Latest Occurrence \\
\hline \hline \multirow{2}{*}{ Crustal } & $\mathrm{M}<5.5$ & $15-20$ & Annually \\
\cline { 2 - 4 } & $\mathrm{M} \geq 5.5$ & Unknown & $\begin{array}{l}\text { March, 1993: M5.6 Scotts Mills } \\
\text { September, 1993: M5.9 \& M6.0 } \\
\text { Klamath Falls }\end{array}$ \\
\hline CSZ & $\mathrm{M} \geq 8.0$ & $350-500$ & January, 1700 \\
\hline Interplate & $\mathrm{M}=4.0-7.0$ & $30-50$ & February 2009: M4.1 Grants Pass \\
\hline
\end{tabular}

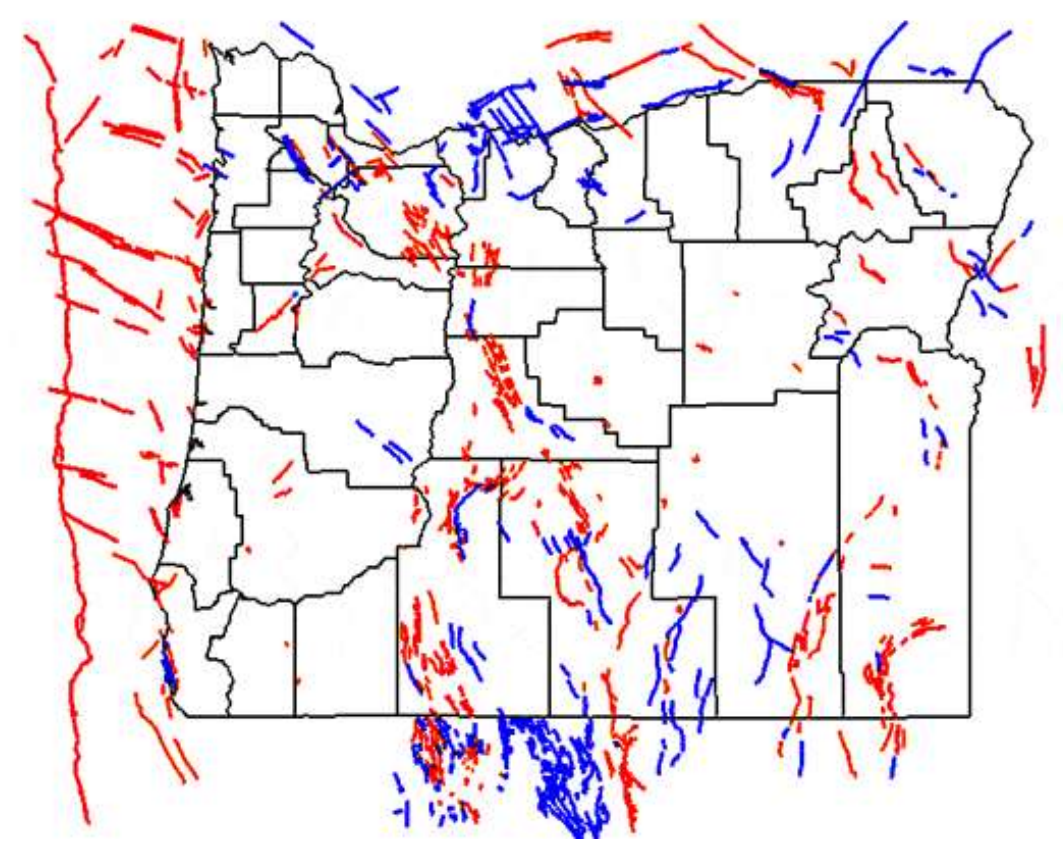

Figure 2-2 Known Faults in Oregon (Oregon Dept. of Geology and Mineral Industries)

\subsection{Seismic Vulnerability Evaluation}

Vulnerability is a function of the site hazard and the structural properties of the bridges. Oregon has the potential for a 9.0+ magnitude earthquake caused by the Cascadia Subduction Zone. The effects of an earthquake of this magnitude can result in 
potential sudden detrimental impact on the transportation infrastructure where bridges represent vulnerability points within the network. When bridge damage occurs during a seismic event, short-term or long-term interruptions to traffic flow result. This will delay emergency response in the hours after the event, and restrict the movement of people and goods for months. Hence, the economic impact of bridge damage includes not only the cost of structural repair, but also longer term consequences relating to valued loss of time when commuter and freight travel slows down to navigate the disrupted network.

Cascadia's earthquake potential has only become known over the last few decades, hence, much of the infrastructure supporting the community were built without taking this seismic hazard into account. According to the 2010 ODOT inventory, there are close to 10,000 bridges and culverts. Over $80 \%$ of the bridges in the inventory were built before 1990 of close to $70 \%$ of those were built before 1970 , before which bridges were designed primarily for gravity loads without much consideration to lateral forces from seismic loading as illustrated in Figure 2-3. With a majority of multi-span state owned bridges designed and built between 1950 and 1980, the state of Oregon would face a devastating post-earthquake situation if a major event occurred in the state. 


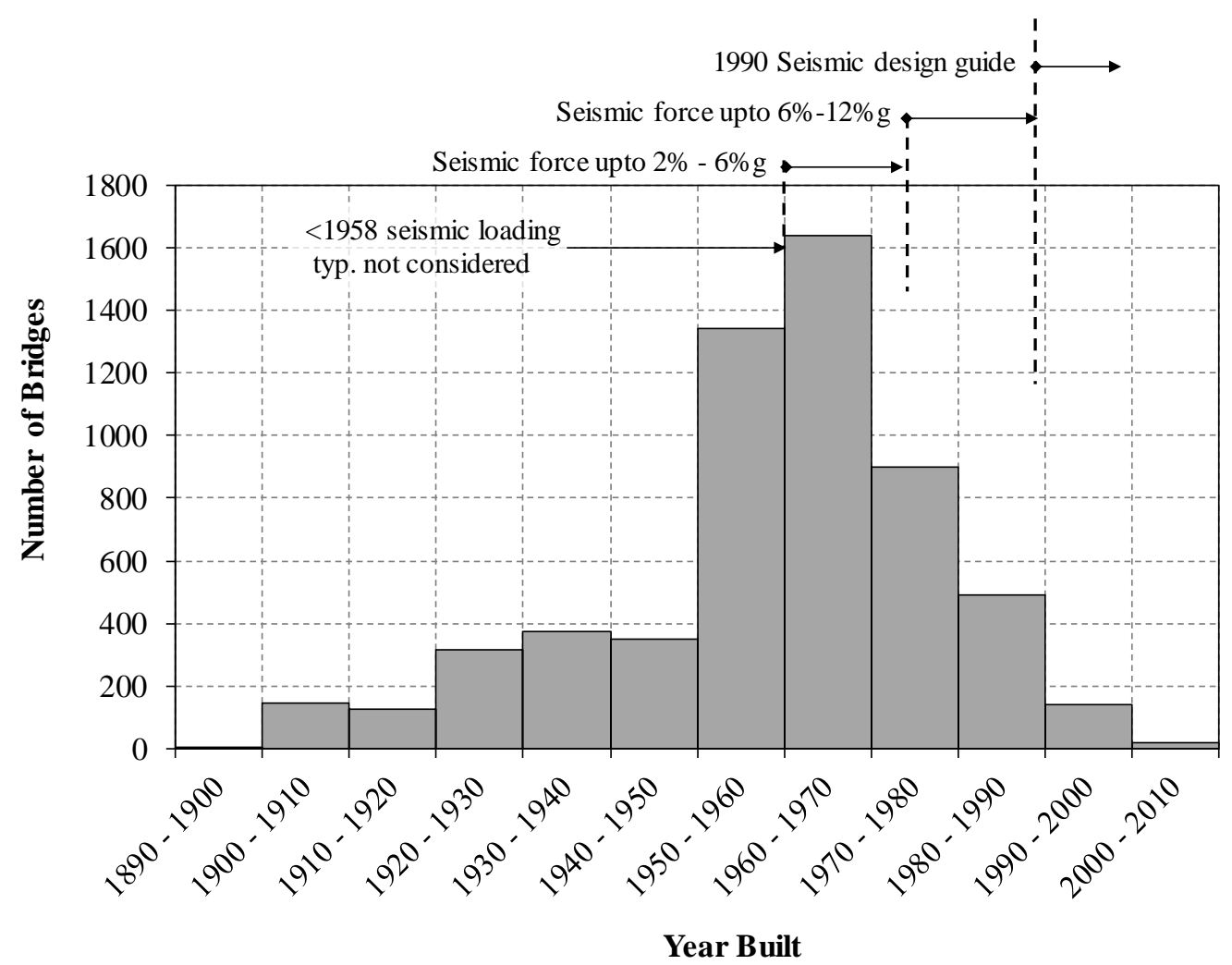

Figure 2-3 Illustration of seismic loading consideration over the years.

Hundreds of bridges in the State of Oregon are still vulnerable to earthquake damage. Over the last decade it has been shown during the course of bridge inspections that many of the bridges are showing signs of deterioration. As reported by Patrick Brennan for the Oregon Legislative Committee Services council in a brief, the causes of this deterioration problem include older construction methods, structures beyond their intended construction life, and a scale of increased use that was not accounted for in the original design of the bridges. Currently, the Oregon Department of Transportation (ODOT) owns and maintains just over 2600 bridges distributed over the state controlled routes. Of the approximately 2600 bridges, a fifth of them are beyond the 50-year construction life (Brennan, 2004). 
In the 2009 exercise that Oregon's office of Emergency Management conducted to assess the State's emergency response to a 9.0 magnitude earthquake on the Cascadia Subduction Zone, it was apparent that the effects of an earthquake of this magnitude was widespread across the most dynamic portion of the transportation network. In addition to the heavily damaged along US101, many portions of I-5 and US99 would be out of service as would most state routes connecting I-5 with the Oregon Coast. It could take anywhere between 3 to 12 months to restore traffic using temporary bridges. However, the restoration of the entire transportation network could take 3 to 5 years and would only be possible with a nationwide effort because of the limited workforce and resources availability within Oregon (Nako, et al., 2009).

\subsection{Importance Assessment}

The 1999 Oregon Highway Plan, Policy 1E, Lifeline Routes states, "It is the policy of the State of Oregon to provide a secure lifeline network of streets, highways, and bridges to facilitate emergency services response and to support rapid economic recovery after a disaster". In order to implement the said policy, ODOT conducted Oregon Seismic Lifeline Route Identification project. In the study done by CH2MHILL (2012), the project identified specific list of highways and bridges to make up the seismic lifeline system. They identified three critical goals that the routes are required to accomplish to capture the need immediately and short-term needs after the event, midterm needs after the event and long-term needs after an event. These goals are -

- Support survivability and emergency response efforts immediately following the event 
- Provide transportation facilities that are critical to life support functions for an interim period following the event

- Support statewide economic recovery

Specific objectives were also identified for each goal with a list of categories to measure how well the goal of each segment can be achieved. Moreover, specific parameters were also identified to measure each criterion. Using this evaluation framework, the project management team identified a three-tiered seismic lifeline system. Tier 1 is the highest priority segment and provides access to and through from central Oregon, Washington and Oregon. Whereas Tier 2 covers additional roadway segments that extend the range of Tier 1 system through all seismically vulnerable areas and provide redundancy in the Portland Metro and Willamette Valley. Moreover, Tier 3, the third highest priority, provide an interconnected network in conjunction with Tier 1 and Tier 2 segments. Figure 2-4 shows these proposed seismic lifeline routes with tier designations. 


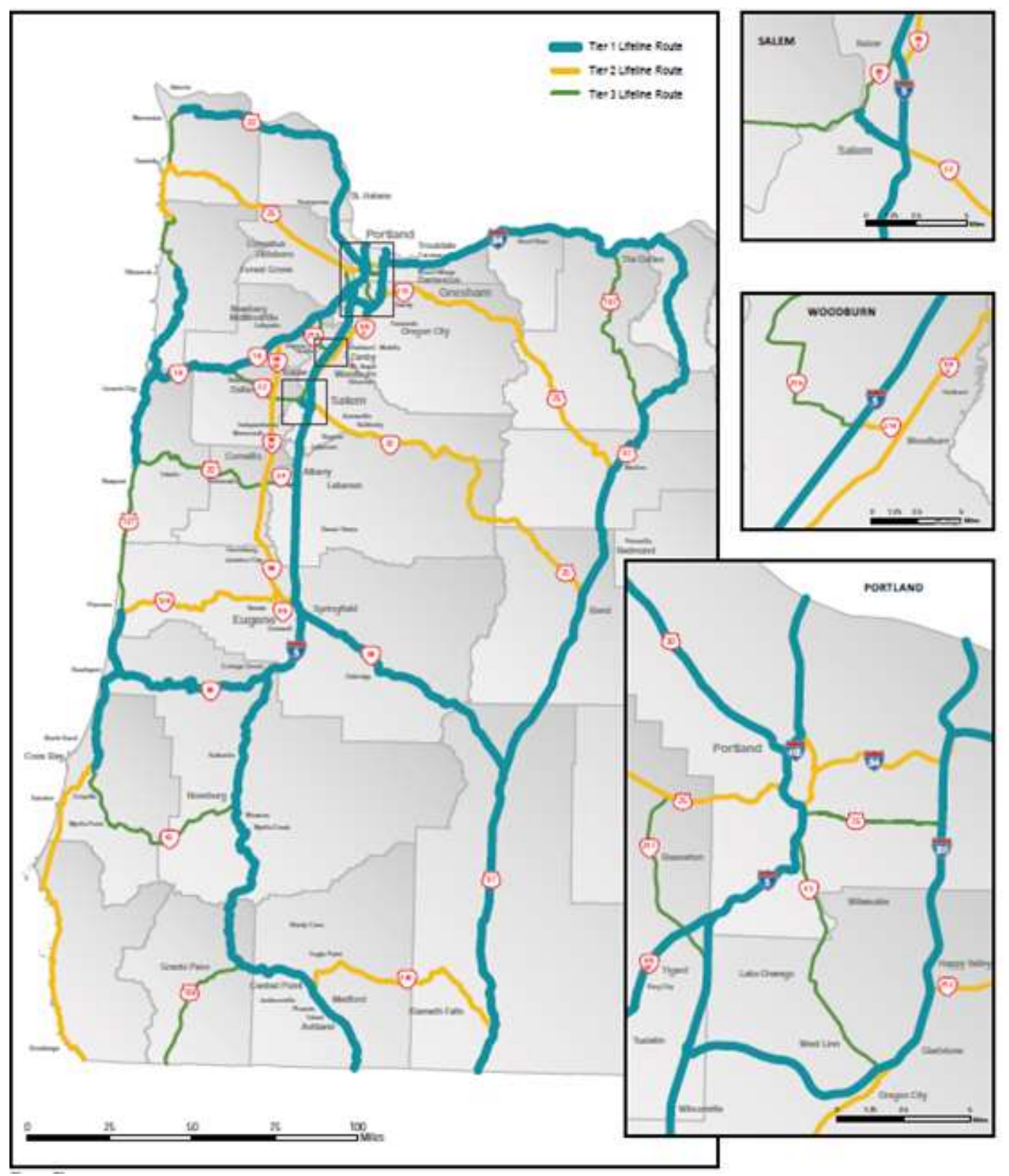

Figure 2-4 Oregon Seismic Lifeline Routes (CH2MHILL, 2012)

The routes identified as Tier 1 (Table 2-2) are considered the most significant and necessary to provide a functioning statewide transportation system. Routes were largely selected based on their likelihood of being available following a seismic event. 
Consequently, routes with fewer vulnerable bridges were often selected as a lifeline route instead of higher volume parallel routes with many vulnerable bridges. These lifeline routes were key in the prioritization done by $\mathrm{CH} 2 \mathrm{MHill}$ but were not intended for emergency scenarios. ODOT has since updated earthquake lifeline routes and want future prioritization to include a corridor strategy. Hence, this study will consider the updated earthquake lifeline routes in the assessment of a prioritization methodology for retrofit. These routes are presented in Table 2-3.

Table 2-2 Tier 1 Lifeline Routes

\begin{tabular}{cc}
\hline Highway & Geographic Zone \\
\hline \hline I-5 & Portland Metro to South I-5 \\
\cline { 2 - 2 } & Cascades \\
\hline I-205 & Central \\
\hline I-405 & Portland Metro \\
\hline OR 99W & Portland Metro \\
\cline { 2 - 2 } & Portland Metro \\
\hline OR 18 & Valley \\
\hline US 30 & Valley \\
\hline US 101 & Coast \\
\hline US 97 & Coast \\
\hline OR 38 & Coast \\
\hline OR 58 & Central \\
\hline OR 22 & Coast \\
\hline
\end{tabular}


Table 2-3 Earthquake Lifeline Routes

\begin{tabular}{cc}
\hline Highway & Geographic Zone \\
\hline \multirow{2}{*}{ I-5 } & Portland Metro \\
\cline { 2 - 2 } & Valley \\
\cline { 2 - 2 } & South I-5 \\
\cline { 2 - 2 } I-84 & Cascades \\
\hline I-205 & Central \\
\hline I-405 & Portland Metro \\
\hline US 30 & Portland Metro \\
\hline US 101 & Coast \\
\hline OR 38 & Coast \\
\hline OR 42 & Coast \\
\hline US 20 & Coast \\
\hline US 26 & Coast \\
\hline
\end{tabular}

\subsection{Seismic Risk Assessment}

The earthquake hazard assessment provides local, state and regional officials with a decision support tool for estimating potential losses from scenario earthquakes. Being able to estimate this gives users that capability to anticipate the consequences of future earthquakes and to develop plans and strategies for reducing risk. The Seismic Risk Analysis (SRA) methodology is a synthesis of models developed by earth scientists, geotechnical and structural earthquake engineers, transportation engineers and planners, and economists. The methodology can develop multiple types of results from deterministic or probabilistic approaches and from local to large geographic areas. Such results can be developed for use in pre-earthquake assessment of various options for seismic risk reduction after an actual earthquake. In addition, the risk associated with 
earthquake hazards on highway systems is largely dependent on the complexity and redundancy of a network in providing smooth traffic flow. Seismic Risk Assessment studies can provide decision makers with an appreciation of the importance of having a highway network resistant to earthquakes and information to make the network invulnerable to these events.

\subsubsection{Previous Research on Seismic Vulnerability Assessments}

Seismic Design Decision Analysis (SDDA) is a methodology that was introduced by Whitman et al. in (1975). Most seismic risk assessments that are in used have been based on this methodology. SDDA considers the effects of earthquake hazard, damage, and also economic losses. The effects of the damages are studied as probabilities of different damage levels.

Earthquake damage to highway components can go well beyond life safety risks and the costs to repair the component itself. When bridge damage occurs during a seismic event, short-term or long-term interruptions to traffic flow result. This can impact postearthquake emergency response, repair and reconstruction. The level of impact depends on the seismic performance of individual components and the characteristics of the highway system such as network configuration, location, redundancy, traffic capacity and traffic volume (Werner S. D., et al., 2006).

Werner and Taylor (2002) emphasized the significance of observing component functionality and location within a lifeline system to assess system performance. Component functionality depends on seismic response characteristics of a component and the state of damage, and also how the damage can be repaired, cost of repair and its 
significance in the overall system. Knowing whether a bridge will be fully closed, partially open, or fully open provides a means of analyzing networks as a whole.

One of main end results from SRA of roadway systems is the estimation of economic impacts of earthquake damage to the system. Recent studies done on transportation networks place a strong emphasis on indirect costs due to traffic flow and travel times. Indirect economic loss estimate due to damaged bridges within the highway system from an earthquake event for Saint Louis was performed by Enke et al. (2008) their results showed that the indirect loss is significant when compared to the direct loss resulting from bridge damage.

The scope of seismic risk assessment gets larger as new methodologies for seismic risk analysis that provide a basis for developing mitigation plans and policies, emergency preparedness, and response and recovery planning are accessible. Powerful risk assessment software like HAZUS and REDARS2 produce estimates of hazardrelated damage and loss estimates before or after a disaster occurs. Recent studies done on transportation networks place a strong emphasis on indirect costs due to traffic flow and travel times. A study by Stevanovic and Nadimpalli (2010) presents the impact of degree of damage on the traffic in terms of user delay costs and determine how the earthquake damage influence traffic in terms of AM peak, mid-day, PM peak, and off peak traffic. The study found some links that are susceptible to damage on one scenario are critical in carrying detour traffic to other scenario. The cost estimate indicated that the maximum impacts would be imposed on PM traffic. 
Dusicka et al. (2007) developed a GIS model of the roadway and bridge network using new technology developed for the Federal Highway Administration called REDARS2. The research project concentrated on the development of a strategy to prioritize bridges on Oregon's freight routes for seismic retrofit. The data compiled has provided a strong foundation to this research. The following chapter will present a comparable SRA of the bridges in the Oregon highway network given various ground motion hazards.

\subsection{Summary}

Vulnerability is a function of the site hazard and the structural properties of the bridges. Historical records show that Oregon is earthquake-prone. In 1993 western Oregon experienced the Scotts Mills (magnitude 5.6) and Klamath Falls (magnitudes 5.9 and 6) earthquakes that were damaging. And the next subduction zone earthquake is expected to unleash its full potential, and is expected to be the worst natural disaster in U.S. history. In addition, majority of Oregon's bridges were built without much consideration to lateral forces from seismic loading as they were designed primarily for gravity loads. This combinations makes Oregon's transportation network vulnerable. The following chapter presents the steps followed to assess the seismic vulnerability of Oregon's bridges. 


\section{CHAPTER 3}

\section{SEISMIC VULNERABILITY ASSESSMENTS OF OREGON'S BRIDGES}

This study addresses only some of the many aspects of bridge seismic performance that may be important for assessing bridge damage states and repair requirements. Liquefaction induced damage is not included and the study focuses on damage induced from ground shaking.

\subsection{Damage Functions}

Basoz and Mander (1999) developed the HAZUS99-SR2 bridge model that defines, for generalized classes of bridges, capacities in terms of spectral acceleration leading to the onset of bridge damage state. These damage functions or fragility curves are modeled as lognormally distributed functions that give the probability of reaching or exceeding different damage states for a given level of ground motion or ground failure. The bridge model utilized for seismic risk assessment (SRA) of the Oregon transportation network, which is based on HAZUS99-SR2, defines bridge capacities in terms of spectral accelerations leading to the onset of five damage states as are listed in Table 3-1 for each of several "standard bridge" classifications.

For all the bridge types, fragility curves are defined in terms of $\mathrm{Sa}(0.3 \mathrm{sec}), \mathrm{Sa}$ (1.0 sec) and PGD. 28 "standard bridge" classes (HWB1 through HWB28) are derived for HAZUS. Bridges are classified according to seismic design, number of spans, structural material type, pier type, abutment type, and span continuity. The 28 classes differentiate between the various bridge characteristics in the National Bridge Inventory 
(NBI) and can be seen on Table 0-1 Basoz and Mander (1999) characterize each damage state for each of the 28 standard bridge classifications.

Table 3-1 Damage States considered in HAZUS99-SR2 Bridge Model

\begin{tabular}{l|l}
\hline $\begin{array}{l}\text { Damage State } \\
\text { Designation }\end{array}$ & Description of Typical Expected Damage \\
\hline \hline None & Up to first yield. \\
\hline Slight & $\begin{array}{l}\text { Minor cracking and spalling of the abutment, cracks in shear keys at abutment, } \\
\text { minor spalling and cracking at hinges, minor spalling of column requiring no more } \\
\text { than cosmetic repair, or minor cracking of deck. }\end{array}$ \\
\hline Moderate & $\begin{array}{l}\text { Any column experiencing moderate shear cracking and spalling (with columns still } \\
\text { structurally sound), moderate movement of abutment }(<5.1 \mathrm{~cm})(<2 \text { inches), } \\
\text { extensive cracking and spalling of shear keys, connection with cracked shear keys } \\
\text { or bent bolts, keeper bar failure without unseating, rocker bearing failure, or } \\
\text { moderate settlement of approach. }\end{array}$ \\
\hline Extensive & $\begin{array}{l}\text { Any column degrading without collapse (e.g., shear failure) but with column } \\
\text { structurally unsafe, significant residual movement of connections, major settlement } \\
\text { of approach fills, vertical offset or shear key failure at abutments, or differential } \\
\text { settlement. }\end{array}$ \\
\hline Complete & $\begin{array}{l}\text { Collapse of any column or unseating of deck spans leading to collapse of deck. } \\
\text { Tilting of substructure due to foundation failure. }\end{array}$ \\
\hline
\end{tabular}

\subsection{Economic Module}

The economic module comprises the value of investment on the highway bridges in the form of retrofits and expected losses after sustaining a seismic event. Loss estimation comprises of direct and indirect economic losses. The repair/replacement cost of damaged bridges is the direct economic loss. The loss due to drivers' delay is assumed to represent the indirect economic loss due to the disruption in the network in this preliminary economic study. Retrofit is a direct economic cost. 


\subsubsection{Cost of Retrofit}

ODOT's Bridge Program is tasked in the maintenance of freight mobility and preservation of existing high cost bridges and the funding does not include the retrofit of existing deficient bridges. This limits the level of retrofitting that can be done. Hence, ODOT has plans to address the retrofit needs in two stages. With Life safety as the fundamental goal, retrofit details that are designed to prevent the superstructure from separating from the substructure to preventing collapse of a span are considered. Though, this type of retrofit can be effective for moderate earthquakes, bridges may collapse in the event of a large earthquake.

Phase II retrofitting, on the other hand, includes strengthening the substructure elements such as caps, columns, footings and piling. Life safety is still the primary goal. And the retrofit is expected to provide life safety for a maximum expected earthquake. According to ODOT (2009), the cost of Phase II work is typically three times that of Phase I and are given in Equation 3-2.

$$
\begin{array}{ll}
\text { Retrofit Cost }(\text { Phase I })=\frac{\$ 35}{\mathrm{ft}^{2}} \times \text { the deck area } & \text { Equation 3-1 } \\
\text { Retrofit Cost }(\text { Phase II })=\frac{\$ 90}{\mathrm{ft}^{2}} \times \text { the deck area } & \text { Equation 3-2 }
\end{array}
$$

\subsubsection{Repair and Replacement Cost}

Bridge damage results not only in high cost of structural repair but also safety concerns by severely disrupting traffic flow which in turn will impact post-earthquake emergency response, repair and reconstruction operations and long term economic 
consequences due to the valued loss of time when commuter and freight travel slows down due to the disrupted network.

The SRA methodology employed in this study uses the bridge and network data to estimate direct and indirect economic losses due to disruption in the system. The SRA considers repair costs and losses due to earthquake-induced travel-time. The replacement cost in this study are calculated as a product of a base cost of $\$ 165 / \mathrm{ft}^{2}$, the deck area and a factor of 3.2 with a $\$ 3$ million minimum cost. And when estimating the cost of a new bridge with an old bridge, a further multiplication factor of 1.2 is used (Equation 3-3), because the new bridge is expected to be of a larger dimension than the old one (ODOT, 2009). The repair cost is computed as the product of a repair cost ratio and replacement cost. The repair cost ratio depends on the bridge's damage state as shown in Table 3-2.

Replacement cost is calculated as:

$$
\begin{array}{r}
\text { Replacement cost }=\max \left(\left(\frac{\$ 162}{\mathrm{ft}^{2}} \times \text { deck area }\left(\mathrm{ft}^{2}\right) \times 3.2 \times 1.2\right), \$ 3 \text { million }\right) \\
\text { Equation } 3-3
\end{array}
$$

Table 3-2 Repair Cost Estimate

\begin{tabular}{l|c|c}
\hline Damage State & Repair Cost Ratio & Min Cost \\
\hline \hline None & 0 & 0 \\
Slight & 0.03 & $\$ 100,000$ \\
Moderate & 0.25 & $\$ 500,000$ \\
Extensive & 1.0 & Min \$3 Million \\
Collapse & 1.0 & Min \$3 Million \\
\hline
\end{tabular}




\subsubsection{Travel Time Delay}

The performance of bridges will be integrated with transportation network in the context of seismic risk assessment. To define the transportation network performance, a comprehensive index of performance introduced by Shinozuka, et al. (2000) is used. This index is 'Drivers' Delay' and is described as the increase in total daily travel time for drivers due to earthquake induced delays. Drivers' Delay is calculated as the difference between the total daily travel for all network travelers on the damaged network and that on the undamaged network. 'Drivers' Delay' will have the units of hours per day. Total 'Drivers' Delay' until the transportation network has been completely restored shall be computed by integrating over all the days that a delay persists. In addition, bridge repair costs are assumed to be proportional to the bridge's replacement value, depending on its damage state and calculated accordingly.

After the Northridge earthquake the highway transportation system in Los Angeles metropolitan area revealed some system resiliency. Secondary highways and artillery streets that were an affected by the event served the purpose detouring traffic by integrating them into the expressway network that where some bridges have been damaged. Shinozuka, et al. (2000), quantified the changes in a highway’s link capabilities in terms of percentage relative to the values under intact conditions depending on the degree of the state of the link damage. Here, alternate routes are considered to exist but are expected to have lesser traffic capability. Percentage values also attempt to account for the changes resulting from the repair work, not only from the detour of traffic. This hypothetical values are presented in Table 3-3. 
Table 3-3 Change in Link Capacity and Free Flow Speed

\begin{tabular}{ccc}
\hline Damage State & $\begin{array}{c}\text { Capacity } \\
\text { Change rate }\end{array}$ & $\begin{array}{c}\text { Free Flow Speed } \\
\text { Change rate }\end{array}$ \\
\hline \hline No Damage & $100 \%$ & $100 \%$ \\
Slight & $100 \%$ & $75 \%$ \\
Moderate & $75 \%$ & $50 \%$ \\
Extensive & $50 \%$ & $50 \%$ \\
Collapse & $50 \%$ & $50 \%$ \\
\hline
\end{tabular}

The total daily travel time for all network users, in hours per day, is the product of the flow on link in passenger car unit (PCU) per day and the travel time on link in hours per PCU and is given by Equation 3-4. The product yields the total daily travel time for all network travelers on link. The total daily travel time is for every link is then added to give the total daily travel time on the entire network.

Drivers' Delay (Equation 3-5) is calculated as the difference between the total daily travel for all network travelers on the damaged network before and after a seismic event, in units of hours per day.

$$
\begin{array}{cc}
\text { Travel Time }=\sum_{\mathrm{a}} \mathrm{x}_{\mathrm{a}} \mathrm{t}_{\mathrm{a}}\left(\mathrm{x}_{\mathrm{a}}\right) & \text { Equation 3-4 } \\
\text { Drivers' Delay }=\sum_{\mathrm{a}} \mathrm{x}^{\prime}{ }_{\mathrm{a}} \mathrm{t}^{\prime}{ }_{\mathrm{a}}\left(\mathrm{x}^{\prime}{ }_{\mathrm{a}}\right)-\sum_{\mathrm{a}} \mathrm{x}_{\mathrm{a}} \mathrm{t}_{\mathrm{a}}\left(\mathrm{x}_{\mathrm{a}}\right) & \text { Equation 3-5 }
\end{array}
$$

Where $x_{\mathrm{a}}$ is the flow on link a (in PCU per day), and $\mathrm{t}_{\mathrm{a}}$ is the travel time on link a (in hours per PCU). Primed variables denote the case of the damaged network. The travel time on a link is calculated by applying a link performance function developed by the United States Bureau of Public Roads (Equation 3-7). 


$$
t_{a}=t_{a}^{0}\left[1+\alpha\left(\frac{x_{a}}{c_{a}}\right)^{\beta}\right]
$$

Equation 3-6

Where $t_{a}^{0}$ is the travel time at zero flow on link (computed as link length divided by speed limit), $C_{a}$ the practical capacity of the link $\alpha$ and $\beta$ are variable perimeters and are taken as 0.15 and 4.0. It can be seen that the empirically derived expression assumes that the travel time on a link carrying $100 \%$ of capacity is $15 \%$ greater than the free flow time.

Once the travel time delay is calculated, associated losses can be calculated as a product of 'drivers' delay' and estimated cost per hour delay. The average cost per hour delay due to traffic congestion (time and fuel) of $\$ 32.15$ is the conservative value recommended by the FHWA's Highway Economic Requirements System model. Shinozuka, et al (2000) estimate $\$ 50$ per 1 hour delay in their investigation. For this study, cost per day associated with drivers' delay will be calculated by multiplying the drivers' delay by $\$ 32.15 / \mathrm{hr}$. Although other costs may result from other negative effects of travel delays, it is reasonable to take this value as an initial and conservative estimate for the loss due to drivers' delay.

\subsubsection{Total Expected loss}

The total expected loss is then computed as the sum of the repair or replacement cost of damaged bridges and the travel time delay associated costs as is given in Equation 3-7.

$$
\text { Expected Loss }_{\text {as-built }}=\text { Repair } \text { Cost }_{\text {as-built }}+\text { Driver's Delay }_{\text {as-built }} \quad \text { Equation 3-7 }
$$




\subsection{Social Module}

The social module in this study includes estimation of downtime in days and expected number and casualty rate in level of severity from a seismic hazard.

\subsubsection{Downtime}

The amount of time it takes to repair and restore a highway bridge after earthquake damage is given by restoration curves that were developed based on a best fit to ATC-13 (1985) data for the four damage states. Figure 3-1 represents restoration curves for highway bridges. The means and standard deviations for each restoration curve are given in Table 3-4. Distributions on functionality for each restoration period based on damage state immediately after the earthquake are given in Table 3-5. However, there are apparent uncertainties in estimating downtime such as the availability of resources to make the repair or replacement, accessibility to bridge, and environmental and regional regulations (ODOT, 2013).

Table 3-4 Continuous Restoration Functions for Highways (ATC-13, 1985)

\begin{tabular}{c|c|c}
\hline \multirow{2}{*}{$\begin{array}{c}\text { Damage } \\
\text { State }\end{array}$} & \multicolumn{2}{|c}{ Highway Bridges } \\
\cline { 2 - 3 } & $\begin{array}{c}\text { Mean } \\
\text { (Days) }\end{array}$ & $\begin{array}{c}\sigma \\
\text { (Days) }\end{array}$ \\
\hline \hline Slight & 0.6 & 0.6 \\
Moderate & 2.5 & 2.7 \\
Extensive & 75.0 & 42.0 \\
Complete & 230.0 & 110.0 \\
\hline
\end{tabular}




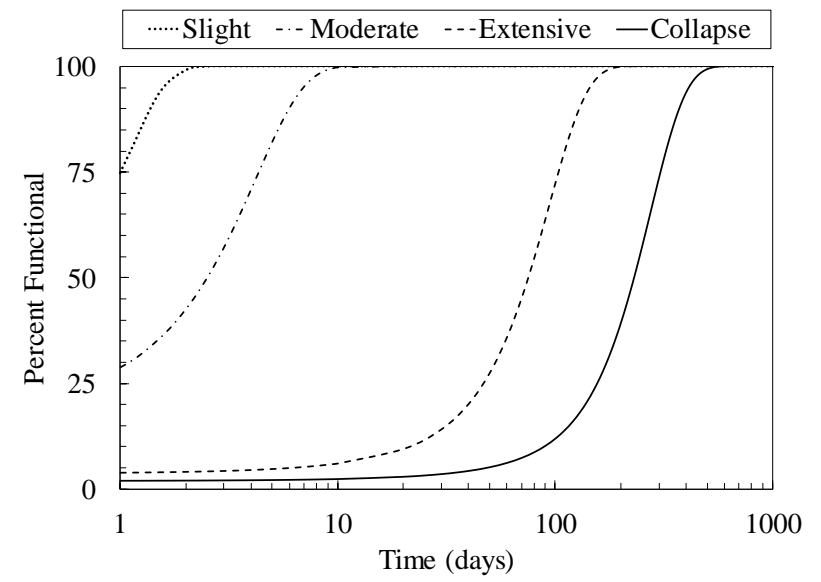

Figure 3-1 Restoration Curves for Highway Bridges (ATC-13, 1985)

Table 3-5 below represents distributions on functionality for each restoration period based on damage state immediately after the earthquake.

Table 3-5 Discrete Restoration Functions for Highways (HAZUS-MH (FEMA, 2010))

\begin{tabular}{|c|c|c|c|c|}
\hline \multirow{2}{*}{$\begin{array}{c}\text { Restoration } \\
\text { Period } \\
\text { (days) } \\
\end{array}$} & \multicolumn{4}{|c|}{ Functionality Percentage } \\
\hline & Slight & Moderate & Extensive & Complete \\
\hline 1 & 70 & 30 & 2 & 0 \\
\hline 3 & 100 & 60 & 5 & 2 \\
\hline 7 & 100 & 95 & 6 & 2 \\
\hline 30 & 100 & 100 & 15 & 4 \\
\hline 90 & 100 & 100 & 65 & 10 \\
\hline
\end{tabular}

\subsubsection{Fatalities}

Estimation of casualties from a seismic hazard is based on the assumption that there is a strong correlation between collapse of a bridge and the number and severity of casualties. This study follows the HAZUS-MH model and attempts to estimate casualties either on or under bridges that experience complete damage. The model estimates the 
number of people commuting in cars or other modes using the default relationships presented in Table 3-6. The casualty estimates were done for three times of day. These time options are earthquake striking at 2:00 a.m. (night time scenario), earthquake striking at 2:00 p.m. (day time scenario), and earthquake striking at 5:00 p.m. (commute time scenario). The number of people on and under bridges in the census tract is then computed as a factor of the number of commuters as shown in Equation 3-8. Injury categories or severity levels have been defined by various researchers (Durkin \& Thiel, 1991), (Coburn \& Spence, 1992), etc. In these studies, casualties are calculated as a percentage of the population at the census tract level which are then totaled for the study region.

Table 3-6 shows causality rates, for each severity level, that are used in the HAZUS-MH model. The data given is only for complete collapse as lack of data did not allow similar deductions for other damage states. Data was also only available for major bridges (total length $>150 \mathrm{ft}$.), continuous bridges and single span bridges. As can be seen from Table 3-7, the rates presented for both major and continuous bridges are the same. Hence, this study assumes the same rates for all other bridge types not defined in this table.

Table 3-6 Default Relationships for Estimating Population Distribution (HAZUS- MH4 Technical manual)

\begin{tabular}{c|c|c|c}
\hline \multicolumn{4}{c}{ Distribution of People in Census Tract } \\
\hline Occupancy & $2: 00$ a.m. & $2: 00$ p.m. & $5: 00$ p.m. \\
\hline \hline Commuting in cars & $0.005($ POP) & (PRFIL)0.05(POP) & $\begin{array}{c}\text { (PRFIL) [0.05(POP) }+ \\
1.0(\mathrm{COMM})]\end{array}$ \\
\hline $\begin{array}{c}\text { Commuting using } \\
\text { other modes }\end{array}$ & - & $0.50(1-\mathrm{PRFIL}) 0.05(\mathrm{POP})$ & $\begin{array}{c}0.50(1-\mathrm{PRFIL})[0.05(\mathrm{POP})+ \\
1.0(\mathrm{COMM})]\end{array}$ \\
\hline
\end{tabular}


Where:

- POP is the census tract population taken from census data

- $\mathrm{COMM}$ is the number of people commuting inferred from census data

- PRFILis a factor representing the proportion of commuters using automobiles, inferred from profile of the community ( 0.60 for dense urban, 0.80 for less dense urban or suburban, and 0.85 for rural). The default is 0.80 .

$$
\mathrm{NBRDG}=\mathrm{CDF} \times \text { Commuter Population } \quad \text { Equation 3-8 }
$$

Where:

- NBRDG Number of people on or under bridges in the census tract

- CDF Commuter Distribution Factor: Percent of commuters on or under bridges in census tract (Defaults: $\mathrm{CDF}=0.01$ day, $\mathrm{CDF}=0.01$ night and $\mathrm{CDF}=0.02$ commute time.)

Census tract population data was retrieved from the Oregon Spatial Data Library website (U.S. Census Bureau, 2018). The number of people commuting is then calculated as a factor of the population data as shown in Equation 3-9 and Equation 3-11. For this study, the working age population is defined as those aged 18 to 65 . According to the U.S. Census Bureau, Oregon's population that is under 18 and over 65 is 21.2 percent and 18 percent, respectively. The number of unemployed and people that work from home is subtracted from the working age population as approximation of the number of people commuting for the census tracts. For the year 2010, the US department of Labor: Bureau of Labor Statistics listed Oregon's unemployment rate at 10 percent. The Bureau 
also estimates an average of 10 percent of employed worked from home and 22 percent did some or all of their work at home. For this study, average value of 15 percent is used to quantify the population that worked from home.

$$
\mathrm{COMM}=\mathrm{POP}_{\text {working }_{\mathrm{age}}}-\mathrm{POP}_{\text {unemployed }}-\mathrm{POP}_{\text {work }_{\text {from }}}{ }_{\text {home }} \quad \text { Equation 3-10 }
$$

$$
\mathrm{POP}_{\text {working_age }}=\mathrm{POP}-\left(\mathrm{POP}_{65+}+\mathrm{POP}_{<18}\right)
$$

Table 3-7 Casualty Rates by Model Building Type for Complete Structural Damage (No Collapse)

(HAZUS-MH Technical manual)

\begin{tabular}{c|c|c|c|c}
\hline \multirow{2}{*}{ Building Type } & \multicolumn{4}{|c}{ Casualty Severity Level } \\
\cline { 2 - 5 } & $\begin{array}{c}1 \\
(\%)\end{array}$ & $\begin{array}{c}2 \\
(\%)\end{array}$ & $\begin{array}{c}3 \\
(\%)\end{array}$ & $\begin{array}{c}4 \\
(\%)\end{array}$ \\
\hline \hline Major Bridge & $17 \%$ & $20 \%$ & $37 \%$ & $7 \%$ \\
\hline Continuous Bridge & $17 \%$ & $20 \%$ & $37 \%$ & $7 \%$ \\
\hline Single Span Bridge & $5 \%$ & $25 \%$ & $20 \%$ & $5 \%$ \\
\hline
\end{tabular}

Table 3-8 Injury Classification Scale (HAZUS-MH Technical manual)

\begin{tabular}{c|l}
\hline Injury Severity Level & Injury Description \\
\hline Severity 1 & Injuries requiring basic medical aid without requiring hospitalization \\
\hline Severity 2 & $\begin{array}{l}\text { Injuries requiring a greater degree of medical care and hospitalization, but } \\
\text { not expected to progress to a life threatening status }\end{array}$ \\
\hline Severity 3 & $\begin{array}{l}\text { Injuries that pose an immediate life threatening condition if not treated } \\
\text { adequately and expeditiously. The majority of these injuries are the result } \\
\text { of structural collapse and subsequent entrapment or impairment of the } \\
\text { occupants. }\end{array}$ \\
\hline Severity 4 & Instantaneously killed or mortally injured \\
\hline
\end{tabular}

Various researchers have tried to quantify Value of Statistical Life (VSL). VSL is an economic value used to quantify the benefit of avoiding a fatality, also referred to as value of preventing fatality. There is no doubt that life is priceless and putting a price 
tag may seem cruel. However, it is necessary to do so for comparative purposes to quantify the significance of human losses to other direct and indirect losses due to repair and replacement of damaged bridges and cost associated with travel time delay and downtime. In statistical terms, VSL is the cost of reducing the number of deaths by one. There are different types of approaches various groups use to evaluate VSL. The U.S. Department of Transportation (2007) has suggested VSL $=\$ 5.8$ million. However, as of August of 2016, those values have been updated to $\$ 9.6$ million (U. S. Department of Transportation, 2016). Based on the above, a value of VSL $=\$ 9.6$ million shall be used in this work.

\subsection{Scenario Earthquakes}

The earthquake hazard considered was ground motion only. Possible liquefaction, landslide and other hazards that can result due to an earthquake were not included in the scope of this study. The earthquake scenarios that are considered for this study are subduction zone earthquakes and crustal earthquakes. The U. S. Geological Survey (USGS) developed ShakeMap as a tool to produce recorded and predicted strong ground motions. A ShakeMap earthquake scenario is a predictive ShakeMap with an assumed magnitude and location, and, optionally, specified fault geometry. These scenario earthquakes can be used to study the exposure of structures to specified potential earthquakes and allow in the performance of seismic risk assessment to assess the vulnerability of current state of infrastructure.

Though no earthquakes have been recorded on the Cascadia subduction zone during Oregon's short 150-year historical record, numerous studies have found 
widespread evidence that very large earthquakes have occurred, most recently about 300 years ago, in January 1700 (e.g., Atwater, 1987; Yamaguchi and others, 1997). The best available evidence and observations indicate that these earthquakes occur on average about every 500 years. Hence, it is important to make an analysis of a scenario CSZ earthquake to make a reasonable prediction of the effects of the assumed earthquake. This knowledge of the potential damage will allow for planning and preparedness purposes. Crustal earthquakes occur in the North American plate at relatively shallow depths of 10$20 \mathrm{~km}$ (6-12 mi) below the surface. The 1993 magnitude 5.6 earthquake at Scotts Mills, Oregon (Madin and others, 1993) and the 1993 magnitude 5.9 and 6.0 Klamath Falls, Oregon, main shocks (Wiley and others, 1993) are examples of crustal earthquakes that have occurred in Oregon. Consequently, Crustal Earthquake Scenarios are also examined for the Oregon model. For this study, nine ShakeMap scenarios developed by USGS were utilized. Of the nine scenarios, five are Cascadia subduction zone earthquake scenarios. These selected scenarios are shown in Figure 3-2.

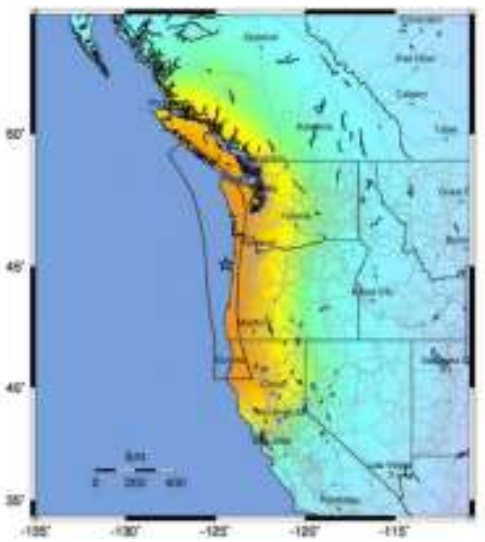

(a)

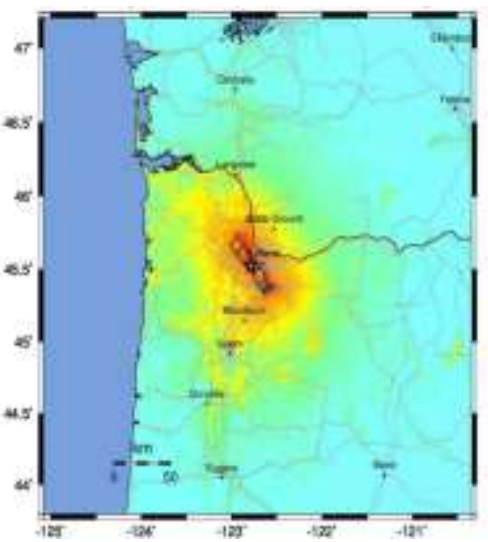

(b)

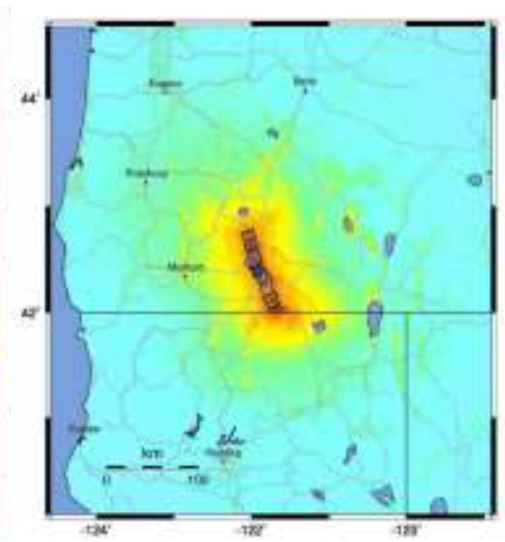

(c) 

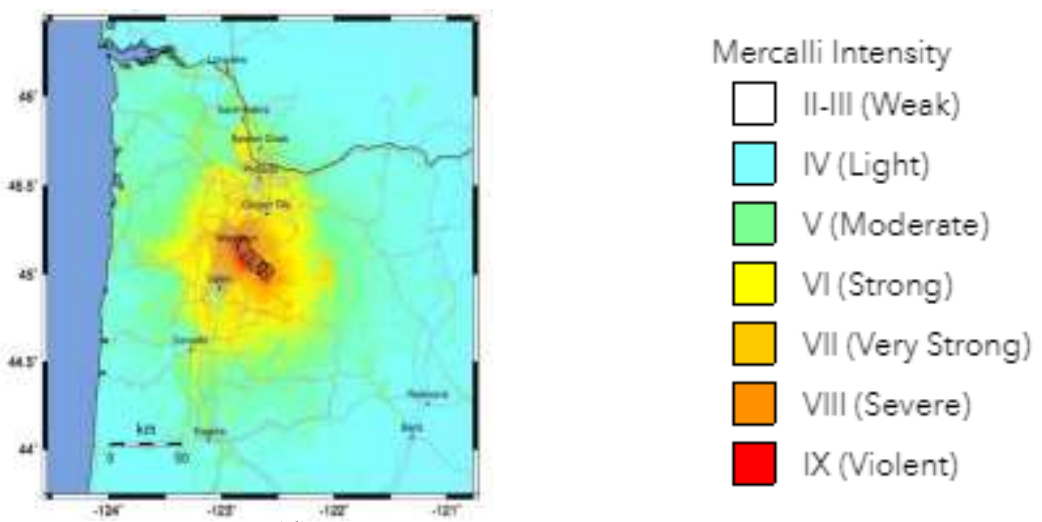

(d)

Figure 3-2 Selected ShakeMap Scenarios Developed (USGS): (a) CSZ M9.34 (b) Portland Hills M7.0 (c)

Klamath Falls M7.36, and (d) Mt. Angels M6.8

\subsection{Seismic Vulnerability Assessment Model}

The focus of the seismic vulnerability assessment has been on bridges laying on or crossing over Oregon highway routes in the area defined by Figure 3-3. The area includes all highway routes lying inside or west of the I-5 corridor, highway routes in the Portland area, the entire length of US-101 and a partial I-84 Columbia River Highway. The bridge data collected includes bridges up to the year 2010. The objective of the vulnerability assessment is to find the expected damage and/or loss.

National Highway Planning Network (NHPN) and Highway Performance Monitoring System (HPMS) are nationally available transportation databases that model the spatial configuration and attributes of the roadways in the study area. The databases are assembled by the individual states and distributed by FHWA. Furthermore, roadway systems are divided into a set of sub regions called Traffic Analysis Zones (TAZs) to monitor user trip demands on the roadway system. The local and state governments do this subdivision. TAZs are small areas approximately the size of a census tract. Origin- 
Destination (O-D) data estimates the location of travel origins and destinations and the corresponding number of trips from and to all the different TAZs in the region, and is compiled by local metropolitan planning organizations from periodic public surveys. The transportation data was located in the previous study done by Dusicka et al. (2007).

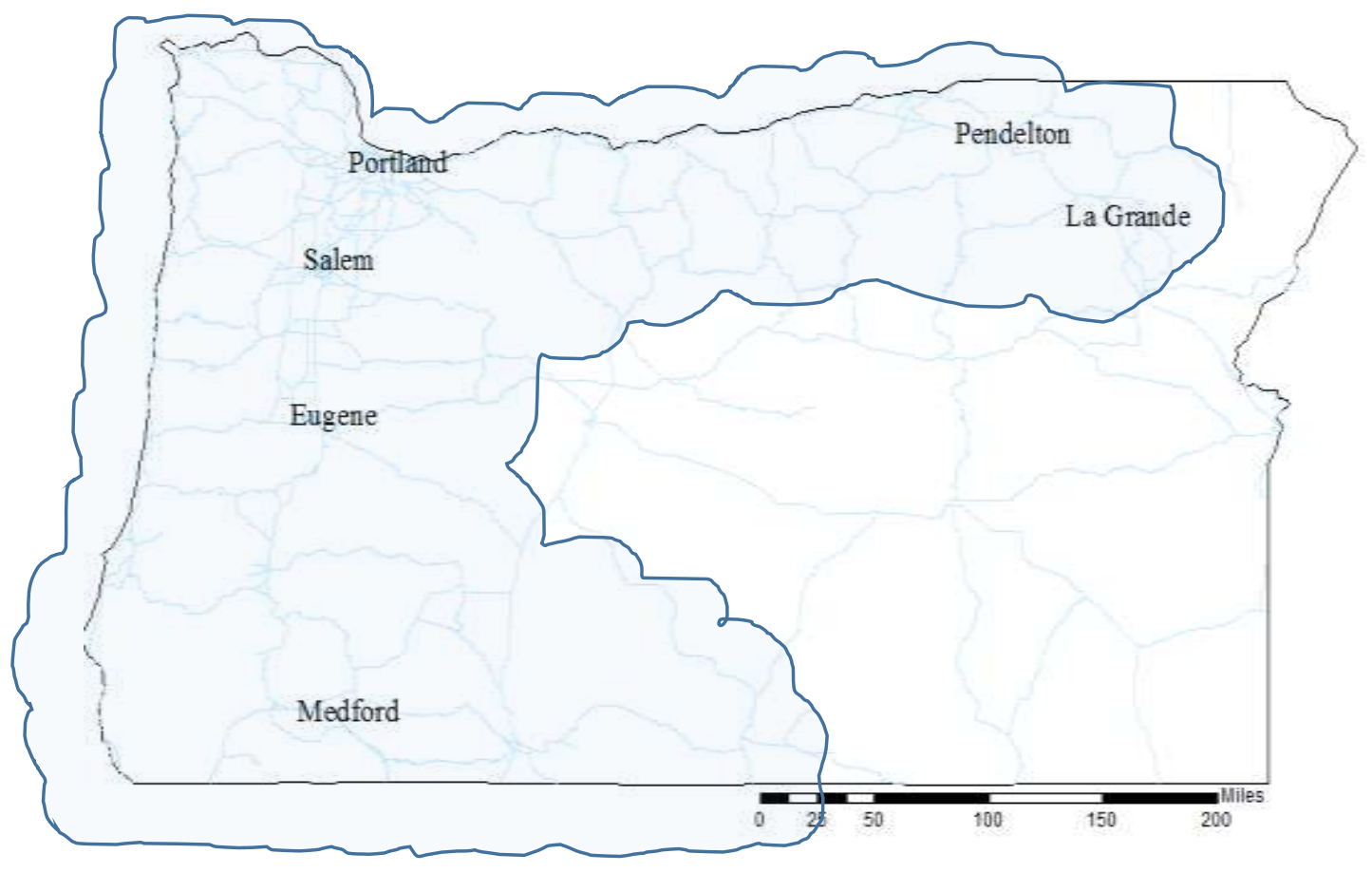

Figure 3-3 Study Area

\subsection{Steps in Damage Algorithm for Bridges}

\subsubsection{Input Requirements}

Structural attribute data for each bridge in the roadway system were obtained from the Federal Highway Administration's National Bridge Inventory (NBI) program database where bridges nationwide are recorded. The structural attributes that are obtained from the NBI database include information such as bridge type, number of spans, total length of maximum span, year of construction, skew angle, deck width, 
structure length, minimum vertical clearance, etc. These parameters are used to infer bridge damage-state fragilities.

\subsubsection{Ground Motion Data}

The demand ground motions (spectral accelerations at periods of $0.3 \mathrm{sec}$. and 1.0 sec.) at each bridge site are obtained by using ArcMap and overlaying the ShakeMap scenario earthquake maps over the bridges and reading corresponding spectral acceleration and peak ground acceleration data.

\subsubsection{Damage Functions}

The fragility curves available for generalized classes of bridges in HAZUS-MH based on the work of Basoz and Mander (1999) are given in Table 0-3 are used to obtain the median spectral acceleration capacity of each bridge type at a period of $1.0 \mathrm{sec}$. These values are for a "standard bridge" type and will be modified to convert to the actual bridge, where the effect of skew and three-dimensional deck-arching membrane action are accounted for. The new medians along with a dispersion $\beta=0.6$ were then used to evaluate the ground shaking related damage state probabilities. The functionality of the bridges is then specified in damage state levels.

\subsubsection{Network Analysis}

To create the Oregon network model and define the transportation network and the associated traffic flow, six categories of data were collected, analyzed and modified. These data were National Highway Planning Network Data (NHPN), Highway Performance Monitoring System Database (HPMS), National Bridge Inventory Database 
(NBI), Supplemental Geotechnical Data (collected by the user), Traffic Analysis Zone Map of the region (TAZ map), and Origin-Destination Trip Data (O-D Matrices). The bridges are the vulnerable links within the network and when damaged, change in the traffic demand is placed onto the system. The damage state of each bridge determines the link capacity, where the worst state of the bridge damage in the link determines the state of the link damage.

\subsection{Seismic Vulnerability Assessment Results}

Seismic risk assessment estimations of expected damage, expected economic loss, and fatalities were computed for each of the nice scenario earthquakes, are results are presented in the following section.

\subsubsection{Cascadia Subduction Zone Earthquake near Northern Oregon (M8.3)}

In this earthquake model, an earthquake scenario of magnitude 8.3 at the Cascadia Subduction Zone near northern Oregon produced no complete collapses, 1 extensive, 17 moderate and 34 slight damage states. 65 percent of the damaged bridges are located on US-101. The losses evaluated were $\$ 76$ million for bridge repair and replacement and $\$ 6$ million per day in travel time delay related losses. Figure 3-4 shows the spatial distribution of damaged bridges and PGA. Since there were no complete collapse cases, no fatality is computed for this scenario. 

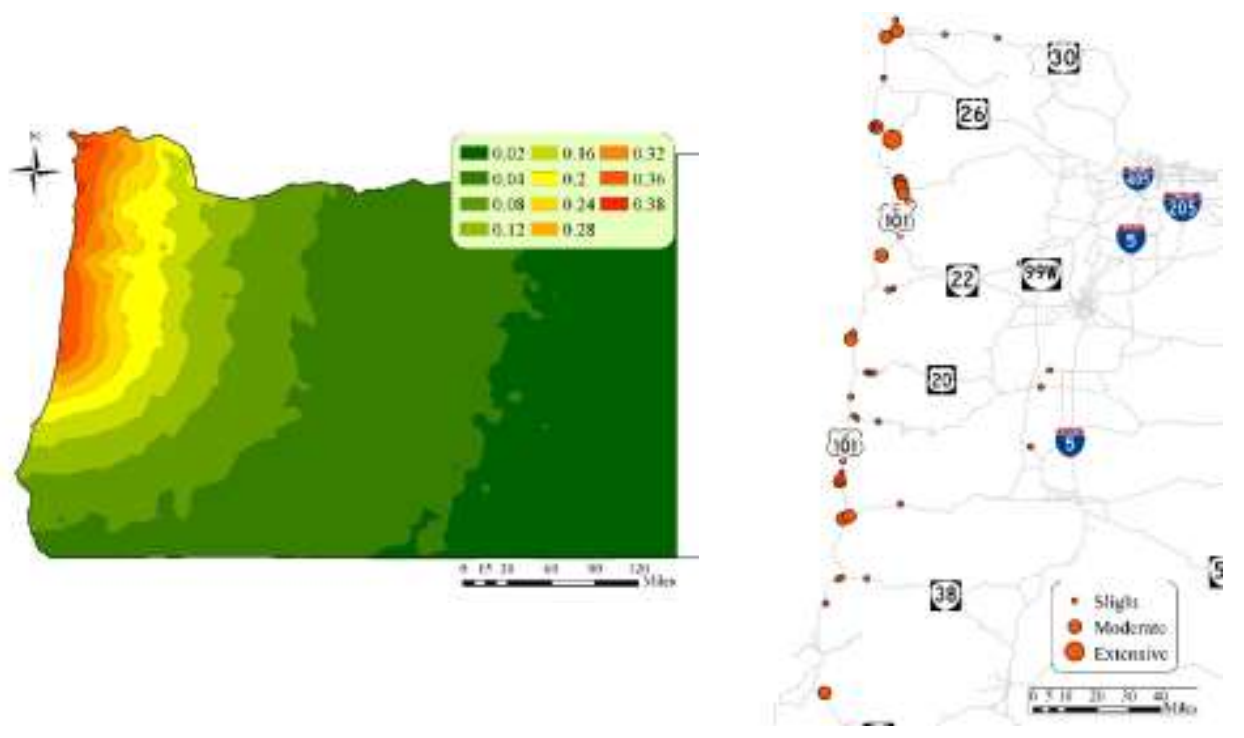

Figure 3-4 CSZ M8.3 North Scenario PGA Distribution and Component Damage States

\subsubsection{Cascadia Subduction Zone Earthquake near Southern Oregon (M8.3)}

For the magnitude 8.3 Cascadia Subduction Zone earthquake scenario near Southern Oregon, the estimates were 1 complete collapse, 12 extensive, 11 moderate and 22 slight damage states. Figure 3-5 shows the distribution of damages bridges and the PGA for the southwestern part of Oregon. 45 percent of the damages were on US-101 and 35 percent were on OR-40. The losses evaluated were $\$ 168$ million for bridge repair and replacement and $\$ 17$ million per day in travel time related losses. The number of casualties estimated is 52 . These casualties vary in severity and 5 commuters are expected to be instantaneously killed or mortally injured. The amount it would cost to prevent these life losses is estimated to be around $\$ 50$ million. 

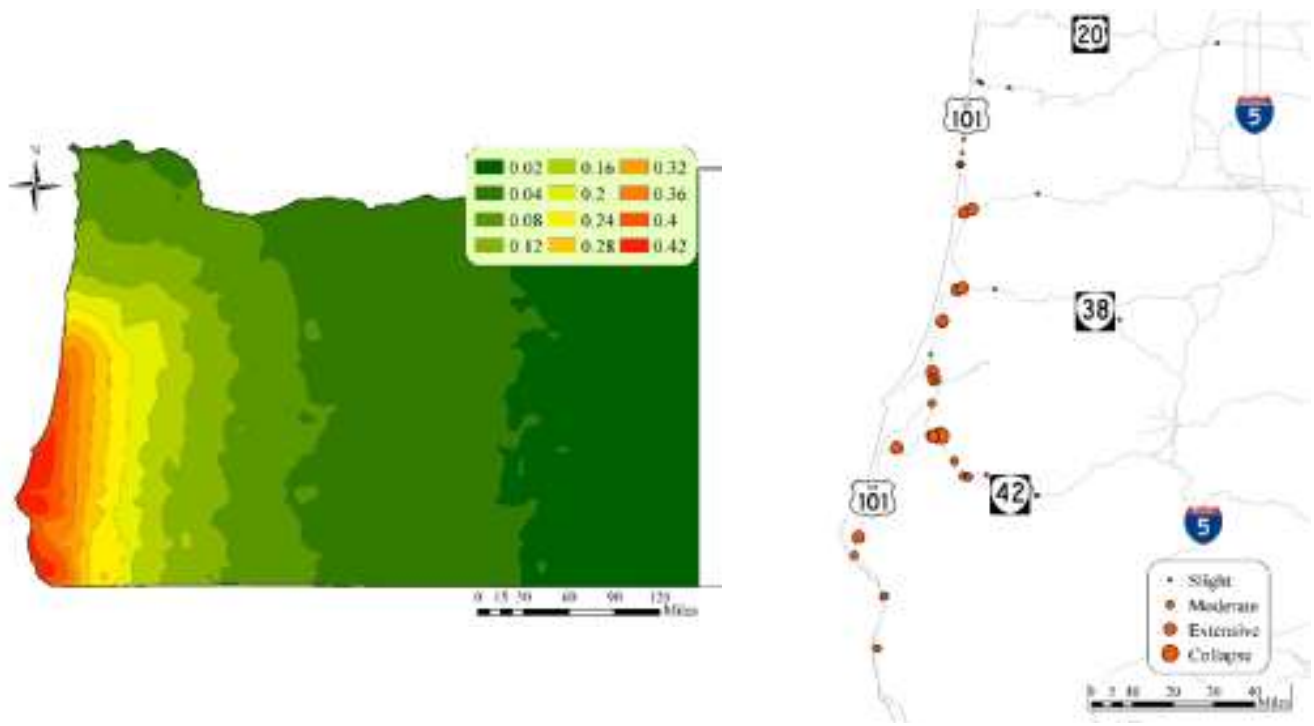

Figure 3-5 CSZ M8.3 South Scenario PGA Distribution and Component Damage States

\subsubsection{Cascadia Megathrust - Full length CSZ}

Three full-length Cascadia megathrust earthquake scenarios were also those applied as ground motion sources for this study. Two of the scenarios have magnitude 9.0 and the third one has a magnitude of 9.34 .

For the first earthquake scenario of magnitude 9.0 at the Cascadia Subduction Zone, the PGA distribution is shown Figure 3-6. The analysis resulted in 1 complete collapse, 24 extensive, 33 moderate and 108 slight damage states. Figure 3-6 shows a map of component damage states for the western part of Oregon. Similar to the Cascadia subduction scenario near southern Oregon, majority of the damage is on US-101 at 34 percent and approximately 20 percent of the damaged bridges lay on OR-42. The losses calculated were $\$ 412$ million for bridge repair and replacement and it will also cost $\$ 9.31$ million per day in travel time related losses. The number of casualties in this case is also estimated to be 52 . These casualties vary in severity and 5 commuters are expected to be 
instantaneously killed or mortally injured. The amount it would cost to prevent these life losses is estimated to be around \$194 million.
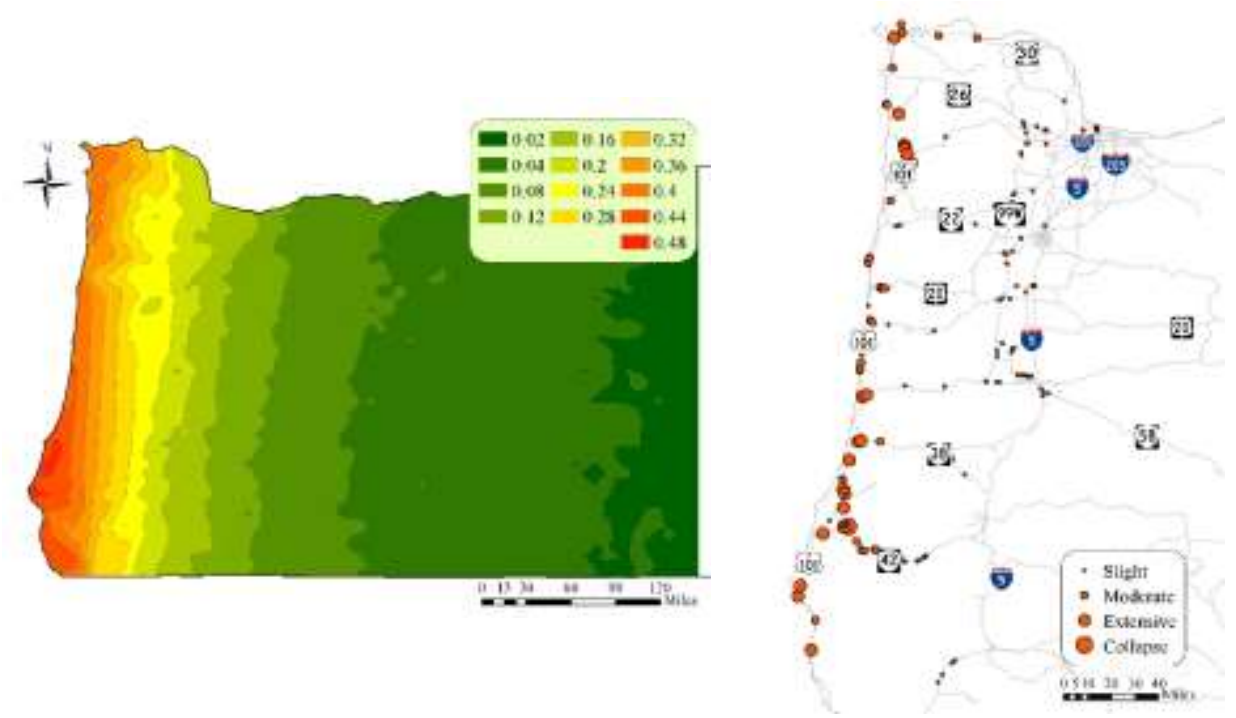

Figure 3-6 CSZ 9.0 - Scenario (1) PGA Distribution and Component Damage States

The second earthquake scenario of magnitude 9.0 at the Cascadia Subduction Zone gave similar outcomes as the first CSZ M9.0 scenario where majority of the damage was localized to US-101 and OR-42 at 27 and 15 percent of total damage, respectively. Figure 3-6 shows a map of component damage states for the western part of Oregon. The level of damages were computed at 1 complete collapse, 27 extensive, 39 moderate and 117 slight damage. The associated losses were calculated as \$413 million for bridge repair and replacement and $\$ 53$ million per day in travel time related losses. The number of casualties and amount it would cost to prevent life losses is the same as the previous CSZ full length event. 

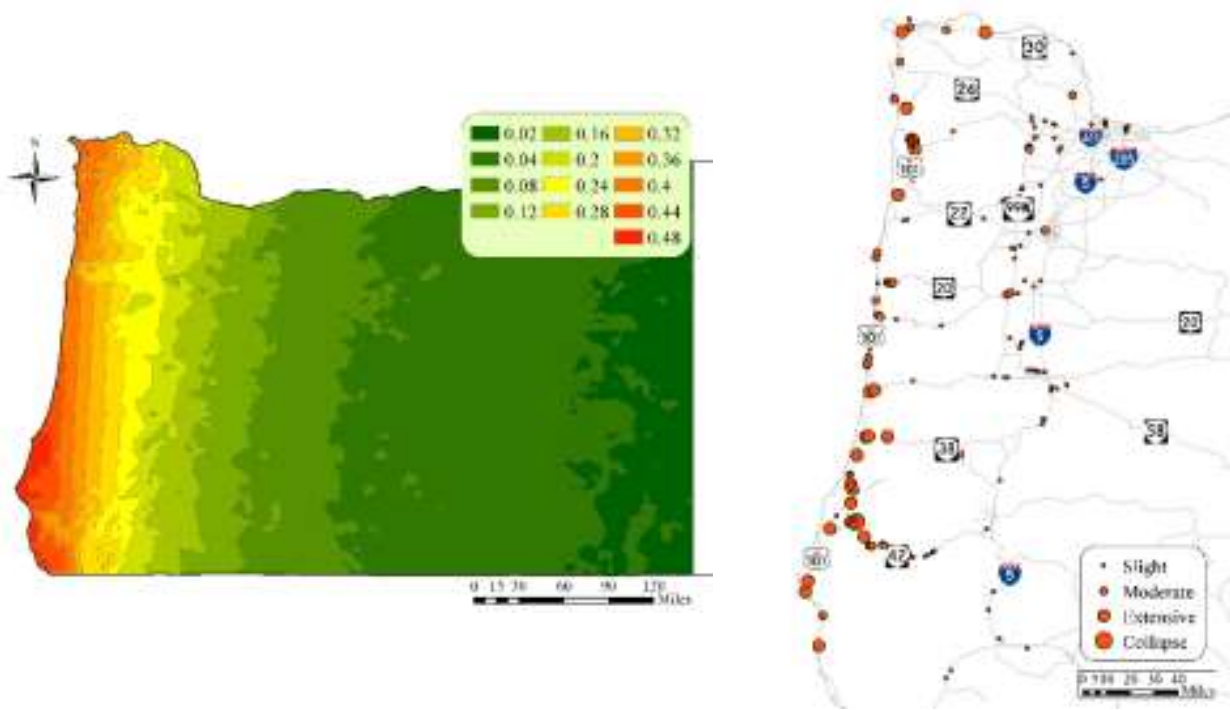

Figure 3-7 CSZ 9.0 - Scenario (2) PGA Distribution and Component Damage States

The third full-length Cascadia Subduction Zone earthquake scenario with magnitude 9.34, as expected, resulted in more damages than all the scenarios. The assessment estimates 30 complete collapses, 213 extensive, 114 moderate and 157 slight damage states. Figure 3-6 shows a map of component damage states for the western part of Oregon. Of the bridges damages, 21 percent of the bridges lay on I-5 and another 20 percent on lay US-101. From the damaged bridges, losses were calculated and estimate $\$ 3,582$ million for bridge repair and replacement and a cost of $\$ 573$ million per day in travel time related losses. For this assessment, the number of casualties estimated around 1000. These casualties vary in severity and 600 of which are expected to be severity level 3 and 4 . These commuters are expected to sustain life threatening injuries or are instantaneously killed or mortally injured. The amount it would cost to prevent these life losses is estimated at around $\$ 1$ billion. 

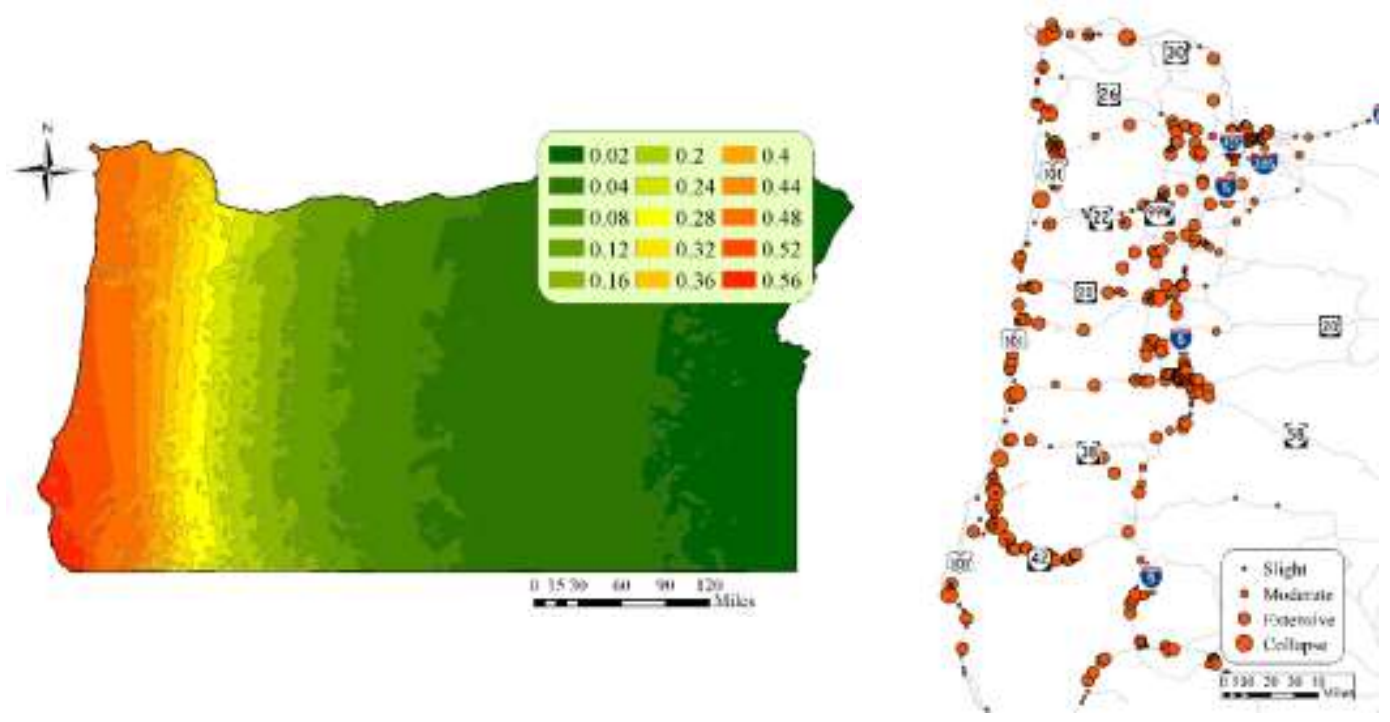

Figure 3-8 CSZ 9.34 Scenario PGA Distribution and Component Damage States

\subsubsection{Crustal Earthquake Scenario - Portland Hills Fault (M7.0)}

The Portland Hills fault zone lies just east of Washington County and runs directly under downtown Portland. A large-magnitude earthquake in the Portland Hills fault zone would cause strong ground shaking and damages in Washington County. Hence, two of the scenarios selected for this study were for Portland hills earthquake scenarios of magnitude 7.0.

For the first Portland Hills scenario, it was estimated that there will be no complete collapses, 2 extensive, 7 moderate and 46 slight bridge damage states. Approximately 50 percent of the damaged bridges are on I-5 in the Portland metro and valley zone. 15 percent of the bridges that are damaged are located on I-405. The losses were calculated as $\$ 216$ million for bridge repair and replacement and $\$ 8$ million per day in travel time related losses. Since there were not bridge collapses, there are no casualties. Figure 3-9 shows a map of the component damage states in the Portland Metro Area 


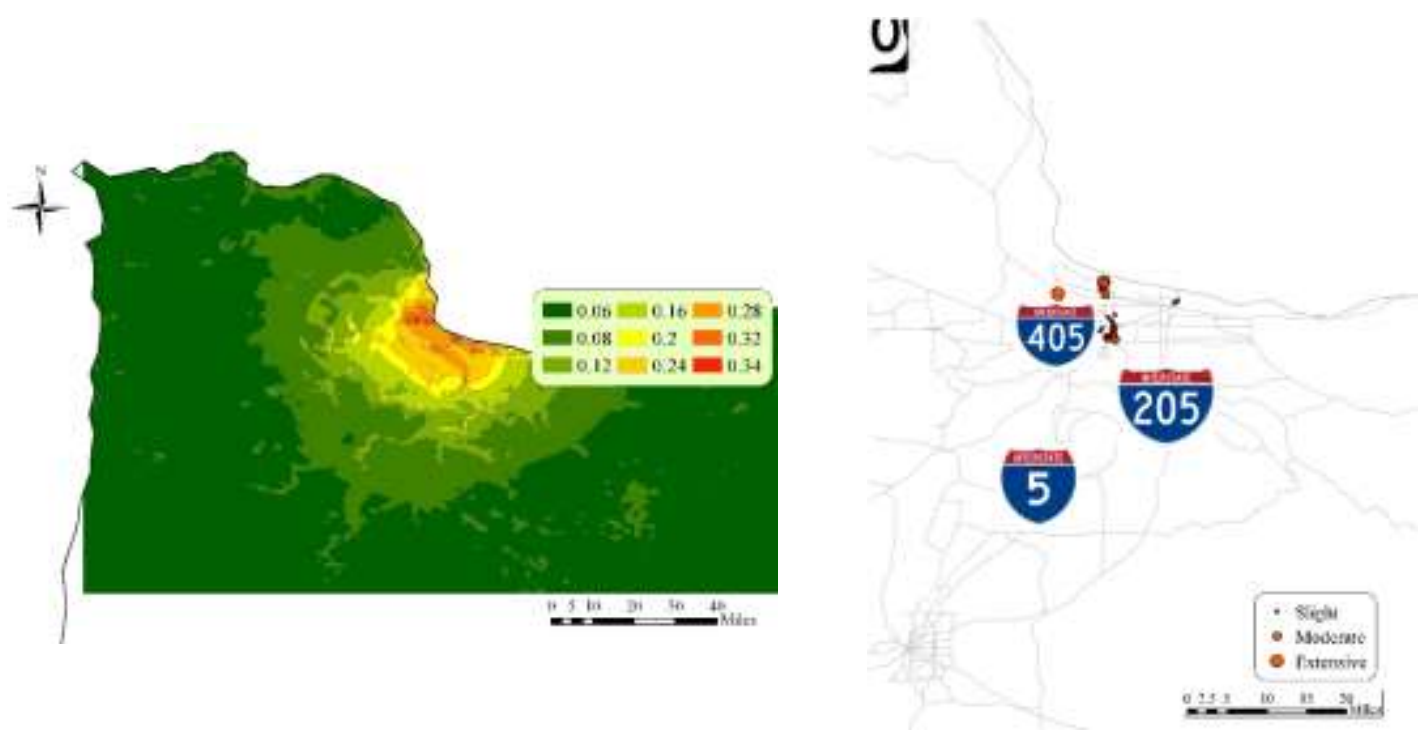

Figure 3-9 Portland Hills Fault - Scenario (1) PGA Distribution and Component Damage States

For the second earthquake scenario of magnitude 7.0 in the Portland Metro Area has larger coverage as can be seen in the PGA distribution map on Figure 3-10. The number and spread of damaged bridges is also larger. The assessment estimated 55 complete collapses, 67 extensive, 12 moderate and 119 slight bridge damage states. Most of the damaged bridges are located on I-5, I-205 and I-405 making up 23, 14 and 13 percent of the total number of damaged bridges, respectively. The economic impact of these damages was calculated to be $\$ 3,863$ million for bridge repair and replacement and $\$ 51$ million per day in travel time related losses. The number of casualties estimated is over 1200. These casualties vary in severity and over 60 percent of these casualties are likely to sustain injuries that pose an immediate life threatening condition if not treated adequately and expeditiously or are instantaneously killed or mortally injured. Due to the large number of bridges that are expected to collapse in such an event, the amount it would cost to prevent these life losses is estimated at around $\$ 1$ billion. 

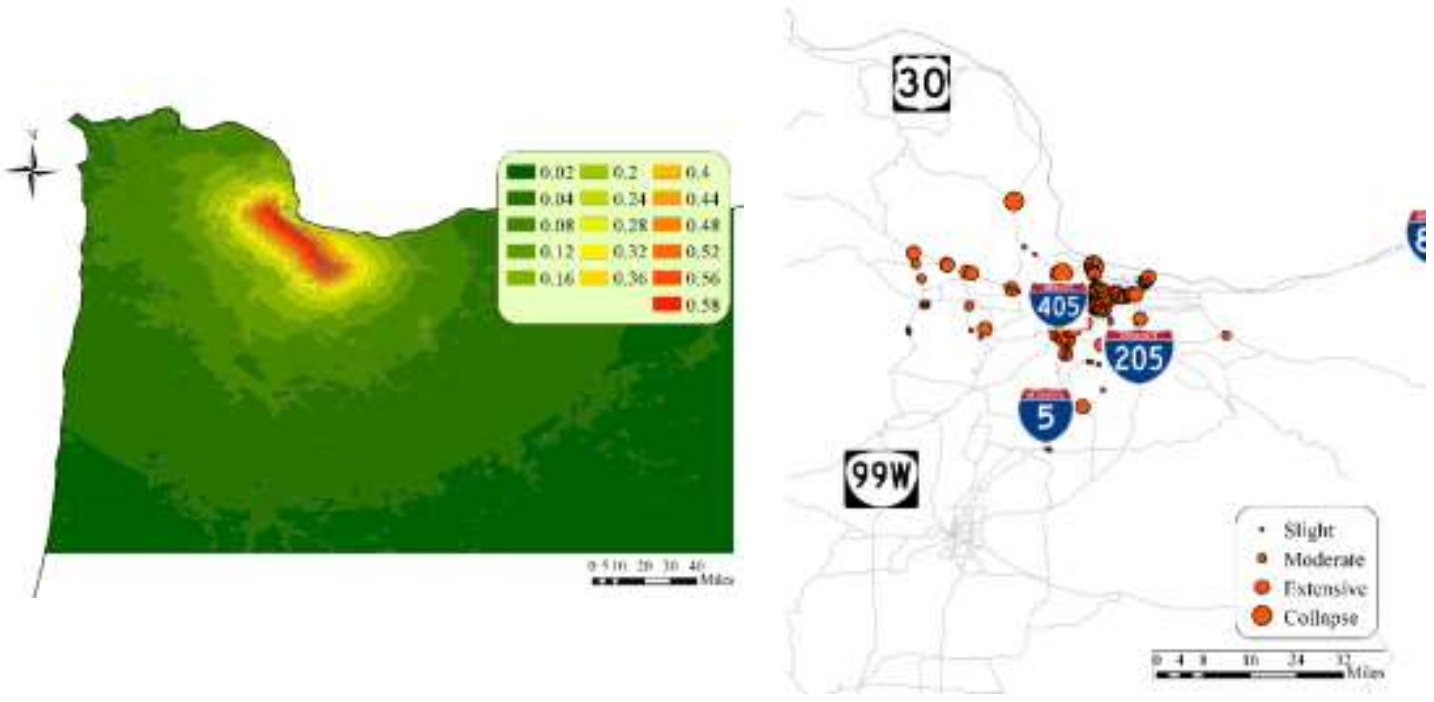

Figure 3-10 Portland Hills Fault - Scenario (2) PGA Distribution and Component Damage States

\subsubsection{Crustal Earthquake Scenario - Klamath Graben Fault System (M7.36)}

The Klamath graben fault system is a group of normal faults that form a complex graben system that confines the Klamath Lake basin. The Klamath graben fault system is divided into the West Klamath Lake section, the East Klamath Lake section, and the south Klamath Lake section. The scenario in this study is the west section. A magnitude 7.36 scenario earthquake around Klamath fault resulted in 4 complete collapses, 8 extensive, 2 moderate and 13 slight damage states. Figure 311 shows a map of the component damage states in the Klamath Faults Area. The losses estimated were \$160 million for bridge repair and replacement and $\$ 2$ million per day in in travel time related losses. For this assessment, the number of casualties estimated is 108 . These casualties vary in severity and 11 of which being severity level 4 where commuters are instantaneously killed or mortally injured. The amount it would cost to prevent these life losses is estimated at around $\$ 100$ million. 

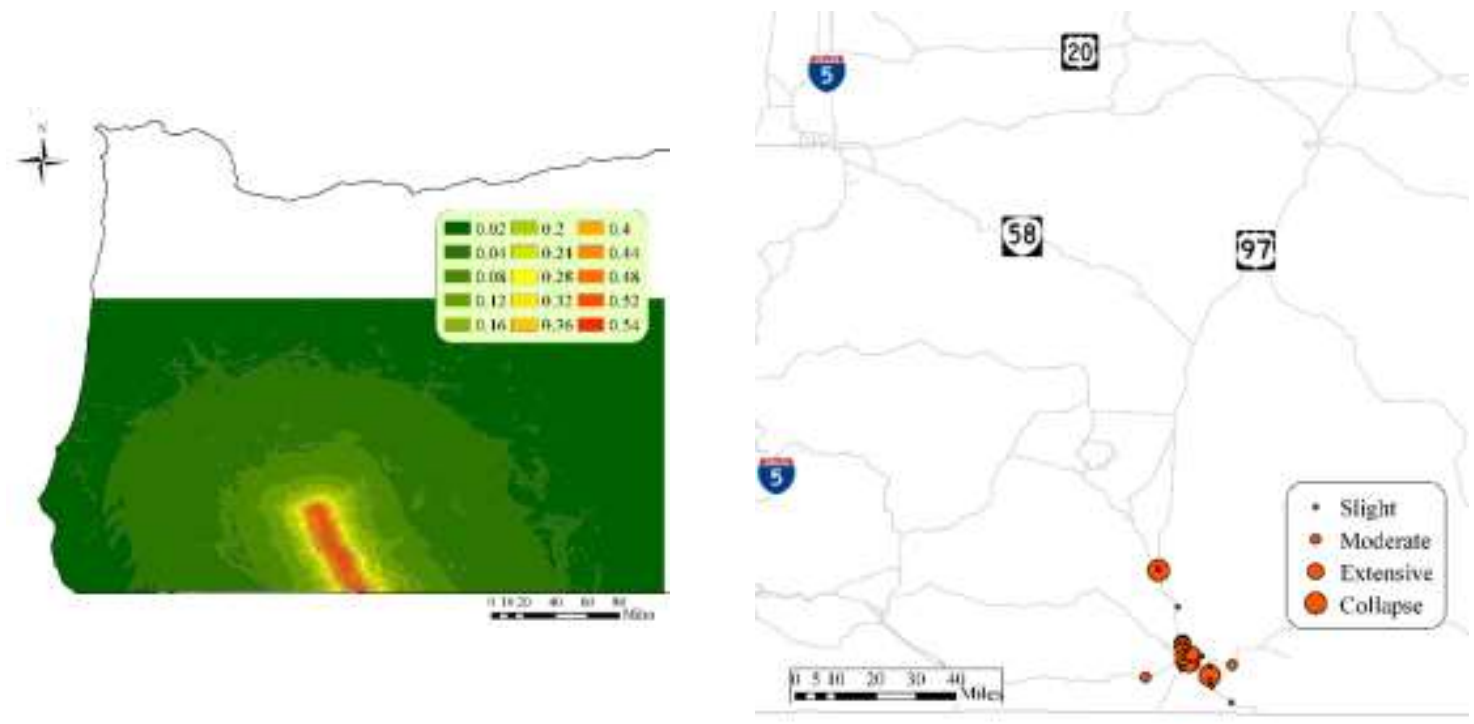

Figure 3-11 Klamath Fault Scenario PGA Distribution and Component Damage States

\subsubsection{Crustal Earthquake Scenario - Mount Angel Fault (M6.80)}

Mount Angel fault zone is one of the three active zones close to the Portland metro Area and is located in the northern Willamette Valley. The 1993 magnitude 5.6 Scotts Mills earthquake was one of the most damaging earthquakes in Oregon's history and originated from the Mount Angel fault zone. For that reason, the last crustal earthquake scenario selected was Mt Angels fault magnitude 6.80.

The assessment resulted in 3 complete collapses, 3 extensive, 5 moderate and 14 slight damage states. Figure 312 shows a map of the component damage states in the Mt. Angels area. A third of the bridges that were damaged are located on I-5. The corresponding losses estimated were $\$ 76$ million for bridge repair and replacement and $\$ 68$ million per day in in travel time related losses. The number of casualties estimated is 
102. These casualties vary in severity and 10 of which being severity level 4 where commuters are instantaneously killed or mortally injured. The amount it would cost to prevent these life losses is estimated at $\$ 97$ million.

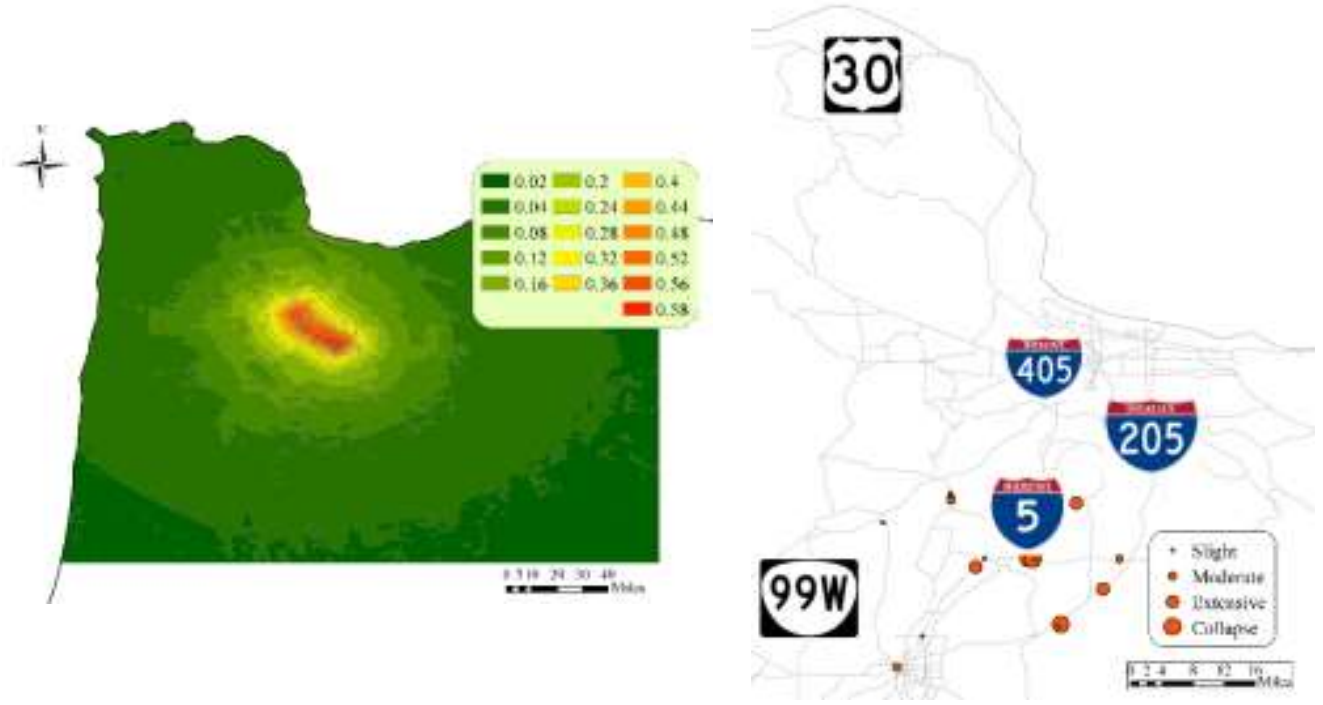

Figure 3-12 Mt Angels Fault Scenario PGA Distribution and Component Damage States

\subsection{Summary}

Table 3-9 and Table 3-10 present a summary of the seismic hazard analysis results in terms of numbers of damaged bridges and the associated economic losses. The sources of economic loss were bridge repair or replacement cost and losses due to seismic hazard induced travel time delays. For the social matrix, causality count per severity level for the nine earthquake scenario ground motions are given in Table 3-10 and the corresponding cost associated with fatality is given in Table 3-11. The downtime, which is the amount of time it takes to repair and restore a highway bridge, is computed according to the restoration functions that are given in Table 3-4 and Figure 3-1. For each 
bridge with a slight, moderate, extensive and collapse damage states, the time it takes, on average, to restore each bridge is 0.6 days, 2.5 days, 72 days and 230 days, respectively.

Table 3-9 Summary of Damage States and Loss Estimates

\begin{tabular}{l|c|c|c|c|c|c}
\hline \multirow{2}{*}{ EQ Scenario } & \multicolumn{4}{|c|}{$\begin{array}{c}\text { Damage State } \\
\text { (No of Bridges) }\end{array}$} & \multicolumn{2}{c}{$\begin{array}{c}\text { Economic Loss } \\
\text { (\$ in millions) }\end{array}$} \\
\cline { 2 - 7 } & & & & & $\begin{array}{c}\text { Repair or } \\
\text { Replacement } \\
\text { Cost }\end{array}$ & $\begin{array}{c}\text { Travel Time } \\
\text { Delay } \\
\text { Cost/Day }\end{array}$ \\
\hline \hline CSZ M8 North & 34 & 17 & 29 & 1 & 881 & 6 \\
CSZ M8 South & 22 & 11 & 12 & 1 & 168 & 17 \\
CSZ M9(a) & 108 & 33 & 24 & 1 & 412 & 194 \\
CSZ M9(b) & 117 & 39 & 27 & 1 & 913 & 53 \\
CSZ M9.34 & 157 & 114 & 213 & 30 & 3,582 & 573 \\
Portland Hills M7(a) & 46 & 7 & 2 & 0 & 216 & 8 \\
Portland Hills M7(b) & 119 & 12 & 67 & 55 & 3,863 & 51 \\
Klamath Falls M7.36 & 13 & 2 & 8 & 4 & 160 & 2 \\
Mt. Angeles fault M6.8 & 14 & 5 & 3 & 3 & 76 & 118 \\
\hline
\end{tabular}

Table 3-10 Casualty Count per Severity Level

\begin{tabular}{|c|c|c|c|c|c|c|c|c|c|c|c|c|}
\hline \multirow{3}{*}{ EQ Scenario } & \multicolumn{4}{|c|}{ 2:00 AM } & \multicolumn{4}{|c|}{ 2:00 PM } & \multicolumn{4}{|c|}{ 5:00 PM } \\
\hline & \multicolumn{4}{|c|}{ Severity Level } & \multicolumn{4}{|c|}{ Severity Level } & \multicolumn{4}{|c|}{ Severity Level } \\
\hline & 1 & 2 & 3 & 4 & 1 & 2 & 3 & 4 & 1 & 2 & 3 & 4 \\
\hline CSZ M8N & 0 & 0 & 0 & 0 & 0 & 0 & 0 & 0 & 0 & 0 & 0 & 0 \\
\hline CSZ M8S & 0 & 0 & 0 & 0 & 1 & 1 & 1 & 0 & 12 & 15 & 27 & 5 \\
\hline CSZ M9(a) & 0 & 0 & 0 & 0 & 1 & 1 & 1 & 0 & 12 & 15 & 27 & 5 \\
\hline CSZ M9(b) & 0 & 0 & 0 & 0 & 1 & 1 & 1 & 0 & 12 & 15 & 27 & 5 \\
\hline CSZ M9.3 & 1 & 2 & 3 & 1 & 12 & 14 & 25 & 5 & 256 & 301 & 557 & 105 \\
\hline Portland Hills M7(a) & 0 & 0 & 0 & 0 & 0 & 0 & 0 & 0 & 0 & 0 & 0 & 0 \\
\hline Portland Hills M7(b) & 2 & 2 & 3 & 1 & 14 & 16 & 29 & 6 & 299 & 352 & 650 & 123 \\
\hline Klamath falls M7.36 & 0 & 0 & 0 & 0 & 1 & 1 & 3 & 0 & 26 & 31 & 57 & 11 \\
\hline Mt. Angeles M6.8 & 0 & 0 & 0 & 0 & 1 & 1 & 2 & 0 & 24 & 29 & 53 & 10 \\
\hline
\end{tabular}


Table 3-11 Cost Associated with Fatality, VSL (in Millions)

\begin{tabular}{l|ll|lr|rr}
\hline \multirow{2}{*}{ EQ Scenario } & \multicolumn{5}{|c}{ Time of Day } \\
\cline { 2 - 7 } & $2: 00 \mathrm{AM}$ & \multicolumn{2}{|c}{$2: 00 \mathrm{PM}$} & $5: 00 \mathrm{PM}$ \\
\hline \hline CSZ M8N & $\$$ & - & $\$$ & - & $\$$ & - \\
CSZ M8S & $\$$ & 0 & $\$$ & 2 & $\$$ & 49 \\
CSZ M9(a) & $\$$ & 0 & $\$$ & 2 & $\$$ & 49 \\
CSZ M9(b) & $\$$ & 0 & $\$$ & 2 & $\$$ & 49 \\
CSZ M9.3 & $\$$ & 5 & $\$$ & 46 & $\$$ & 1,012 \\
Portland Hills M7(a) & $\$$ & - & $\$$ & - & $\$$ & - \\
Portland Hills M7(b) & $\$$ & 6 & $\$$ & 53 & $\$$ & 1,181 \\
Klamath falls M7.36 & $\$$ & 1 & $\$$ & 5 & $\$$ & 103 \\
Mt. Angeles M6.8 & $\$$ & 0 & $\$$ & 4 & $\$$ & 97 \\
\hline
\end{tabular}

Damage states of bridges are computed by first determining median values of ground motion for each bridge and comparing each bridge's median spectral acceleration capacity that leads to the onset of each damage state. However, the actual levels of ground shaking that affect the seismic performance of the bridges may not be correctly estimated since the actual levels of ground shaking of an earthquake cannot be known without actually recording the motion with strong motion accelerators. For these reasons, the aggregate response over the route should be examined and is more informative. The information that can be gathered from the above outcomes is that the seismic risk assessment results highlight the vulnerability of Oregon's highway system. Therefore, in order to minimize losses and disruptions caused by a seismic event, mitigation steps should be taken to address the vulnerability.

A bridge seismic retrofit program with the objectives of minimizing risks of bridge collapse is needed. Oregon Department of Transportation (ODOT) recognizes that it is impossible to retrofit all vulnerable bridges in the near future. Therefore, a 
prioritization methodology is needed to minimize loss of life and disruption to traffic. The following chapters will focus on retrofit options and recommendation of a retrofit prioritization methodology. 


\section{CHAPTER 4}

\section{SEISMIC RETROFITTING FOR SQUARE REINFORCED CONCRETE BRIDGE COLUMNS}

\subsection{General}

Structural failure in seismic events can be prevented if the structure is designed to achieve satisfactory deformation capacity. The deformation capacity of existing bridges can be improved by modifying substructure elements and connections. Retrofitting of bridge column is one way to increase the overall ductility of the bridge. Ductile structures dissipate more energy than brittle structures so they can be designed for lower lateral loads. Retrofit options such as reinforced concrete jacketing, steel jacketing, active confinement by prestressing wire, and composite fiber/epoxy jacketing are used to upgrade the performance of seismically vulnerable bridge columns. In this chapter, the process in the selection of a representative bridge for Oregon and the retrofit measure selected will be discussed.

A study done on bridge failures in the U.S. from 1980-2012 (Lee, Mohan, Haung, $\&$ Fard, 2013), bridge collapses due to earthquakes were categorized based on structural elements. The components considered were connection, girder, column, truss, foundation and bearing. The finding of the study was that 67 percent of failures happened due to the deficiency of columns and retrofitting. This shows that columns were the most vital components in the seismic resistance of bridges. 


\subsection{Selection of Representative Bridge type for Oregon}

In order to study the vulnerability of highway bridges Oregon, it is essential to have an understanding of the bridge inventory in the state. An in-depth study of the bridge inventory for Oregon is presented in this chapter. This inventory study utilizes the National Bridge Inventory (NBI) database and specific bridge plans which were attained from Oregon departments of transportation (ODOT). The subsequent sections are devoted to exploring the characteristics of the bridge database.

\subsection{Oregon Bridge Inventory}

When analyzing the of vulnerability bridges, ideally we would need to have the detailed drawings for each bridge and then generate fragility curves for all the bridges. But this is not feasible when assessing a state wide study because it is highly time consuming and not economical. However, since many bridges that are in the same region are similar, bridges can be grouped into a general bridge class.

The NBI (National Bridge Inventory) database provides information to allow for general classification of bridges. This information is contained in three of the 116 fields in the NBI. The bridges are assigned classes based on their construction material (Table 4-1), construction type (Table 4-2) and the number of spans. The Recording and Coding Guide for the Structure Inventory and Appraisal of the Nation's Bridges serves as a guide to the NBI and lists possible construction materials and types.

In the 2010 ODOT inventory, there are over 9,864 bridges and culverts. The state highway agency owns 5,280 of these. However, only 2,567 of the highway bridges are in 
the NBI database of which 1,997 are multi span. This study focused on multi-span bridges that were built before 1990. Table 4-3 shows the bridge classes and proportions of these multi span bridges that were built before 1990. As can be seen from Table 4-3, concrete continuous stringer/girder bridges are the most common type of bridge in

\section{Oregon.}

Table 4-1 Construction Materials Listed in NBI (FHWA, 1995a).

\begin{tabular}{c|l}
\hline Code & Description \\
\hline 1 & Concrete \\
\hline 2 & Concrete Continuous \\
\hline 3 & Steel \\
\hline 4 & Steel Continuous \\
\hline 5 & Prestressed Concrete \\
\hline 6 & Prestressed Concrete Continuous \\
\hline 7 & Wood or Timber \\
\hline 8 & Masonry \\
\hline 9 & Aluminum, Wrought Iron, or Cast Iron \\
\hline 0
\end{tabular}

Table 4-2 Construction Types Listed in NBI (FHWA, 1995a).

\begin{tabular}{c|l|c|l}
\hline Code & Description & Code & Description \\
\hline \hline 01 & Slab & 13 & Suspension \\
\hline 02 & Stringer/Multi-beam or Girder & 14 & Stayed Girder \\
\hline 03 & Girder and Floor beam System & 15 & Movable - Lift \\
\hline 04 & Tee Beam & 16 & Movable - Bascule \\
\hline 05 & Box Beam or Girders - Multiple & 17 & Movable - Swing \\
\hdashline 06 & Box Beam or Girders - Single or Spread & 18 & Tunnel \\
\hdashline 07 & Frame & 19 & Culvert \\
\hdashline 08 & Orthotropic & 20 & Mixed Types \\
\hdashline 09 & Truss - Deck & 21 & Segmental Box Girder \\
\hdashline 10 & Truss - Thru & 22 & Channel Beam \\
\hline 11 & Arch - Deck & 00 & Other \\
\hline 12 & Arch - Thru & & \\
\hline
\end{tabular}


Table 4-3 Number of Bridges per Class

\begin{tabular}{lcc}
\hline Bridge Types & Number & Percentage \\
\hline \hline Concrete Continuous Stringer/Girder & 523 & $28.93 \%$ \\
Prestressed Concrete Stringer/Girder & 231 & $12.78 \%$ \\
Concrete Continuous Multiple Box Beam & 145 & $8.02 \%$ \\
Concrete Continuous Slab & 139 & $7.69 \%$ \\
Prestressed Concrete Slab & 119 & $6.58 \%$ \\
Steel Stringer/Girder & 105 & $5.81 \%$ \\
P/S Concrete Continuous Multiple Box Beam & 73 & $4.04 \%$ \\
P/S Concrete Continuous Stringer/Girder & 61 & $3.37 \%$ \\
Steel Continuous Stringer/Girder & 59 & $3.26 \%$ \\
Wood or Timber Stringer/Girder & 51 & $2.82 \%$ \\
Concrete Stringer/Girder & 50 & $2.77 \%$ \\
Concrete Channel Beam & 39 & $2.16 \%$ \\
Steel Truss-Thru & 32 & $1.77 \%$ \\
Steel Truss-Deck & 23 & $1.27 \%$ \\
Steel Continuous Multiple Box Beam & 20 & $1.11 \%$ \\
Concrete Arch-Deck & 19 & $1.05 \%$ \\
Prestressed Concrete Multiple Box Beam & 15 & $0.83 \%$ \\
Steel Continuous Girder-Floorbeam & 15 & $0.83 \%$ \\
Steel Continuous Frame & 12 & $0.66 \%$ \\
Concrete Continuous Girder-Floorbeam & 10 & $0.55 \%$ \\
Other & 67 & $3.71 \%$ \\
\hline Total & 1808 & $100 \%$ \\
\hline
\end{tabular}

\subsection{Bridge Class Statistics}

The NBI provides general information on the bridge classes but actual bridge drawings are needed to assign typical details to each class. From the data provided in the database, the information such as number of spans, number of lanes, maximum span length, deck width, skew angle, year built/rebuilt, etc. can be inferred. Since multi-span concrete continuous girder (MSCG) bridges are the most common type of brides in Oregon, the study of bridge characteristic focused on these 523 bridges. 


\subsubsection{Number of Spans}

To examine the frequency of data at each span length a probability mass function (PMF) was generated. PMF is the probability that a discrete random variable $\mathrm{X}$ takes on a particular value $\mathrm{x}, \mathrm{P}(\mathrm{X}=\mathrm{x})$. Here bridges of equal number of spans were grouped together and counted and each group was then divided by the total number of bridges.

Figure 2-1 shows the PMF of MSCG multi-span bridges built before 1990.

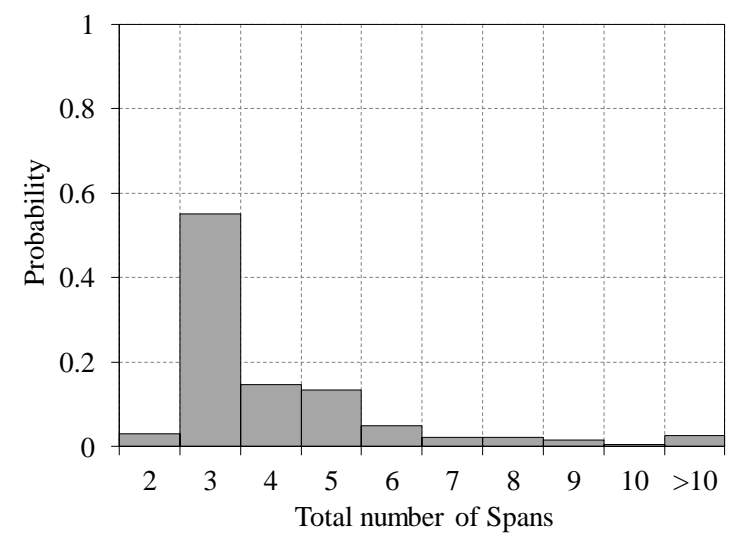

Figure 4-1 PMF of multi span MSCG bridges built before 1990

Table 4-4 Statistics of Number of Spans for major concrete bridge classes

\begin{tabular}{lcccc}
\hline Bridge Class & Mean & Std. Dev. & Median & Mode \\
\hline \hline Concrete Continuous Stringer/Girder & 4.28 & 3.2 & 3 & 3 \\
Prestressed Concrete Stringer/Girder & 3.92 & 2.01 & 3 & 3 \\
Concrete Continuous Multiple Box & 4.35 & 2.64 & 3 & 3 \\
Beam & 3.8 & 2.92 & 3 & 3 \\
Concrete Continuous Slab & 3.6 & 2.26 & 3 & 3 \\
Prestressed Concrete Slab & & & & \\
\hline
\end{tabular}


Figure $4-1$ shows that over $50 \%$ of the bridges have three spans. And as it can also be seen from Table 4-4, the most probable number of span number for all the other major concrete bridge classes is three as well. Therefore, it was safe to assume a representative MSCG bridge in Oregon should have three spans.

\subsubsection{Number of Lanes}

The PMF of number of lanes for MSCG bridges can also be generated to study the characteristics of the database with regard to number of lanes. From Figure 4-2, we can easily see over $80 \%$ of the bridges have two lanes.

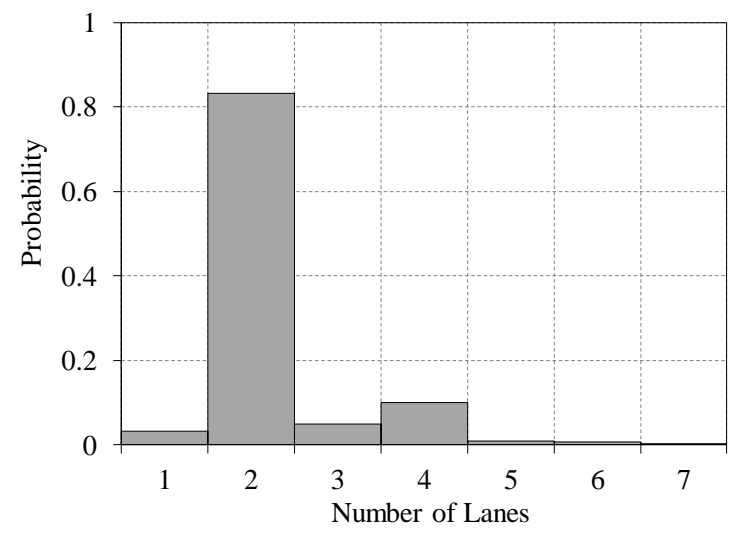

Figure 4-2 PMF of number of lanes

\subsubsection{Length of Bridge}

The empirical cumulative distribution functions (CDFs) for the length of the MSCG bridge class give a complete description of the data distribution and is shown on Figure 4-3. The average total length for the MSCG is $123 \mathrm{ft}(38 \mathrm{~m})$, with a standard deviation of $39 \mathrm{ft}(12 \mathrm{~m})$ and median value of $123 \mathrm{ft}(38 \mathrm{~m})$. This shows that half the bridges have lengths longer than $123 \mathrm{ft}$ and the other half have lengths shorter than $123 \mathrm{ft}$. 
Figure 4-4 also shows that of these MSCG bridges have a length in the range 120 to 140 feet.

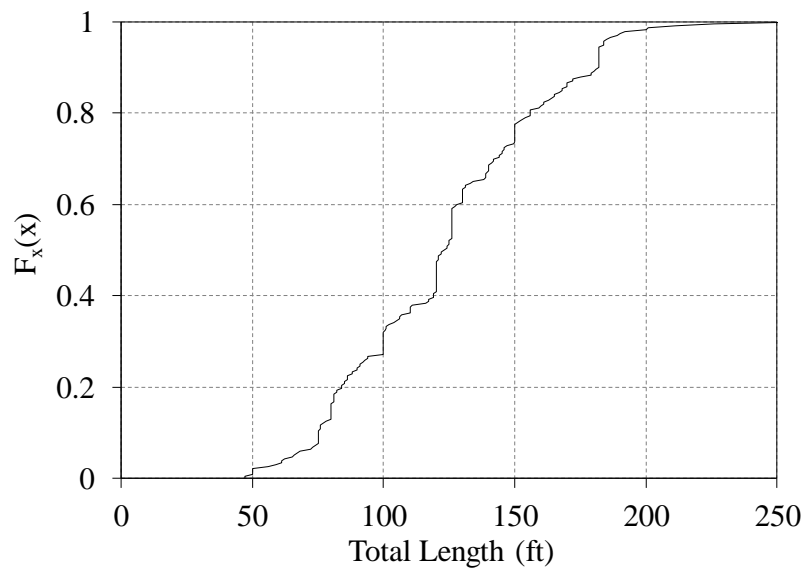

Figure 4-3 Cumulative Distribution Function for the Lengths

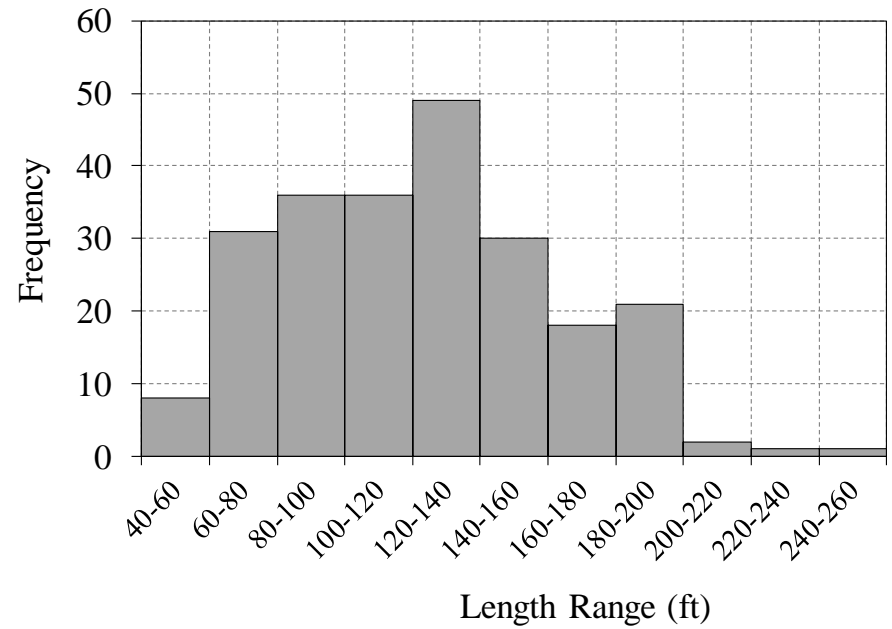

Figure 4-4 Frequency Plot of Bridge Length 


\subsubsection{Width of Bridge}

The deck width is measured in feet and is used for modeling purposes later in this study. The deck widths of the majority of the concrete continuous stringer/girder bridge classes range on average between 30 and 35 feet as can be seen from Figure 4-5.

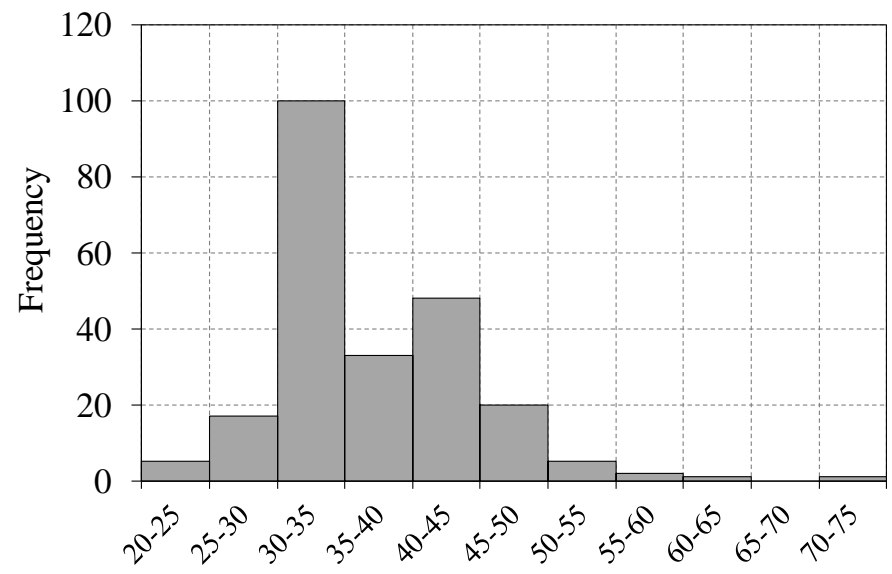

Width Range

Figure 4-5 Frequency Plot of Bridge Width

\subsubsection{Column Height}

Column height greatly affects the sensitivity of the seismic response of a bridge. Since the NBI does not record column heights, column heights were extracted from available bridge drawings. From what can be seen in Figure 4-6, the majority of columns have a height in the range 15 to 25 feet. 


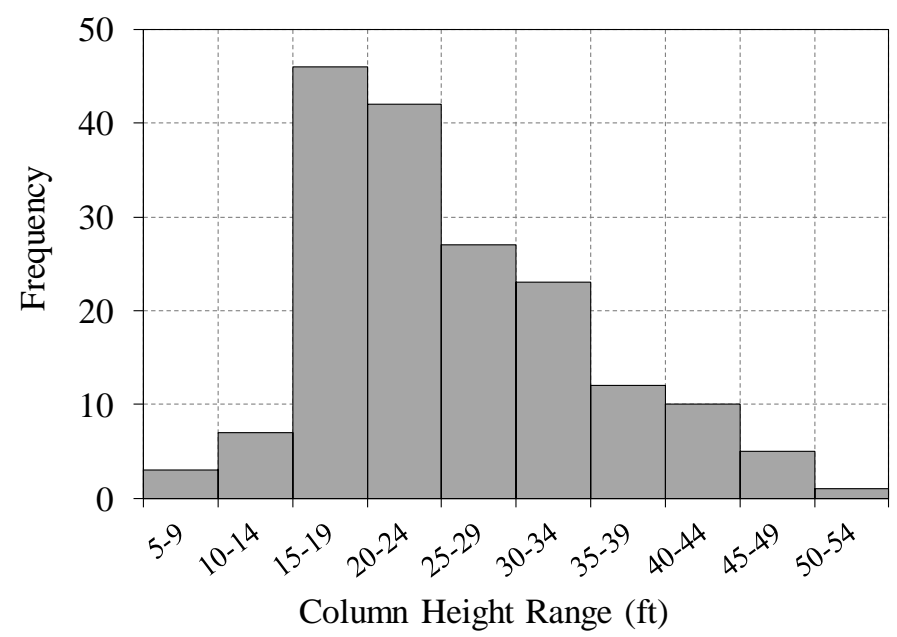

Figure 4-6 Frequency Plot of Bridge Column Height

\subsubsection{Number of Columns per bent}

The number of columns per bent is also another substructure detail to be considered. Of the bridge drawings that were available, almost $75 \%$ of the multi-span continuous concrete girder bridges were found to have two or four columns per bent.

\subsubsection{Reinforcement Details}

For majority of the bridges, the columns use 4 - \#8, \#9 or \#10 bars to provide longitudinal reinforcement. The transverse reinforcement is provided by \#3 bars spaced at 12 inches center to center for over $80 \%$ of these multi-span continuous concrete girder bridges.

\subsection{Representative Bridge Type for Oregon Inventory}

After taking a closer look at ODOT's bridge inventory, a representative bridge for Oregon would be a multi-span continuous concrete girder bridge (MSCG) with three 
spans. The bridge would have two lanes, and the length and width of the bridge would be between 120 and 140 feet and 30 and 35 feet respectively. The columns would have four No. 8, No. 9 or No. 10 longitudinal reinforcements and No. 3 transverse reinforcing hoops typically at 12 inches on center anchored by $90^{\circ}$ hooks.

\subsection{Retrofit Measures}

Reinforced concrete columns constructed before 1971 are commonly deficient in flexural ductility and shear strength as bridges were designed primarily for gravity loads without much consideration to lateral forces from seismic loading. These columns lack sufficient transverse reinforcement to provide satisfactory performance in a major seismic event. As was mentioned in the previous section, typically, No. 3 or No. 4 hoops at 12 inches on center were provided in columns regardless of the column cross-sectional dimensions. The stirrups were anchored by $90^{\circ}$ hooks with short extensions and intermediate ties were seldom used. All these details contribute to the columns' deficiency and make the columns vulnerable to shear failure and provide limited flexure capacity since the hoops do not provide sufficient confinement. The longitudinal reinforcements could also buckle since the hoops provide minimal restraint once the concrete coves spalls.

Bridges built prior to 1971 also have inadequate lap splices, which normally occur in the potential plastic hinge zone at the base of the column. This detail can also potentially be a cause for reduced column ductility and can result in rapid loss of flexural strength of the column. 
The ability of structures to achieve adequate deformation capacity plays a significant role in the prevention of structural failures in seismic events. The deformation capacity of existing bridges can be enhanced by modifying certain substructure elements and connections. Bridge columns are typically retrofitted to increase the overall ductility of the bridge. The performance of seismically vulnerable bridge columns can be upgraded using various techniques including reinforced concrete jacketing, steel jacketing, active confinement by prestressing wire, and composite fiber/epoxy jacketing.

\subsubsection{Retrofit Options for Deficient Columns}

\subsubsection{Steel Jacketing}

Steel jacketing was originally developed for circular columns. Previous research studies (Chai, Priestley, \& Seible, 1991) have shown that steel jacketing is an effective retrofit technique for seismically-deficient concrete columns. Details of a typical steel jacket retrofit on a circular column is shown in Figure 4-7. Based on satisfactory laboratory results, steel jackets have been employed to retrofit both circular and rectangular columns around the world. For rectangular columns, the recommended procedure is to use an oval jacket (Figure 4-9), which provides continuous confining action similar to that for a circular column. Rectangular steel jackets are also effective in enhancing shear resistance of columns. These jackets can improve column ductility by eliminating the brittle shear mode of failure. The failure mode may shift to a flexural mode for which the rectangular jacket can provide only limited assistance, since the confining action of the rectangular jackets can only be developed as a result of lateral bending of the jacket sides, which is a very flexible action compared to the membrane 
action developed in an oval or circular jacket. Detailed design guidelines for steel jacketing are found in the Seismic Retrofitting Manual for Highway Structures (FHWA, 2006).

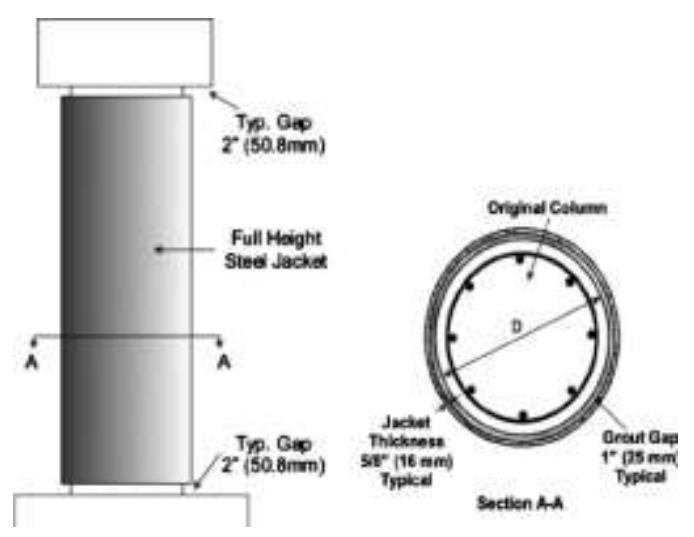

Figure 4-7 Details of a typical circular column steel jacket (Wright, Desroches, \& Padgett, 2010)

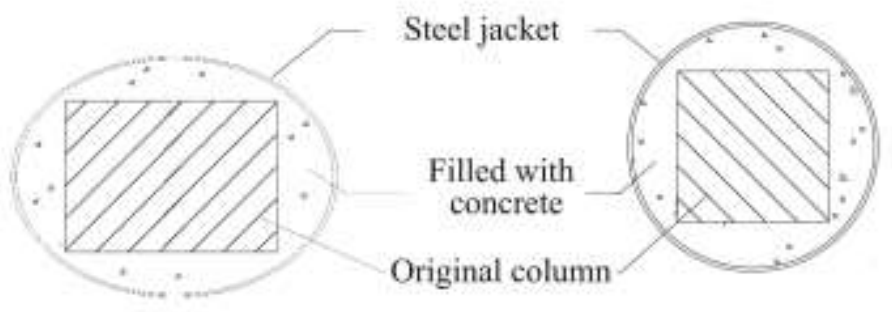

Figure 4-8 Details of a typical rectangular and circular column retrofitted with a steel jacket

\subsubsection{Concrete Jacketing}

This application follows the same principles as concrete design. The concrete jacket is applied by drilling holes in the existing column and lateral ties are placed through these holes and are anchored on both sides of the column. Concrete jackets have been used to force yielding away from the location of starter bar splices, and in such cases, the need to improve the performance of the lap-splice has been avoided. 
Experimental investigations by (Rodriguez \& Park, 1994) showed significant improvement of strength and stiffness over as built columns compared to retrofitted damaged and retrofitted undamaged columns. Concrete jackets increase the flexural strength and stiffness of a column even more than a steel jacket, which can cause undesirable effects on bridge performance (FHWA, 2006). Retrofit using a fiberreinforced concrete sleeve can potentially perform better than steel jackets in areas of corrosive environment and are a better choice in certain areas (Dunwoodie, 1997).

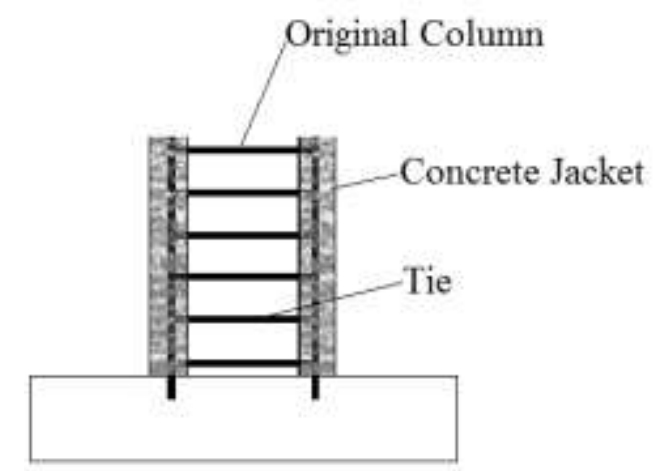

Figure 4-9 Typical concrete jacket retrofit details

\subsubsection{Angle and Rod Hoop Retrofit}

In the angle and rod hoop retrofit measure, steel angles are placed at each corner of the column and connected by threaded rods that act as hoops spaced along the specimen. These hoops provide confinement under cyclic loading and provide shear reinforcement. In a laboratory experiment, (McLean \& Bernards, 1992) found that strength and ductility of columns were moderately improved. Tested specimens showed an increase in lateral load capacity of about seven percent. Smaller hoop spacing resulted in larger ductility capacity and a slower internal tie yielding. 


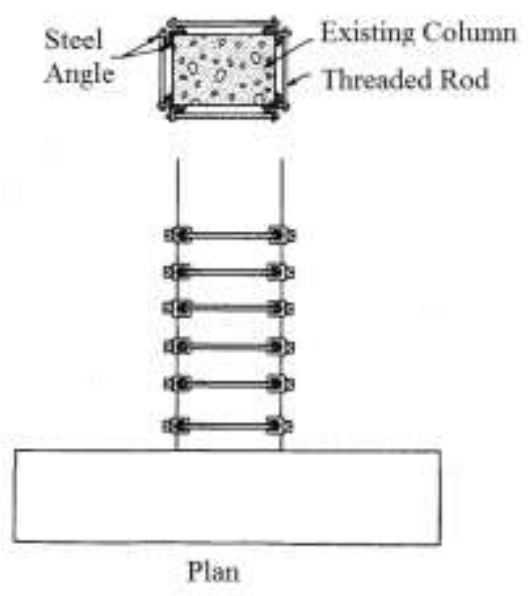

Figure 4-10 Angle and rod hoop retrofit

\subsubsection{External Prestressing Steel}

External prestressing steel wires under tension are wrapped around a column to achieve confinement. This retrofit method has successfully increased the flexural ductility of circular columns with lap splices at the critical section, but its effect on shear strength has not yet been quantified (FHWA, 2006).

An advantage of this practice is that it does not affect the flexural strength and stiffness of the columns. However, the high cost of designing a machine that is big enough to produce the required tension to wrap prestressing wire around the columns makes it uneconomical. Saatcioglu \& Yalcin (2003) applied external prestressing to columns in transverse direction using individual hoops that consist of prestressing strands and specially designed anchors. They tested the columns under constant axial compression and incrementally increasing lateral deformation reversals. Their results show that this retrofit methodology can reduce shear failure and increase flexural capacity and inelastic column deformability. 

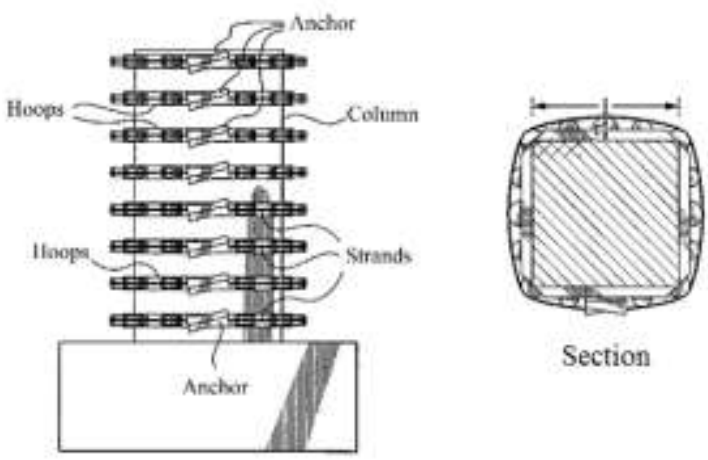

Figure 4-11 Elevation and cross-section of external prestressing steel retrofit.

\subsubsection{Composite Material Retrofitting}

Recent developments in the manufacturing of fiber reinforced polymer (FRP) composite materials have made these materials available for a wide range of applications, including seismic retrofit of reinforced concrete columns. Compared to steel and concrete jacketing, FRP wrapping has several advantages, including very low weight-to-strength ratios, high elastic moduli, resistance to corrosion, and ease of application. FRP can improve column ductility without considerable stiffness amplification while maintaining the bridge dynamic properties (Haroun \& Elsanadedy, 2005).

Carbon fiber reinforced polymer (CFRP), glass fiber reinforced polymer (GFRP) and aramid fiber reinforced polymer (AFRP) are the most commonly used FRP composite materials. CFRP has a higher modulus of elasticity and has the highest tensile strength. The Washington State Department of Transportation recommends using CFRP to retrofit bridges because it is less affected by moisture than GFRP (WSDOT, 2006).

FRP retrofit systems can be effective for both circular and rectangular columns. However, circular jackets provide a circular column with a continuous confinement 
pressure, whereas rectangular jackets provide confinement pressure at the corners only. Hence, the corners of rectangular columns are typically rounded prior to retrofitting to avoid stress concentrations at the corners (Seible, Hegemier, Priestley, Innamorato, \& Ho, 1995).

Endeshaw, et. al. (2008) saw that columns retrofitted with CFRP jackets designed based on ACTT-95/08 recommendations for rectangular-shaped retrofits resulted in satisfactory performance, but bulging of the CFRP jacket was observed towards the end of testing. They increased the thickness of CFRP jackets, which resulted in reduced bulging of the CFRP jacket. They also retrofitted a specimen with a CFRP jacket designed based on $150 \%$ of the ACTT-95/08 recommendations, and they observed that it improved the column's performance.

\subsubsection{Recommended Retrofit Option}

After careful consideration other retrofit practices, glass and carbon composite material options were further investigated. The design was performed to estimate the number of layers for lap-splice confinement and the anticipated displacement ductility of both FRP System scenarios including the use of 1, 2, and 3 layer systems of both glass and carbon FRP system. The materials considered are Tyfo ${ }^{\circledR}$ fiberwrap systems from Fyfe Co. LLC. Summary of the results is given in Table 4-5

Table 4-5 Column Ductility Summary

\begin{tabular}{l|c|c|c}
\hline \multirow{2}{*}{ Material } & \multicolumn{3}{|c}{ Displacement Ductility per Layer } \\
\cline { 2 - 4 } & 1 & 2 & 3 \\
\hline \hline Tyfo $®$ SEH-51A (glass fiber) & 6.9 & 10.2 & 13.2 \\
\hline Tyfo $®$ SEH-41 (carbon fiber) & 5.5 & 7.4 & 9.2 \\
\hline
\end{tabular}


The carbon fiber system provides adequate ductility and was selected for retrofit. The expectation from this retrofit method was to adequately increase the ductility of the plastic hinge zone so that the region does not prematurely degrade leading to a possible shear failure and lap splice failure.

\subsection{Experimental Investigation}

The experimental program consisted of three test specimens intended to represent full-scale models of typical bridge columns supporting multi-column bents as constructed prior to 1970s. All three specimens had the same material properties, cross-sectional dimensions and reinforcement ratios. The variables in the testing program were the loading protocols and column retrofit conditions. The cyclic performance of these specimens was intended to reveal vulnerabilities in the existing columns and compare their performance under different loading protocols and to establish benchmarks to evaluate the effectiveness of the applied retrofit measures.

\subsubsection{Specimens Details}

Each specimen comprised a 610 x 610 x $2540 \mathrm{~mm}(24 \times 24 \times 113$ in) cantilevered column connected to a $1654 \times 1651 \times 610 \mathrm{~mm}(65 \times 65 \times 24 \mathrm{in})$ footing. The height of the specimen corresponded to a typical column height found in the bridge inventory for Oregon up to the point of assumed inflection under double curvature. The column specimen reinforcement detailing and cross-section are illustrated in Figure 4-12 (a). The longitudinal reinforcement in each prototype column consisted of $4 \phi 32 \mathrm{~mm}$ (No. 10) bars on four corners with $\phi 10 \mathrm{~mm}$ (No. 3) stirrups with $90^{\circ}$ hooks at $305 \mathrm{~mm}$ (12 in) 
center to center spacing and $50 \mathrm{~mm}$ ( 2 in) of clear cover concrete confining the column core. The applied vertical load was approximately 7 percent of the gross cross-sectional axial strength for the columns. The columns in the experimental program were given an identification that denoted by column condition ( $\mathrm{A}=\mathrm{As}$-built, $\mathrm{R}=$ Retrofitted) and applied loading protocol $(\mathrm{C}=$ Conventional, $\mathrm{S}=$ Subduction), resulting in the identifications for the three columns as A-C, A-S and R-S.

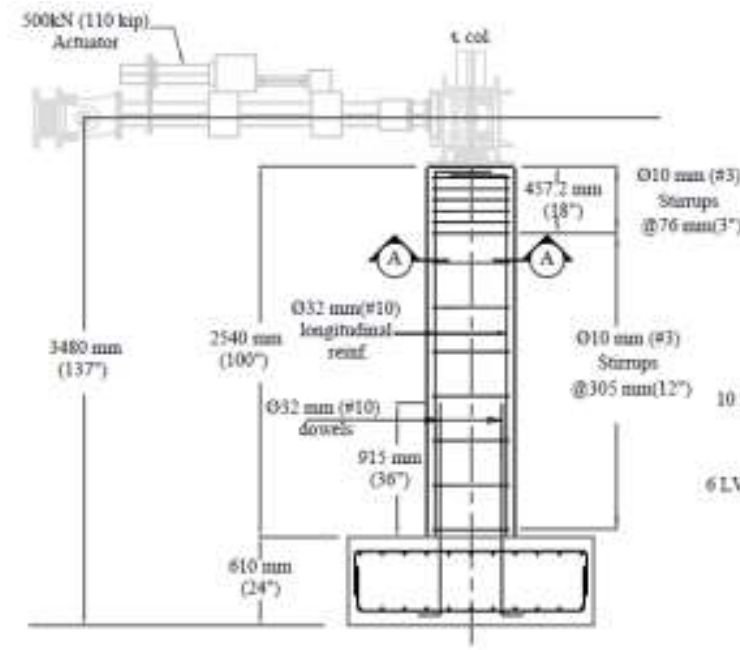

(a)

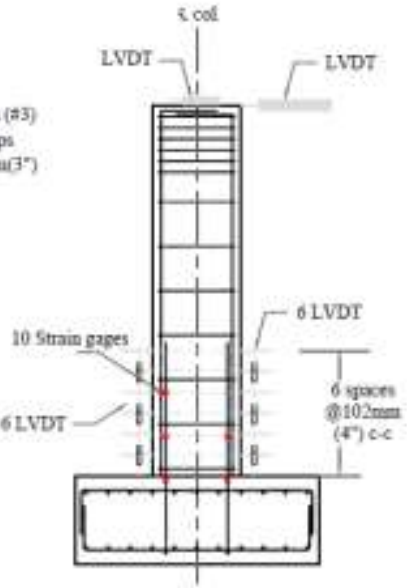

(b)

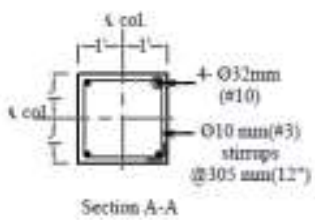

Figure 4-12 (a) Test setup and reinforcement details; (b) instrumentation details

\subsubsection{Material Properties}

All three columns were cast at the same time with one batch of concrete for the footings and another batch for the columns. Normal weight concrete was used to construct the test specimens with a target 28-day strength of $22.75 \mathrm{MPa}$ (3300 psi), based on a common mix bridge specification prior to 1970s. Standard compression testing of $152 \mathrm{~mm}$ ( 6 in) by $305 \mathrm{~mm}$ (12 in) concrete cylinders were performed at approximately 7day intervals up to 28 days and again at the day of test completion. 
All reinforcing steel used to construct the test specimens consisted of Grade 60 deformed bar conforming to the American Society of Testing and Materials (ASTM) Designation A615. Longitudinal reinforcement consisted of four $\phi 32 \mathrm{~mm}$ (No. 10) bars and column ties were created from $\phi 10 \mathrm{~mm}$ (No. 3) bars, and footing reinforcements were composed of $\phi 19 \mathrm{~mm}$ (No. 6) bars. The column cage and footing cage were assembled in place. The columns were constructed with lap splices where dowels from the footing overlapped the longitudinal rebar in the column by $914 \mathrm{~mm}$ (36 in). The provided lap splice length is below the modern detailing recommendations (ACI 318 (2014), where the tensile stress exceeding $0.5 f_{y}$, requires a Class B splice lap of $1.31_{d}$, where $l_{d}$ is the tension development length. This would result in a lap splice requirement of $68 \mathrm{~d}_{\mathrm{b}}$ equal to $2210 \mathrm{~mm}$ (87 in). The $\phi 10 \mathrm{~mm}$ (No. 3) lateral ties were spaced at 305 $\mathrm{mm}$ (12 in) and included $90^{\circ}$ hooks to represent typical pre-1970s column design details. The reinforcement characteristics were obtained from tension tests and are reported on Table 4-6.

Table 4-6 Reinforcing Steel Properties

\begin{tabular}{lccc}
\hline & & $\begin{array}{c}\text { Yield Strength } \\
(0.2 \% \text { offset })\end{array}$ & Tensile Strength \\
\cline { 3 - 4 } Bar Size & $\begin{array}{c}\text { Grade } \\
\text { MPa (ksi) }\end{array}$ & MPa (ksi) & MPa (ksi) \\
\hline \hline$\phi 9.5 \mathrm{~mm} \mathrm{( \# 3)}$ & Gr. 420 (60) & $414(60)$ & $643(93.2)$ \\
$\phi 19 \mathrm{~mm} \mathrm{( \# 6)}$ & Gr. 420 (60) & $522(75.7)$ & $760(110.2)$ \\
$\phi 32 \mathrm{~mm} \mathrm{( \# 10)}$ & Gr. 420 (60) & $485(70.3)$ & $696(100.9)$ \\
\hline
\end{tabular}

\subsubsection{Retrofitting Material}

For this project a CFRP composite material called Tyfo®SCH-41 was used for retrofit and was selected due to its many advantages. TyfoßSCH-41 composite is 
comprised of epoxy and reinforcing carbon fabric and has unidirectional carbon fabric orientated in the $0^{0}$ direction. One major advantage is the capability to improve column ductility without considerable stiffness amplification while maintaining the bridge dynamic properties. The expectation from this retrofit method is to adequately increase the ductility of the plastic hinge zone so that the region does not degrade leading to a possible shear failure and lap splice failure. Preliminary design calculations show that three layers of the Tyfo SCH-41 composite would give a displacement ductility factor of 9.3. Therefore, three layers of this CFRP were wrapped from top of the footing up to the potential plastic hinge region ( $\sim 3$ feet $)$ and were provided in order to enhance the deformation capacity of the columns as can be seen in Figure 4-13.

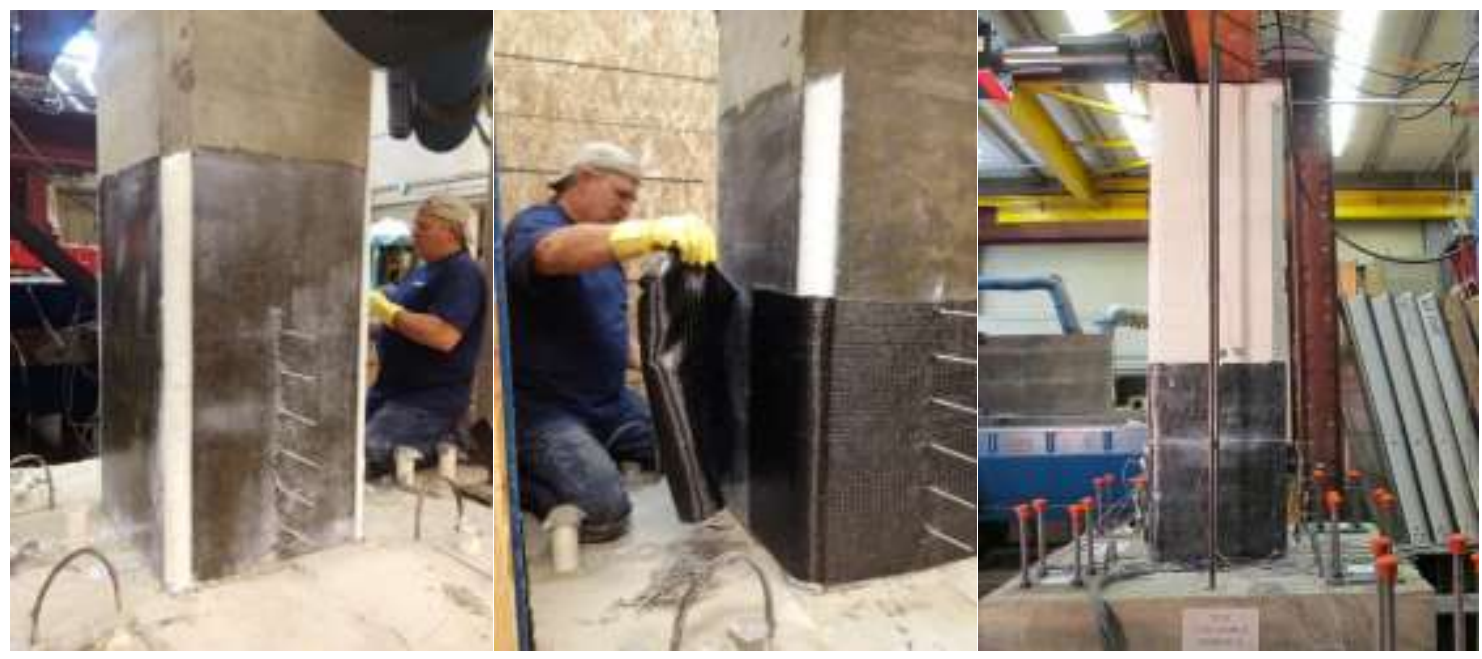

Figure 4-13 CFRP Retrofit Application 


\subsubsection{Test Setup and Load History}

All specimens were instrumented to monitor strains, deformations and applied forces as outlined in Figure 4-12 (b). Strain gauges were used to measure the strain at specific points in the specimen. The strain gauges were mounted on the flexural reinforcing bars prior to the placement of the concrete. All strain gauges were placed at and below the plastic hinge, which was expected to be approximately $610 \mathrm{~mm}$ (24 in) above the footing. A total of 12 strain gauges were mounted on the flexural reinforcing bars, 2 on the longitudinal bars and 10 on the dowels extending from the foundation. Linear variable differential transducers (LVDTs) were used to measure column rotation up to $610 \mathrm{~mm}$ ( $24 \mathrm{in}$ ) height from the base. LVDTs located at the base of the columns at heights $102 \mathrm{~mm}, 203 \mathrm{~mm}, 305 \mathrm{~mm}, 406 \mathrm{~mm}, 508 \mathrm{~mm}$ and $610 \mathrm{~mm}$ (4 in, 8 in, 12 in, 16 in, $20 \mathrm{in}$, and $24 \mathrm{in}$ ) measured from the top of footing in an effort to measure relative curvature at each of the heights up the column. Additional LVDTs were used to measure column-base slippage and uplift. In addition to monitoring the displacement of the actuator, another LVDT was placed on top of the column to directly measure the column displacement relative to the base and independently of any potential flexibility of the lateral reaction wall.

The columns were tested in the upright position as illustrated in Figure 4-12 (a). The top of the column was free to translate and rotate to reflect the appropriate boundary condition of the assumed inflection point. The cyclic lateral deformations were applied using a horizontal actuator that was displacement controlled and included an integrated load cell to monitor the load throughout the experiment. To simulate the imposed dead 
load on the columns, axial load was applied at the beginning of each test and kept relatively constant during the test by means of hydraulic rams. The target nominal axial load ratio $P /\left(f^{\prime}{ }_{c} A_{g}\right)$ was 0.07 for all three columns. This nominal value was calculated based on a nominal $\mathrm{f}_{\mathrm{c}}{ }_{\mathrm{c}}$ value of $24 \mathrm{MPa}(3500 \mathrm{psi})$, resulting in axial load of $667 \mathrm{kN}$ (150 kip). The experimental program test matrix and measured material strengths are reported on Table 4-7. The axial load was applied through four high-strength rods installed beside the column, attached to a horizontal steel transfer beam and anchored into the column footing. The footing was secured to the laboratory floor with post-tensioning rods. The hydraulic rams were connected in line with a hydraulic accumulator in order to minimize the increase in axial load caused by the geometric elongation of the high-strength rods under large lateral deformations of the column. Load cells were used to monitor the applied axial load during the experiments and were found to increase by approximately $20 \%, 21 \%$ and $16 \%$ at the point of failure for columns A-C, A-S and R-S respectively. This increase was substantially less for lower deformations.

Table 4-7 Experimental Program Test Matrix and Measured Material Strengths

\begin{tabular}{c|c|c|c|c|c}
\hline \multirow{2}{*}{ Column } & \multirow{2}{*}{$\begin{array}{c}\text { Axial Load } \\
\text { Ratio (nominal) } \\
(\%)\end{array}$} & $\begin{array}{c}\text { Axial Load } \\
\text { Ratio } \\
\end{array}$ & $(\%)$ & \multirow{2}{*}{$\begin{array}{c}\text { Loading } \\
\text { Protocol }\end{array}$} & \multicolumn{2}{|c}{ Measured Material Strength } \\
\cline { 5 - 6 } & 7.4 & 5.9 & Conventional & $30.4(4.42)$ & $485(70.3)$ \\
\hline \hline A-C & 7.4 & 6.2 & Subduction & $28.8(4.17)$ & $485(70.3)$ \\
\hline A-S & 7.4 & 5.5 & Subduction & $32.0(4.67)$ & $485(70.3)$ \\
\hline R-S & & & & & \\
\hline
\end{tabular}

Commonly, the seismic performance of reinforced concrete bridge columns is assessed using a cyclic loading protocol such as the one shown in Figure 4-14(a). Given 
the pervasive use of this type of protocol in assessing cyclic capacity of columns, this protocol is referred to as conventional. Quasi-static loading protocols that aim to assess the behavior of bridge columns subjected to subduction zone earthquakes have been developed through analysis of strong motion records (Bazaez \& Dusicka, 2016) and were incorporated into this study. This subduction zone loading protocol is illustrated in Figure 4-14(b) and intends to capture more closely the amplitude and the number of cycles in RC columns. As such, the subduction earthquake based protocol has the potential to improve the assessment of bridge column capacity for bridges within the subduction zone hazard. For performance comparison, both the conventional and the subduction loading protocols were utilized. In order to control the amplitude of each cycle displacement ductility factors $(\mu)$ were defined as $\mu=\delta / \delta_{y}$, where $\delta$ is the top lateral displacement and $\delta_{\mathrm{y}}$ is the effective experimental yield displacement of the system. In both cases, the maximum deformations targeted displacement ductility $\mu=8$, thereby providing a common maximum displacement but a different cyclic loading history.

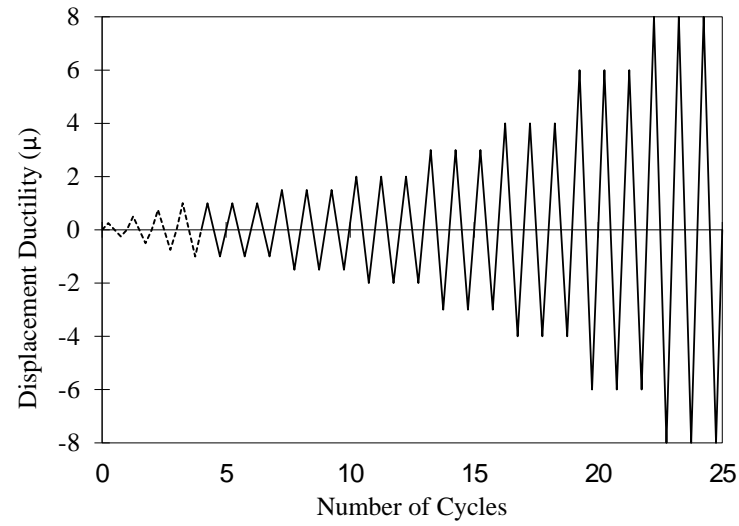

(a)

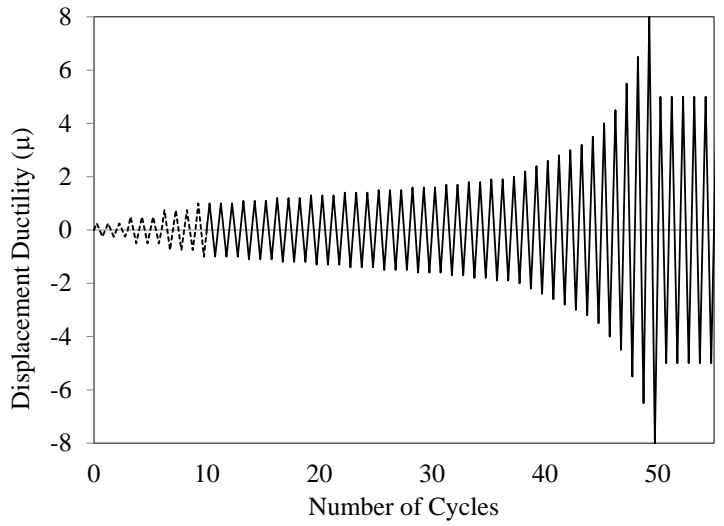

(b)

Figure 4-14 (a) Conventional Loading Protocol; (b) Subduction Loading Protocol 


\subsubsection{Yield Displacements}

Analytical column yielding was preliminarily calculated using moment-curvature analysis of the column cross-section following AASHTO (2012) recommendation.

During the tests, when the column reached this analytical first yield, the measured force and displacement were used to calculate the experimental elastic stiffness as $K_{e}=V_{y} / \delta$. Then, using the analytically obtained force at which the extreme concrete compression fiber strain reached $\varepsilon_{\mathrm{c}}=0.004$ and the experimentally obtained elastic stiffness, the effective experimental yield displacement was determined as $\delta_{y}=V_{\left(e_{c}=0.004\right)} / K_{e}$. This equivalent yield displacement method of obtaining the column yield displacement was applied to the experimental results and is consistent with numerous experimental studies. (Hines, Seible, \& Priestley, 2002), (Priestley, Calvi, \& Kowalsky, 2007), (Goodnight, Kowalsky, \& Nau., 2013).

Flexural curvature used in the preliminary analytical study using AASTHO (2012) may not adequately represent the flexibility of columns due to analysis limitations in not accounting for shear deformation and reinforcement slip (Moehle, 2015). In an effort to account for this additional shear and bond-slip deformation, analytical effective yield displacements were calculated using Equation 4-1 (Moehle, 2015) and compared to the effective yield attained experimentally.

$$
\begin{gathered}
\delta_{y}=\delta_{f}+\delta_{v}+\delta_{s} \quad \text { Equation 4-1 } \\
\delta_{f}=\frac{\phi_{y} l^{2}}{3} \text { (Flexural deformation) }
\end{gathered}
$$




$$
\begin{gathered}
\delta_{v}=\frac{V l}{A_{v} G_{e f f}} \text { (Shear deformation) } \\
\delta_{s}=\theta * l=\frac{f_{y}{ }^{2} d_{b}}{8 E_{s} u\left(d-c_{d}\right)} * l \text { (Slip Deformation) }
\end{gathered}
$$

where $\phi_{y}=$ effective yield curvature, $l=$ column height, $V=$ shear force, $A_{v}=$ effective shear area of column cross-section $=(5 / 6) \mathrm{A}_{\mathrm{g}}, \mathrm{A}_{\mathrm{g}}=$ gross area of cross-section, $\mathrm{G}_{\text {eff }}=$ effective shear modulus $=0.2 \mathrm{E}_{\mathrm{c}}, \mathrm{E}_{\mathrm{c}}=$ modulus of elasticity of concrete, $\mathrm{f}_{\mathrm{y}}=$ yield strength of reinforcing bar, $\mathrm{d}_{\mathrm{b}}=$ bar diameter, $\mathrm{E}_{\mathrm{s}}=$ modulus of elasticity of steel, $\mathrm{d}=$ distance from extreme compression fiber to centroid of longitudinal tension reinforcement, $\mathrm{c}_{\mathrm{d}}=$ distance from extreme compression fiber to neutral axis and $\mathrm{u}$ $=$ uniform bond stress $=0.5 \sqrt{f^{\prime}} M P a$. The analytical and experimental yield values for the three specimen are summarized in Table 4-8 and highlighted in Figure 4-15 for each of the tests. The effective analytical yield displacements agree well with the effective yield displacements obtained experimentally. This result exemplifies that bond-slip deformation needs to be considered when determining yield displacements analytically.

\subsection{Experimental Results}

Experimental results from the three full-scale columns were compared to determine the effects of subduction earthquake based loading history on the cyclic performance and the effectiveness of retrofit technique. Table 3 summarizes the experimentally obtained peak strengths, displacements at failure and the resulting ductility values. Failure was defined as $20 \%$ degradation of peak load at reversal. The axial loads applied at the top of the column can influence the measured lateral load at 
large deformations. Therefore, the horizontal component of the axial load, which was directly measured using load cells at all times during the experiment, has been accounted for in the computation of the total lateral load at each displacement.

Cracks in the concrete were monitored and marked following load reversals as the applied lateral load passed through zero. Most of the damage in all the columns occurred at the base of the column where the maximum moment occurred. However, numerous flexural cracks were also visible up to the mid-height of the columns and this formation of flexural cracks continued with increasing levels of displacements. The theoretical balanced axial load and balanced moment were calculated to be approximately $2200 \mathrm{kN}$ (500 kip) and $780 \mathrm{kN}-\mathrm{m}$ (580 kip-ft), respectively. However, the axial loads applied during the experiment were much lower at $667 \mathrm{kN}$ (150 kip) for all. Hence, all three columns did not reach the theoretical balanced moment. The theoretical shear capacity of the columns was also calculated to be over $450 \mathrm{kN}$ (100 kip). These values show that the columns were flexure dominated as the shear capacity was greater than the imposed shear during any of the tests.

\subsubsection{Column Displacement Capacities and Failure Modes}

For column A-C the primary mode of failure was flexural tension failure causing crushing of concrete at the base of the column, which resulted in plastic behavior to be concentrated at the cold joint between the footing and the column. The damage progression at failure is illustrated in Figure 4-16 (a). Once the cover was lost, dowels buckled at the base of the column. First yielding of the dowels in A-C was recorded at a ductility of approximately $\mu=0.8(\delta=20 \mathrm{~mm})$. The peak lateral load of $197 \mathrm{kN}(44.3$ 
kip) was recorded at a displacement ductility of approximately $\mu=2.7(\delta=66 \mathrm{~mm})$.

Column A-C reached a displacement ductility of $\mu=6.2(\delta=153 \mathrm{~mm})$ at failure.

The primary mode of failure for column A-S was by lap splice failure. The progression of damage is shown in Figure 4-16 (b). Vertical cracks consistent with lap splice failure started showing at a ductility of $\mu=1.8(\delta=46 \mathrm{~mm})$ and the cracks opened up to widths $>2.0 \mathrm{~mm}$ at a ductility of $\mu=4(\delta=100 \mathrm{~mm})$. The concrete cover above the lap splice showed only minimal signs of vertical cracking. Spalling of the concrete cover at the base of the column began due to flexural loading, exposing the column reinforcement. Once the concrete cover was lost, the dowels in this region began to buckle. There was also visible rocking at the cold joint between the column and the footing. First yielding of the dowels in A-S was recorded at a ductility of approximately $\mu=0.7(\delta=17 \mathrm{~mm})$. The peak lateral load was $178.5 \mathrm{kN}$ (40.1 kip) and occurred at a ductility of $\mu=2.3$ ( $\delta=57 \mathrm{~mm}$ ). As can be seen on Figure 4-15 (b), there was a higher degradation in the specimen in the positive side of loading application. At failure, Column A-S reached a displacement ductility of 4.4 and 6 with lateral loads $143 \mathrm{kN}(32.1$ kip) and $-138 \mathrm{kN}(-31.1 \mathrm{kip})$ at positive and negative load reversals, respectively.

Column R-S was able to attain a displacement ductility, $\mu$, greater than 8 and did not fail during the experiment due to testing limitations. The peak lateral load was 253 $\mathrm{kN}$ (57 kip) and occurred at a ductility $\mu=8.2(\delta=187 \mathrm{~mm})$. Similar to specimens A-C and A-S, there was significant rocking at the cold joint between the column base and footing (Figure 4-16), and horizontal cracks were observed on the faces of the columns as well as diagonal shear cracks. The test on R-S showed that retrofitting with CFRP wraps 
considerably enhanced displacement ductility of these deficient columns. The retrofit helped inhibit bond failures in lap splices of longitudinal reinforcement in the plastic hinge regions and provided confinement producing very minimal spalling of concrete at the base of the column. 


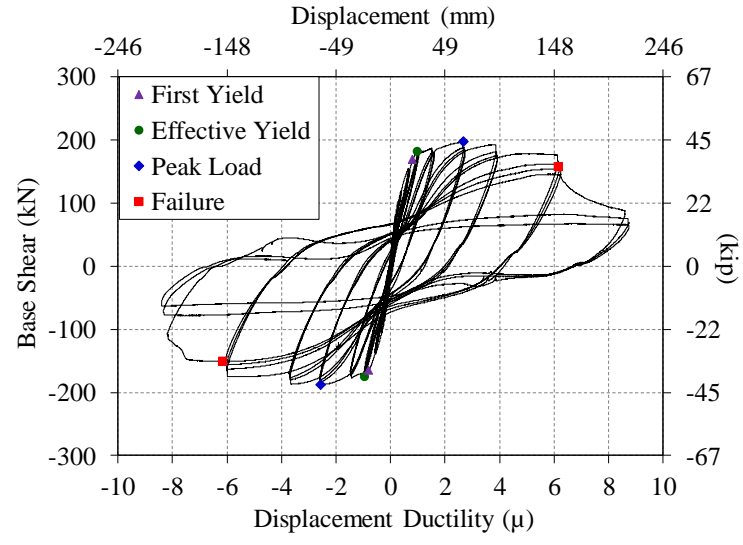

(a)

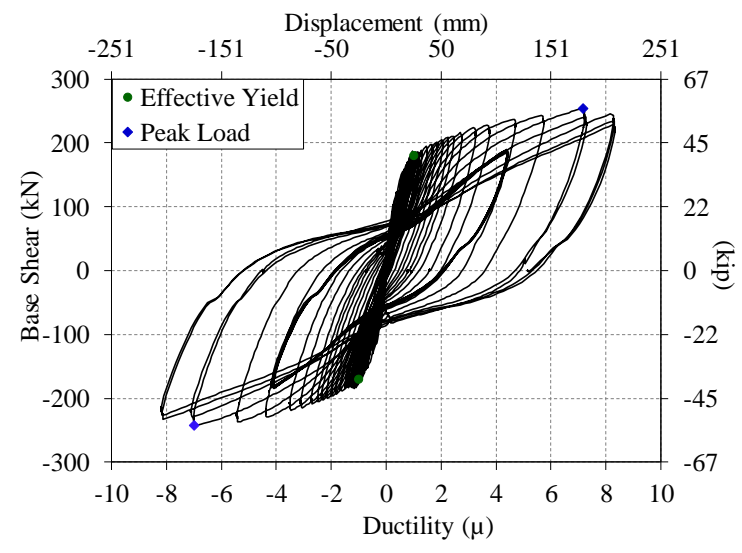

(c)

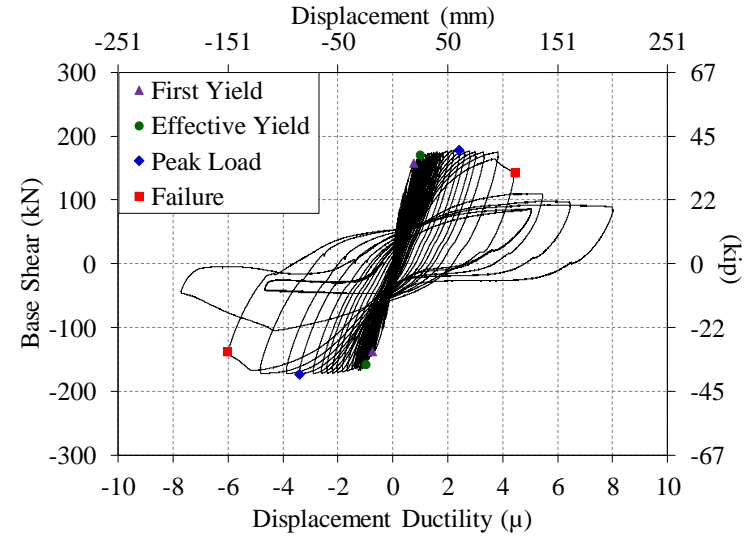

(b)

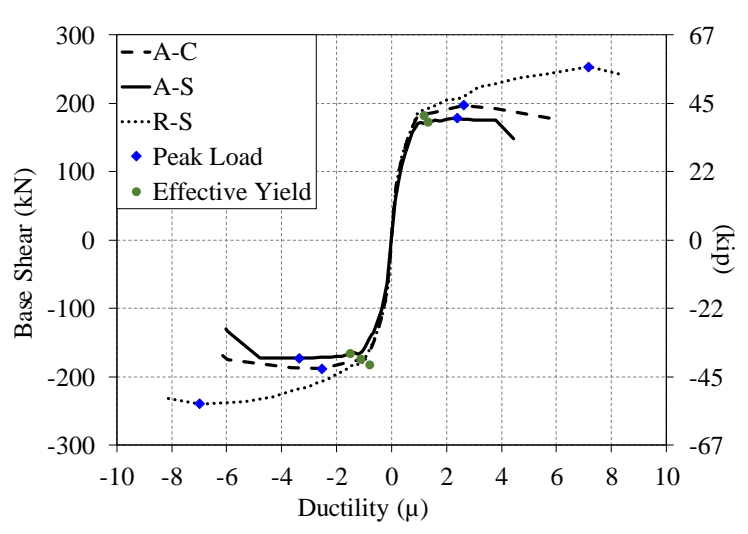

(d)

Figure 4-15 Hysteretic curves of (a) A-C; (b) A-S; (c) R-S; (d) Backbone Comparison 

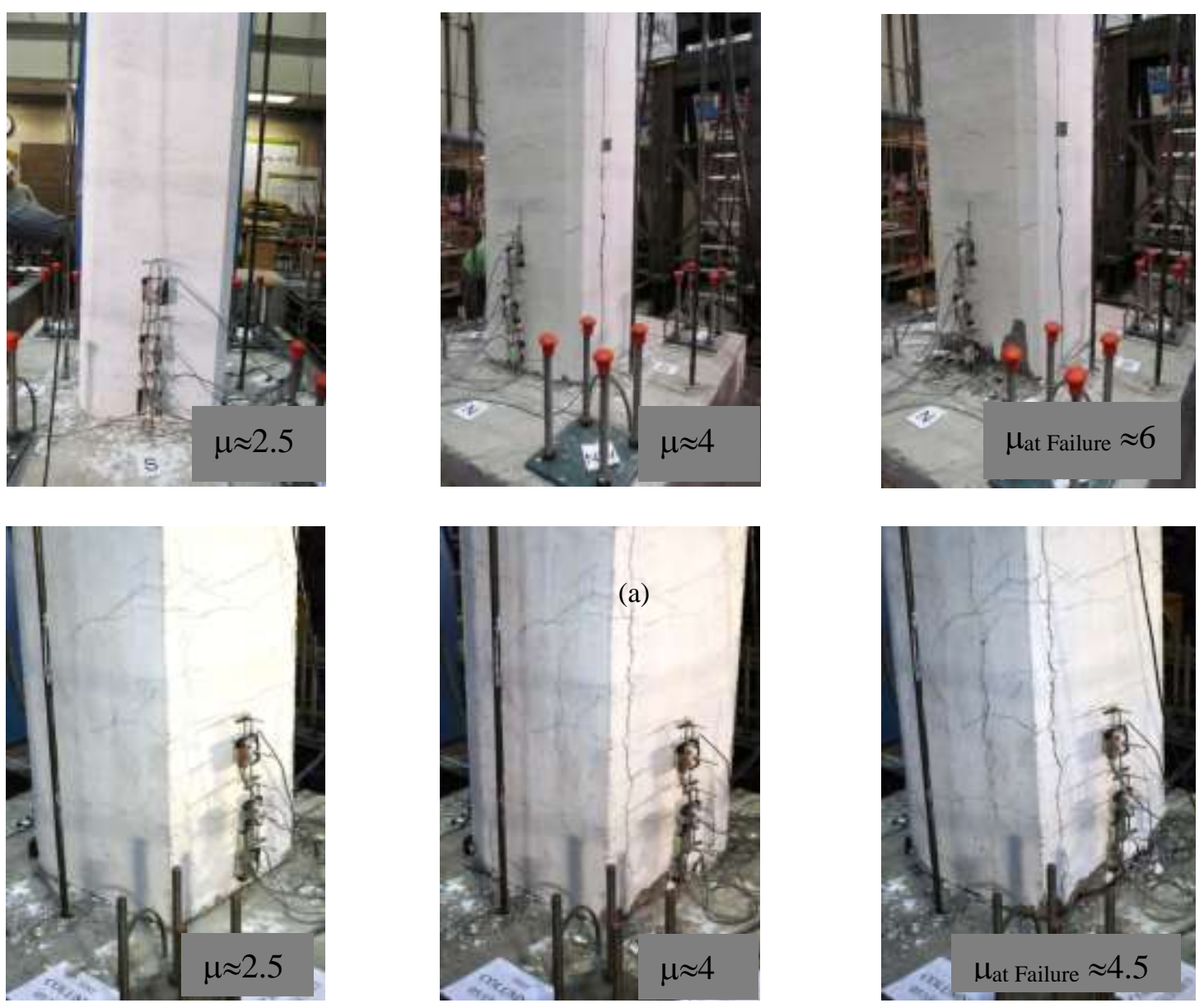

(b)
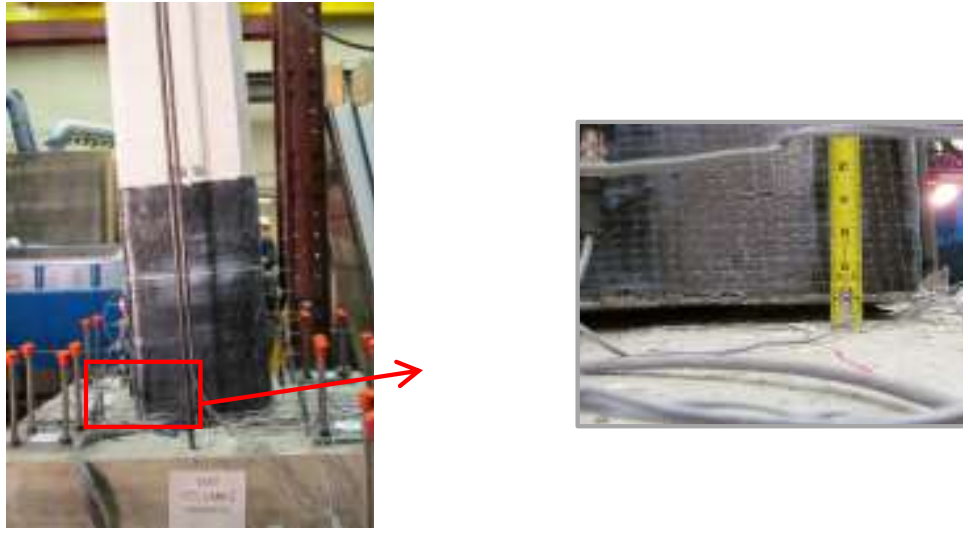

(c)

Figure 4-16 Observed Damage (a) A-C; (b) A-S; (c) R-S 
Furthermore, the contribution of base rotation at the cold joint to the column deformation was dominant for all columns as depicted in Figure 4-17. The base rotation was calculated using the LVDTs located closest to the foundation as illustrated in Figure 4-12 (a). At lateral drift values of $4 \%$ the contributions were approximately $75 \%, 79 \%$ and 78\% for specimen A-C, A-S and R-S, respectively. The incrementally lower contribution from slip to the total drift for the column with a higher axial load is expected as slip displacement decreases with the increase of axial load (Elwood \& Eberhard, 2009).

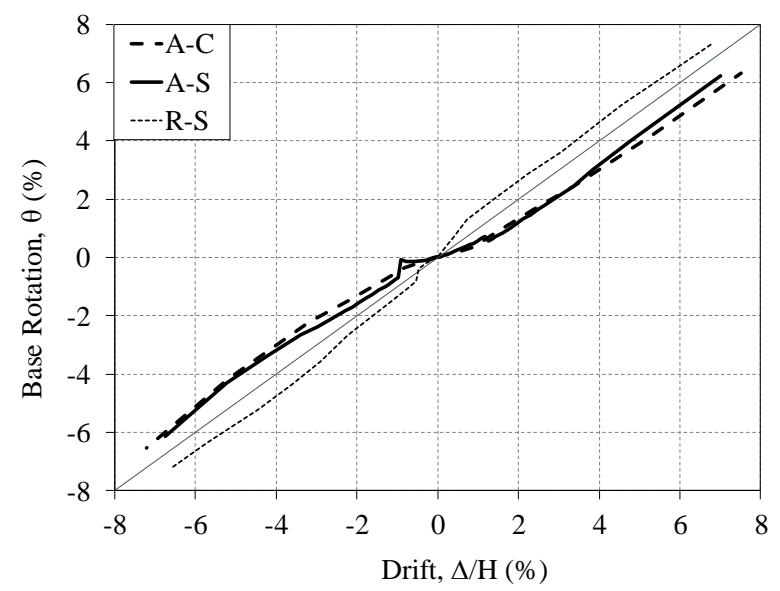

Figure 4-17 Base rotation versus displacement 


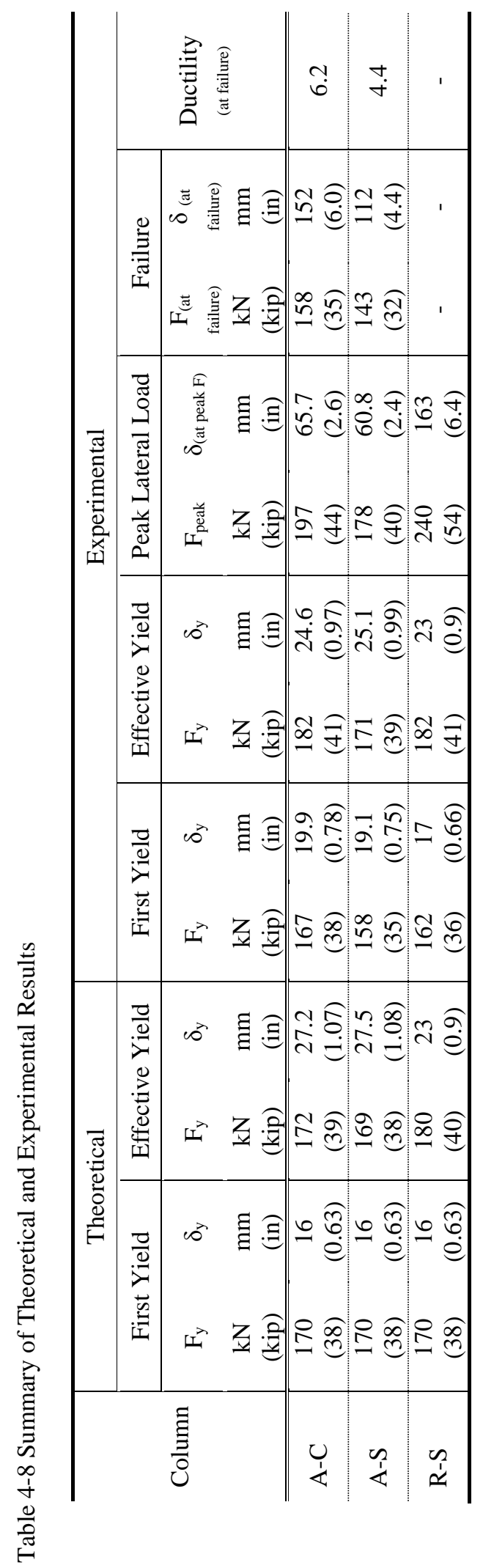




\subsubsection{Backbone Curve Comparison}

To visualize the overall load-displacement of all three specimens, backbone curves were compared among the three experiments and are shown in Figure 4-15 (d). The unretrofitted columns exhibited moderate to high displacement ductility ranging from $\mu=4$ to 6.2 despite the apparent seismic detailing deficiencies whereas the retrofitted column reached ductility greater than 8 . The ductile performance was likely a result of a relatively long lap splice length $\left(28 \mathrm{~d}_{\mathrm{b}}\right)$, a low axial load level $0.07 f^{\prime}{ }_{c} A_{g}$ and a low longitudinal steel reinforcement ratio $(\rho=0.88 \%)$. ElGawady, et al. (2010) also achieved similar results with deficient columns that were flexural dominated. All three specimens had approximately the same initial stiffness.

Comparing columns A-C and A-S underscores the differences in performance caused by the loading protocols. The column subjected to the subduction earthquake based protocol exhibited slightly lower lateral strength by approximately $10 \%$. The strength degradation was greater under the subduction earthquake based protocol, resulting in significantly lower ductility at the point of failure as summarized in Table 4-8. The loading protocols are different in the number of displacement excursion, however the total displacement travelled for both columns at failure was not particularly different and was within $10 \%$. The different performance outcomes can be attributed to the greater number of inelastic cycles that characterize the subduction earthquake based protocol, which is significant given that majority of the cycles are at relatively low ductility values. The increase in the number of inelastic cycles enabled the lap splice failure mode in A-S, which was not prominent in A-C. Additionally, the $0.25 \mathrm{ksi}$ (2.6 
$\mathrm{MPa})$ difference in compressive strength $\left(f^{\prime}{ }_{c}\right)$ at the time of testing may have triggered an earlier formation of the lap splice failure in A-S, however this contribution was likely minor. Column A-S did not develop its full nominal moment strength of $622 \mathrm{kN}-\mathrm{m}$ (5505 kip-in) since the stress in the longitudinal reinforcement did not reach $\mathrm{f}_{\mathrm{y}}$ due to the lap splice, thereby, affecting the lateral load carrying capacity. The effective bar stress $\left(\mathrm{f}_{\mathrm{s}}\right)$ corresponding to the maximum force $\left(\mathrm{T}_{\mathrm{b}}\right)$ that could be transferred through a lap splice can be computed using Equation 4-1 (Priestley, Seible, \& Calvi, 1996).

$$
f_{s}=\frac{T_{b}}{A_{b}}=\frac{f_{t} \cdot p \cdot l_{s}}{A_{b}} \quad \text { Equation } 4-2
$$

Where, $A_{b}$ is the area of the longitudinal rebar, $f_{t}$ is the concrete tension stress, $p$ is the concrete perimeter associated to the failure surface of each bar, and $1_{\mathrm{s}}$ is the length of the splice. Priestley recommended using a conservative value of $f_{t}=0.33 \sqrt{f_{c}{ }^{\prime}} M P a$ based on experimental results, and a perimeter ( $p)$ equal to $2 \sqrt{2}\left(c+d_{b}\right)$ for widely spaced lap-spliced bars, where $\mathrm{c}$ is the concrete cover and $\mathrm{d}_{\mathrm{b}}$ is the diameter of the longitudinal rebar. The effective bar stress in columns A-C, A-S and R-S were computed to be $70 \mathrm{ksi}$ (482 MPa), $68 \mathrm{ksi}\left(469 \mathrm{MPa}\right.$ ) and $71 \mathrm{ksi}$ (490 MPa), respectively, compared to $f_{y}=70$ ksi (482 MPa). Hence, column A-S was not able to develop its flexural strength due to failure of lap splice.

\subsubsection{Measured Curvature and Strain}

The recorded section curvature profiles along the height of the plastic hinge region and above the foundation are plotted in Figure 4-18 for three representative ductility levels. 
The curvature values were determined from the displacements measured by the LVDTs loading protocol can be more directly observed when comparing the relative curvatures at a common ductility. For example, the curvatures were measured to be $0.24 \mathrm{rad} / \mathrm{m}(0.0061$ $\mathrm{rad} / \mathrm{in})$ and $0.30 \mathrm{rad} / \mathrm{m}(0.0075 \mathrm{rad} / \mathrm{in})$ of $\mathrm{A}-\mathrm{C}$ and $\mathrm{A}-\mathrm{S}$ at ductility value of $\mu=4$. The measured curvature at the base of the column was therefore $24 \%$ higher when the subduction earthquake based protocol was applied. This increase was caused by the greater number of cycles and associated deterioration prior to reaching that target ductility under the subduction earthquake based protocol. These measurements further validated the visual observations of bond-slip failures of the column.

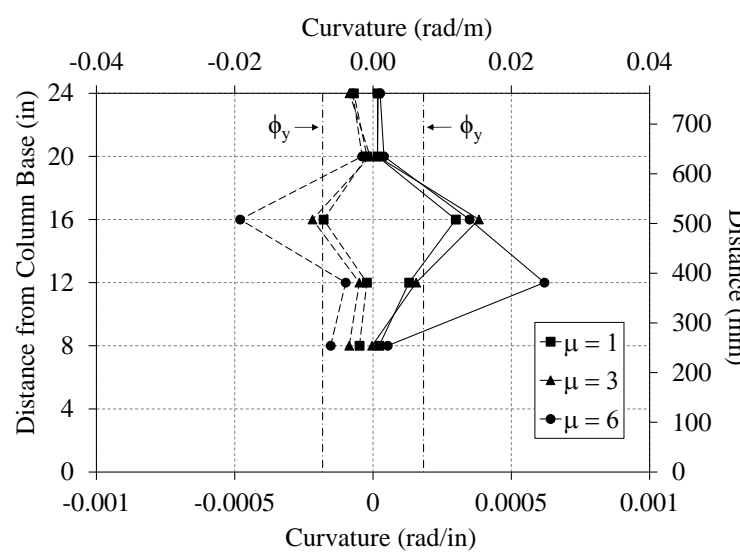

(a)

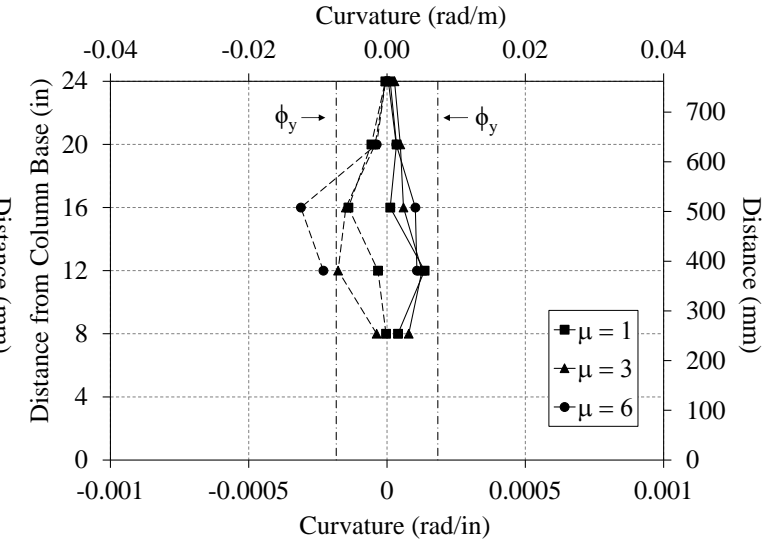

(b)

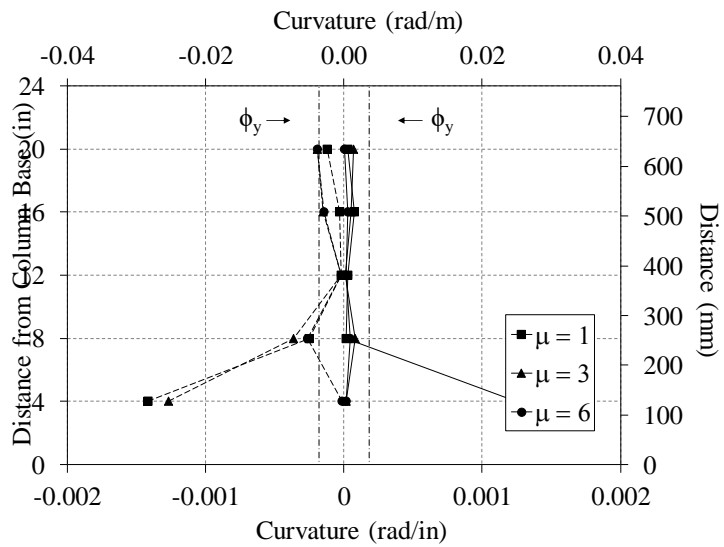

(c)

Figure 4-18 Average curvature profiles (a) A-C, (b) A-S, (c) R-S 
Each column was also instrumented with strain gauges to record the strains imposed during testing of the column. Strain values for dowels were used to plot the strain distribution along the splice length at ductility 1, 2 and 3 in Figure 4-19. The analytical yield strain values were included in the plot using vertical dashed lines for reference. The steel strain distribution along the splice length can be used to evaluate the level of load transferred to the lapped reinforcement. The dowel strains were recorded to have positive values near the top of the footing during negative excursions at ductilities $\mu>1$, whereas the recorded strains above that location remained consistent with the direction of the cyclic excursion. This can be attributed to the rebar not being able to recover the permanent plastic strain experienced near the footing during positive excursions. In these cases the rebar strain was not able to fully compress past yield upon reversal, whereby the localized compression recorded in the curvature measurements may have been accommodated by bar bending. Damage to the specimens ultimately led to damage to the strain gauges making readings for higher ductility levels unreliable.

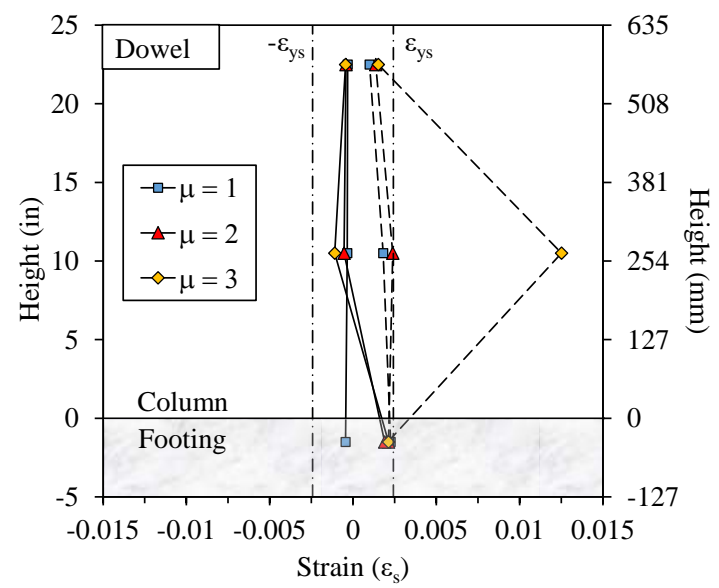

(a)

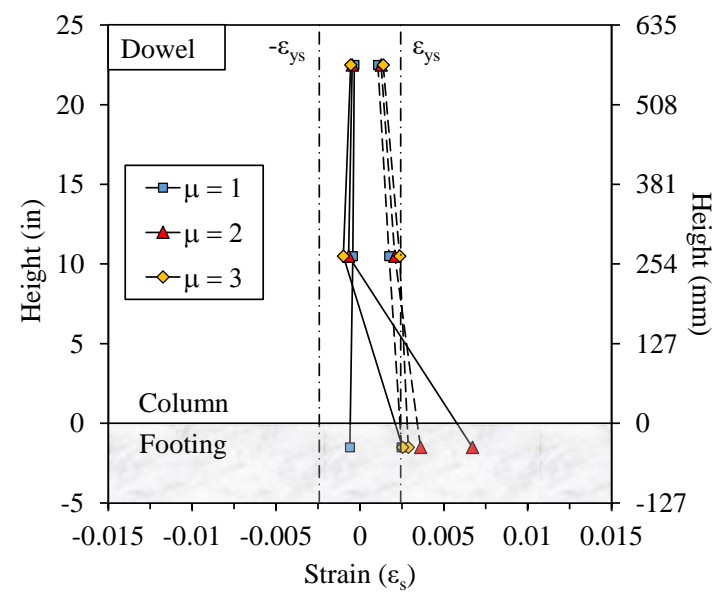

(b) 


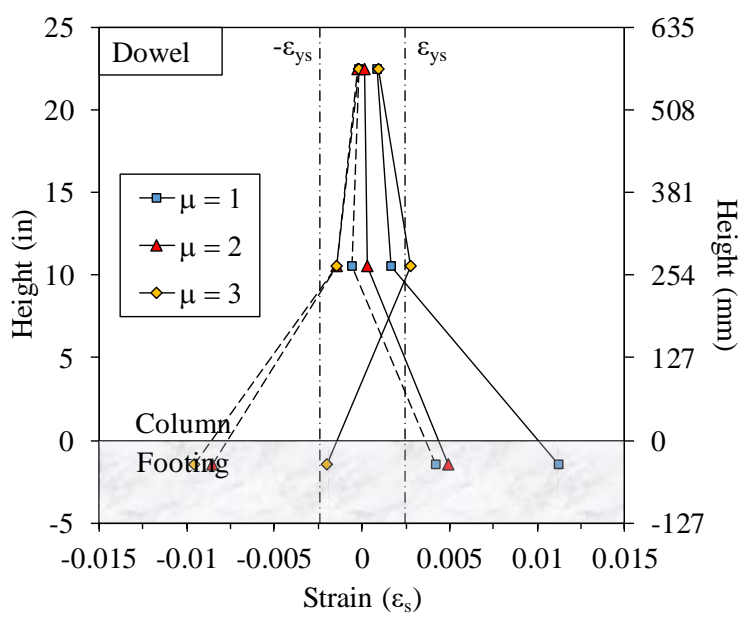

(c)

Figure 4-19 Strain profiles (a) A-C, (b) A-S, (c) R-S

\subsubsection{Stiffness Degradation and Cumulative Energy Dissipation}

Stiffness degradation is a key element for nonlinear modeling of structures. In reinforced concrete structures, stiffness degradation can be attributed to nonlinear behavior of reinforced concrete such as cracking, cover spalling, crushing of concrete, yielding and buckling of reinforcement. In this study, the normalized stiffness, represented as the secant stiffness divided by the yield stiffness, was utilized as the stiffness degradation parameter. Figure 4-20 (a) shows the secant stiffness normalized by the effective yield tangent stiffness versus the corresponding displacement ductility for each specimen. All three test specimens exhibited similar secant stiffness degradation up to a displacement ductility of 2. Column R-S exhibited less degradation compared to the other two columns. Gulkan \& Sozen (1974) proposed a relation between the displacement ductility $(\mu)$ and the secant stiffness $\left(\mathrm{k}_{\mathrm{sec}}\right)$ as $\mathrm{k}_{\mathrm{sec}} / \mathrm{k}_{\mathrm{y}}=1 / \mu \leq 1$. This analytical secant stiffness degradation was also plotted and corresponded closely with the 
experimental data up to displacement ductility of 3 for all three columns. Column A-S began to exhibit a higher stiffness degradation at ductility 4. Consequently, this analytical relationship is appropriate to predict the degradation in the stiffness of reinforced concrete columns regardless of the loading protocol, but may start to underestimate the stiffness when subjected to subduction earthquake based protocols as the column approaches failure.

The energy dissipation was calculated by using the area inside the hysteretic loops. Figure 4-20 (b) shows the normalized cumulative energy dissipation versus displacement ductility achieved for all three columns until failure. The calculated cumulative energy dissipation was normalized by the total dissipated energy at the first yield point $\left(\mathrm{E}_{\mathrm{y}}\right)$. It may be observed that the energy dissipation has improved substantially due to retrofit. The cumulative energy dissipation is a function of not only the column hysteretic behavior, but also the characteristic of the protocols used. The effect of the subduction earthquake based loading protocol can be observed when comparing the dissipated energy of column A-S as compared to A-C. Since the subduction earthquake based protocol consists of steadily increasing ductility cycles, the cumulative energy also steadily increased. The conventional loading protocol on the other hand has larger increments of ductility between cycles and also has repeating cycles at any given ductility level, resulting in abrupt changes in the cumulative energy dissipated. At approximately ductility $\mu=4$, nearly the same cumulative energy dissipation was achieved between columns A-S and A-C. Yet, column A-S failed shortly thereafter, while column A-C continued to reach higher levels of ductility and continued to dissipate 
energy, reaching twice as much cumulative energy at failure. So despite the differences in ductility capacity of the columns, the column subjected to the subduction earthquake based protocol did not accumulate more dissipated energy.

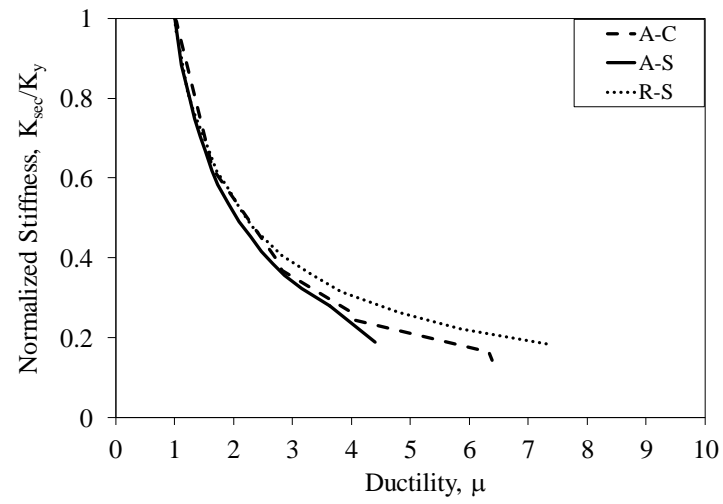

(a)

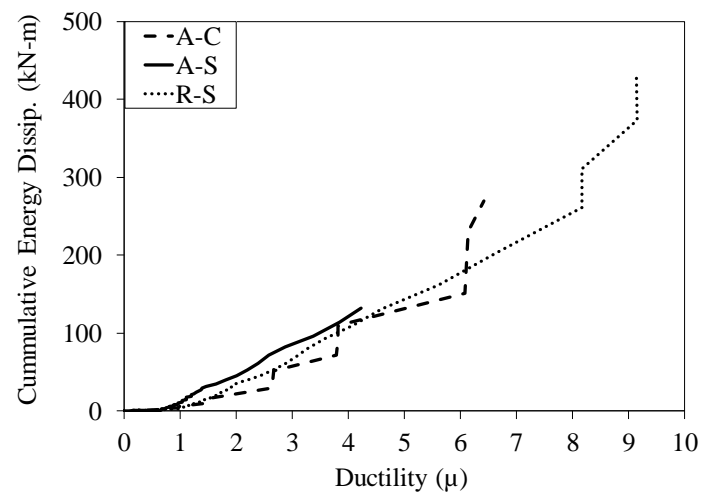

(b)

Figure 4-20 (a) Stiffness degradation; (b) cumulative energy dissipation

\subsubsection{Numerical Model}

Distributed-plasticity fiber-section modeling have been extensively used to characterize the behavior of RC columns (Taucer, Spacone, \& Filippou, 1991), (Scott \& Fenves, 2006), (Berry \& Eberhard, 2008). In the distributed-plasticity method, the nonlinear behavior is distributed along the length of the member through the use of discrete fiber sections and integration points. The RC bridge columns presented in this paper were modeled using this methodology with the aim of providing modeling parameters and assess the effect of the loading protocol on the overall column behavior. OpenSees (2013) was utilized to carry out the numerical modeling for its vast library of available materials and elements to characterize the nonlinear response of structural components. 
The model used in this study is depicted in Figure 4-21. Four nodes were used to represent the column test model. The bottom node represents the interface between the $\mathrm{RC}$ column and the footing. This node was constrained in all directions in order to represent a fixed support. The adjacent node was used in order to include the bond-slip contribution. The axial load, $\mathrm{P}$, and the lateral load, F, were applied at the very top node at a height of $2870 \mathrm{~mm}$ (113 in) in order to mimic the actual test setup, where the axial and the lateral load were directly applied to the steel beam located on top of the columns. Further, the transfer steel beam was represented in the model through the use of an elastic element of length $330 \mathrm{~mm}$ (13 in). The very top node was selected as the control node to record the lateral displacement of the column.

In the distributed-plasticity model, discrete fiber sections were used throughout the length of the column. A force-based beam-column element with six integration points was used to represent the column. The number of integration points was selected following the recommendation of Berry and Eberhard (2008). The Gauss-Lobatto integration rule was selected as the plastic hinge integration method. This integration rule includes additional integration points at the element ends.

The column section consists of three types of fiber representing the core (confined) concrete, cover (unconfined) concrete and the reinforcing steel as shown in Fig. 9. The cover and core concrete were discretized into 24 and 20 strips in both directions, respectively. The Concrete 02 with linear tension softening uniaxial material was used to model both confined and unconfined concrete. In order to obtain the confined concrete parameters, the model proposed by Mander et al. (1988) was used. The 
longitudinal reinforcing steel was modeled using the uniaxial hysteretic material with the stress-strain values for the reinforcing steel shown in Table 1, which represent the measured values from tensile testing of coupons. The Hysteretic material was selected because it can capture pinching of force and deformation, which is important to characterize bond-slip failure in RC columns with lap splices. The parameters pinchx and pinchy were set equal to 1.0 in order to represent the observed pinching behavior of the columns. The hysteretic material is also capable of representing damage due to ductility and energy, and degraded unloading stiffness that can be caused by concrete crushing, splitting cracks, bar buckling and bar fracture. The damage parameter damage1, which is related to the damage due to ductility was set equal to 0.006 to account for the cyclic deterioration from one cycle to the following cycle observed in the columns. The parameter damage2, which takes into account the damage due to energy, was set equal to 0.002 in order to account for strength and stiffness deterioration observed at a fixed displacement or strain amplitude. The degraded unloading stiffness parameter (beta) was set equal to 0.3 for all columns.

The numerical model was able to characterize the behavior of the tested columns. Comparisons between the experimental and the numerical results using the distributedplasticity approach are shown in Figure 4-22. Initial stiffness, strength and deformation capacities, pinching effect, and strength and stiffness degradation were closely represented by the model. This distributed-plasticity model strategy may be used in cases of biaxial loads without major modifications, making it suitable for three dimensional modeling cases. 
Hysteretic cyclic deterioration plays a fundamental role in the assessment of bridge columns subjected to ground motions. In the case of the distributed-plasticity model, values of 0.006 and 0.002 were proposed to account for the deterioration due to ductility (damage1) and energy (damage2), respectively. These values are recommended for use in predictive analysis of substandard RC bridge columns. (Mehary, Dusicka, \& Bazaez, 2017)

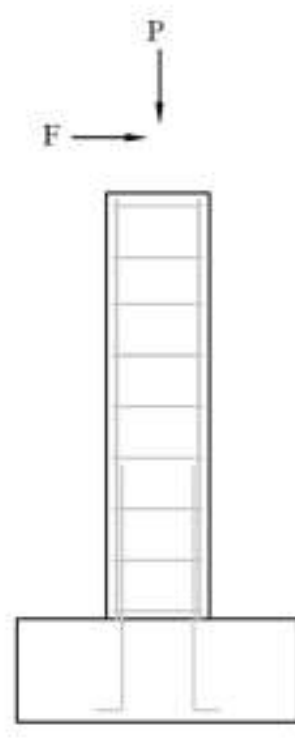

RC Column

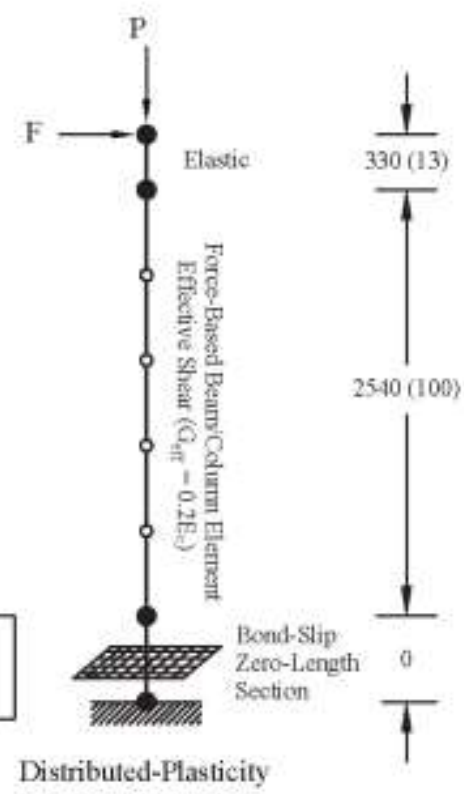

Figure 4-21 Numerical model.
- Node

- Integration Point

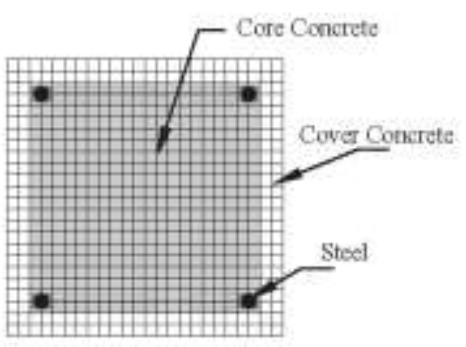

Column Fiber Section 


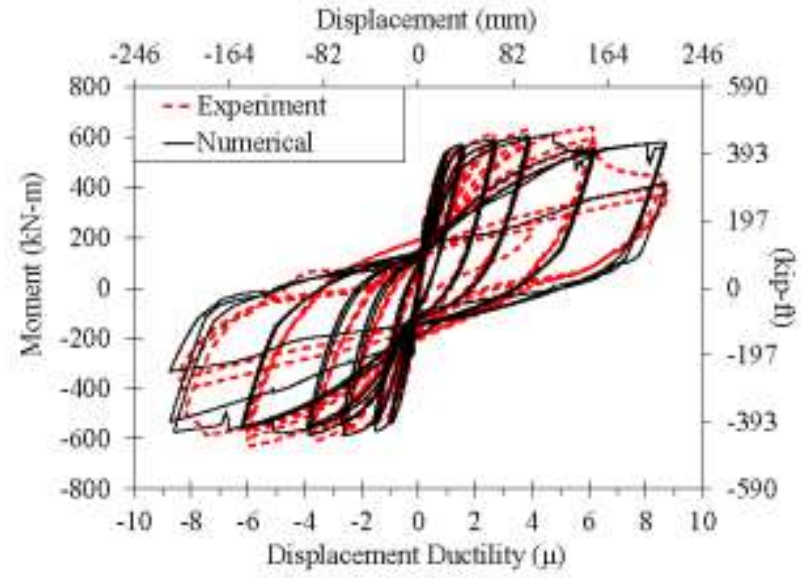

(a)

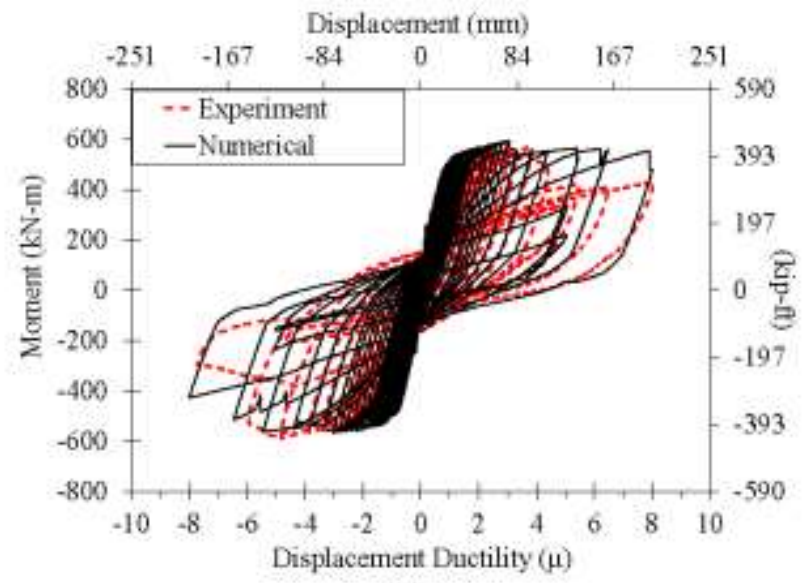

(b)

Figure 4-22 Experimental vs numerical results. (a) A-C, (b) A-S 


\subsection{Summary}

Full-scale reinforced concrete square cross-section bridge columns, which were designed to represent columns in multi-column bents constructed pre-1970s in the Pacific Northwest, were experimentally evaluated to determine their cyclic behavior. Two types of loading protocols were utilized, a conventional cyclic loading protocol and a cyclic loading protocol that aims to assess the behavior of bridge columns under subduction zone earthquakes. Numerical models were developed to evaluate the damage parameters needed to effectively capture the cyclic behavior. Based on the results, the following observations and conclusions were made:

- The experimental results indicate that despite substandard detailing, the flexure-dominated square columns in bridges built before 1970 in the Pacific Northwest can exhibit moderately ductile performance, a result of a relatively long lap splice length $\left(28 \mathrm{~d}_{\mathrm{b}}\right)$, a low axial load ratio $\left(0.07-0.17 \mathrm{f}_{\mathrm{c}} \mathrm{A}_{\mathrm{g}}\right)$ and a low longitudinal steel reinforcement ratio $(\rho=0.88 \%)$.

- Despite the surprising moderately ductile performance of the as-built, the CFRP retrofit considerably enhanced displacement ductility. The retrofit helped inhibit bond failures in lap splices of longitudinal reinforcement in the plastic hinge regions and provided confinement producing very minimal spalling of concrete at the base of the column. Hence, retrofit designs targeting different ductility levels could take advantage of the recorded behavior to select a cost effective retrofit measure. 
- The column displacement ductility can be adversely affected when subduction earthquake based loading protocol is considered. While the specimen tested with conventional loading protocol reached a displacement ductility of 6.2 , a nominally identical specimen tested with subduction earthquake based protocol reached a displacement ductility of 4.4 due to higher levels of strength degradation.

- The experiments also demonstrated that subduction earthquake based loading protocols may change the observed failure mode of columns with lap splice in the plastic hinge region. The primary mode of failure for the column tested under the conventional cyclic loading protocol was flexural tension failure causing crushing of concrete at the base of the column, causing plastic behavior to be concentrated at the base at the cold joint between footing and column. The primary mode of failure for the column tested under the subduction loading protocol was by lap splice failure, highlighted by vertical cracks at the splice location and higher bond-slip deformations. This change in failure mode can be attributed to the presence of many more inelastic lowamplitude, yet damaging, cycles that characterize subduction earthquakes 


\section{CHAPTER 5}

\section{SEISMIC FRAGILITY ASSESSMENT}

\subsection{General}

Bridge fragility curves describe the probability of a bridge reaching a certain damage state for a given ground motion parameter, hence, play a very important role in the seismic risk assessment of a transportation network. A fragility function is a conditional probability that gives the likelihood that a structure will meet or exceed a specified level of damage for a given ground motion intensity measure and can be expressed as a conditional probability that a defined limit state (LS) is exceeded for a given level of ground motion intensity (IM). This conditional probability is given in Equation 5-2. A graphical representation of this function is given by Figure 5-1.

$$
\text { Fragility }=\mathrm{P}[\mathrm{LS} / \mathrm{IM}=\mathrm{y}] \quad \text { Equation } 5-1
$$

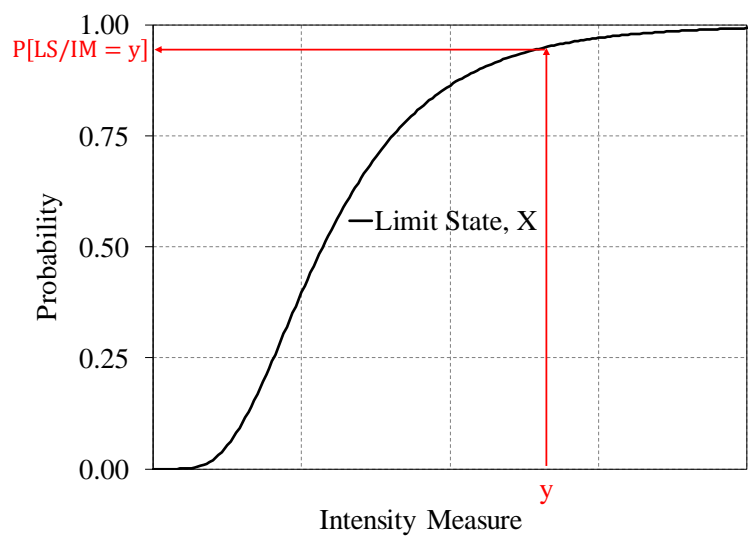

Figure 5-1 Fragility Curve Example

Earthquake loss methodologies that are integrated with a geographic information system have been used in large-scale simulations of transportation networks. Several 
projects, such as 1999 HAZUS software (Basoz \& Mander, 1999), associated PEER highway demonstration research (Moore, Cho, Fan, \& Werner, 2006), and REDARS (Werner S. D., et al., 2006) have modeled transportation networks to assess the economic impact analysis of damage caused by an earthquake event to a transportation network. The hazard assessment components of these methodologies follow well-known hazard analysis methods and rely on application tools developed in HAZUS (1999). Damage to bridges in the highway network contributes significantly to both direct and indirect losses. Bridge damage fragility curves describe the conditional probability of exceeding a level of direct or indirect bridge damage for a given level of seismic hazard.

Currently, limited bridge fragility has been developed for retrofitted bridges. Fragility curves that have been developed for retrofitted bridges have been primarily based on numerical analyses of California type bridges (Shinozuka, Kim, Kushiyama, \& Yi, 2002) or on bridge types typical to the eastern part of the country (Padgett \& DesRoches, 2008). In order to have more relevant results for Oregon and in order to assess the effectiveness of a retrofit, fragility relationships relevant to the Oregon bridge inventory are needed.

In this chapter, the various ways bridge fragility curve can be developed are presented and compared. Following, fragility curves for a representative bridge bent in its as built and retrofitted conditions are developed using analytical methods using actual subduction zone strong motion records in conjunction with nonlinear analyses in SAP2000. 


\subsection{Fragility Development Methods}

Fragility curves can be developed by using empirical methods, expert opinions, or through analytical methods. Empirical fragility curves are developed by utilizing observed damage data during past earthquakes, and fragility curves based on expert opinion are very subjective and area based on limited damage data in defining damage state which makes this method not practical in developing fragility curves for retrofitted bridges (Padgett \& DesRoches, 2008). Analytical fragility curves, on the other hand, are developed by numerical simulations of the response of a structural model of a particular type of bridge subject to earthquake ground motions. Analytical fragility functions can be elastic spectral response, non-linear static analysis, or non-linear time history analysis. An overview of the literature on the above mentioned methods is provided below.

\subsubsection{Empirical Methods}

Empirical fragility curves are developed utilizing bridge damage data obtained from past earthquakes particularly in the 1989 Loma Prieta, 1994 Northridge, and 1995 Kobe earthquakes. Empirical fragility development method is relatively straightforward compared to the other methods. Fragility curves are expressed in the form of twoparameter lognormal distribution functions of peak ground acceleration to represent the intensity of the seismic ground motion. Earthquake damage reports are used to establish the relationship between the ground motion intensity and the damage state of each bridge.

Basoz \& Kiremidjian (1998) conducted studies to develop bridge fragility curves by statistically analyzing empirical damage data from the damage reports of the Loma Prieta (1989) and Northridge (1994) earthquakes. They compiled structural 
characteristics for the groups of bridges that were exposed to ground shaking such as abutment type, number of spans, type of superstructure and substructure, length and width of the bridge, skew, etc. They also compiled detailed damage descriptions and the corresponding damage states for bridges damaged in the two earthquakes. Since there were no guidelines for evaluating bridge damage states, they proposed definitions for damage states for columns, abutments, and joints and connections for concrete bridges based on the observed bridge damage in the Northridge earthquake.

Yamazaki et al. (1999) proposed fragility curves for expressway structures in Japan based on actual damage data from the 1995 Kobe earthquake. They estimated spatial distribution of earthquake ground motion indices by using kriging technique, which is a method of stochastic interpolation. They then compared actual data of damage to the expressway network to the estimated ground motion indices and constructed fragility curves with lognormal distributions. Shinozuka, et al. (2000) considered both empirical and analytical fragility curves. They utilized bridge damage data obtained from the 1994 Northridge and 1995 Kobe earthquake to develop empirical fragility curves. They made use of the damage reports to establish the relationship between the ground motion intensity and damage state of each bridge. In order to estimate the parameters of the lognormal distribution function they carried out two different methods. In method 1, they developed a fragility curve for each damage state independently for each bridge sample with a given set of attributes. This method assumed that the entire sample was statistically homogeneous. Method 2, they estimated the parameters of lognormal distribution function representing different damage states simultaneously by means of maximum likelihood method. Here the parameters estimated were the medians of each 
fragility curve and one value of the log-standard derivation arranged to be common to all the fragility curves.

\subsubsection{Expert Opinion}

Expert opinions are collected to estimate structural damages from earthquakes. The survey results are then represented in a damage probability matrix describing damage state for different levels of ground motion usually using the Modified Mercalli Intensity (MMI) scale. Damage curves are then developed from the probability matrix.

ATC (1991) performed a survey to gather expert opinions to estimate structural damage. They had only five bridge experts that responded and offered their opinion on bridge damages. The survey results were represented in a damage probability matrix that described probabilities of specific damage states for different levels of ground shaking using the Modified Mercalli Intensity (MMI) scale. Based on the damage probability matrix, damage curves were developed.

Grossi (2000) gathered information from experts on structural vulnerability and the benefits of retrofitting a structure for an earthquake hazard and then proposed a procedure for conversion of this data from mean damage at various levels of ground shaking to a fragility curve. Grossi first aggregated expert opinion data on mean damage versus MMI into an analytical distribution of each level of ground shaking. The distribution was then utilized to develop damage probability matrices (DPM). Once the probabilities were developed, a cumulative lognormal distribution function was fit to these cumulative DPM data points. The MMI was then converted to PGA, which in turn was converted to spectral acceleration $\left(\mathrm{S}_{\mathrm{A}}\right)$ and spectral displacement $\left(\mathrm{S}_{\mathrm{D}}\right)$ pairs based on 
the capacity spectrum method (CSM). The CSM is used to estimate the peak building response at the intersection of the building capacity curve and the response spectral displacement demand.

\subsubsection{Analytical Methods}

\subsubsection{Elastic Spectral Analysis}

Hwang et al. (1999) (2000) (2001) proposed an analytical method to develop fragility curves for bridges in the Memphis area. They first established bridge models for each bridge type and then evaluated bridge components that had potential for being damaged during an earthquake to determine their capacity to damage ratios. They determined capacity as described in the Seismic Retrofit Manual for Highway Bridges and seismic damage from elastic spectral analysis according to the method specified in the Standard Specifications for Highway Bridges (AASHTO, 1996). There were three damage states considered in their study, namely, no/minor damage, repairable damage and significant damage. For each level of PGA they performed 50 calculations of bridge damage states. The bridge damage data were then statistically analyzed and the results displayed as fragility curves.

\subsubsection{Non-Linear Static Analysis}

Mander \& Basöz (1999) developed a theoretical basis of establishing fragility curves for highway bridges through the use of rapid analysis procedures from the fundamentals of mechanics and dynamics. They used three sources of data for the analysis, that is National Bridge Inventory (NBI) records that contain the bridge attributes 
and geographical location, ground motion data (usually best obtained from the USGS web site), and geological maps. They used a capacity-spectrum approach to develop the fragility curves for bridges. They validated the analytically predicted values against fragility curves that were empirically derived from data gathered for bridges damaged in the 1994 Northridge and 1989 Loma Prieta earthquakes. These fragility curves developed by Mander and Basöz were used in a geographic information system-based natural hazard loss estimation software package developed and freely distributed by the Federal Emergency Management Agency (FEMA) called HAZUS. Two types of bridge fragility curves are used in HAZUS. One is based on the peak ground acceleration (PGA) and the second is based on the permanent ground displacement (PGD).

Shinozuka, Feng and Kim, et al (2000) examined fragility curves of a bridge by time history analysis and the capacity spectrum method which is one of the simplified nonlinear static procedures developed for buildings. To determine capacity they performed pushover analysis. The force-displacement curve was plotted by tracking the total shear force at the column bases as a function of displacement of the superstructure. The lateral forces were applied in proportion to the fundamental mode shape from the capacity spectrum method. Their comparison of fragility curves generated by the nonlinear static procedure with those by time-history analysis indicated that there was good agreement for the state of minor damage, but not as good for the state of major damage where nonlinear effects clearly played a vital role.

Liao and Loh (2004) used a nonlinear static analysis method for the development of analytical fragility curves in terms of PGA. The demand spectrum is given in terms of 
site amplification coefficients and demand spectral acceleration for different ranges of periods. The demand spectral acceleration at period $\mathrm{T}$ second is obtained by first specifying attributes of the scenario earthquake including location, depth, magnitude, fault rapture type, etc. Then the ground motion levels for the bedrock using appropriate attenuation laws are determined. Site amplification factors are included for short and long periods. The bridge capacity curve is evaluated by first creating a computer model of the prototype bridge structure. This capacity is used to demonstrate the seismic capacity of a bridge structure. The ordinate gives the shear and abscissa is the displacement. Then a nonlinear pushover test, which is the relationship between the base shear and the displacement, can be established and convert the pushover curve to a capacity spectrum by using empirical equations. They used the fragility curves generated for seismic loss estimation for the transportation system.

\subsubsection{Non-Linear Time History Analysis: Synthetic Ground Motion Sources}

Shinozuka, Feng and Lee, et al (2000) constructed analytical fragility curves on the basis of the nonlinear time history analysis. They used two representative bridges with a precast, prestressed continuous deck in the Memphis area. For the seismic ground motion, they used time histories generated by the Center for Earthquake Research and Information at the University of Memphis. They randomly selected 10 time histories from the 50 histories generated for different combinations of magnitude and epicentral distance. They used SAP2000 finite-element code to approximately simulate the state of damage of each bridge under a ground acceleration time history. The median and log- 
standard deviation parameters for the log-normal fragility curve were estimated by maximum likelihood procedure and the fragility curves are generated.

Hwang, Liu and Chiu (2001) presented an analytical method for the development of fragility curves of highway bridges. They first created a suitable model of the bridge of interest. They then generated a set of synthetic acceleration time histories that covered a range of ground shaking strength. A nonlinear time history response analysis was then performed for each earthquake-site-bridge sample to simulate a set of bridge response data. A regression analysis was then performed of the simulated response data to set up the probabilistic characteristics of structural demand as a function of a ground shaking. Once the bridge damage states were defined and the probabilistic characteristics of structural capacity corresponding to each damage state established, the conditional probabilities that structural demand exceeds structural capacity for various levels of ground shaking were computed. The fragility curves were then finally plotted as a function of the ground shaking parameter.

Shinozuka, Saxena, et al. (2001) studied the impact of stochastic spatial variability of seismic ground motion on the seismic response of long, multi-span, reinforced concrete bridges. They selected a twelve-span continuous reinforced concrete bridge. They analyzed the bridge with SAP2000 finite element program. They generated the seismic ground motion time histories that were compatible with prescribed response spectra at the supports of the bridge and to reflect a prescribed coherence function, apparent velocity of wave propagation and duration of strong ground motion. They defined five different damage states in terms of ductility demand of the piers. The 
fragility curves were expressed in the form of two-parameter lognormal distribution functions where the two parameters were estimated by the Maximum Likelihood Method treating each event of bridge damage as a realization from a Bernoulli experiment.

Choi Et al. (2003) developed analytical fragility curves that were based on nonlinear response history analyses and a suite of synthetic ground motions. They represented the bridge by an analytical model that included the inelastic behavior of the appropriate components. They then developed earthquake input motion for various characteristic magnitudes, epicentral distances, and local soil conditions. After establishing a set of earthquake-bridge sample where the uncertainties in seismic source, they quantified path attenuation, soil conditions and bridge components. They then performed a non-linear response history analysis on these samples. They defined damage states for column ductility demand, steel fixed and expansion bearing deformations, and elastomeric bearing deformations. And by using these predetermined damage indices, they assigned a damage state to each component of the bridge, which were combined into fragility curves that represented the entire bridge system using first-order reliability principles.

Y. Pan (2007) developed seismic fragility curves as a function of moment magnitude and epicentral distance for individual bridge components and entire bridge systems, and investigated the effectiveness of different seismic retrofit approaches in reducing seismic fragility. Pan developed three-dimensional finite element models for two typical New York State bridge types, namely, multi-span continuous and multi-span simply supported steel bridges using SAP2000 (9.1.1.V). Pan then performed nonlinear 
time history analyses of 10 bridge samples for both typical bridges using 100 simulated earthquake ground motions where each bridge sample was subjected to 10 different ground motions selected to cover a wide range of PGAs. She gathered maximum response quantities of bridge components and estimated capacities of bridge samples at various damage states for each of the 100 analysis cases. She then carried out linear and quadratic regressions between the logarithm of demand to capacity ratio, and $\ln$ (PGA). Once the mean and standard deviation were obtained through regression analysis, fragility curves were developed as a function of PGA.

Nielson and DesRoches (2007) generated analytical fragility curves for typical bridges in the Central and Southern United States. They developed seismic fragility curves for nine classes of bridges (common three-span, zero-skew bridges with nonintegral abutments). The methodology they adopted used 3-D analytical models in combination with a suite of 96 synthetic ground motions and nonlinear time-history analyses. An important aspect of the methodology they selected was that it considered the contribution of multiple bridge components. Their results showed that multi-span steel girder bridges were the most vulnerable of the considered bridge classes while singlespan bridges tended to be the least vulnerable. They made a comparison of their proposed fragility curves with the ones that are currently found in HAZUS-MH and they determined that it showed a strong agreement for the multi-span simply supported steel girder bridge class. However, for other simply supported bridge classes (concrete girder, slab), the proposed fragility curves suggested a lower vulnerability level than what the ones in HAZUS-MH suggest. 


\subsubsection{Non-Linear Time History Analysis: Strong Ground Motion from Past Records}

Karim and Yamazaki (2000) constructed fragility curves for bridge piers of specific RC bridges in Japan and compared the results with the empirically developed fragility curves proposed by Yamazaki et al. (1999). They first selected strong motion records for the 1995 Kobe earthquake. Fifty time histories were taken on the basis of Peak Ground Acceleration (PGA) and Peak Ground Velocity (PGV). They then used this acceleration time histories as input time ground motion and obtained bridge damage indices for the bridge piers. Finally using the damage and ground motion indices, they constructed fragility curves for RC bridge piers. The comparison of the fragility curves they obtained were in agreement with those developed by empirical methods Yamazaki et al. (1999).

Karim and Yamazaki (2001) considered an analytical approach to construct the fragility curves for bridge piers of specific bridges designed using the 1964 and 1998 seismic design codes for highway bridges in Japan. For a non-linear dynamic response analysis they selected earthquake ground motion records on the basis of large peak ground acceleration (PGA) from the 1995 Hyogoken-Nanbu (Kobe), the 1994 Northridge, the 1993 Kushiro-Oki and the 1987 Chibaken-Toho-Oki earthquakes. They considered a total of 50 acceleration time histories from each earthquake event. After normalizing the PGA of the selected earthquakes to different excitation levels, they created an analytical model of the bridge and obtained the stiffness of the structure. They then selected a hysteretic model for the non-linear dynamic response analysis and carried out the non-linear dynamic response analysis using the selected records. The ductility 
factors of the structures were then obtained and the damage indices of the structure for each level of excitation were also obtained. Finally, using the damage indices and the ground motion indices, the analytical fragility curves were constructed.

Karim and Yamazaki (2003) adopted an analytical approach to construct fragility curves for highway bridge piers. Four typical RC bridge piers and two RC bridge structures were considered, of which one was a non-isolated system and the other was an isolated system. The bridges were designed according to the seismic design code in Japan. The researchers used strong motion records from Japan and the United States, and performed non-linear dynamic response analyses and obtained the damage indices for the bridge piers. The fragility curves for the bridge piers were constructed using the damage indices and ground motion indices assuming a lognormal distribution. They also obtained a relationship between the fragility curve parameters and the over-strength ratio of the structures by performing a linear regression analysis. They observed that the fragility curve parameters showed a strong correlation with the over-strength ratio of the structures.

Simon, Bracci and Gardoni (2010) examined the effects of reinforcement corrosion on strength due to a reduction of reinforcement area, on stiffness due to spalling of the concrete cover, and on seismic fragility. For the analysis they employed both nonlinear static and dynamic analyses. The nonlinear, time-history dynamic analyses were performed to determine the seismic response of the bridge structure to a group of varying intensity earthquake records. A suite of earthquake records constituting a representative sample of expected seismic activity for the bridge were used as earthquake 
ground motion. They chose six earthquakes from the Pacific Earthquake Engineering Research Strong Motion Catalog. They then compared the six chosen earthquakes' median spectral acceleration to the theoretical spectra determined using the attenuation law in Abrahamson and Silva (1997). They studies the inelastic behavior of the bridge by creating additional bins by scaling up the six records already selected. The seismic demand model developed predicts the maximum total drift based on the spectral accelerations corresponding to the fundamental periods in the longitudinal and transverse directions. The mean drift capacities of 1,2 and 4\% for insignificant damage (immediate occupancy or serviceability), moderate damage (life safety or damage control), and severe damage (collapse prevention), respectively, and defined collapse as the drift corresponding to a decrease in lateral resistance of $20 \%$ compared to peak value are then used to define capacity of bridge. Simon et al then developed a probabilistic seismic demand model, which is then combined with the capacity model and commonly accepted capacity limit states to determine and compare the seismic fragility for different levels of corrosion damage.

\subsubsection{Other methods}

Stojadinović and Mackie (2007) developed fragility curves based on the bridge force reduction factors (R-factors) for three damage states, namely concrete cover spalling, longitudinal bar buckling, and column failure. They used sophisticated finite element models and nonlinear time history analyses to define demand and they defined capacities in terms of actual damage states rather than discrete demand levels. The Rfactor parameterized damage fragility curves accounted for the structural characteristics 
of each particular bridge through the use of the bridge force reduction factor parameter unlike those provided by HAZUS that utilize the same damage fragility curve for all instances within a class of bridges.

\subsection{Fragility Curves for Retrofitted Bridges}

Shinozuka, Kim, et al (2002) developed fragility curves for two sample bridges in Southern California strengthened for seismic retrofit by means of steel jacketing of columns. They used SAP2000 nonlinear finite element computer code for twodimensional response analysis of the bridge under sixty Los Angeles earthquake time histories, which were developed for the FEMA SAC steel project, to develop fragility curves before and after column retrofit.

\section{J. Padgett (2005) reviewed bridge seismic retrofit practice in the Central and} Southeastern United States, along with the current state of the art in bridge fragility curve development with the intention to establish a framework for further investigation of the development of analytical fragility curves for bridges in their retrofitted conditions. Padgett developed an analytical, two-dimensional nonlinear model that was modified to incorporate elements for the retrofits at the locations of the bridge using the Open System for Earthquake Engineering Simulation (OpenSees) computational analysis program.

Each bridge sample was matched with 48 earthquake samples from the suite of ground motions that were randomly selected from each magnitude and distance combination. Padgett then performed a non-linear time history analysis for each of the earthquakebridge samples while keeping an eye on the maximum quantities of interest for the components. Once the median values of seismic demand as a function of ground motion 
intensity were determined, the structural capacity or structural limit states with a descriptive damage state were determined. Padgett finally determined the fragility of a structural system or structural component by the probability that the structural demand exceeded the structural capacity. The fragility was modeled by a lognormal cumulative distribution function where the structural demand and capacity were assumed to be lognormally distributed.

Padgett and DesRoches (2007) evaluated the modeling parameters which significantly affect the seismic response of an example class of retrofitted bridges. They developed three-dimensional analytical models in OpenSees and used them in time history analyses to simulate the seismic response of the retrofitted bridges. They also developed retrofit models with an emphasis on representing potential nonlinear behavior of the retrofitted component. They used two suites of synthetic ground motions for the central and southeastern United States (CSUS) for their analysis. Their study showed that the seismic responses of various components in the retrofitted bridges were sensitive to a number of different modeling parameters. They found the fragility to be particularly sensitive to the spread of uncertainty in the base geometry span length, column height, deck width which is inherent to vulnerability assessments for structural portfolios.

Padgett and DesRoches (2007) (2008) (2009) presented an analytical methodology for developing fragility curves for classes of retrofitted bridge systems. Since there were no records of strong motions for the Central and Southeastern U.S., they used two suites of synthetic ground motions for the study. The scenario ground motions were developed based on stochastic methods, considering non-linear site response, and 
the influence of the deep soil column of the upper Mississippi embayment. Open System for Earthquake Engineering Simulation (OpenSees) was used to create a 2D model of the bridge for the fragility analysis.

Billah, Alam, \& Bhuiyan (2013) did seismic vulnerability assessment of retrofitted multicolumn bridge bents and generated fragility functions using a probabilistic seismic demand model. The four retrofit techniques were carbon fiberreinforced polymer (CFRP) jacketing, steel jacketing, concrete jacketing, and engineered cementitious composite (ECC) jacketing. Their results indicate that bridge bents retrofitted with CFRP and ECC have less vulnerability at the different damage states.

\subsection{Fragility Curve for a Representative Deficient Three-Span Concrete Continuous}

\section{Girder Bridge}

In this study, the seismic fragility curves for a representative concrete bridge built pre-1970s in Oregon, were developed using SAP2000 and Capacity Spectrum method proposed by Shinozuka et al. (2000). Bazaez \& Dusicka (2018) developed fragility curves that are Oregon specific for multi-span continuous concrete bridges in their asbuilt state. They utilized ground-motion time histories from Tohoku earthquake M9.0, Maule, Chile earthquake M8.8, and Valparaiso, Chile earthquake M7.8 and selected 10 ground-motion time histories from each earthquake record. The damage states were chosen based on strain and stress levels obtained from experimental studies. In their work, Bazaez and Dusicka (2018) used displacement ductility, $\mu$, as the engineering demand parameter (EDP) to define damage state of the columns. The values are based on 
results from experiments and criteria defined in a study done by Bazaez et al. (2015) and Hwang et al. (2001). These values are given in Table 5-1.

Table 5-1 Damage states for fragility curve development (Bazaez et al. 2015)

\begin{tabular}{c|c|c|c|c}
\hline \multirow{2}{*}{ EDP } & \multicolumn{4}{|c}{ Damage States } \\
\cline { 2 - 5 } & Slight & Moderate & Extensive & Collapse \\
\hline \hline $\begin{array}{c}\text { Displacement ductility } \\
(\mu)\end{array}$ & $\mu>1$ & $\mu>1.4$ & $\mu>2$ & $\mu>4.5$ \\
\hline
\end{tabular}

\subsubsection{Bridge Model Description}

A description of the MSCG bridge class is obtained through a detailed analysis of the National Bridge Inventory (NBI) data base. In addition, specific details of the various bridge components are collected from available bridge plans. The basic bridge configuration used in this study is shown in Figure 5-2.

The superstructure of MSCG bridges consists a deck with asphalt wearing surface, parapets, and diaphragms on top of continuous girders. In addition, transverse beams are present in the middle and end of spans. The bridge bent consists of two square columns with a rectangular cap beam. Column longitudinal reinforcement consisted of 4 $\phi 32 \mathrm{~mm}$ (No. 10) bars on four corners with $\phi 10 \mathrm{~mm}$ (No. 3) stirrups at $305 \mathrm{~mm}$ (12 in) center to center spacing. Furthermore, this typical MSCG bridges built before 1970 have spread or pile foundations. This variation depends on site conditions. And abutments for these typical bridges are generally integral or semi-integral on piles. 


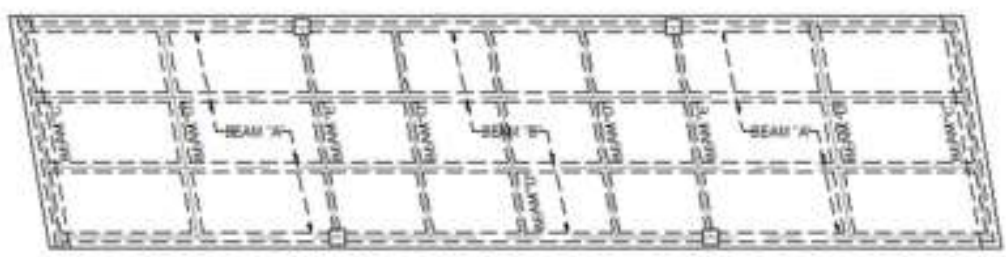

Plan

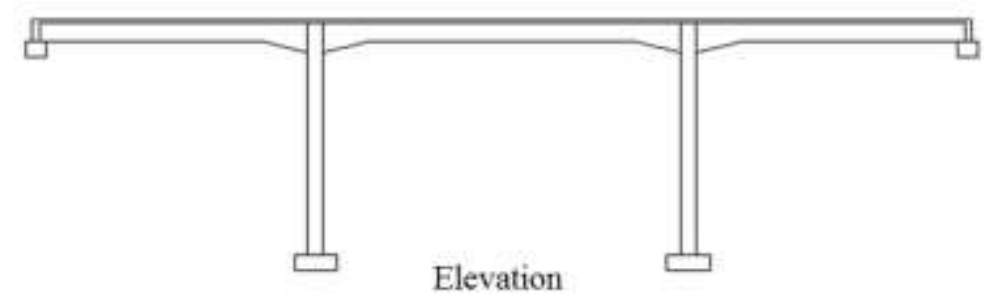

Figure 5-2 Typical Layout of MSCG bridge

Nonlinear static analyses were performed in SAP2000 by using characteristic material properties for reinforcing steel and concrete, and the model described in a study done by Mehary et. al. (2017) also described in Chapter 4.

\subsubsection{Fragility Curve Development Methodology}

Fragility curves are a crucial component of probabilistic seismic risk assessment. Fragility curves define the conditional probability that a predefined damage state is reached or exceeded as a function of an intensity measure (IM). Similar to work done by Shinozuka et al. (2000), the intensity measure considered in this study was the peak ground acceleration (PGA). The strong ground motion time histories were scaled to specific PGAs and grouped in increments on $0.1 \mathrm{~g}$ ranging from $0.05 \mathrm{~g}$ to $0.8 \mathrm{~g}$ (e.g. 0.05 , $0.1,0.2, \ldots, 0.8) \mathrm{g}$. For each group of PGA, the mean and standard deviation of the elastic acceleration response spectra for all the time histories in the group are calculated for the 
considered range of structural period. For each group, three elastic response spectra are developed. (i.e., mean and mean \pm standard deviation). Intersecting the capacity spectrum with the demand spectrum transforms them to acceleration-displacement response spectra (ADRS) format.

Fragility curve is defined as

$$
\mathrm{P}\left[\mu_{\mathrm{d}} \geq \mu \mid \mathrm{PGA}\right]=1-\Phi\left[\frac{1}{\xi} \ln \left(\frac{\mu}{\mathrm{c}}\right)\right] \quad \text { Equation 5-2 }
$$

Where, $\Phi$ - the standard normal distribution function, $\mu_{d}$ - displacement demand ductility, $\mu$ - the displacement demand capacity shown in Table 5-1 and $\xi$ and c are standard deviation and mean values of the corresponding normal distribution

The following equations to obtain the parameters $\xi$ and $c$ given in equations below (Shinozuka M. , Feng, Kim, \& Kim, 2000).

$$
\begin{array}{cc}
\overline{\mu_{\mathrm{d}}}=\mathrm{c} \cdot \exp \left[\frac{\xi^{2}}{2}\right] & \text { Equation 5-4 } \\
\sigma^{2}=\left\{\overline{\mu_{\mathrm{d}}}\right\}^{2}\left[\exp \left(\xi^{2}\right)-1\right] & \text { Equation 5-3 }
\end{array}
$$

The fragility curve generated by Basaez \& Dusicka (2018), for Oregon Specific, multi-bent reinforces concrete bridge bents, the probability of exceedance for the selected damage states is shown in Table 5-2 and Figure 5-3. Even though the seismic demand model is performed for a peak ground acceleration range of $0.05-0.8 \mathrm{~g}$, assuming a lognormal fit for the fragility curves allows extrapolation beyond this range within reason. Comparing the fragility parameters with HAZUS default values, it can be seen that the proposed fragility curve implies a much higher vulnerability than that is defined in 
HAZUS. For a conventionally designed MSCG bridge, the default fragility values presented in HAZUS-MH are given in Table 52 and Figure 55.

For a Multi-span continuous pre-stressed concrete girder, commonly found in the Central and Southeastern United States, Choi Et al. (2003) developed analytical fragility curves. The fragility values and curves are presented in Table 5-4 and Figure 5-5. A comparison between the three fragility curve values is shown in Figure 5-6 for four damage states.

Table 5-2 Fragility curve values for representative as-built MSCG bridge bent (Bazaez \& Dusicka, 2018)

\begin{tabular}{c|c|c}
\hline Damage State & Median & Dispersion \\
\hline \hline Slight & 0.18 & 0.38 \\
Moderate & 0.22 & 0.30 \\
Extensive & 0.27 & 0.31 \\
Collapse & 0.48 & 0.38 \\
\hline
\end{tabular}

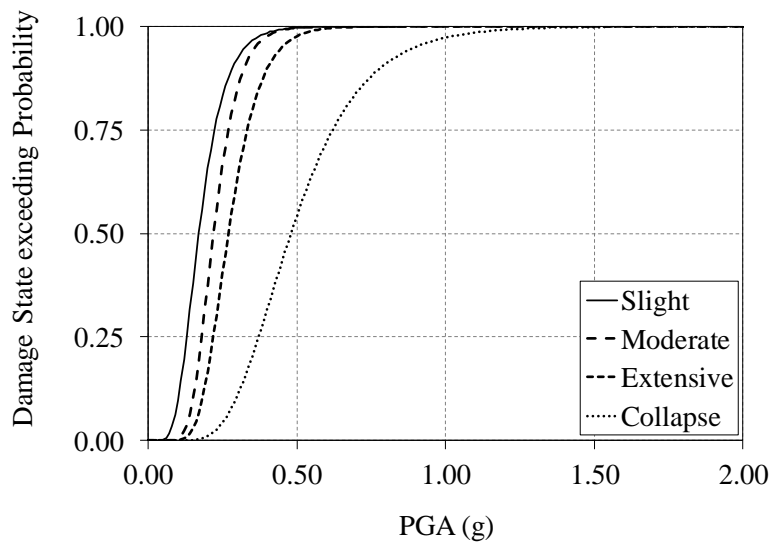

Figure 5-3 Fragility curve values for representative as-built MSCG bridge bent (Bazaez \& Dusicka, 2018) 
Table 5-3 Fragility Curve values for Conventionally Designed 3-Span Bridge (FEMA, 2010)

\begin{tabular}{c|c|c}
\hline Damage State & Median & Dispersion \\
\hline \hline Slight & 0.60 & 0.6 \\
Moderate & 0.90 & 0.6 \\
Extensive & 1.10 & 0.6 \\
Collapse & 1.50 & 0.6 \\
\hline
\end{tabular}

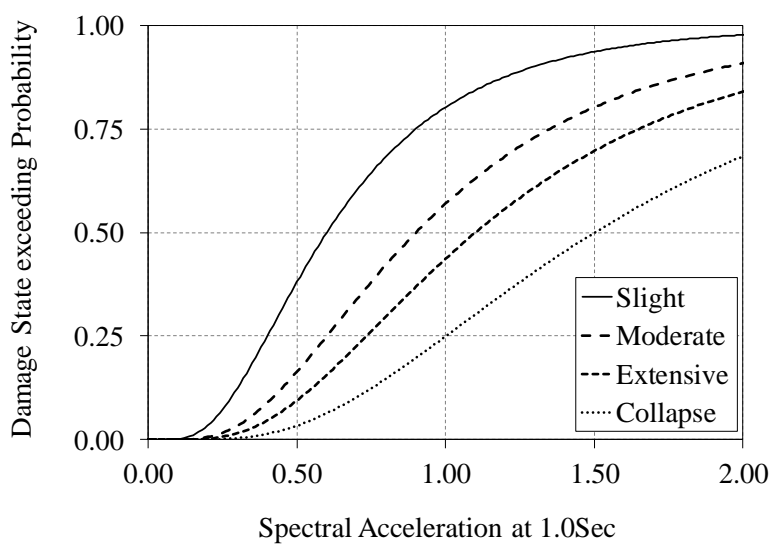

Figure 5-4 Fragility Curves for Conventionally Designed 3-Span Multi-Span Continuous Concrete Bridge (FEMA, 2010)

Table 5-4 Fragility curve values for representative as-built MSCG bridge bent (Choi, DesRoches, \& Nielson, 2003)

\begin{tabular}{c|c|c}
\hline Damage State & Median & Dispersion \\
\hline \hline Slight & 0.16 & 0.70 \\
Moderate & 0.53 & 0.70 \\
Extensive & 0.75 & 0.70 \\
Collapse & 1.01 & 0.70 \\
\hline
\end{tabular}




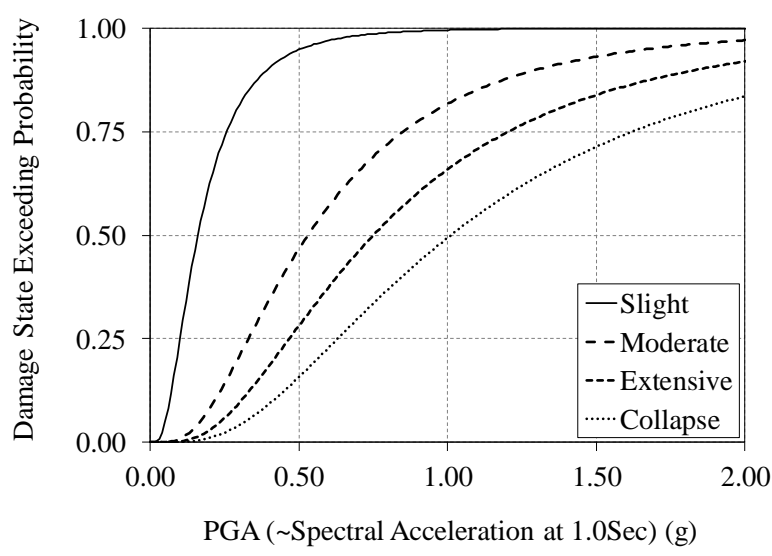

Figure 5-5 Fragility curves for representative as-built MSCG bridge (Choi, DesRoches, \& Nielson, 2003)

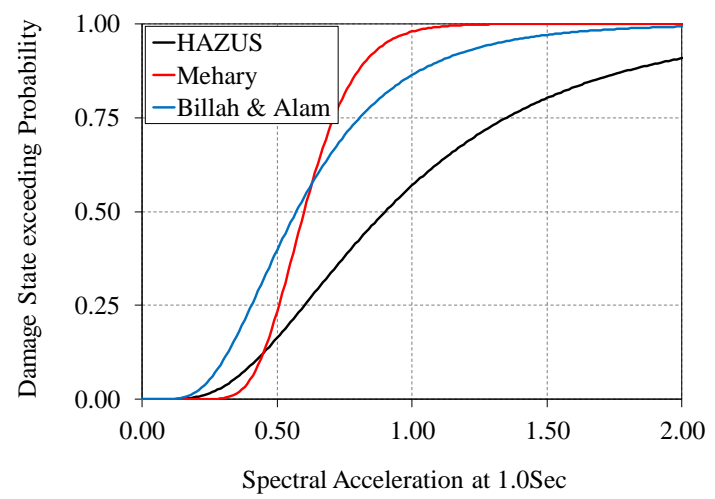

(a)

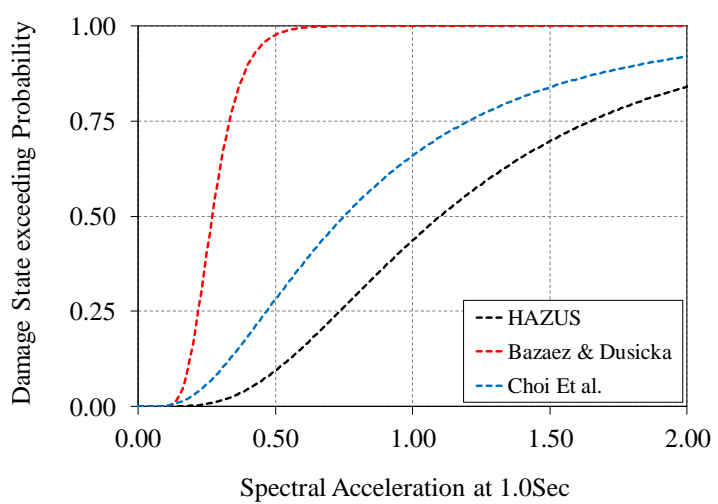

(c)

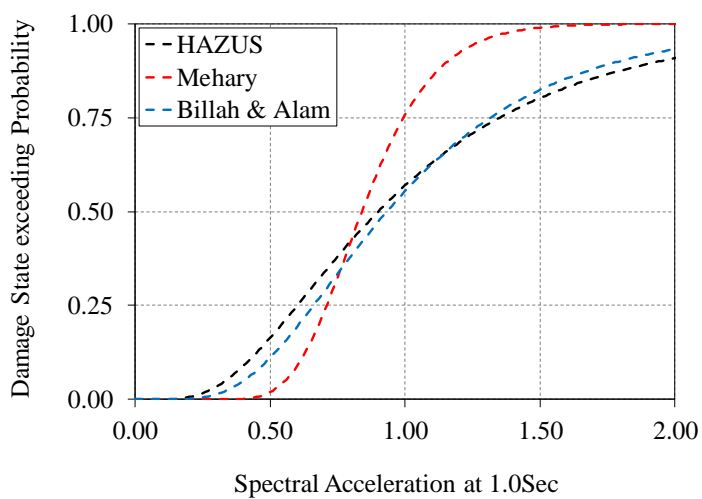

(b)

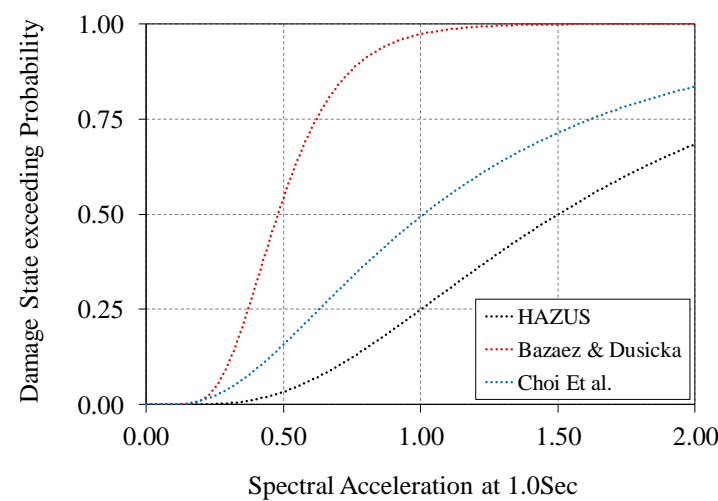

(d)

Figure 5-6 Fragility curve comparison for multi span concrete columns (a) Slight; (b) Moderate; (c)

Extensive; (d) Collapse damage States 
As can be seen from the above figure, the fragility curves developed by Bazaez \& Dusicka (2018) are much lower for damage states moderate, extensive and collapse.

\subsection{Fragility Curve for a Retrofitted Three-Span Concrete Continuous Girder Bridge}

For the CFRP retrofitted bridge, new limit states were defined. Limit state capacities for the retrofitted bridge bent are obtained by transforming the drift limits proposed by Dutta and Mander (1999) to ductility demand of the bridge bent. This limits are presented in Table 5-5. These drift limits proposed for retrofitted RC columns are well documented in literature (Shinozuka et al. (2002) and Kim and Shinozuka (2004)) in seismic fragility assessment of RC columns retrofitted using steel jackets. Furthermore, Roy et al. (2010) experimentally investigated the seismic performance of RC bridge bent retrofitted with CFRP jacket. They also found various limit states values of CFRP retrofitted bridge bent which were similar to that proposed by Dutta and Mander (1999). Therefore, the same limit states were applied in this study.

Table 5-5 Damage/limit state of bridge components (adapted from Dutta and Mander, 1999)

\begin{tabular}{c|c|c}
\hline Damage State & Description & Drift limits \\
\hline \hline No & First yield & 0.005 \\
Slight & Cracking, spalling & 0.007 \\
Moderate & Loss of anchorage & 0.015 \\
Extensive & Incipient column collapse & 0.025 \\
Collapse & Column collapse & 0.050 \\
\hline
\end{tabular}

The yield displacement of the CFRP jacketed column was 0.91 in. (23 mm). Dividing the displacement corresponding the damage states by the yield displacement, 
the ductility demand of the CFRP jacketed bridge column for the four damage states was obtained. Displacement ductility, $\mu$, as the engineering demand parameter (EDP) used to define damage state of the columns. These values the corresponding damage states are given in Table 5-6.

Table 5-6 Damage states for retrofitted bridge

\begin{tabular}{c|c|c|c|c}
\hline \multirow{2}{*}{ EDP } & \multicolumn{4}{|c}{ Damage States } \\
\cline { 2 - 5 } & Slight & Moderate & Extensive & Collapse \\
\hline \hline $\begin{array}{c}\text { Displacement ductility } \\
(\mu)\end{array}$ & $\mu>1.7$ & $\mu>3.7$ & $\mu>6.1$ & $\mu>12.2$ \\
\hline
\end{tabular}

Similar to the methodology employed by Bazaez \& Dusicka (2018), a nonlinear analysis is used to produce seismic fragility of MSCG Bridge retrofitted with CFRP. Two-dimensional (2D) finite-element model scheme modeled in SAP2000 were used in the analytical modeling because of the good agreement between experimental and analytical results for the retrofitted and as-built condition as was discussed in chapter 4 . The CFRP layers are modeled by altering the column fiber models to depict the enhanced confinement as well as the observed slight increase in column stiffness due to the added confinement. In addition, in order to simplify the modelling, the bridge bent is assumed to be supported on rigid foundation.

In this study, the limit states of the retrofitted bridge bent is assumed to follow a lognormal distribution. The uncertainty associated with each median is given in the form of a lognormal standard deviation or dispersion. The values of lognormal standard deviation or dispersion have been obtained following the procedure described in Nielson 
(2005). The probability of exceeding selected damage states is summarized in Table 5-7 and Figure 5-7 for a bridge retrofitted by CFRP.

Table 5-7 Fragility curve values for CFRP retrofitted RC bridge column

\begin{tabular}{c|c|c}
\hline Damage State & Median & Dispersion \\
\hline \hline Slight & 0.53 & 0.50 \\
Moderate & 0.87 & 0.50 \\
Extensive & 1.40 & 0.50 \\
Collapse & 2.12 & 0.50 \\
\hline
\end{tabular}

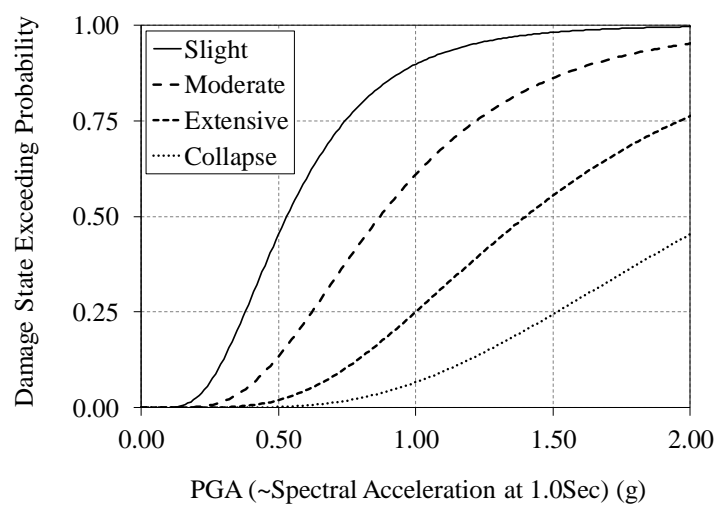

Figure 5-7 Fragility Curves for CFRP Retrofitted MSCG Column

The HAZUS bridge classification considers bridges that are constructed after 1990 as seismically designed. Fragility curve values for a seismically designed MSCG bridge are given in Table 5-8 and the plots of these curves are subsequently given in Figure 5-8. For comparison, fragility curves developed by Billah, Alam, \& Bhuiyan (2013) using carbon fiber-reinforced polymer (CFRP) jacketing of MSCG type bridges are also considered. The resulting fragility curve parameters are presented in Table 56 and the fragility curves are shown in Figure 510. 
Table 5-8 Fragility Curve values for Seismically Designed MSCG Bridge (FEMA, 2010)

\begin{tabular}{c|c|c}
\hline Damage State & Median & Dispersion \\
\hline \hline Slight & 0.90 & 0.6 \\
Moderate & 0.90 & 0.6 \\
Extensive & 1.10 & 0.6 \\
Collapse & 1.50 & 0.6 \\
\hline
\end{tabular}

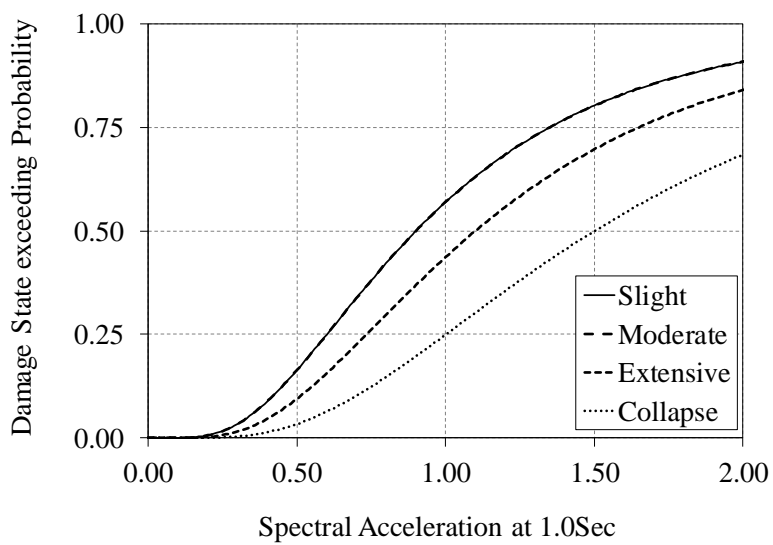

Figure 5-8 Fragility Curves for Seismically Designed MSCG Bridge (FEMA, 2010)

Table 5-9 Fragility curve values for CFRP retrofitted MSCG bridge bent (Billah, Alam, \& Bhuiyan, 2013)

\begin{tabular}{c|c|c}
\hline Damage State & Median & Dispersion \\
\hline \hline Slight & 0.57 & 0.51 \\
Moderate & 0.93 & 0.51 \\
Extensive & 1.55 & 0.51 \\
Collapse & 2.32 & 0.51 \\
\hline
\end{tabular}




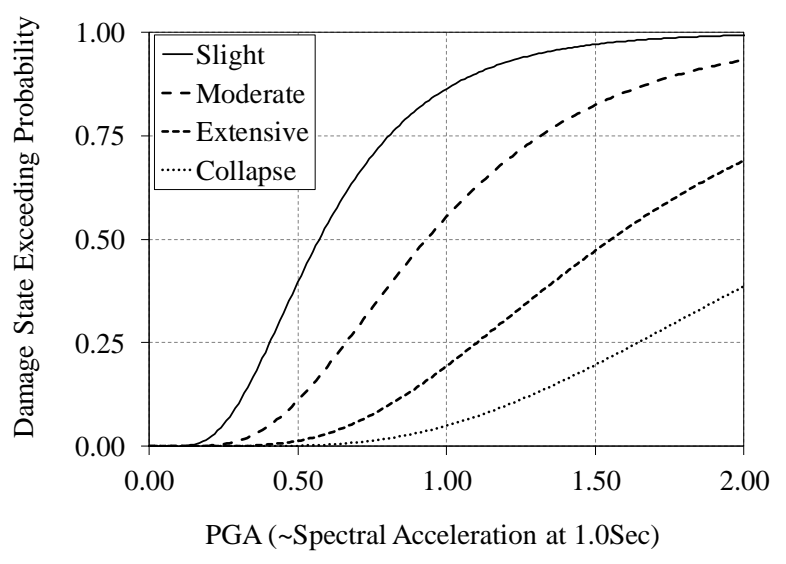

Figure 5-9 Fragility curve for CFRP retrofitted MSCG bridge bent (Billah, Alam, \& Bhuiyan, 2013)

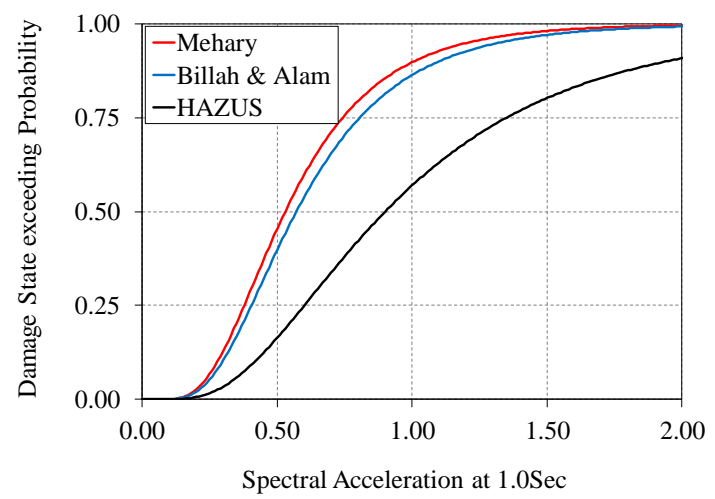

(a)

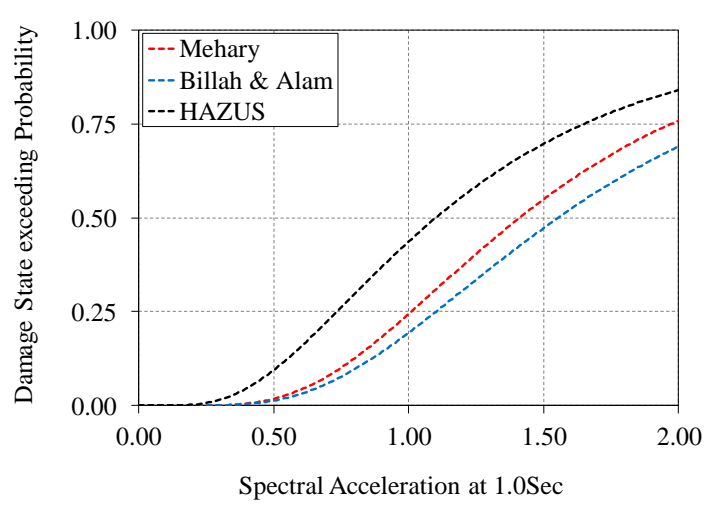

(c)

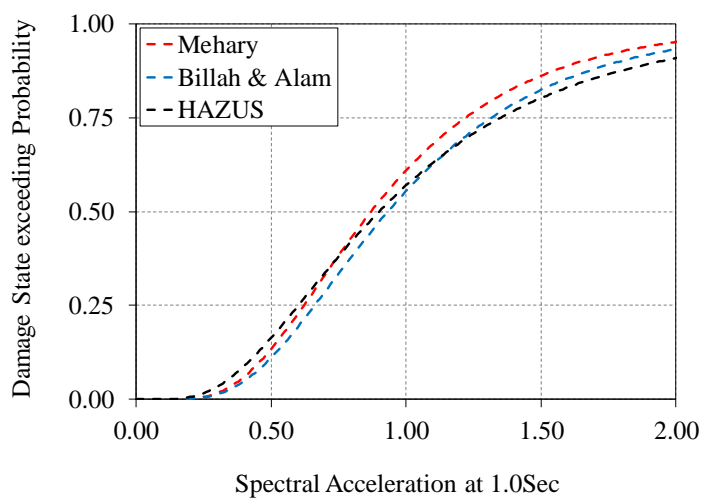

(b)

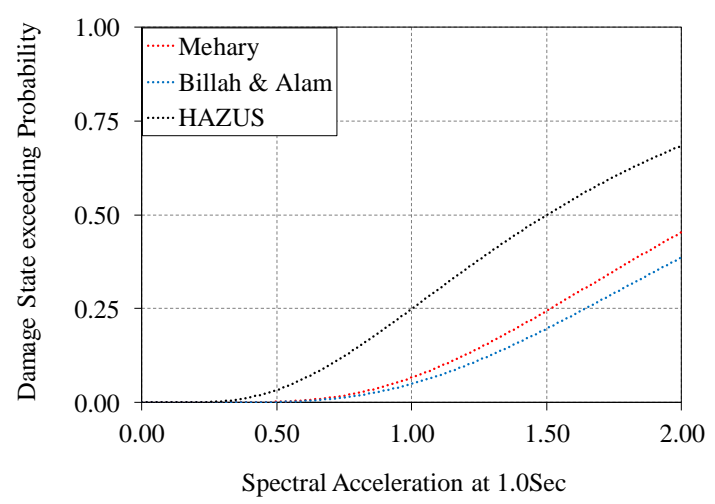

(d)

Figure 5-10 Fragility curve comparison for retrofitted columns (2018) (a) Slight; (b) Moderate; (c)

Extensive; (d) Collapse damage States 


\subsection{Fragility Sensitivity Analysis}

In order to understand the effect of assigned fragility values on the seismic risk assessment outcome, a sensitivity analysis was performed. The effect of increasing the fragility median values to $25 \%$. 50\%, $100 \%$ and $125 \%$ were compared for a Cascadia full length magnitude 9.34 and a Portland Hills magnitude 7 scenario earthquakes. The fragility values for MSCG and MSSC bridges were first considered and results are shown in Figure 5-11 (a) and (b). In both of the analyzed scenarios, as fragility median value increases to 50\%, a sharp increase in the number of bridges that sustain slight damage is seen. This correlates with a decrease in the number of more severe damage observed. However, as the percentage of median value increases above 50\%, all types of damages start to taper down. At $125 \%$ of fragility median value increase a significant drop can be observed in both moderate and severe earthquake damage with an increase in slight damage as compared to current unretrofitted bridges. To evaluate the effect of including all bridges that would benefit from retrofitting, a sensitivity analysis was conducted on the effect of increasing fragility median values for all bridges constructed after 1990. The sensitivity analysis was done by changing the fragility median values for all bridges that were built before 1990 Figure 5-11 (c and d). In this scenario, the trend is similar but is even more pronounced in the reduction of all types of damages as well as a significant lower cost for repair and replacement. This can be explained by the observation made in the vulnerability assessment that next to MSCG and MSSC type bridges, simply supported concrete and steel bridge types sustain damages ranging from slight to collapse. Hence, retrofit designs that would increase the ductility levels of these bridge types to a desired level could take in to consideration the results of the sensitivity study. 
The CFRP retrofit recommended in this study will increase the median fragility of the bridge by about $140 \%$. However, a retrofit measure that would increases the fragility by $50 \%$ to $75 \%$ would significantly reduce the amount of damage and associated cost.

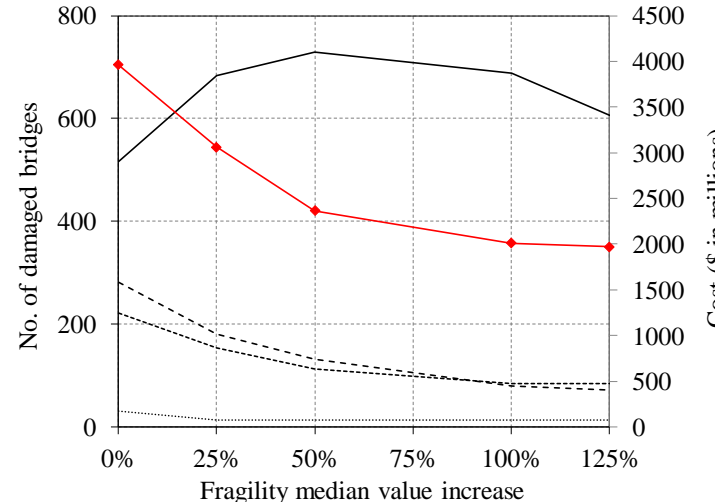

(a)

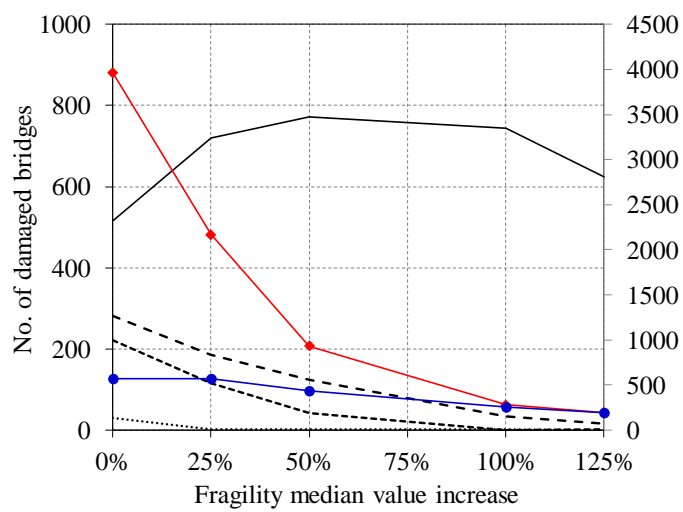

(c)

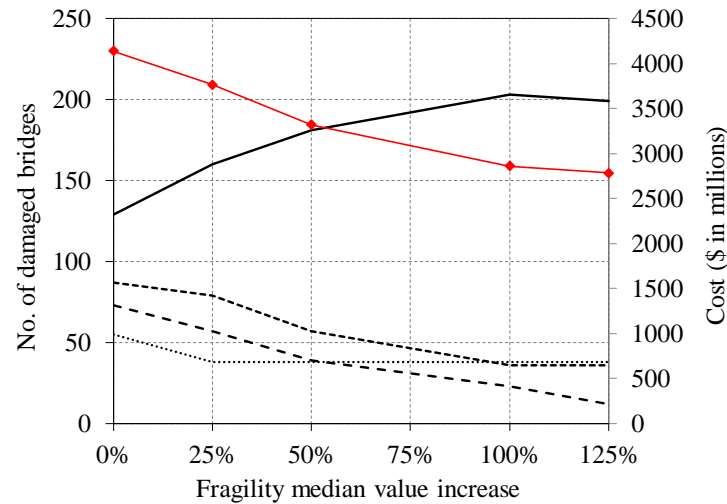

(b)

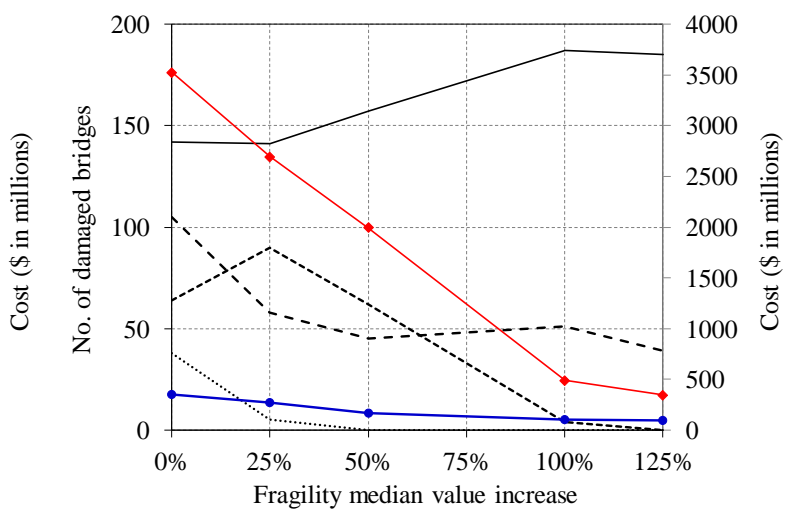

(d)

\begin{tabular}{llll|}
$\longrightarrow$ & Slight & -- & Moderate \\
--- & Extensive & $\cdots \cdots$ & Collapse \\
$\multimap$ & Repair/Replacement Cost & $\rightarrow$ & Travel Time Delay Cost/Day \\
\hline
\end{tabular}

Figure 5-11 Sensitivity Analysis, (a) CSZ M9.34 Scenario (MSCG and MSSC), (b) Portland Hills M7 (MSCG and MSSC), Scenario, (c) CSZ9 M9.34 Scenario (all bridges built <1990), and (d) Portland Hills M7 Scenario (all bridges built <1990) 


\section{CHAPTER 6}

\section{STATE OF THE ART ON PRIORITIZATION OF BRIDGES FOR SEISMIC RETROFITTING}

\subsection{General}

Many screening and prioritization methods have been proposed in the past. Most of these methods develop a seismic rating system first, and then use the results of the rating exercise to prioritize the inventory. Other methods compare the severity of expected damage for each bridge in the inventory for the same earthquake, and rate bridges accordingly. Recent advancement in seismic risk assessment methods has led to the development of fragility functions for specific classes of bridges. These in turn have led to loss estimation methodologies for highway systems and estimation of the effect on system performance as measured by traffic flow (Werner S. D., et al., 2006).

These three major methods have been mentioned in the Seismic Retrofitting Manual for Highway Structures (Buckle, et al., 2006). The methods are Indices Method, Expected Damage Method and Seismic Risk Assessment Method. A brief overview of the literature on the above mentioned methods and other approaches is provided below.

\subsection{Indices Method}

Indices method is the simplest of the three methods. In this method indices are used to characterize the bridges' vulnerability and hazard level and are then combined to give a single rating for each bridge. Indices range from 0 to 10 and are based on conservative, semi-empirical rules. Prioritization is determined by this rating together 
with a qualitative evaluation of importance, redundancy, non-seismic issues, and socioeconomic factors.

California Department of Transportation (Caltrans) (Roberts, 1991), Applied technology Council (ATC-6-2, 1983), The Illinois Department of Transportation (IDOT) (Woodward-Clyde Consultants, 1991), Washington State Department of Transportation (WSDOT) (Babaei \& Hawkins, 1993) etc. have used Indices Method to characterize the structure vulnerability and hazard level and then combining to give a single rating for each bridge. In all these cases the three major variables considered were the vulnerability of the bridge, seismicity of the bridge site and the importance of the bridge.

\subsection{Expected Damage Method}

In the Expected Damage Method, the level of expected damage for each bridge in the inventory is compared for the same earthquake. Severity of damage is measured either by sustained damage states or by estimating direct economic losses. The higher the expected damage and/or loss, the higher the priority for retrofitting. Fragility functions

are used to address the uncertainties in ground motion and the unpredictability in soil and structure properties and to estimate damage-state probabilities (Buckle, et al., 2006). Furthermore, a qualitative assessment of indirect losses, network redundancy, and nonseismic issues are included in the final ranking assessment.

Cherng, et al. (1992) presented a method that considered retrofit priority and amount of upgrading. They used a retrofit criterion instead of the concept of priority index. The retrofit criterion considered the consequence of failure of the component that included cost of reconstruction, casualty and loss of functionality; loss due to network 
failure; and retrofit cost for a component increased from before-retrofit strength coefficient to after-retrofit strength coefficient. The main objective of this method was to maximize the net profit benefit for a given budget putting into consideration the network reliability and the criticality of the bridge to the community and the bridges' vulnerability to seismic hazard.

Kim, et al. (1992) proposed a GIS-based regional risk analysis program with a purpose to interactively study the vulnerability of bridges in a regional highway system. Basöz and Kiremidjian (1995) on the other hand proposed a prioritization/ranking method that was more comprehensive and was based on vulnerability and importance. In this approach, vulnerability and seismicity were interrelated and their relationship was considered in the ranking process. Vulnerability was assessed by first classifying bridges according to the material type, structure type and other factors such as number of spans, span continuity, column bents, abutment type, etc. The bridge location and soil condition were used to compute a seismic hazard curve. Corresponding ground-motion damage relationships for the bridge class were then used to evaluate the vulnerability parameter. The consequence of failure of a bridge to public safety and the socio-economic benefit of a community was reflected by the Importance Factor. Transportation lifeline network analysis and decision analysis were the main tools used to evaluate the importance criterion. The Importance Factor was evaluated as a function of public safety, emergency response, long term economic impacts, defense route, interaction with other lifelines and historical significance of the bridge. Assessment of importance depended on the decision makers values. The bridges were then ranked as a function of their vulnerability and importance. 


\subsection{Seismic Risk Assessment Method}

Seismic Risk Assessment Method is the most complex but least conservative of all three methods. In this approach analysis of the highway network is performed for a given seismic hazard level and the resulting damage states of the bridges are used to estimate the effect on highway system performance. A number of prioritization options can be considered, and the impact on post-earthquake system performance is considered in establishing prioritization criteria. This is achieved by using the methodology to assess how much the seismic performance of the overall roadway system performance is improved when different sequences of bridge retrofits are followed. This performance is evaluated in terms of losses due to travel time delays. (Werner S. D., et al., 2006). Independent qualitative assessment of non-seismic issues and socioeconomic factors are also included in the final prioritization criteria. In the Seismic Risk Assessment Method, systemic effects associated with the loss of a given bridge and the combined effects associated with the loss of other bridges in the highway system are taken into account. This consideration of both systemic and combined effects is what can provide a much more rational basis for establishing seismic retrofit priorities.

Basöz and Kiremidjian (1995) have included network analysis when assessing importance criterion. However, ranking of bridges depends on the bridges function and criticality in the network system as an entity. The systemic effects associated with the loss of a bridge and the combined effects associated with the loss of other bridges in the highway system are not considered. Shinozuka et. al. (2008) developed a retrofit 
prioritization strategy which maximizes the expected benefit from seismic retrofitting under any future earthquake.

Werner, et al. (2004) described a methodology for probabilistic or deterministic seismic risk analysis (SRA) of highway systems. The process was programmed into a public-domain software package named REDARSTM 2 (Risks from Earthquake DAmage to Roadway Systems). Werner, et al (2006) illustrated REDARS2 capabilities and applications. REDARS2 addresses system characteristics that affect post-earthquake traffic flows such as the highway system network configuration; locations, redundancies, and traffic capacities and volumes of the system's roadway links; and component locations within these links. The overall project was carried out by the Multidisciplinary Center for Earthquake Engineering Research (MCEER) under the sponsorship of the Federal Highway Administration (FHWA). Werner, et al. demonstrated how REDARS2 can be used when establishing priorities for retrofit of bridges and other roadway components. REDARS2 enables users to consider how various prioritization options could impact post-earthquake system performance. Another application of REDARS2 is as a guide in the planning of the most effective post-earthquake response and recovery strategies in the presence of damage to the highway system. The REDARS2 output can be employed in prioritization options that would optimize the effectiveness of PostEarthquake Response and Recovery operations after an earthquake.

ODOT (2009), in an effort to better utilize any future funding for seismic retrofit, came up with a preliminary prioritization strategy. The methodology attempted to capture the major factors that would make the prioritization process reasonable and 
understandable. What made this method different from the other methods mentioned above was, instead of prioritizing bridges as single entities, the method looked at the major highway routes. The key criteria in prioritizing the system were improving longer stretches of highways with lower costs at the same time considering areas that were most populated. Hence route length and the average daily traffic were assumed to be very important factors. Retrofit cost was also compared to the maximum earthquake loss for the same highway segment. The route that produced the largest reduction in cost after retrofit was given the highest priority.

\subsection{Other Methods}

Transit New Zealand (1998) developed a twelve stage screening process for evaluating bridges within New Zealand with regard to seismic hazards. The process used estimates of the bridge's vulnerability, its probability of experiencing high magnitude earthquakes, and the impact to the economy if the bridge is damaged and out of service. The economic ranking indicator took into consideration the relative consequences and probabilities of loss of serviceability of a bridge. It was calculated as a ratio of the product of probability factor of PGA value, service life factor and traffic cost parameter to the rough cost of retrofit. The traffic cost parameter was calculated as a factor of number of days to reinstate the bridge to existing traffic capacity, number of days for which the detour would have to be used until the crossing can be reopened with either repaired or temporary crossing, annual average daily traffic, length of normal and detour route, and journey speed along normal and detour route. Risk events were then ranked taking into account the consequence and relevance of the indicators relating to safety of 
people, ratio of retrofit to depreciated value of bridge, ratio of retrofit to replacement, and economic ranking order. This list was then used to rank bridges considered to warrant detailed seismic assessment. Seville and Metcalfe (2005) used a GIS framework to combine the seismic hazard map showing the peak ground acceleration (PGA) for given return periods and the probability of exceeding certain demand intensities in the road network system. They integrated the vulnerability analysis of bridges using the outputs of the screening process for bridges performed by Transit New Zealand (1998). They included liquefaction hazard maps in the model. They then assessed other risks that may follow an earthquake such as landslides, tsunamis and rock falls by consulting experienced geologists. They finally estimated the total risk for each section of the state highway network that could be affected by seismic events.

Wang (2006) presented a generic decision support system for selecting a costeffective bridge seismic retrofit strategy implementing analytical fragility curves. An Economic Index (EI) was proposed to identify the most cost-effective solution when decision-makers face multiple alternatives. Bridge fragility curves corresponding to various cost levels of retrofit were constructed to compare their effectiveness. The approach provided engineers and owners with a quantifiable solution for selecting retrofit alternatives. The four levels of retrofit were classified as do-nothing, basic level retrofit, full-blown rehabilitation and total replacement. To calculate the EI, first typical bridge fragility curves before and after various level of retrofit were generated and plotted on an acceleration coefficient vs. relative displacement ductility ratio graph. Then the area between the curves and the cost increment between the different retrofit measures was determined. To evaluate the EI, a cost-benefit ratio was computed as the ratio of the area 
between curves and the incremental cost. The highest value of EI means it was the most economical solution.

Padgett (2010) considered lifetime sustainable performance of systems applied to bridge retrofit and prioritization in the face of seismic hazards. Padgett took a scientific approach to mitigate the risks to bridges posed by earthquakes, while balancing broader goals for sustainability. Life-cycle costs and cost-benefit analysis were elaborated as a key metrics for selecting seismic retrofit of bridges in Central and Southern United States. As an extension to that, Padgett considered seismic upgrade of bridges prioritized based on multiple sustainable impact metrics including social and environmental consequences of seismic damage. Padgett did a case study life cycle cost and cost benefit analysis of seismic retrofit in a multi-span continuous concrete girder bridge with nonseismic detailing that is common of the Central and Southern US. In this study the expected life-cycle costs were evaluated for the bridge in its as-built and retrofitted condition. The benefit of a retrofit was evaluated as the difference between the expected present life cycle cost of the bridge without retrofit and present life cycle cost of the bridge with retrofit. A cost-benefit ratio (CBR) was calculated as the ratio of net present benefit of the investment in retrofit to the initial cost of the retrofit. CBR greater than one meant a positive return on savings. A retrofit option with the largest CBR had a larger expected saving in losses over remaining life per amount of money invested in mitigation.

Stevanovic and Nadimpalli (2010) prepared a study on seismic vulnerability and emergency response analysis of Utah DOT lifelines. They estimated the delay-based user 
costs following an earthquake and also helped UDOT make informed decisions on disaster mitigation plans. Stevanovic and Nadimpalli selected two scenario seismic events for the study. Since it was impractical to attempt to improve all vulnerable bridges for seismic hazards, they concentrated on selecting road segments (links) for rehabilitation that were vulnerable in one scenario but could still carry a considerable amount of detour traffic in the other scenario. Links that were damaged under both earthquake scenarios were classified as most vulnerable links. Links with the highest increase in traffic under both earthquake scenarios were classified as most critical links. Finally, a list of links that were both critical and vulnerable was prepared for both scenarios. These lists can be useful to effectively utilize the rehabilitation resources and to reduce the vulnerability of the critical link.

\subsection{Summary}

The indices method is the easiest and most conservative of the three methods. In this method seismic rating systems are developed first and are used to prioritize the inventory. The expected damage method on the other hand compares the severity of expected damage for each bridge in the inventory for the same earthquake, and bridges are rated accordingly. Recent advancement in seismic risk assessment methods has led to the development of fragility functions for specific classes of bridges. These fragility functions address the uncertainties in ground motion and the unpredictability in soil and structure properties and can be used to estimate damage-state probabilities. The Seismic Risk Assessment Method is the most advanced of the three methods. The methodology 
makes it possible to estimate loss of highway systems and the effect on system performance as measured by traffic flow.

In both indices method and expected damage method each bridge is treated as an individual entity only and without regard to how the extent of its damage from earthquakes may impact highway system performance. Both methods represent the importance of the bridge as a traffic-carrying entity only and use average daily traffic, route type and detour length as parameters in prioritization. These criteria do not account for the systemic effects associated with the loss of a given bridge or the combined effects associated with the loss of other bridges in the highway system. The Seismic Risk Assessment method is the most complex and least conservative of all three. However, detailed structural and geotechnical information is required.

The research in this study adapts the approach that focused on improving longer segments of highways with the available funding. Similar to the approach taken by ODOT (2009), and Stevanovic and Nadimpalli (2010), this prioritization methodology will also focus on assessing the vulnerability of a highway segments under seismic loading. Different routes have different retrofit needs and have different seismic risk exposure. The desired outcomes are for consideration and prioritization of highway segments or routes instead of the conventional approach of prioritizing individual bridges. This approach will address how much the seismic performance of the overall roadway system is improved when different sequences of route or segment retrofits are followed. 


\section{CHAPTER 7}

\section{PRIORITIZATION APPLICATION TO OREGON'S HIGHWAY TRANSPORTATION NETWORK}

\subsection{General}

With a majority of state owned bridges designed and built between 1950 and 1980, the state of Oregon would face a devastating post-earthquake situation if a major seismic event occurred in the state. Currently, there is not enough funding available for ODOT to retrofit all state bridges with seismic deficiencies. The purpose of this study is then to construct a methodology to prioritize bridges for retrofit in the State of Oregon.

This study develops the methodology for seismic retrofit prioritization of highway segments based on benefit-to-cost ratio. The basic procedure is as follows seismic risk assessment algorithm developed by Basoz and Mander (1999). The algorithm considers the damage states of bridges defined by fragility curves for a given ground shaking event. Four damage states applicable to highway bridges and consistent with those defined in HAZUS (FEMA, 2010) were used in this study, namely, slight, moderate, extensive, and complete. Bridges are first located (longitude and latitude) and classified based on their structural characteristics. The classification follows the 28 classifications that are described in HAZUS (FEMA, 2010). Then the peak ground acceleration (PGA), spectral accelerations ( $\mathrm{Sa}[0.3 \mathrm{sec}]$ and $\mathrm{Sa}[1.0 \mathrm{sec}])$ for each bridge are evaluated by means of a GIS software. The ground shaking damage functions for each bridge type, described by a fragility curve are then compared with the ground accelerations and the ground shaking related damage state probabilities were evaluated. 
A statewide seismic hazard assessment model had been developed and has been used to conduct seismic vulnerability analysis, which was widely distributed and publicized (ODOT, 2009). Dusicka et al. (2007) and Mehary \& Dusicka (2012) developed a GIS model of the roadway and bridge network for the State of Oregon using technology developed for the Federal Highway Administration called REDARS2. The statewide hazard assessment model developed for ODOT was also executed using the said software. REDARS2 and HAZUS-MH use the same Damage Functions for Bridges. REDARS2 uses HAZUS99-SR2 model for estimating damage state of bridges which makes use of damage functions developed by Basoz and Mander (1999). The results have been useful in analyzing the vulnerability of the highway system and have highlighted the need for prioritization method.

Oregon Department of Transportation (ODOT) recognizes that it is impossible to retrofit all vulnerable bridges in the near future. Instead, a retrofit strategy needs to be developed to prioritize and enumerate the retrofit costs of routes or segment that would result in the most benefit. ODOT had developed a cost effective strategy to select bridges that would undergo seismic retrofit. In the previous chapters, bridge seismic retrofit practices in Oregon were reviewed along with the current state of the art in bridge fragility curve development with the intention to establish a framework for further investigation of the development of analytical fragility curves for bridges in their retrofitted conditions.

Retrofit measures that is considered is CFRP wrap of the column. The benefit of a retrofit will be evaluated by comparing the expected present value of the losses without 
retrofit and present value of the losses with retrofit. A highway route that is most critical will give the highest return on investment by reducing system-wide traffic disruptions and economic losses, as well as overall repair costs. Cost-Benefit Analysis of highway segments will be assessed and the highway segments will be ranked according to largest expected savings in losses over the remaining life per amount of money invested in retrofitting.

\subsection{Cost-Benefit Analysis for Prioritization}

Economic and social costs and benefits are the key matrices used in the costbenefit analysis for seismic retrofit prioritization. In this study the expected costs and losses were evaluated for the bridge in its as-built and retrofitted condition. The benefit of a specific retrofit was evaluated as the difference between the expected costs of the bridge without retrofit and the bridge with retrofit. Costs without retrofit included repair and replacement of the bridge, travel time delays and cost associated with causalities. Costs with retrofit included damage to the bridge and the cost of the retrofit. Similar to the study by Padgett (2010), the Cost-Benefit ratio was then calculated as the ratio of net present benefit of the investment in retrofit to the initial cost of the retrofit. A retrofit option with the largest benefit-to-cost ratio has a larger expected return per amount of money invested in retrofitting. The schematic in Figure 7-1 shows the procedures in performing the cost-benefit analysis. 


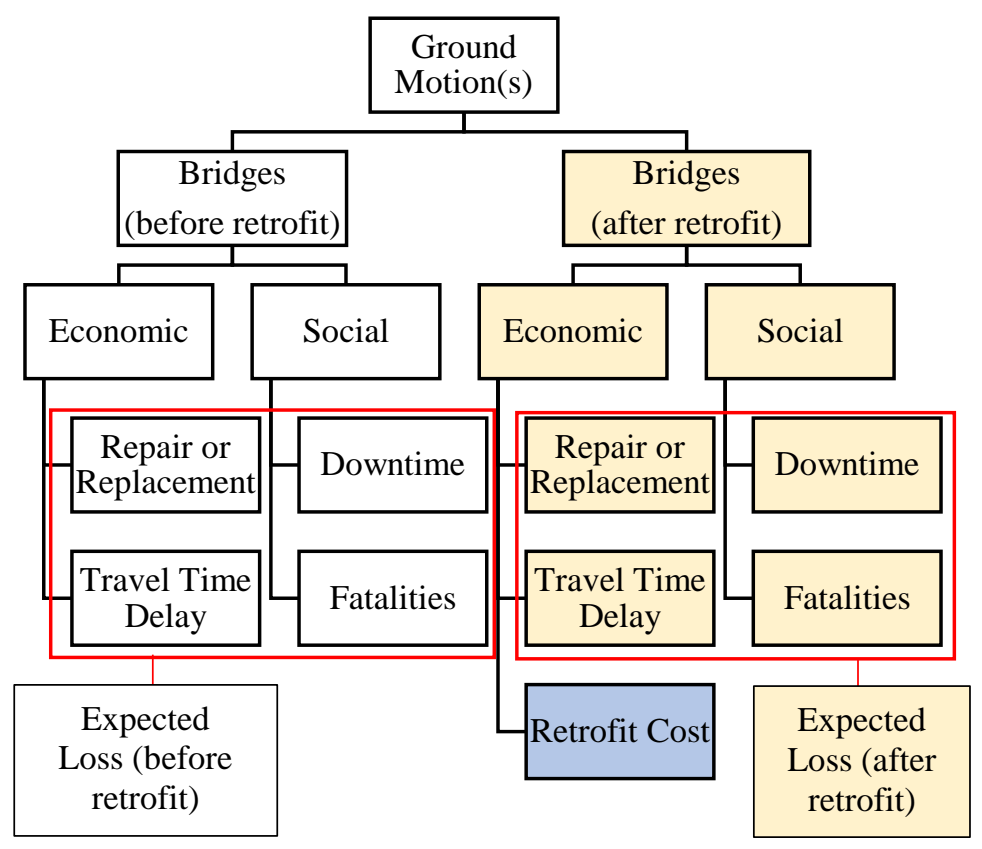

Figure 7-1 Schemathic of Cost-benefit Analysis for Prioritization

\subsection{Economic Module}

\subsubsection{Expected Loss}

The economic module comprises the value of investment on the highway bridges in the form of retrofits and expected losses after sustaining a seismic event. These expected losses include loss due to replacement cost, repair cost and travel time losses. And the benefit is defined as the difference between values of losses without retrofit (Expected Loss before retrofit) and the present value of losses after retrofit (Expected Loss retrofitted) as shown in Equation 7-1.

$$
\text { Benefit }=\text { Expected Loss }_{\text {before }} \text { retrofit }- \text { Expected Loss } \text { retrofitted }
$$

Equation 7-1 


\subsubsection{Cost-Benefit Ratio}

Benefit to Cost Ratio (BCR) a measure of return on investment. BCR is used to compare the efficiency of a number of different investments. It is the ratio net profit to investment (Equation 7-2). In this case the net profit is the 'benefit' and the initial cost of retrofit is the investment. A BCR of less than one indicates a negative return on investment (loss). Hence the retrofit with the largest BCR has the most expected savings.

$$
\mathrm{BCR}=\frac{\text { Benefit }}{\text { Retrofit Cost }} \quad \text { Equation 7-2 }
$$

\subsection{Social Module}

\subsubsection{Downtime}

Downtime, which is the amount of time it takes to repair and restore a highway bridge after earthquake damage is given by restoration curves that were developed based on a best fit to ATC-13 data for the four damage states. However, these assumptions come with some uncertainties in estimating downtime such as the availability of resources to make the repair or replacement, accessibility to component, environmental and regional regulations. Single bridges on some major routes may be replaced with in a year. However, it will probably take over 5 years to replace $70+$ bridges due to limited resources. Completely damaged links contribute more to the total delay costs. This is not only due to their severity of damage, but also due to larger rehabilitation periods required. Another issue in recovery of the network system is that some streets cannot carry the increased traffic volumes that could possibly be diverted to them. Availability of resources to make the repair or replacement, accessibility to components, environmental 
and regional regulations should be studied and applied in the estimation of downtime of a bridge component to make estimates of downtime. Therefore, the downtime estimates were noted but were not used in the prioritization methodology.

\subsubsection{Fatalities}

The ability to estimate casualties from a seismic hazard is a great tool in the costbenefit analysis. The census tract data for Oregon has been gathered from U.S. Census Bureau and is used in the analysis. The causality estimate for the retrofitted state and unretrofitted state for one lifeline route at a time was done and comparisons were made to aid in the cost-benefit estimation to aid in prioritization for seismic retrofit of the selected lifeline highway segments. A VSL value of $\$ 9.6$ million is considered as the cost of reducing the number of deaths by one.

\subsection{Cost-Benefit Assessment Results}

This section demonstrates the application of cost-benefit analysis for prioritization of highway segments for seismic retrofit.

\subsubsection{Assessing Benefit of Retrofit}

In this deterministic seismic risk analysis, USGS generated ShakeMaps were selected and earthquake ground motions were estimated throughout the transportation system. Once the ground motion hazards were estimated, direct and indirect system losses were evaluated. Transportation network analysis then gave an estimate in systemwide travel time delays. The economic module in then estimated losses due to travel time delays. 
The analysis results presented in Chapter 3 started with an assumption that the default published fragilities by Mander and Basoz (1999). These results were used as baseline values. In this section, the previously selected fragility curves that are expected to better represent multi-span continuous concrete girder bridges were utilized. The analysis was done by changing the fragility curves values of multi-span continuous concrete MSCG girder bridges in the transportation network one highway segment at a time, for the highway segments that were selected by ODOT as top priority for rescue and recovery.

The fragility curves of MSCG bridges were updated with fragility curve values developed by Choi Et al (2003) and the fragility curves developed in this study for asbuilt case and CFRP retrofitted case, respectively. Figure 7-2 compares fragility curves for the two cases.

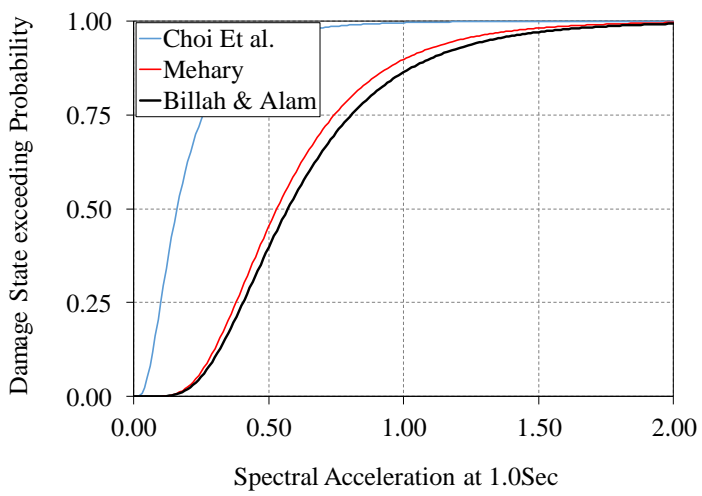

(a)

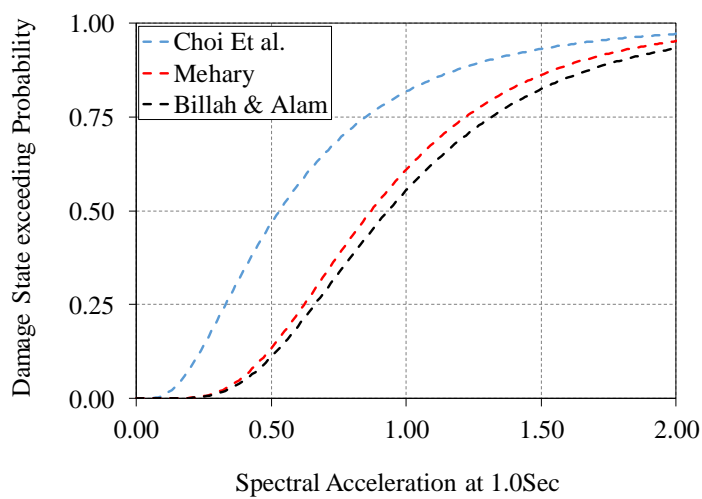

(b) 


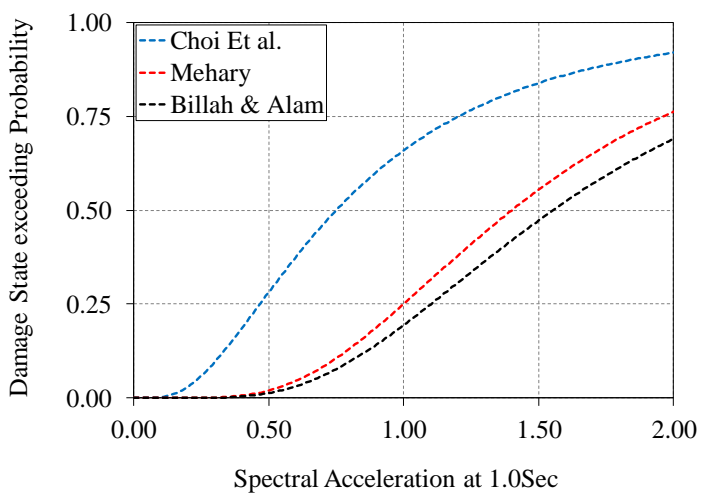

(c)

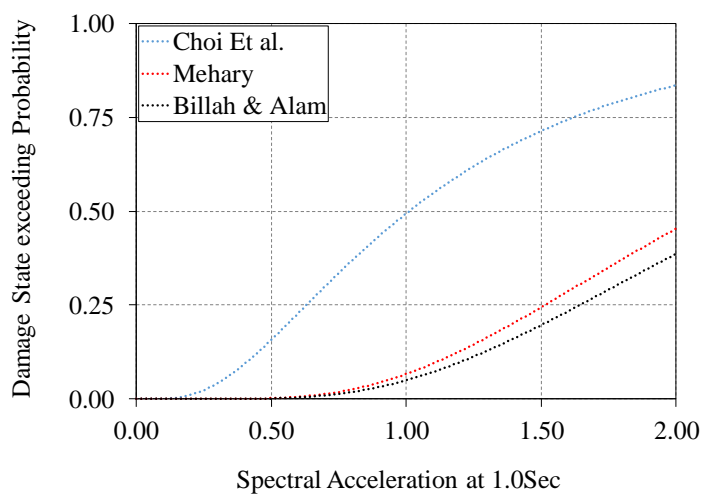

(d)

Figure 7-2 Fragility curve comparison for as-built and retrofitted columns (a) Slight; (b) Moderate; (c) Extensive; (d) Collapse damage States

The cost benefit assessment of retrofitting was studied by first changing the fragility curves for all MSCG bridges to a retrofitted values developed. MSCG bridges in the network. MSCG bridges make up 38\% of the bridges in the study. In the analysis, the number of MSCG bridges damaged ranged from 6 to over 620 for the different earthquake scenarios considered. The damages range from slight to extensive with no collapse. However, when looking at the rest of the bridges in the network, it was observed that most of the bridges that collapsed are the multi column simply supported bridge types. Therefore, since the fragility curves for CFRP retrofitted bridge bents developed by Billah Et al (2013) were for multi column bent concrete bridges, the same values were adopted as the fragility values for a retrofitted multi span simply supported concrete (MSSC) bridges. The results of the analysis are shown in $\quad$ Table 7-1, Table $7-2$ and

Table 7-3. From Table $7-1$, it can be seen that on average, there is a $60 \%$ reduction in the number of damaged bridges for the case where all MSCG bridges were retrofitted and close to $70 \%$ reduction when both MSCG and MSSC bridges were retrofitted. Table 7-2 and

Table 7-3 also show the corresponding cost for the unretrofitted and retrofitted cases for all the earthquake scenarios. Even though, as much as $90 \%$ of MSCG bridges were damaged during the analysis, the estimated damages sustained the bridges 
were mostly slight damage states. Hence, it was obvious that retrofitting the MSCG bridges alone will not be as cost effective. However, when both MSCG and MSSC type bridges were retrofitted by changing fragility values for both bridge types, it can be seen that the benefit outweigh the cost for the more demanding Cascadia and Portland Hills scenarios. Therefore, for the prioritization exercise, both MSCG and MSSC bridges will be retrofitted one lifeline highway segment at a time and results will be compared to make the prioritization ranking. 


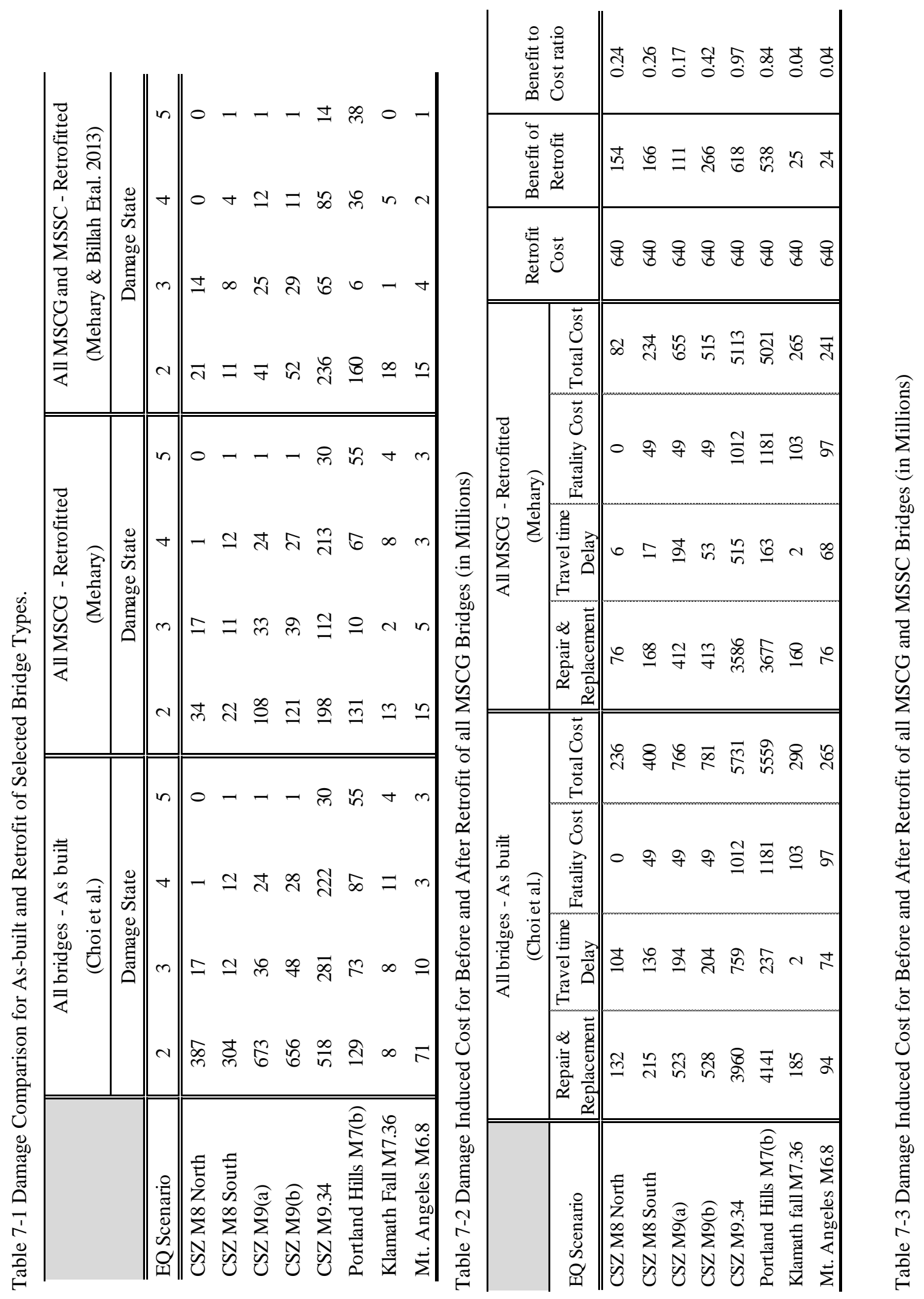




\begin{tabular}{|c|c|c|}
\hline 垔: & & 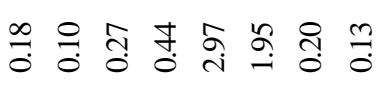 \\
\hline 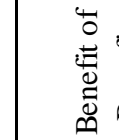 & & 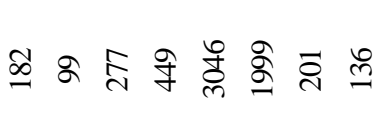 \\
\hline 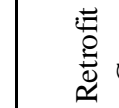 & & 志志志志志志志苞 \\
\hline & 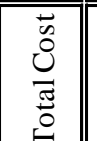 & 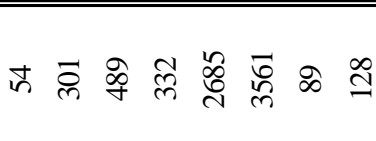 \\
\hline 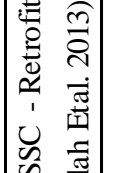 & 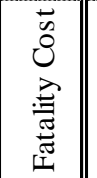 & ○ g g g 常只。 \\
\hline 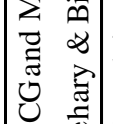 & 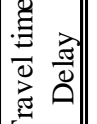 & 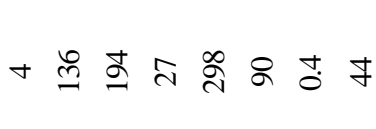 \\
\hline ₹ & 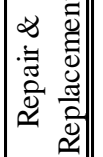 & 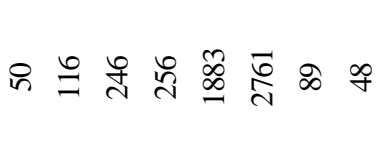 \\
\hline & 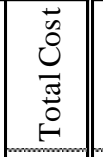 & 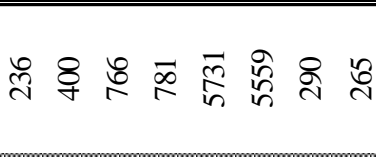 \\
\hline 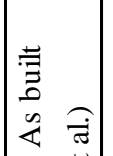 & 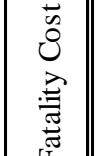 & $\circ g \circ g \stackrel{\simeq}{\varrho} \stackrel{\infty}{\Rightarrow} \cong$ \\
\hline 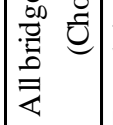 & $\mid$ & 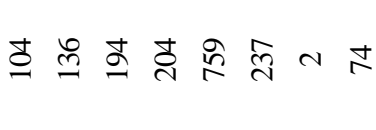 \\
\hline 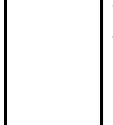 & 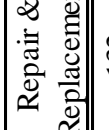 & 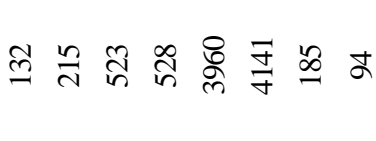 \\
\hline & 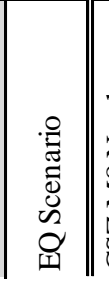 & 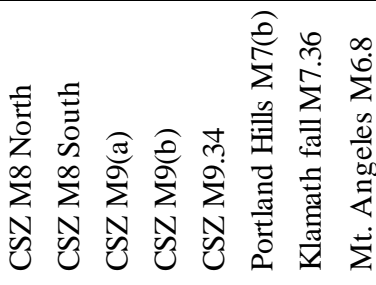 \\
\hline
\end{tabular}




\subsubsection{Retrofit Prioritization}

The prioritization methodology in this study focuses on assessing the vulnerability of a highway segments under seismic loading and adapts an approach with the goal of improving longer segments of highways with the available funding. This approach addresses how much the seismic performance of the overall roadway system is improved when different sequences of route or segment retrofits are followed. Twelve segments were selected based on their importance to the overall highway network system. The effect of retrofitting each segment is quantified based on an estimated direct and indirect cost due to damaged bridges. Economic and social costs were computed and compared. For the retrofit, the top two bridges types in Oregon's bridge inventory, MSCG and MSSC bridges were selected. The prioritization methodology started by analyzing all the bridges in the study are in their current state. Following, all the available MSCG and MSSC bridges were first retrofitted by changing the fragility values. The two assessments serve as a baseline for the next steps.

Then each route retrofits were followed, again by retrofitting all the available MSCG and MSSC in the MSCG and MSSC in the segment under consideration. Six different seismic hazard scenarios were applied scenarios were applied - three Cascadia subduction events and three crustal events at Portland hills, Portland hills, Klamath Falls, and Mt. Angels. The results for each scenario seismic event are showed for event are showed for each segment under consideration and are given in Table 7-4 to

Table 7-9. In addition, the casualties for each event and segment are given in

Table C-1 to Table C-24. 


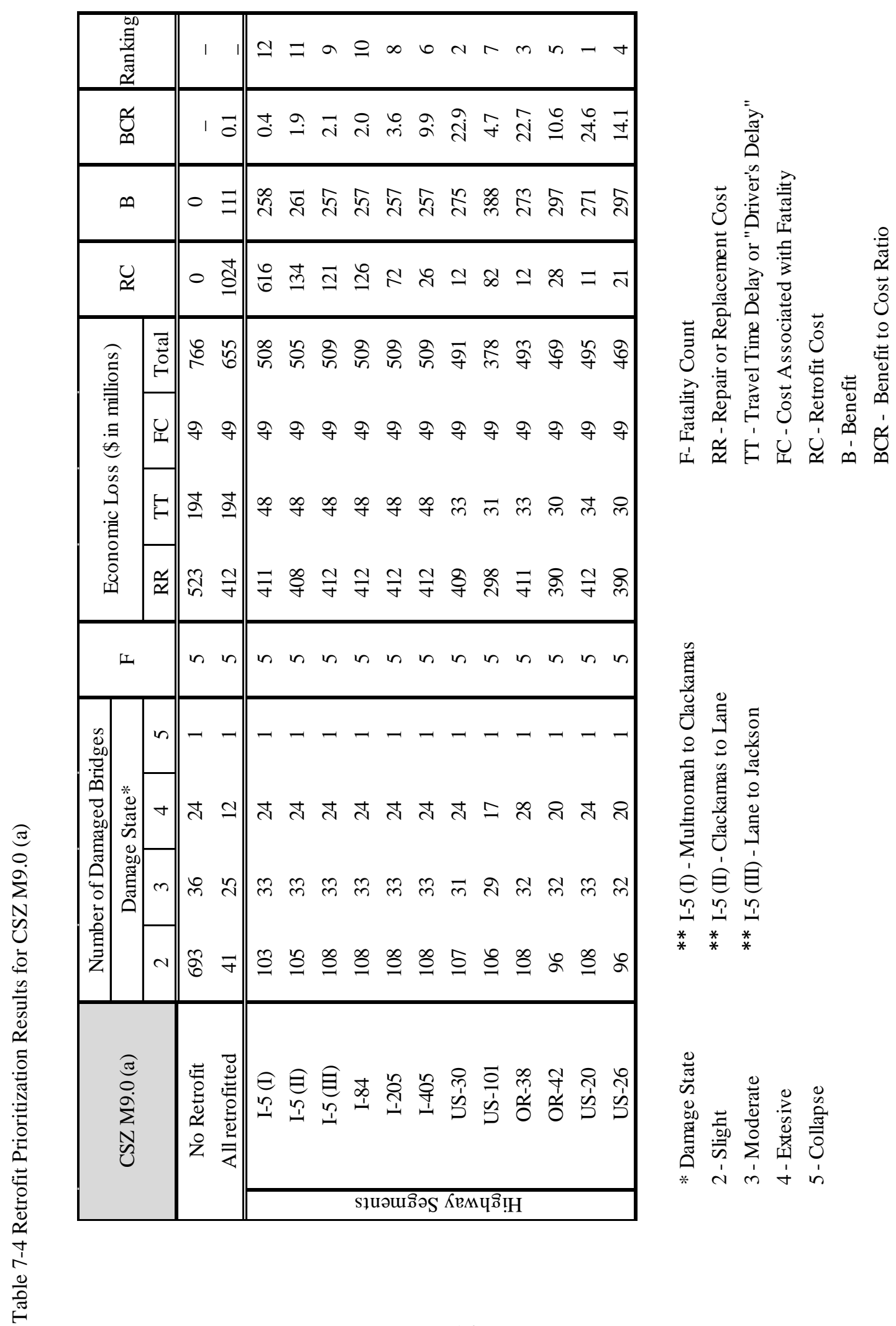




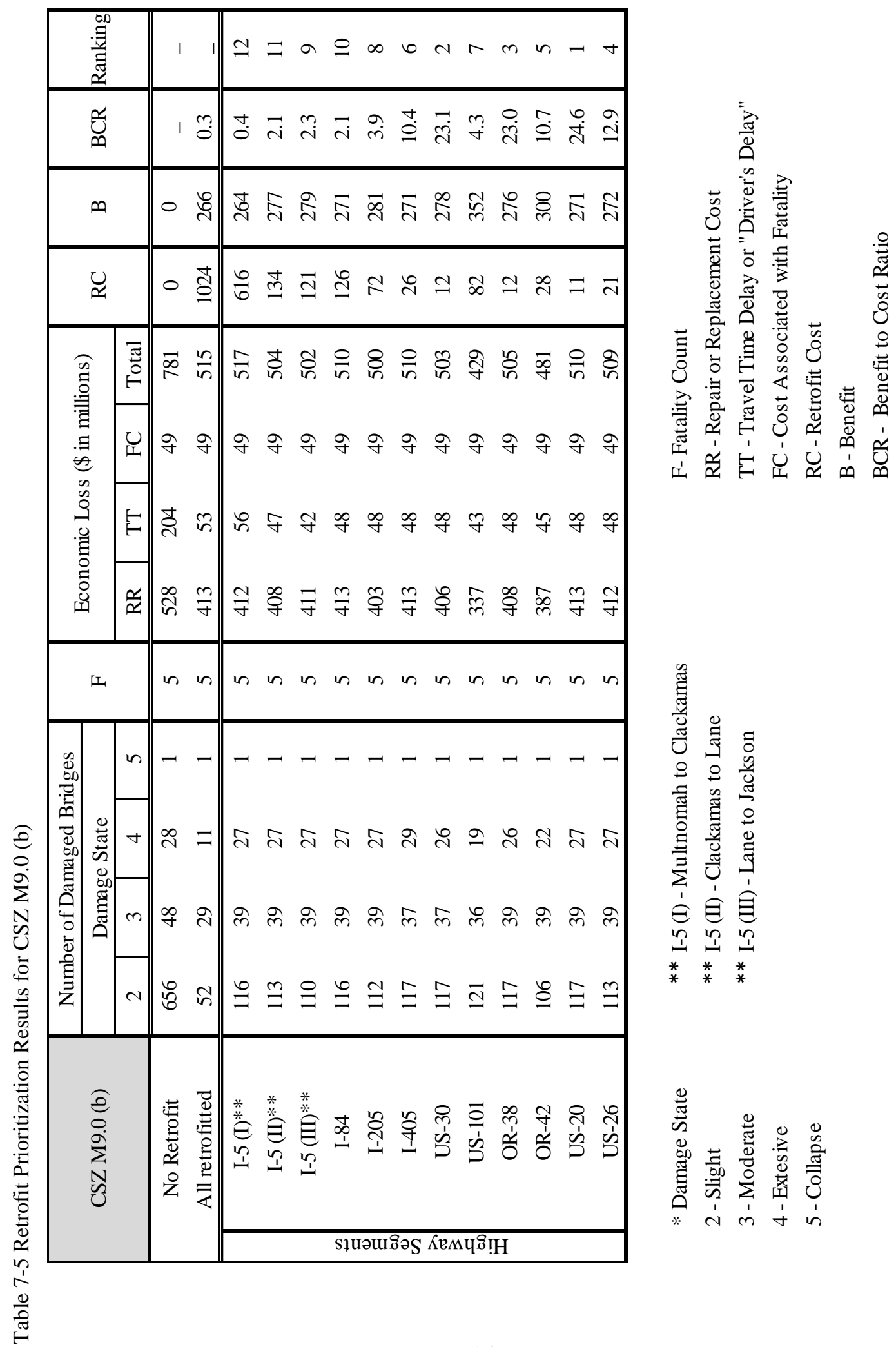




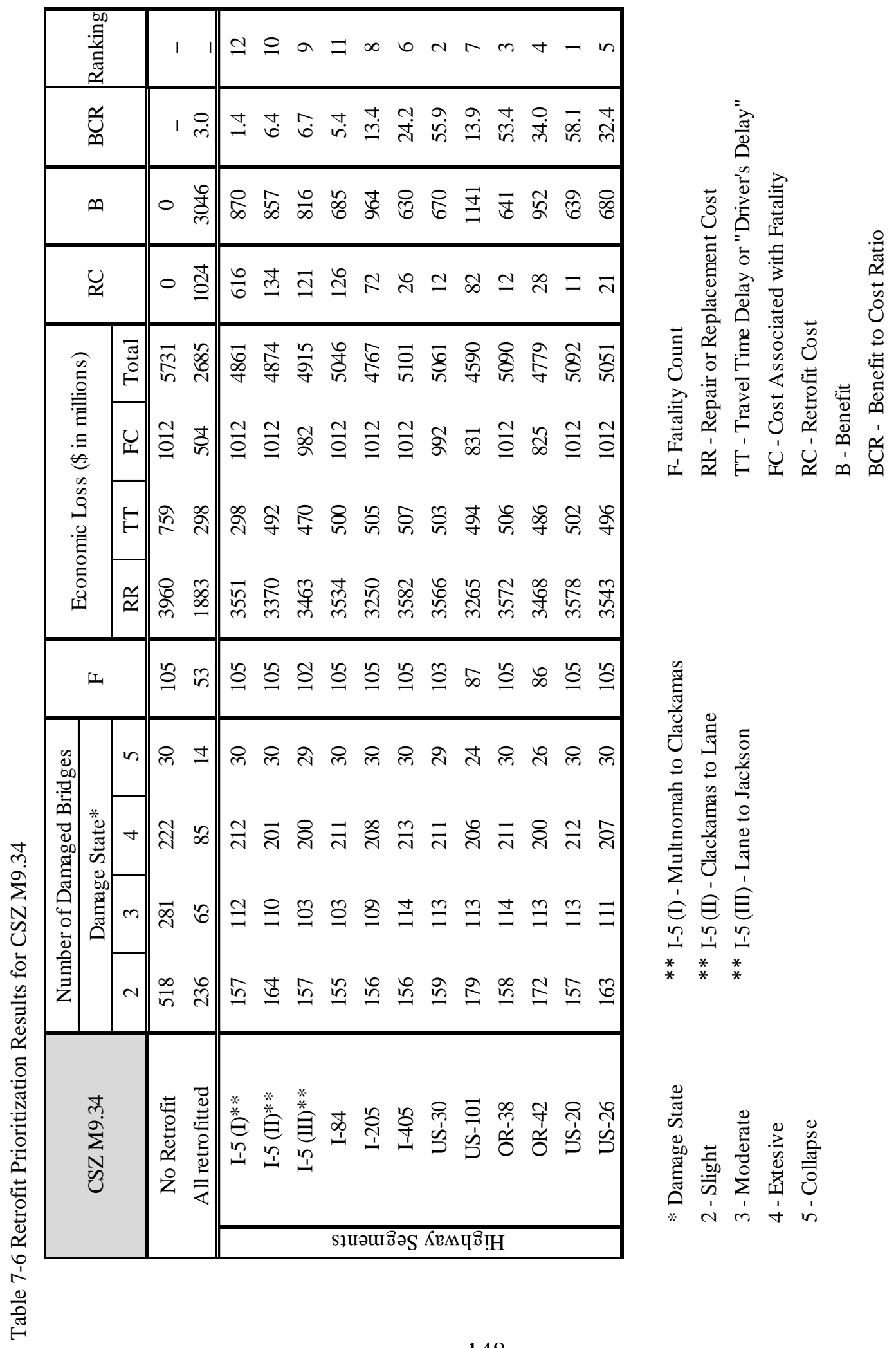




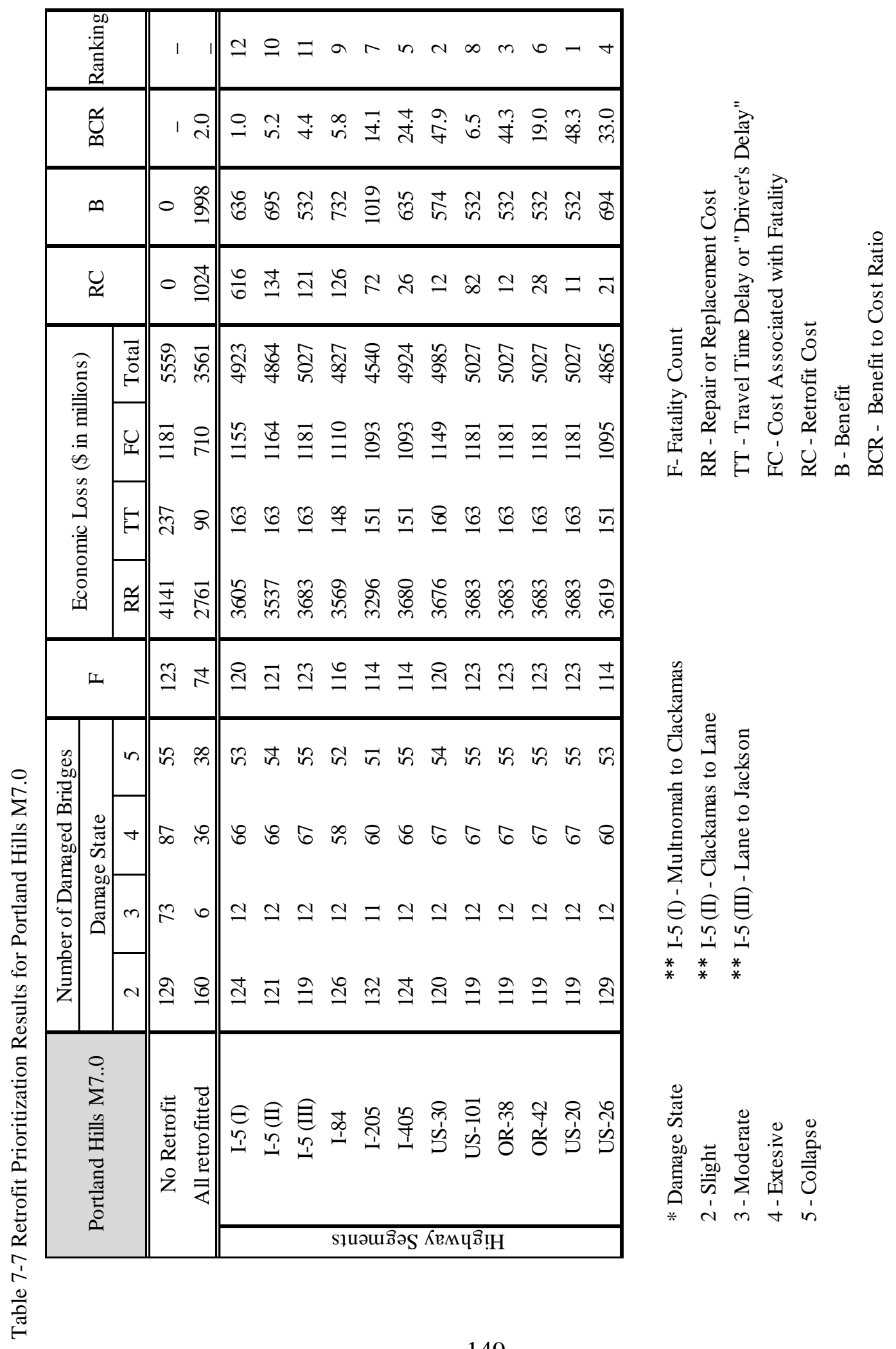




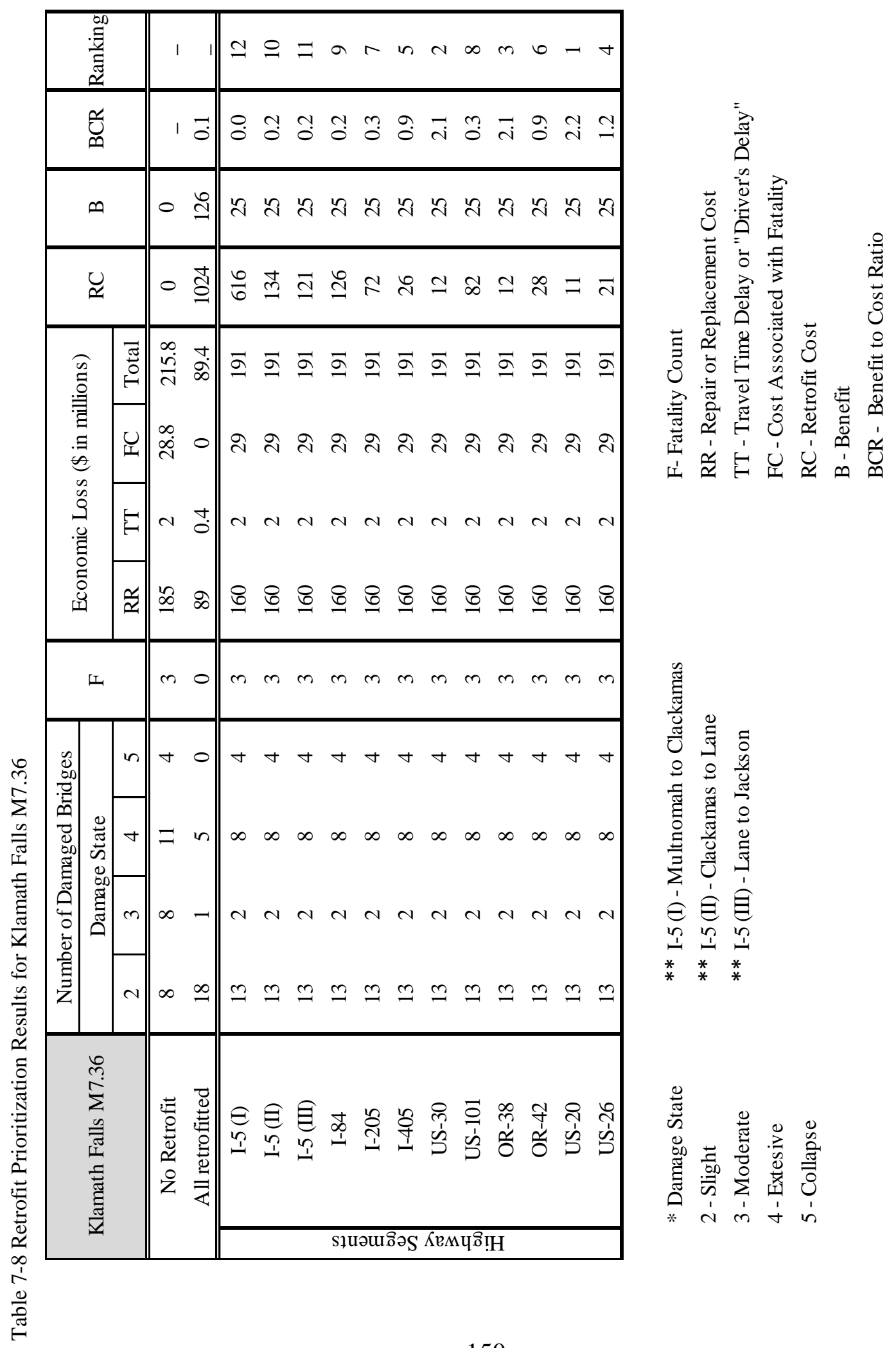




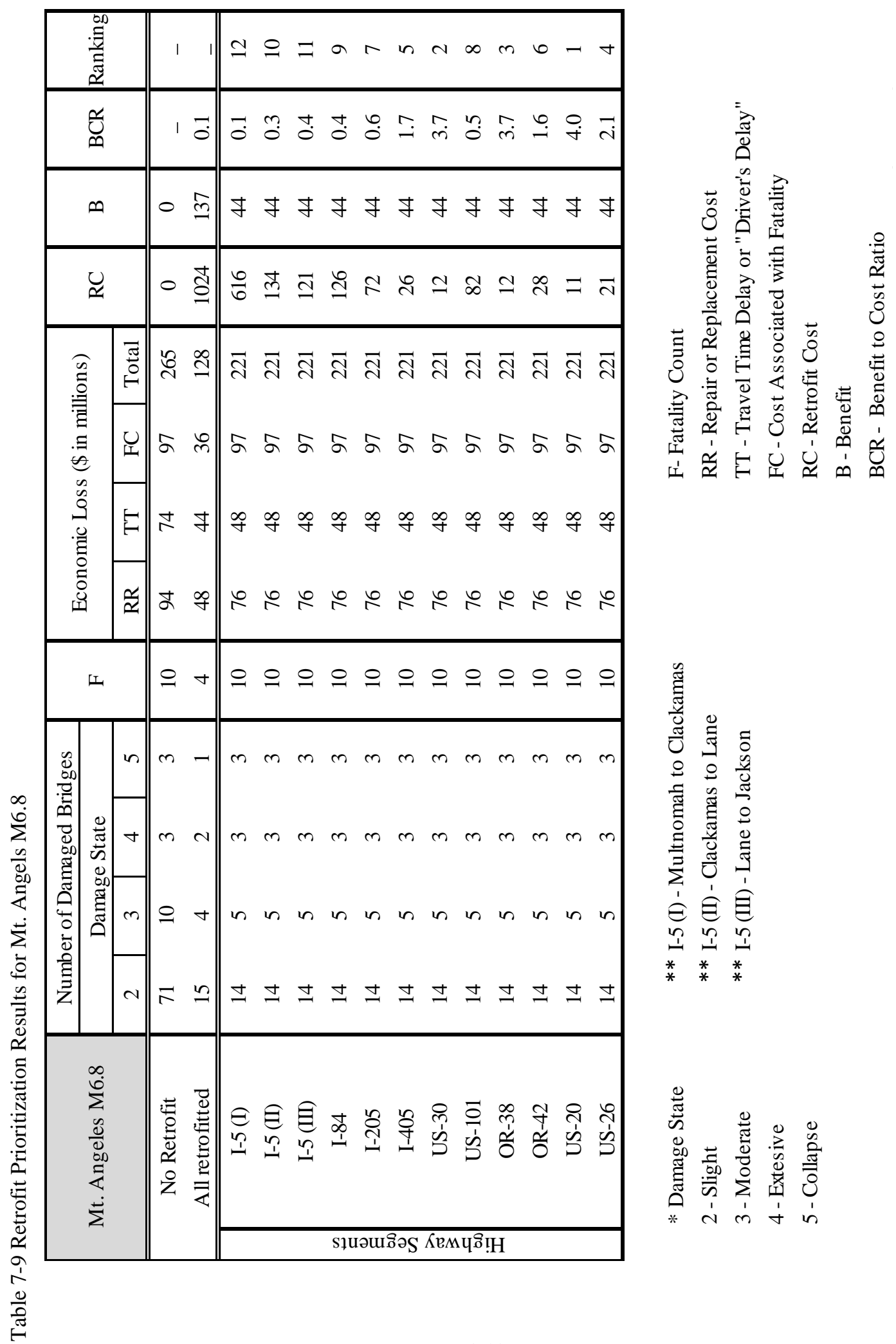




\subsection{Summary}

The results of the above prioritization assessment and associated retrofit cost are summarized in Table 7-10. The result shows that the top five in the ranking are routes located in the East-West corridor connecting I-5 to US-101. The Coast Geographic Zone is the most seismically vulnerable of all the geographic zones and the most difficult to access due to geographic constraints. In addition, because of the high vulnerability of the area, it is very important that that access is maintained for emergency services and recovery. The bridges in these corridors are vulnerable not only to ground shaking, but to landslides, and liquefaction of supporting soils. It should be noted that this study does not include potential landslides and liquefaction that can result due to an earthquake.

However, the framework for prioritization introduced here can be applied for hazards other than ground shaking.

Table 7-10 Summary of Seismic retrofit Prioritization of Routes

\begin{tabular}{c|c|c}
\hline Highway Route & Final Ranking & $\begin{array}{c}\text { Retrofit Cost (\$), } \\
\text { in Million }\end{array}$ \\
\hline \hline US-20 & 1 & 11 \\
OR-38 & 2 & 12 \\
US-30 & 3 & 12 \\
US-26 & 4 & 21 \\
OR-42 & 5 & 28 \\
I-405 & 6 & 26 \\
US-101 & 7 & 82 \\
I-205 & 8 & 72 \\
I-84 & 9 & 126 \\
I-5 (Lane to Jackson) & 10 & 134 \\
I-5 (Clackamas to Lane) & 11 & 616 \\
I-5 (Multnomah to Clackamas) & 12 & 121 \\
\hline
\end{tabular}




\section{CHAPTER 8}

\section{SUMMARY AND CONCLUSIONS}

\subsection{Retrofit Measure}

The experimental study of CFRP retrofitting showed that ductility of bridge columns with lap splice in the plastic hinge zone can be greatly improved the column response. Without the retrofit, similar columns could exhibit shear failure, longitudinal bar buckling and/or lap splice failure. Hence, it can be concluded that applying CFRP wrap is an effective retrofit measure for seismically deficient reinforced concrete square columns with lap splices in the plastic hinge regions. The CFRP helps in preventing bond failures in lap splices by increasing confinement, resulting in just minimal spalling of concrete and longitudinal bar yielding under severe displacement demands. While other retrofit measures may be needed for the overall bridge, cost savings could be realized for the column retrofit aspect.

\subsection{Retrofit Prioritization}

The results from the demonstration case consider the retrofit of only two bridge types; namely, multi-span continuous concrete girder bridge and multi-span simply supported concrete bridge. Consequently, bridge routes that have a low number of these types of bridges may not necessarily be significantly affected by the retrofit of these types of bridges. Furthermore, other devastating effects of earthquakes, such as potential landslides and liquefaction, are not included in these results. However, a similar approach 
can be used to addressing the retrofit of other types of bridges and hazards other than ground shaking for a more comprehensive estimation and ranking

\subsubsection{Limitation in Seismic Risk Assessment Methodology}

Damage states of bridges are computed by first computing the bridge's demand spectral acceleration for a given scenario earthquake, it is then compared to each bridge's spectral acceleration capacity that leads to the onset of each damage state. However, these median values of ground motion computed do not necessarily represent the exact levels of ground shaking at the bridge locations since the exact levels of ground shaking of an earthquake will not be known without actually recording the motion with strong motion accelerometers at the time of the event. Consequently, there is a probability that some bridges might perform better or worse during a real earthquake compared to a scenario analysis.

In addition, fragility values are based on probabilistic median expected performances. A particular bridge that had a specific damage state may not exactly correlate to actual events but is more representative as the expected damage state. For these reasons, the aggregate response over the route should be examined and is more informative than considering the damage state of an individual bridge.

For the social module, the availability of resources to make the repair or replacement, accessibility to bridges and other lifelines, environmental and regional regulations should be studied and applied in the estimation of downtime of a bridge component. 


\subsubsection{Uncertainty in Earthquake Modeling}

It should be noted that a ShakeMap earthquake scenario is a predictive ShakeMap with an assumed magnitude and location, and, optionally, specified fault geometry. The maps give an average effect based on a single possible hypocenter. USGS ShakeMap model use empirical predictive approach to define a median peak-ground-motion value and does not account for variability in motions. And ShakeMap ground motions are more uniform than an actual earthquake that show significant variability.

\subsubsection{Uncertainty in Bridge Modeling}

The vulnerabilities of Oregon bridges are complex and differ from bridge to bridge and from site to site. Therefore, there are uncertainties in bridges' material properties and support types. Moreover, fragility curves should be improved by further studying the use of different bridge bent models with variable geometry and material properties, and also by performing incremental dynamic analysis.

\subsection{Future Work}

Further research is recommended in the following area in order to improve final prioritization ranking

- In order to adequately assess the vulnerability of Oregon's bridges, fragility curves that are specific to Oregon's bridge inventory need to be developed for each bridge class for different levels on damage.

- Numerical models that take into consideration soil effects for foundations should be incorporated into the models. 
- A seismic risk assessment that includes liquefaction and earthquake-induced landslides is necessary to fully understand the vulnerability of the lifeline system.

- A more comprehensive prioritization methodology that takes account of the risk of liquefaction, landslide and other hazards that result due to an earthquake is needed. 


\section{REFERENCES}

Transit New Zealand. (1998). Manual for Seismic Screening of Bridges. Criteria and Guidline manual, Wellington.

AASHTO. (1996). Standard Specifications for Highway Bridges. Washington, D.C.: American Association of State Highway Officials.

AASHTO. (2012). LRFD Bridge Design Specification. American Association of State Highway and Transportation Officials.

Abrahamson, N. A. (1997). Empirical Response Spectral Attenuation Relations for Shallow Crustal Earthquakes. Seismological Research Letters, 94-127.

American Concrete Institute (ACI). (2014). ACI 318 -14 : Building Code Requirements for Reinforced Concrete. American Concrete Institute.

ATC. (1991). Seismic vulnerability and impact of disruption of lifelines in the conterminous United States. Redwood City, CA: ATC.

ATC-13. (1985). Earthquake Damage Evaluation Data for California”. Redwood City, CA : APPLIED TECHNOLOGY COUNCIL.

ATC-6-2. (1983). Seismic Retrofitting Guidlines for Highway Bridges. Redwood, California: Applied technology Council.

Atwater, B. F., B. F., Musumi-Rokkaku, S., K. Satake,, Y., Ueda, K., \& Yamaguchi, D. K. (2005). The orphan tsunami of 1700_Japanese. U.S. Geological Survey. 
Babaei, K., \& Hawkins, N. M. (1993). Bridge Seismic Retrofit Planning Program. Report No. WA-RD 217.1, Washington State Department of Transportation, Olympia, Washington.

Basoz, N., \& Kiremidjian, A. (1996). Risk Assessment for Highway Transportation Systems. PhD. Dissertation submitted to the Department of Civil Engineering, Stanford University.

Basoz, N., \& Kiremidjian, A. (1998). Evaluation of Bridge Damage Data from the Loma Prieta and Northridge, CA Earthquakes. Buffalo, NY.: Multidisciplinary Center for Earthquake Engineering Research.

Basöz, N., \& Kiremidjian, A. S. (1995). Prioritization of Bridges for Seismic Retrofitting. Buffalo, New York: Multidisciplinary Center for Earthquake Engineering.

Basoz, N., \& Mander, J. (1999). Enhancement of the Highway Transportation Lifeline Module in HAZUS. National Institute of Building Sciences.

Bazaez, P., Dusicka, P., Knoles, S., \& Mehary, S. (2015). Bridge Seismic Retrofit Measures Considering Subduction Zone. Portland, OR: ODOT.

Bazaez, R., \& Dusicka, P. (2016). Cyclic Loading for RC Bridge Columns Considering Subduction Megathrust Earthquakes. Journal of Bridge Engineering, 21(5), 04016009. doi:10.1061/(ASCE)BE.1943-5592.0000891

Bazaez, R., \& Dusicka, P. (2018, May). Performance assessment of multi-column RC bridge bents seismically retrofitted with buckling-restrained braces. Bulletin of Earthquake Engineering, 16(5), 2135-2160. 
Berry, M., \& Eberhard, M. (2008). Performance Modeling Strategies for Modern Reinforced Concrete Bridge Columns. University of California, Berkeley: Pacific Earthquake Engineering Research Center, PEER Report 2007/07.

Billah, A., Alam, M., \& Bhuiyan, M. (2013, October). Fragility Analysis of Retrofitted Multicolumn Bridge Bent Subjected to Near-Fault and Far-Field Ground Motion. Journal of Bridge Engineering, 18(10), 992-1004.

Brennan, P. (2004). Background Brief on Bridges. Oregon Legislative Committee Services.

Buckle, I., Friedland, I., Mander, J., Martin, G., Nutt, R., \& Power, M. (2006). Seismic Retrofitting Manual forHighway Structures: Part 1 - Bridges. FHWA.

CH2MHILL. (2012). Seismic Lifelines Evaluation, Vulnerability Synthesis, and Identification. Corvallis, OR: ODOT.

Chai, Y. H., Priestley, M. J., \& Seible, F. (1991). Retrofit of Bridge Columns for Enhanced Seismic Performance. Seismic Assessment and Retrofit of Bridges(SSRP 91/03), pp. 177-196.

Cherng, R., \& Wen, Y. K. (1992). Reliability Based Cost-Effective Retrofit of Highway Transportation Systems Against Seismic Hazard. University of Illinois, Civil Engineering.

Choi, E., DesRoches, R., \& Nielson, B. (2003). Seismic fragilityof typical bridges in moderate seismic zones. Engineering Structures, 187-199. 
Coburn, A., \& Spence, R. (1992). Factors Determining Human Casualty Levels in. Proceedings of the 10 WCEE, (pp. 5989 - 5994). Madrid, Spain.

Dunwoodie, D. (1997). Seismic Retrofit of Bridge Columns Using Fibre-reinforced Concrete. McGill University, Department of Civil Engineering and Applied Mechanics. McGill University: National Library of Canada.

Durkin, M., \& Thiel, C. (1991). Integrating Earthquake Casualty and Loss. Proc. of the Workshop on Modeling Earthquake Casualties for Planning. Sacramento.

Dusicka, P., Glickman, M., \& Oppenheimer, H. (2007). “Seismic Vulnerability of Oregon Highway Bridges. final report to Oregon Department of Transportation, Salem, OR.

Dutta, , A., \& Mander, J. B. (1999). Seismic fragility analysis of highway bridges. Centerto-Center Project Workshop on Earthquake Engineering in Transportation Systems. Tokyo, Japan.

ElGawady, M., Endeshaw, M., McLean, D., \& Sack, R. (2010). Retrofitting of Rectangular Columns with Deficient Lap Splices. Journal of Composites for Construction, ASCE, 14(1), 22-35.

Elwood, K., \& Eberhard, M. (2009). Effective Stiffness of Reinforced Concrete Columns. ACI Structural Journal, 106(4), 476-484.

Endeshaw, M., ElGawady, M., Sack, R. L., \& McLean, D. I. (2008). Retrofit of Rectangular Bridge Columns Using CFRP Wrapping. Pullman, WA: Washington State Transportation Center (TRAC). 
Enke, D., Tirasirichai, C., \& Luna, R. (2008). Estimation of Earthquake Loss due to Bridge Damage in the St. Louis Metropolitan Area: Part II - Indirect Losses. Natural Hazards Review, 9(1), 12-19.

FEMA. (2010). HAZUS MR4 Multi-hazard Loss Estimation Methodology. Washington D.C.: Federal Emergency Management Agency.

FHWA. (2006). Seismic Retrofitting Manual for Highway Structures: Part 1 - Bridges. Buffalo, NY: FHWA.

Goodnight, J. C., Kowalsky, M. J., \& Nau., J. M. (2013). Effect of Load History on Performance Limit States of Circular Bridge Columns. Journal of Bridge Engineering, 18(12), 1383-1396.

Grossi, P. (2000). Earthquake Damage Assessment From Expert Opinion to Fragility Curves. 8th ASCE Specialty Conference on Probabilistic Mechanics and Structural Reliability. Notre Dame, IN.

Gulkan, P., \& Sozen, M. (1974). Inelastic responses of reinforced concrete structures to earthquake motions. Journal of the American Concrete Institute, 71(12), 604-610.

Haroun, M. A., \& Elsanadedy, H. M. (2005). Fiber-Reinforced Plastic Jackets for Ductility Enhancement of Reinforced Concrete Bridge Columns with Poor Lap-Splice Detailing. Journal of Bridge Engineering, 10(6), 749-757. Retrieved May 4, 2012, from http://ascelibrary.org/beo/resource/1/jbenf2/v10/i6/p749_s1?view=fulltext

Hines, E. M., Seible, F., \& Priestley, M. N. (2002). Seismic Performance of Hollow Rectangular Reinforced Concrete Bridge Piers with Highly-Confined Corner 
Elements; Phase I Flexural Tests, Phase II Shear Tests. San Diego, CA: University of California, San Diego.

Hwang, H., Jernigan, J. B., \& Lin, Y. (1999). Expected Seismic Damage to Memphis Highway Systems. 5th U.S. Conference on Lifeline Earthquake Engineering (pp. 1-10). Seattle, WA: American Association of Civil Engineers.

Hwang, H., Jernigan, J. B., \& Lin, Y. (2000). Evaluation of seismic damage to memphis bridges and highway systems. Journal of Brdge Engineering, 322-330.

Hwang, H., Liu, J. B., \& Chiu, Y.-H. (2001). Seismic Fragility Analysis of Highway Bridges. Memphis: Center for Earthquake Research and Information.

Karim, K. R., \& Yamazaki, F. (2001). Effect of earthquake ground motions on fragility curves of highway bridge piers based on numerical simulation. Earthquake Engineering and Structural Dynamics, 30, 1839-1856.

Karim, K. R., \& Yamazaki, F. (2003). A simplified method of constructing fragility curves for Highway Bridges. Earthquake Engineering and Structural Dynamics, 32, 16031626.

Karim, K., \& Yamazaki, F. (2000). Comparison of Analytical Fragility Curves for RC Bridge Piers in Japan. 8th ASCE Specialty Conference on Probabilistic Mechanics and Structural Reliability,

Kim, S. H., \& Shinozuka, M. (2004). Development of fragility curves of bridges retrofitted by column jacketing. Probabilistic Engineering Mechanics, 105-112. 
Kim, S., Gaus, M., Lee, G., \& Chang, K. (1992). A GIS-Based Regional Risk Approach for Bridges Subjected to Earthquakes. In proceedings of 8th Conference on Computing in Civil Engineering and Geographical Information System Symposium, (pp. 460-467). Texas.

King, S. A., Kiremidjian, A. S., Basoz, N., Law, K., Vucetic, M., Doroudian, M., ... . Horner, G. (1997, NOvember). Methodologies for Evaluating the Socio-Economic Consequences of Large Earthquakes. Earthquake Spectra, 13(4), 565-584.

Lee, G. C., Mohan, S. B., Haung, C., \& Fard, B. N. (2013). A Study of U. S. Bridge Failures (1980-2012). U.S. Department of Transportation Federal Highway Administration. University at Buffalo, State University of New York: MCEER . Retrieved from <https://mceer.buffalo.edu/pdf/report/13-0008.pdf $>$.

Liao, W.-I., \& Loh, C.-H. (2004). Priliminary Study on the Fragility Curves for Highway Bridges in Taiwan. Journal of the Chinese Institute of Engineers, 27(3), 367-375.

Mander, J., \& Basöz, N. (1999). Enhancement of the Highway Transportation Lifeline Module in HAZUS. NIBS.

Mander, J., Priestley, M., \& Park, R. (1988). Theoretical Stress-Strain Model for Confined Concrete. Journal of Structural Engineering, 114(3), 1804-1826.

McLean, D. I., \& Bernards, L. L. (1992). Seismic Retrofitting of Rectangular Bridge Column for Shear. Pulman, WA: Washington State Transportation Center (TRAC).

Mehary, S., \& Dusicka, P. (2012). Seismic hazard Assessment of Oregon HIghway Truck Routes. Portland: OTREC. 
Mehary, S., Dusicka, P., \& Bazaez, R. (2017). Effect of Subduction Earthquake-Based Loading History on Substandard. Journal of Bridge Engineering, 23(3), 04017146.

Moehle, J. (2015). Seismic Deisgn of Reinforced Concrete Buildings. McGraw Hill Education.

Moore, J. E., Cho, S., Fan, Y. Y., \& Werner, S. (2006). Quantifying Economic Losses from Travel Forgone Following a Large Metropolitan Earthquake. Berkeley: PEER.

Murat, S., \& Cem, Y. (1998-03-24). Canada/Ottawa Patent No. US6247279B1.

Nako, A., Shlke, C., Six, J., Johnsom, B., Dusicka, P., \& Mehary, S. (2009). Seismic Vulnerability of Oregon State Highway Bridges: Mitigation Strategies. Salem, OR: ODOT.

Nielson, B. (2005). Analytical Fragility Curves for Highway Bridges in Moderate Seismic Zones. Ph.D. thesis. Atlanta: Georgia Institute of Technology.

Nielson, B. G., \& DesRoches, R. (2007, August). Analytical Seismic Fragility Curves for Typical Bridges in the Central and Southeastern United States. Earthquake Spectra, 23(3), 615-633.

ODOT. (2009). Seismic Vulnerability of Oregon State Highway Bridges: Mitigation Strategies To Reduce Major Mobility Risks. Bridge Engineering Section. Salem, OR: ODOT.

ODOT. (2013). Oregon Highways Seismic Options Report. Bridge and Geo-Environmental Sections Technical Services Branch. ODOT. 
OpenSees. (2013). Open System for Earthquake Engineering Simulation, Pacific Earthquake Engineering Research Center, University of California, Berkeley V. 2.5.0. Retrieved from http://opensees.berkeley.edu

OSSPAC. (2013). The Oregon Resilience Plan - Reducing Risk and Improving Recovery for the Next Cascadia Earthquake and Tsunami. Salem.

Padgett, J. (2005, July). Retrofitted Bridge Fragility Curves for Assessing the Consequences of an Earthquake Event. Retrieved December 11, 2010, from MidAmerica Earthquake Center: http://mae.cee.uiuc.edu/documents/slc_online_magazine_2005_july_padgett.pdf

Padgett, J. E. (2010). Sustainability as a Guide for Selecting and Prioritizing Seismic Sustainable Bridges. 2010 Concrete Bridge Conference. Phoenix.

Padgett, J. E., \& DesRoches, R. (2007, December). Sensitivity of Seismic Response and Fragility to Parameter Uncertainty. Journal of Structural Engineering, 1710-1718.

Padgett, J. E., \& DesRoches, R. (2008, April 11). Methodology for the development of analytical fragility curves for. Earthquake Engineering and Structural Dynamics, 37, 1157-1174.

Padgett, J. E., \& DesRoches, R. (2009). Retrofitted Bridge Fragility Analysis for Typical Classes of Multispan Bridges. Earthquake Spectra, 117-141.

Pan, Y. (2007). Seismic Fragility and Risk Management of Highway Bridges in New York State. New York: The City University of New York. 
Priestley, M. J., Calvi, G. M., \& Kowalsky, M. J. (2007). Displacement-based seismic design of structures,. (I. Press, Ed.) Pavia, Italy.

Priestley, M., Seible, F., \& Calvi, G. (1996). Seismic Design and Retrofit of Bridges. New York: John Wiley \& Sons, Inc.

Roberts, J. (1991). Recent Advances in Seismic Design and Retrofit of California Bridges. In Proceedings of the 3rd U.S. National Conference on Lifeline Earthquake Engineering., (pp. 52-64). Los Angeles, California.

Rodriguez, M., \& Park, R. (1994, Mar-Apr). Seismic Load Test on Reinforced Concrete Columns Strengthened by Jacketing. ACI Structural Journal, 91(2), 150-159.

Roy, N., Paultre, P., \& Proulx, J. (2010). Performance based seismic retrofit of a bridge bent: Design and experimental validation. Can. J. Civ. Eng, 37, 367-379.

Saatcioglu, M., \& Yalcin, C. (2003). External Prestressing Concrete Columns for Improved Seismic Shear Resistance. Journal of Structural Engineering, 1057-1070.

Scott, M., \& Fenves, G. (2006). Plastic Hinge Integration Methods for Force-Based BeamColumn Elements. Journal of Structural Engineering, 132(2), 244-252. doi:10.1061/(ASCE)0733-9445(2006)132:2(244)

Seible, F., Hegemier, G., Priestley, M., Innamorato, D., \& Ho, F. (1995). Carbon Fiber Jacket Retrofit Test of Rectangular Flexural Column with Lap Spliced Reinforcement. University of California, San Diego: Advanced Composites Technology Transfer Consortium Report No. ACTT-95/04. 
Seville, E., \& Metcalfe, J. (2005). Developing a hazard risk assessment Framework for the New Zealand State Highway Network. Land Transport New Zealand.

Shinozuka, M., Feng, M. Q., Kim, H., \& Kim, S. (2000, December). Nonlinear Static Procedure for Fragility Curve Develipment. Journal or Engineering Mechanics, 1287-1295.

Shinozuka, M., Feng, M. Q., Lee, J., \& Naganuma, T. (2000, December). Statistical Analysis of Fragility Curves. Journal of Engineering Mechanics, 1224-1231.

Shinozuka, M., Feng, M., Kim, H., \& Kim, S. (2000). Nonlinear Static Procedure for Fragility Curve Development. Journal or Engineering Mechanics (ASCE), 126(12), 1287-1295.

Shinozuka, M., Kim, S., Kushiyama, S., \& Yi, J. (2002, December). Fragility Curves of Concrete Bridges Retrofitted By Column Jacketing. Earthquake Engineering and Engineering Vibration, 1(2), 195-202.

Shinozuka, M., Saxena, V., Deodatis, G., \& Feng, M. Q. (2001, September 17). Development of Fragility Curves for Multi-Span Reinforced Concrete Bridges. Retrieved September 30, 2010, from http://www.civil.columbia.edu/iassar/: http://www.civil.columbia.edu/iassar/downloadfiles/SC1_texts/

Shinozuka, M., Sgaravato, M., \& Banerjee, S. (2008). Optiaml Seismic Bridge Retrofit Strategy Under Budget Constraint. The 14th World Conference on Earthquake Engineering. Beijing, Chhina. 
Shinozuka, M., Shiraki, N., \& Kameda, H. (2000). Performance of Highway Network Systems under Earthquake Damage. Proceedings of the Second International Workshop on Mitigation of Seismic Effects on Transportation Structures, (pp. 303317). Taiwan.

Simon, J., Bracci, J. M., \& Gardoni, P. (2010, October). Seismic Response and Fragility of Deteriorated Reinforced Concrete Bridges. Journal of Structural Engineering, $1273-1281$.

Stevanovic, A., \& Nadimpalli, P. (2010). Seismic Vulnerability and Emergency Response Analyses of UDOT Lifelines. Department of Civil \& Environmental Engineering, University of Utah.

Stojadinović, B., \& Mackie, K. (2007, July/August). R-Factor Parameterized Bridge Damage Fragility Curves. Journal of Bridge Engineering, 500-510.

Taucer, F., Spacone, E., \& Filippou, F. (1991). A fiber beam-column element for seismic response analysis of reinforced concrete structures. Berkeley, CA: Earthquake Engineering Research Center, College of Engineering, University of California. Report 91-17.

U. S. Department of Transportation. (2007). Treatment of the economic value of a V. Washington, D.C.: Office of Assistant Secretary for.

U. S. Department of Transportation. (2016, August 8). Treatment of the Value of Preventing Fatalities and Injuries in Preparing Economic Analyses. 
U.S. Census Bureau. (2018, May 1). Oregon 2010 Census Tracts. Retrieved from Oregon Spatial

Data

Library: http://navigator.state.or.us/sdl/data/2010census/tracts2010.zip

Wang, E. (2006). Optimizing Bridge Seismic Retrofit Strategy Implementing Bridge Fragility Curves. STRUCTURES.

Werner, S. D., \& Taylor, C. E. (2002). Component vulnerability modeling issues for analysis of seismic risks to transportation lifeline systems. ASCE.

Werner, S. D., Taylor, C. E., Cho, S., Lavoie, J., Huyck, C., Eitzel, C., . . Eguchi, E. T. (2006). Technical Manual: REDARS 2 Methodology and Software for Seismic Risk Analysis of Highway Systems. Oakland.

Werner, S. D., Taylor, C. E., Cho, S., Lavoie, J.-P., Huyck, C. K., Eitzel, C., . . II, J. E. (2004). New Developments in Seismic Risk Analysis of Highway Systems. 13th World Conference on Earthquake Engineering. Vancouver, B.C., Canada.

Whitman, R. V., Brennan III, J. M., Cornell, C. A., de Neufville, R. L., \& Vanmarcke, E. H. (1975). Seismic Design Decision Analysis. Journal of the Structural Division, 101(ST5), 1067-1084.

Woodward-Clyde Consultants. (1991). Report of Seismic Bridge Condition Survey. Vol. 1, (Prepared for Illinois Dept. of Transportation), Oakland, California.

Wright, T., Desroches, R., \& Padgett, J. E. (2010). Bridge Seismic Retrofitting Practices in the Central and Southeastern United States. Journal of Bridge Engineering, 8292. 
WSDOT. (2006). Bridge Design Manual M 23-50.

Yamazaki, F., Ohnishi, J., \& Tayama, S. (1999). Earthquake Damage Assessment of Expressway Structures in Japan. Asia-Pacific Symposium on Structural Reliability and its Application, (pp. 205-214). Taipei, Taiwan. 


\section{APPENDIX A. SPECIMEN PREPARATION}
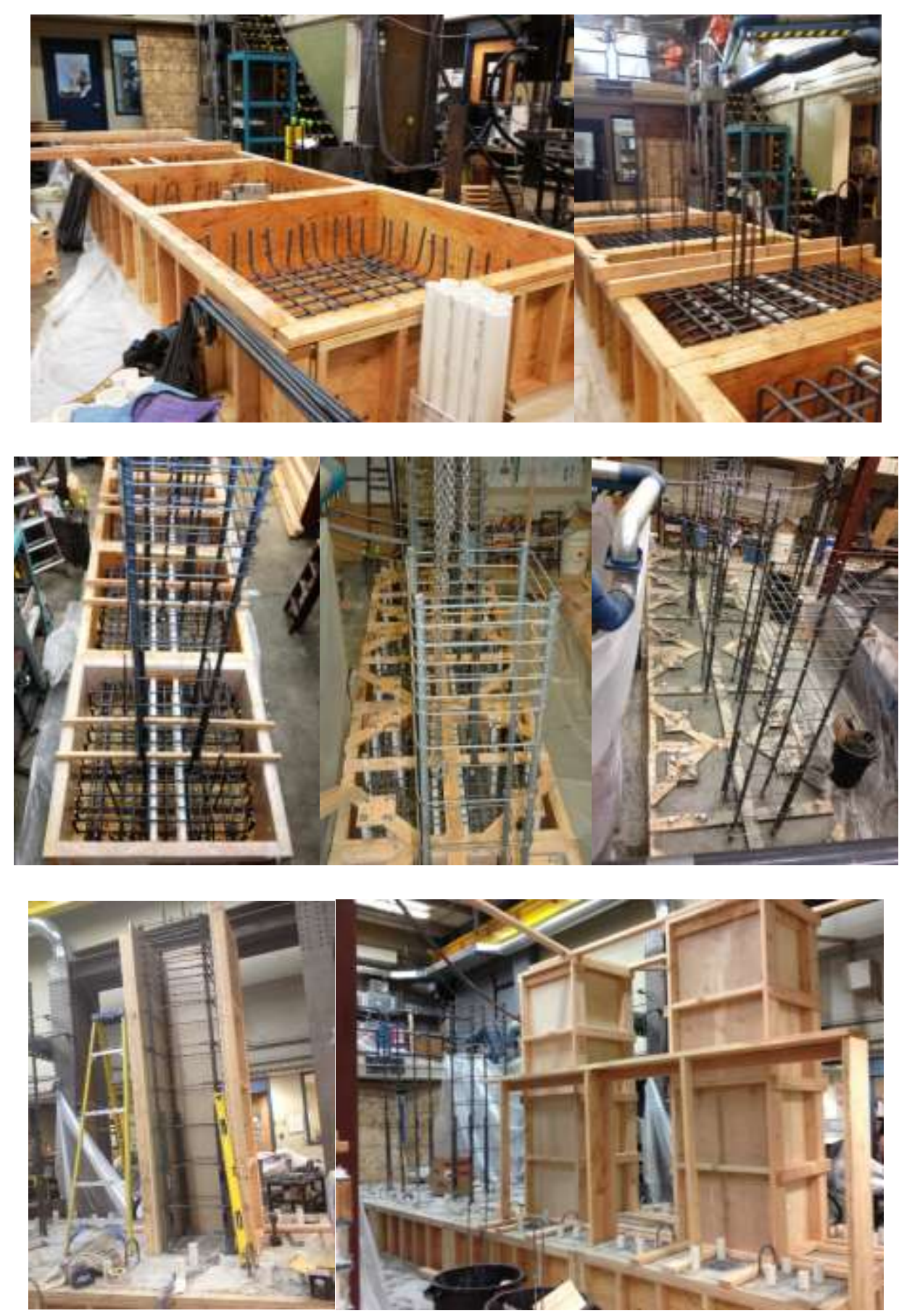

Figure 0-1 Specimen Preparation - Formwork and Rebar Placement 


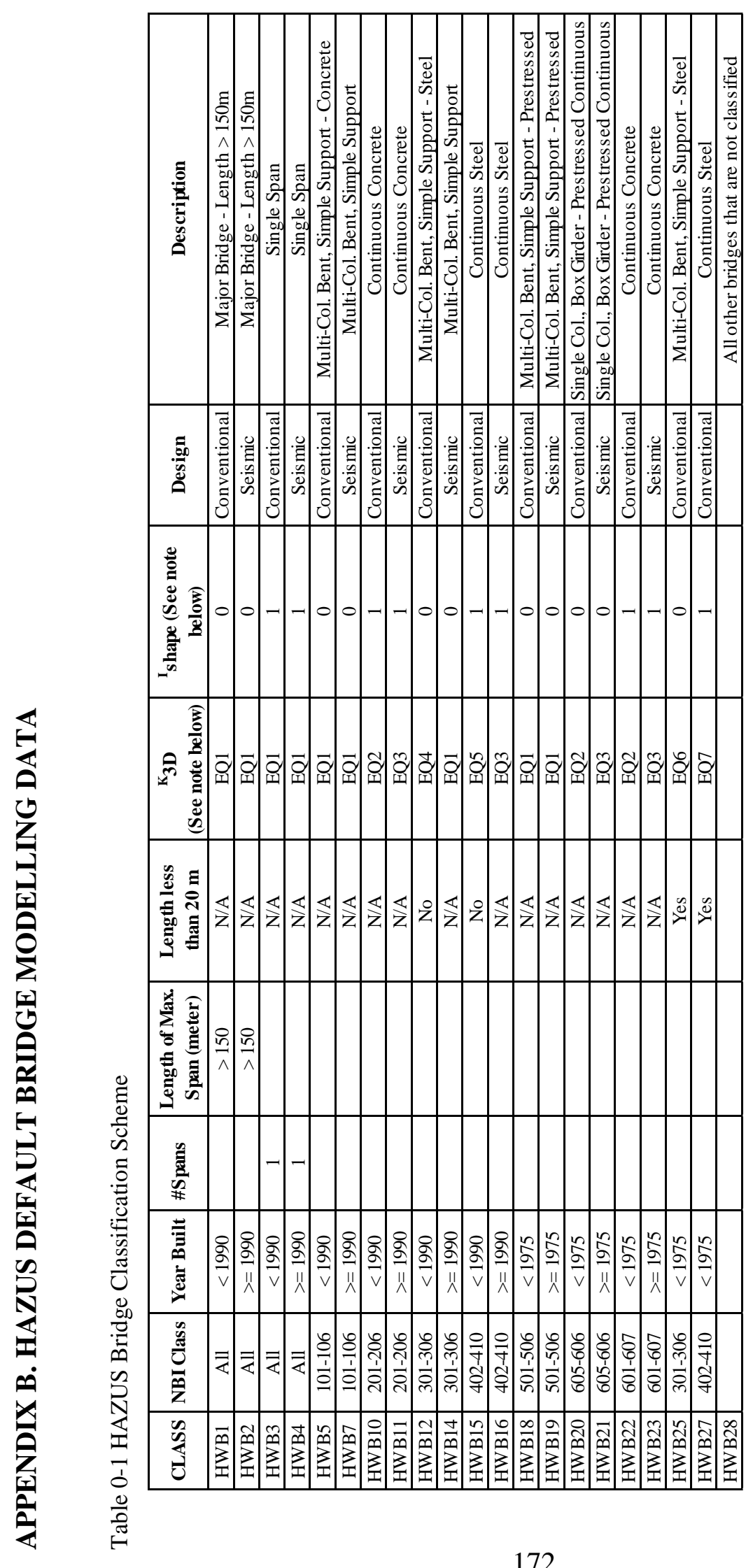

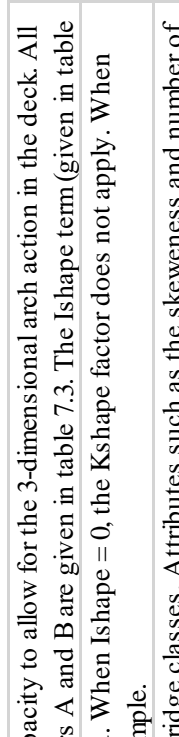

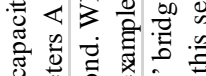

范产

음 흥으은

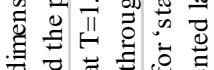

氜

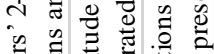

. 츨 흘

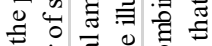

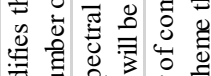

의

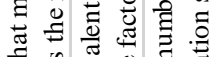

足空

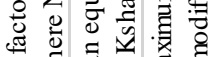

牥

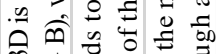

21100

बิ

on 4 क

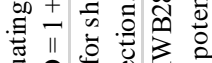

피웡

匀

苛

w 웡

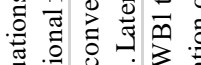

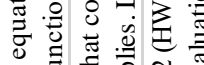

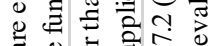

근

의

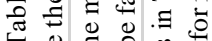

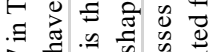

oิ

들

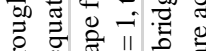

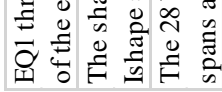


Table 0-2 Coefficients for Evaluating $\mathrm{K}_{3 \mathrm{D}}$

\begin{tabular}{|c|c|c|c|}
\hline Equation & $\mathbf{A}$ & $\mathbf{B}$ & $\mathbf{K}_{3 \mathbf{3}}$ \\
\hline EQ1 & 0.25 & 1 & $1+0.25 /(\mathrm{N}-1)$ \\
\hline EQ2 & 0.33 & 0 & $1+0.33 /(\mathrm{N})$ \\
\hline EQ3 & 0.33 & 1 & $1+0.33 /(\mathrm{N}-1)$ \\
\hline EQ4 & 0.09 & 1 & $1+0.09 /(\mathrm{N}-1)$ \\
\hline EQ5 & 0.05 & 0 & $1+0.05 /(\mathrm{N})$ \\
\hline EQ6 & 0.20 & 1 & $1+0.20 /(\mathrm{N}-1)$ \\
\hline EQ7 & 0.10 & 0 & $1+0.10 /(\mathrm{N})$ \\
\hline
\end{tabular}


Table 0-3 Damage Algorithms for Bridges

\begin{tabular}{|c|c|c|c|c|c|c|c|c|}
\hline CLASS & Slight & Moderate & Extensive & Complete & Slight & Moderate & Extensive & Complete \\
\hline HWB1 & 0.40 & 0.50 & 0.70 & 0.90 & 3.9 & 3.9 & 3.9 & 13.8 \\
\hline HWB2 & 0.60 & 0.90 & 1.10 & 1.70 & 3.9 & 3.9 & 3.9 & 13.8 \\
\hline HWB3 & 0.80 & 1.00 & 1.20 & 1.70 & 3.9 & 3.9 & 3.9 & 13.8 \\
\hline HWB4 & 0.80 & 1.00 & 1.20 & 1.70 & 3.9 & 3.9 & 3.9 & 13.8 \\
\hline HWB5 & 0.25 & 0.35 & 0.45 & 0.70 & 3.9 & 3.9 & 3.9 & 13.8 \\
\hline HWB6 & 0.30 & 0.50 & 0.60 & 0.90 & 3.9 & 3.9 & 3.9 & 13.8 \\
\hline HWB7 & 0.50 & 0.80 & 1.10 & 1.70 & 3.9 & 3.9 & 3.9 & 13.8 \\
\hline HWB8 & 0.35 & 0.45 & 0.55 & 0.80 & 3.9 & 3.9 & 3.9 & 13.8 \\
\hline HWB9 & 0.60 & 0.90 & 1.30 & 1.60 & 3.9 & 3.9 & 3.9 & 13.8 \\
\hline HWB10 & 0.60 & 0.90 & 1.10 & 1.50 & 3.9 & 3.9 & 3.9 & 13.8 \\
\hline HWB11 & 0.90 & 0.90 & 1.10 & 1.50 & 3.9 & 3.9 & 3.9 & 13.8 \\
\hline HWB12 & 0.25 & 0.35 & 0.45 & 0.70 & 3.9 & 3.9 & 3.9 & 13.8 \\
\hline HWB13 & 0.30 & 0.50 & 0.60 & 0.90 & 3.9 & 3.9 & 3.9 & 13.8 \\
\hline HWB14 & 0.50 & 0.80 & 1.10 & 1.70 & 3.9 & 3.9 & 3.9 & 13.8 \\
\hline HWB15 & 0.75 & 0.75 & 0.75 & 1.10 & 3.9 & 3.9 & 3.9 & 13.8 \\
\hline HWB16 & 0.90 & 0.90 & 1.10 & 1.50 & 3.9 & 3.9 & 3.9 & 13.8 \\
\hline HWB17 & 0.25 & 0.35 & 0.45 & 0.70 & 3.9 & 3.9 & 3.9 & 13.8 \\
\hline HWB18 & 0.30 & 0.50 & 0.60 & 0.90 & 3.9 & 3.9 & 3.9 & 13.8 \\
\hline HWB19 & 0.50 & 0.80 & 1.10 & 1.70 & 3.9 & 3.9 & 3.9 & 13.8 \\
\hline HWB20 & 0.35 & 0.45 & 0.55 & 0.80 & 3.9 & 3.9 & 3.9 & 13.8 \\
\hline HWB21 & 0.60 & 0.90 & 1.30 & 1.60 & 3.9 & 3.9 & 3.9 & 13.8 \\
\hline HWB22 & 0.60 & 0.90 & 1.10 & 1.50 & 3.9 & 3.9 & 3.9 & 13.8 \\
\hline HWB23 & 0.90 & 0.90 & 1.10 & 1.50 & 3.9 & 3.9 & 3.9 & 13.8 \\
\hline HWB24 & 0.25 & 0.35 & 0.45 & 0.70 & 3.9 & 3.9 & 3.9 & 13.8 \\
\hline HWB25 & 0.30 & 0.50 & 0.60 & 0.90 & 3.9 & 3.9 & 3.9 & 13.8 \\
\hline HWB26 & 0.75 & 0.75 & 0.75 & 1.10 & 3.9 & 3.9 & 3.9 & 13.8 \\
\hline HWB27 & 0.75 & 0.75 & 0.75 & 1.10 & 3.9 & 3.9 & 3.9 & 13.8 \\
\hline HWB28 & 0.80 & 1.00 & 1.20 & 1.70 & 3.9 & 3.9 & 3.9 & 13.8 \\
\hline
\end{tabular}




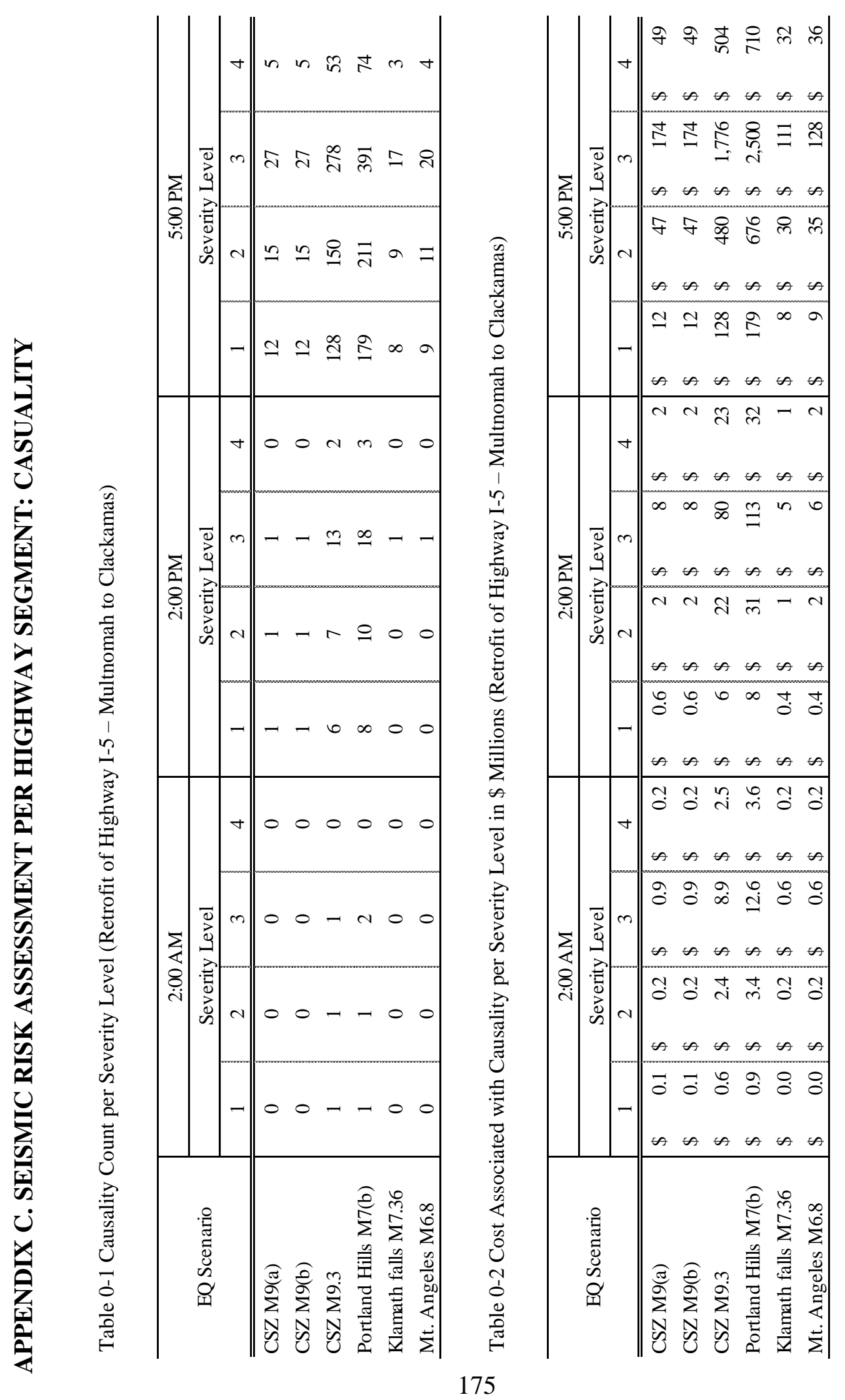



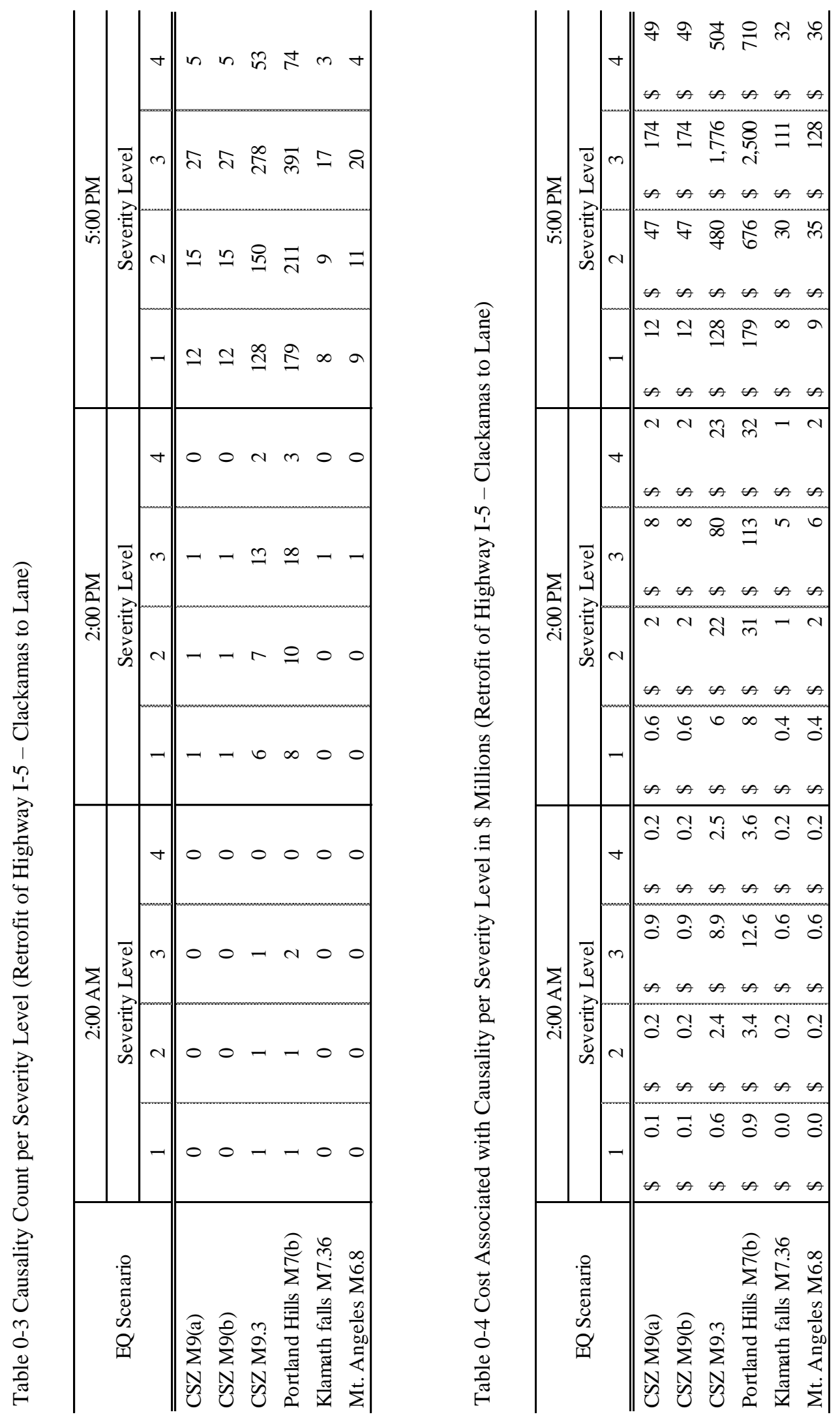

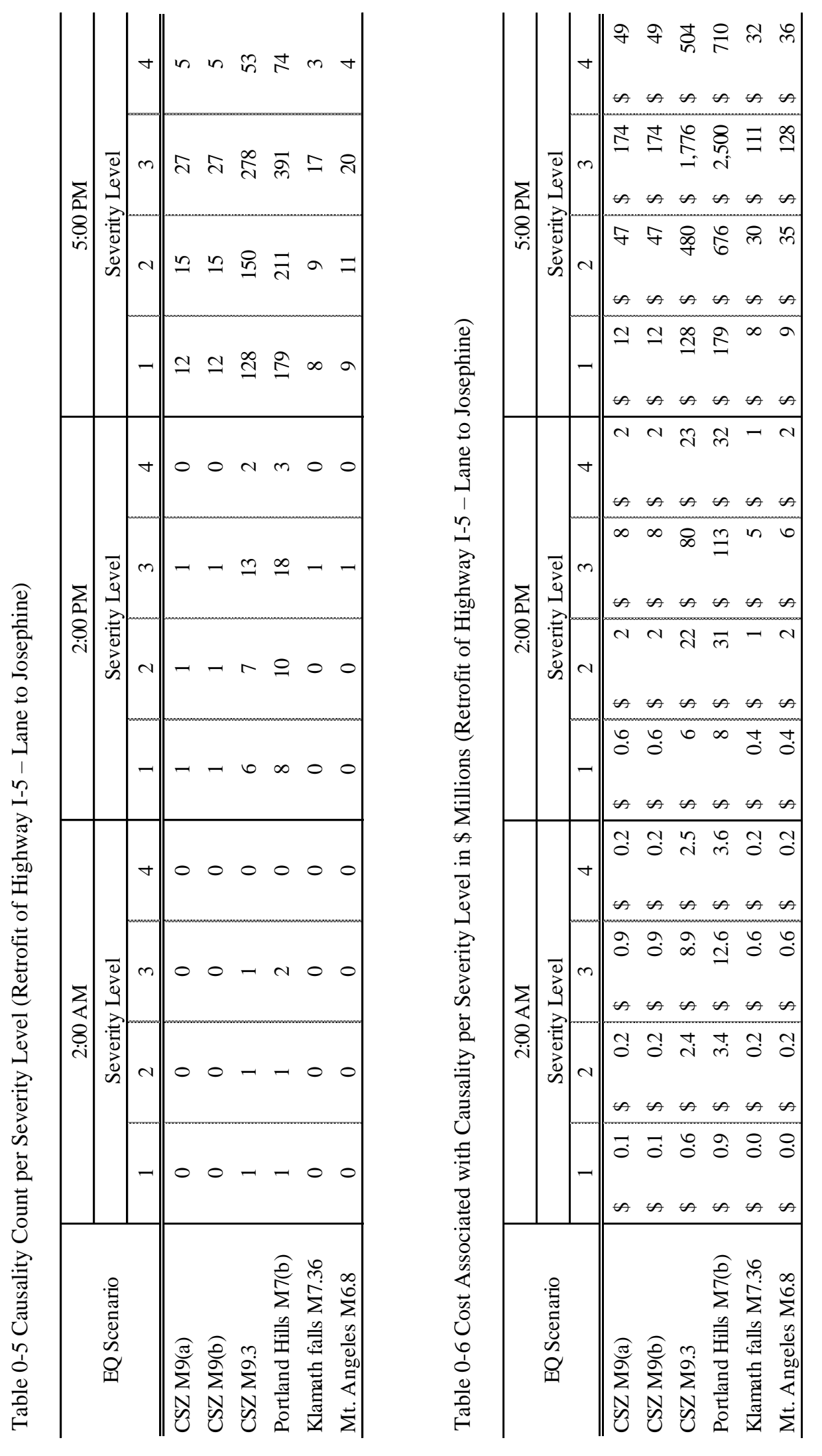

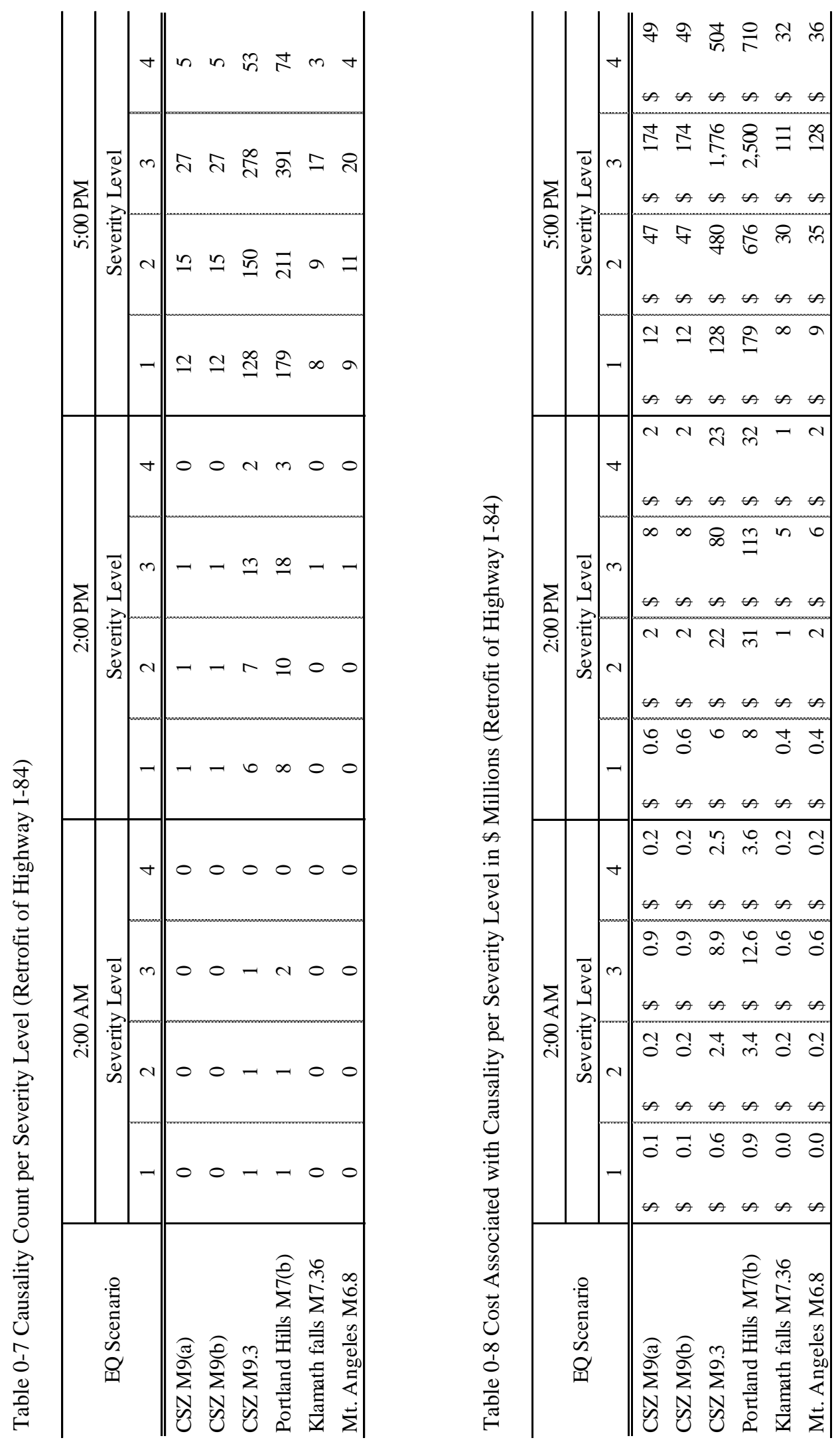

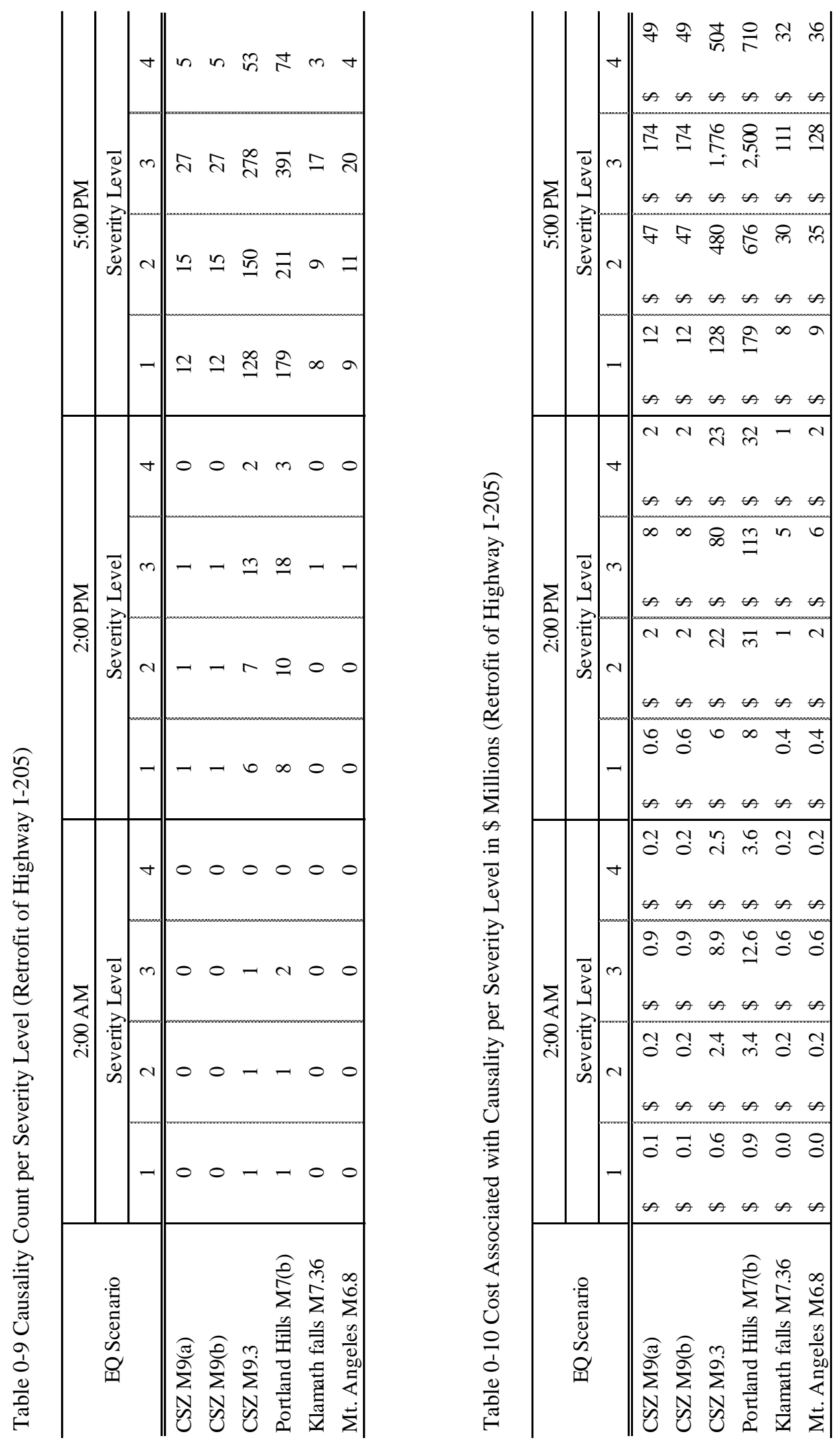

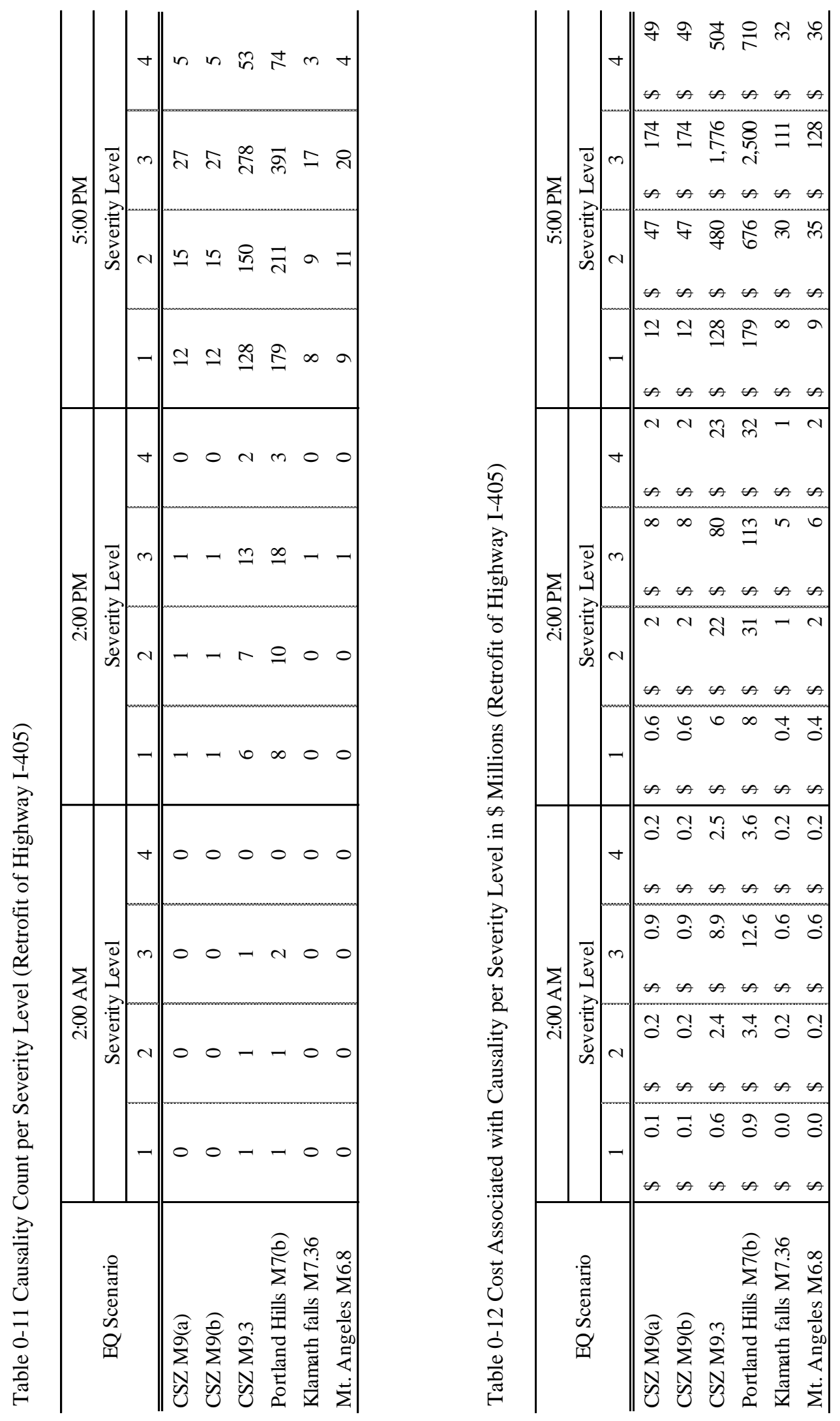

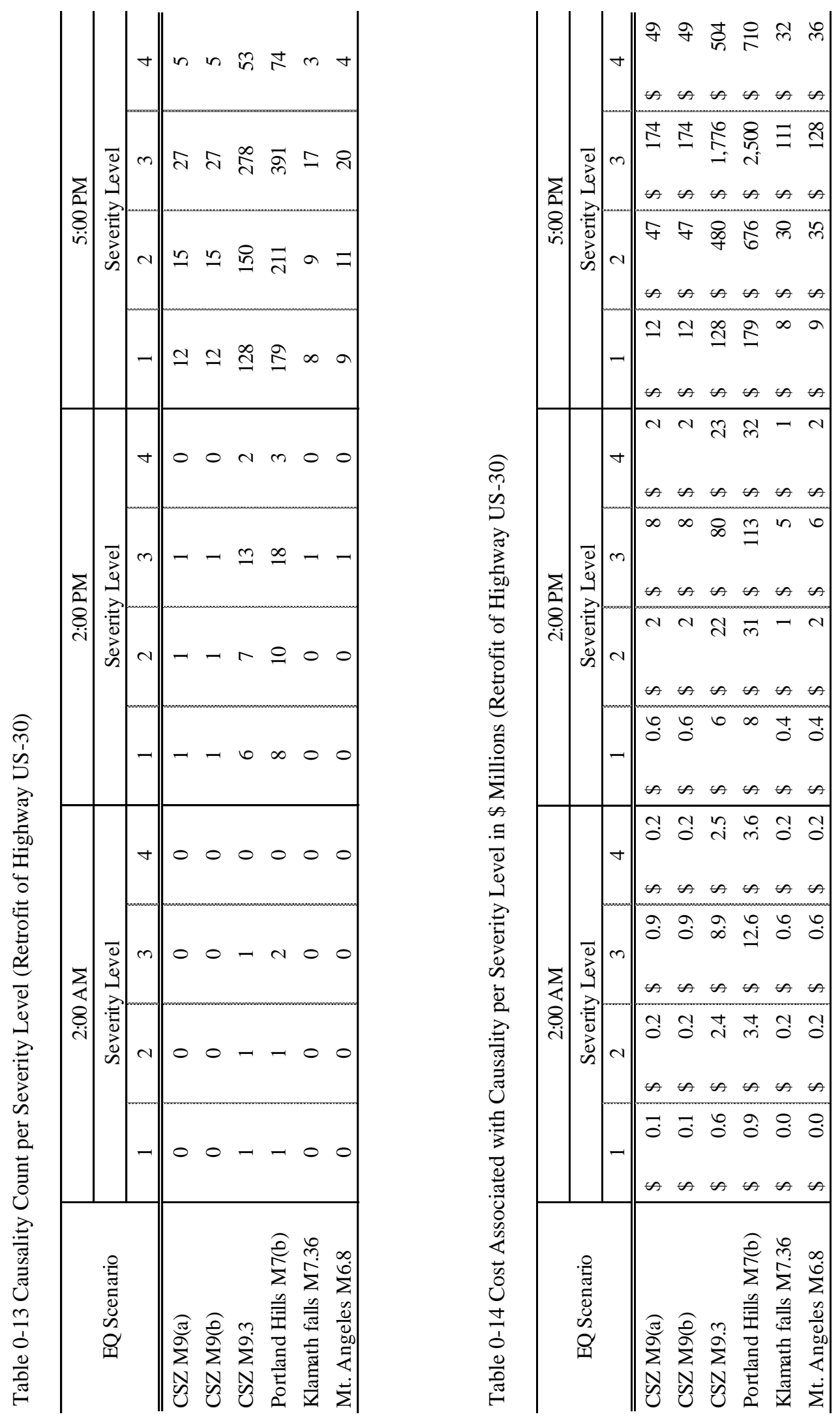

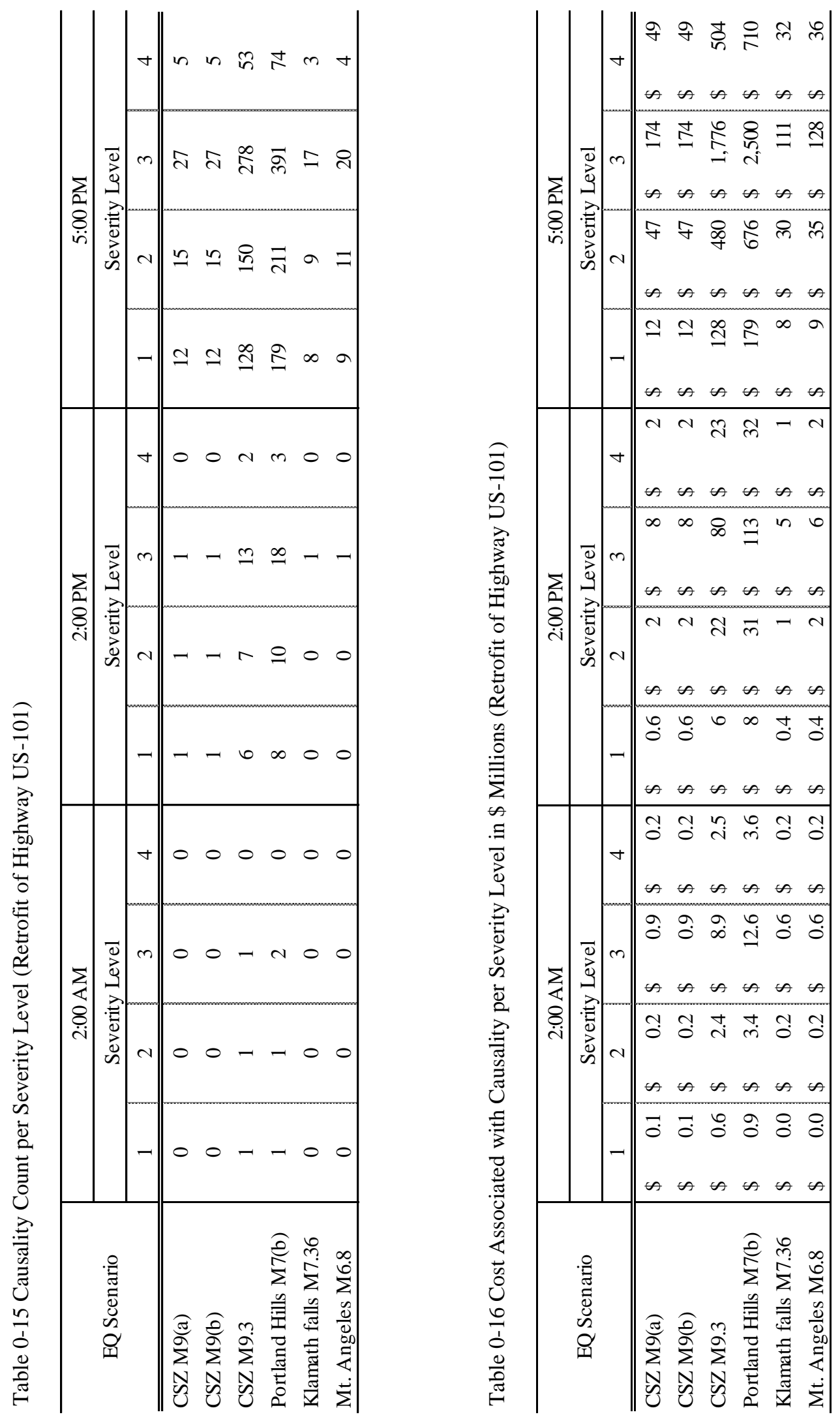

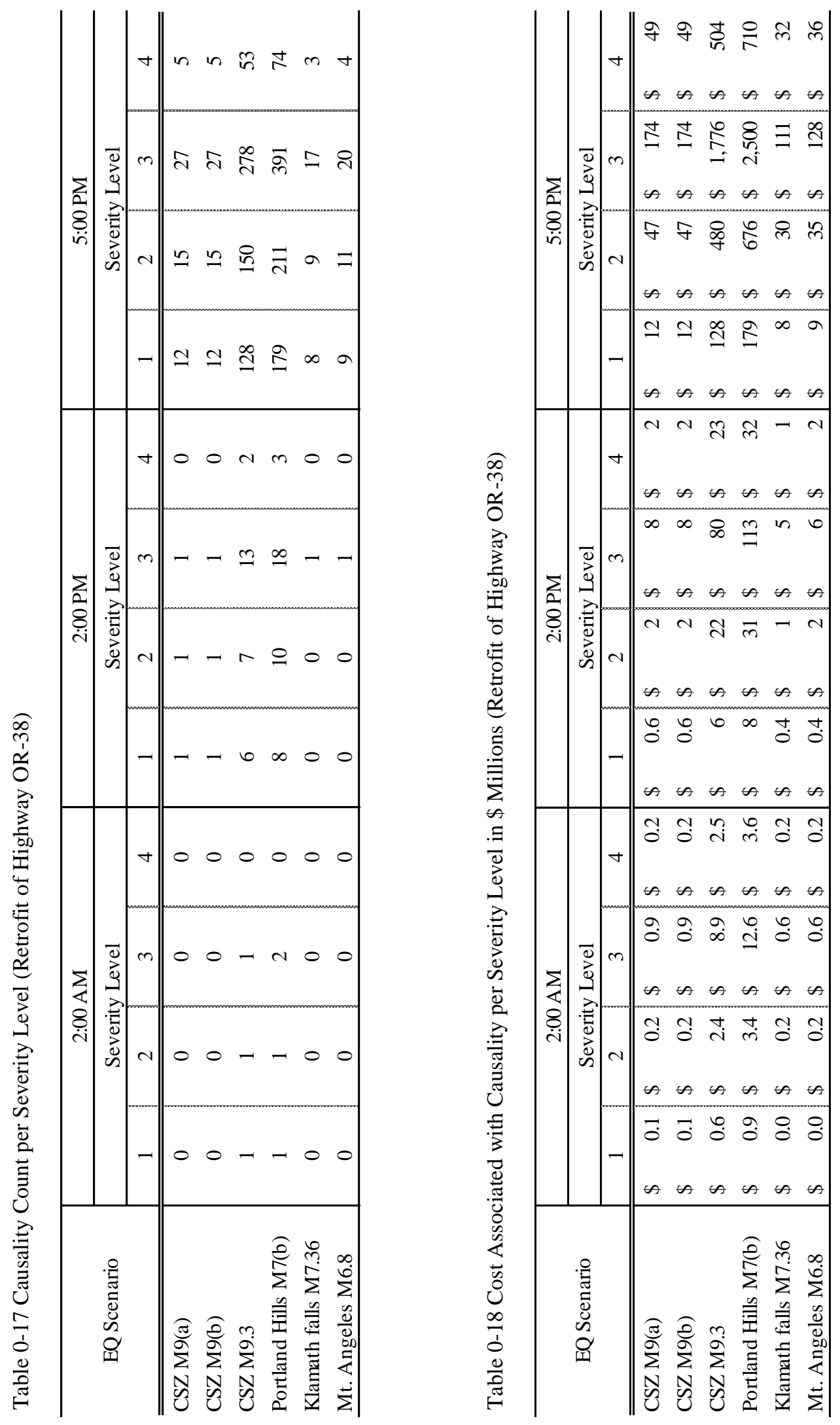

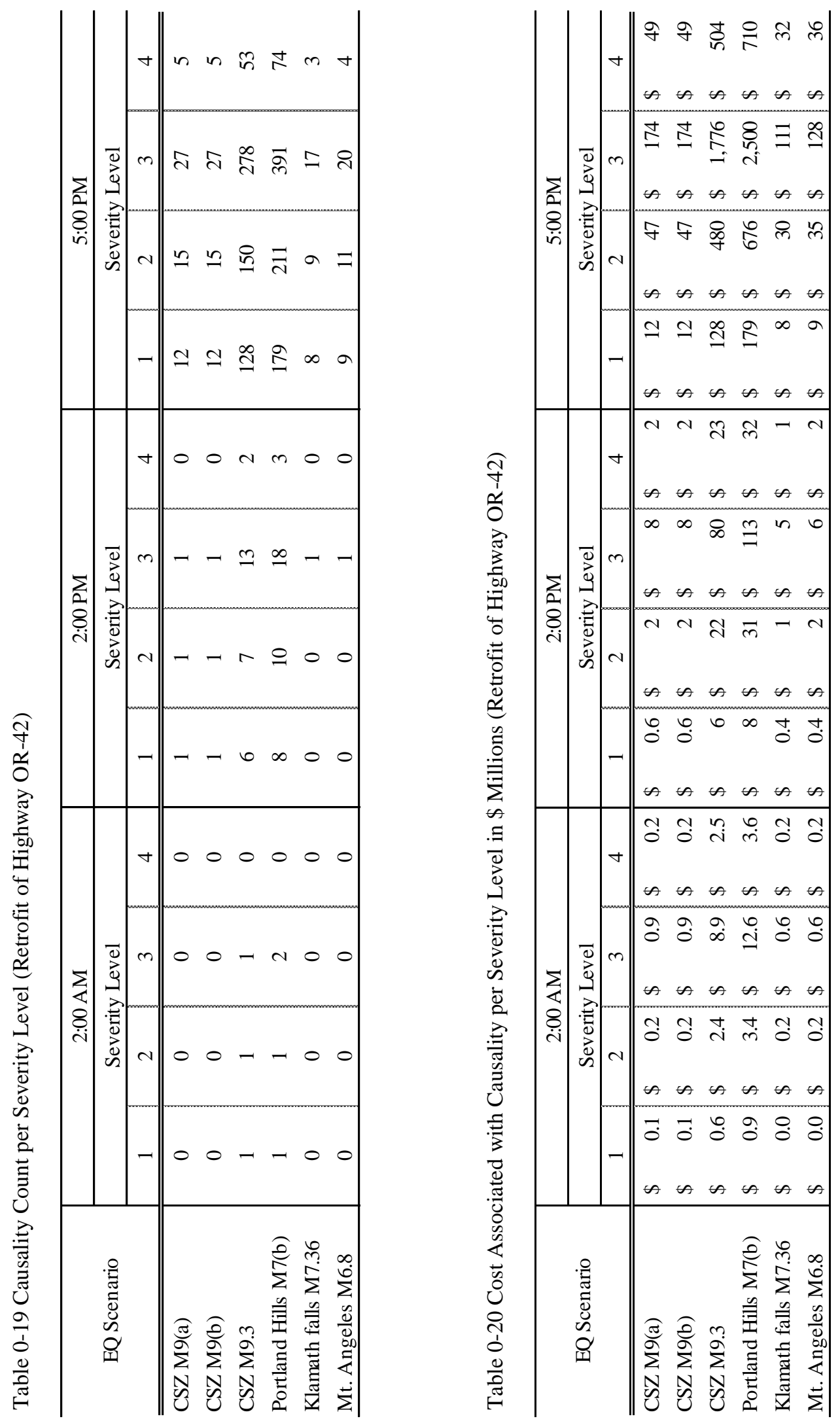

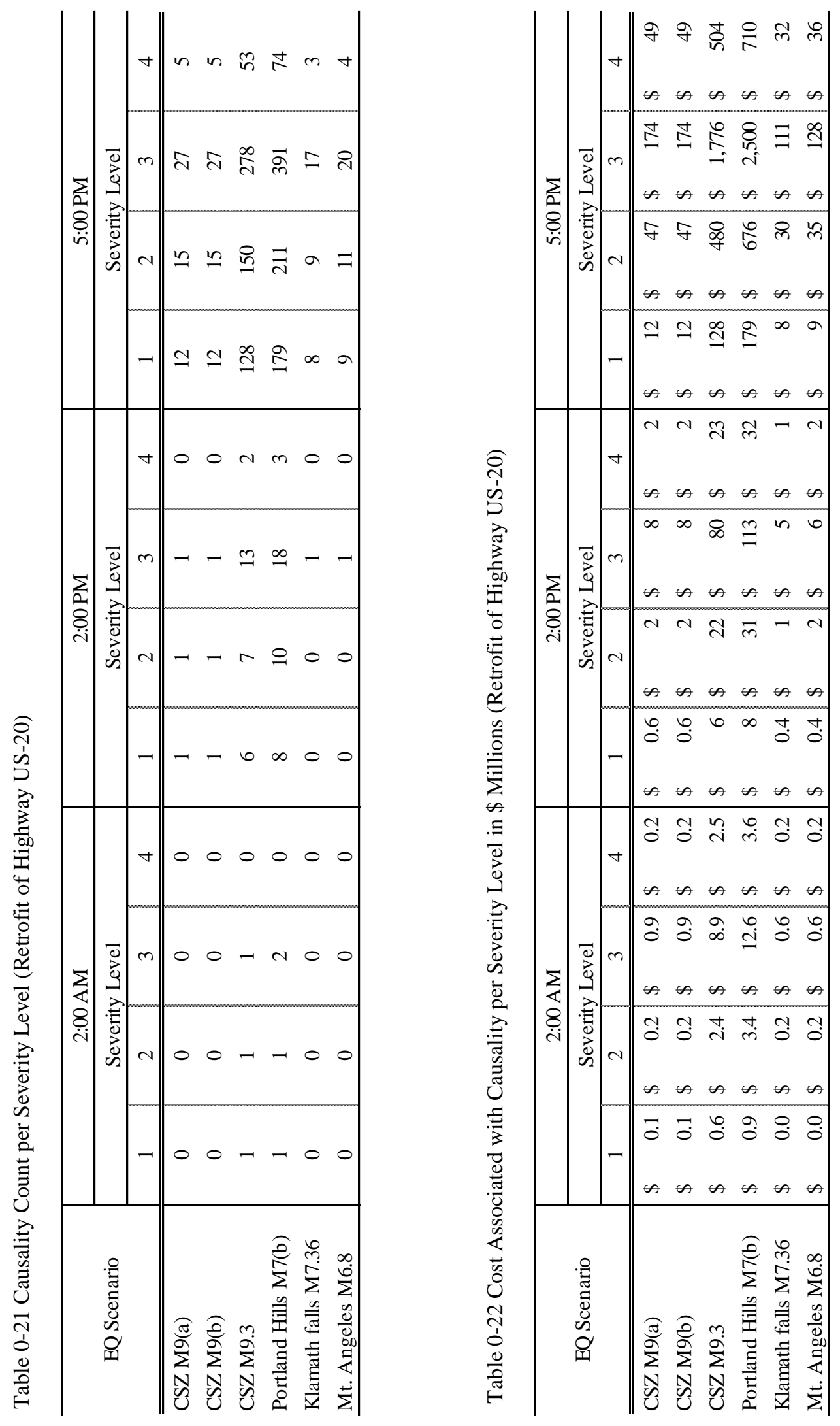

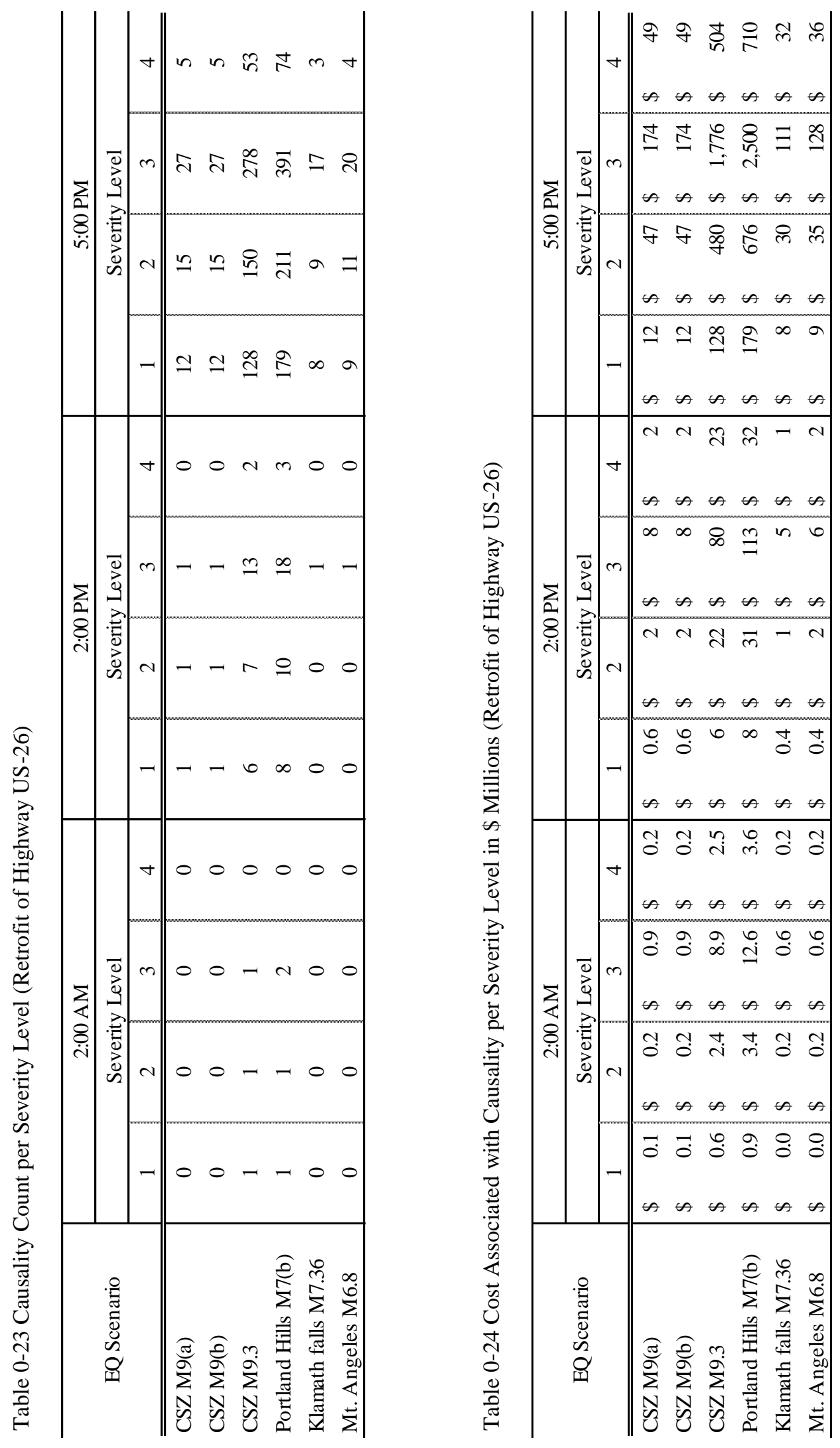